VILNIUS GEDIMINAS TECHNICAL UNIVERSITY

Tomas CUZANAUSKAS

\title{
INVESTIGATION OF MEDIA ACCESS CONTROL IN WIRELESS NETWORKS
}

DOCTORAL DISSERTATION

TECHNOLOGICAL SCIENCES,

ELECTRICAL AND ELECTRONIC ENGINEERING (01T)

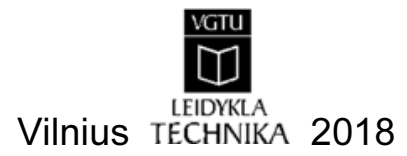


Doctoral dissertation was prepared at Vilnius Gediminas Technical University in 2013-2018.

\section{Supervisor}

Assoc. Prof. Dr Aurimas ANSKAITIS (Vilnius Gediminas Technical University, Electrical and Electronic Engineering - 01T) (2013-2016),

Prof. Dr Šarūnas PAULIKAS (Vilnius Gediminas Technical University, Electrical and Electronic Engineering - 01T) (2017-2018).

The Dissertation Defense Council of Scientific Field of Electrical and Electronic Engineering of Vilnius Gediminas Technical University:

\section{Chairman}

Prof. Dr Dalius NAVAKAUSKAS (Vilnius Gediminas Technical University, Electrical and Electronic Engineering - 01T).

\section{Members:}

Dr Rimvydas ALEKSIEJŪNAS (Vilnius University, Physics - 02P),

Prof. Dr Artūras SERACKIS (Vilnius Gediminas Technical University, Electrical and Electronic Engineering - 01T),

Dr Szymon SZOTT (Stanislaw Staszic Name Academy of Mining and Metallurgy, Electrical and Electronic Engineering - 01T),

Assoc. Prof. Dr Dainius UDRIS (Vilniaus Gediminas Technical University, Electrical and Electronic Engineering - 01T).

The dissertation will be defended at the public meeting of the Dissertation Defense Electrical and Electronic Engineering in the Senate Hall of Vilnius Gediminas Technical University at 2 p. m. on 27 August 2018.

Address: Saulètekio al. 11, LT-10223 Vilnius, Lithuania.

Tel.: +370 5274 4956; fax +370 5270 0112; e-mail: doktor@vgtu.lt

A notification on the intend defending of the dissertation was send on 26 July 2018.

A copy of the doctoral dissertation is available for review at VGTU repository http://dspace.vgtu.lt and at the Library of Vilnius Gediminas Technical University (Sauletekio al. 14, LT-10223 Vilnius, Lithuania) and the Wroblewski Library of the Lithuanian Academy of Sciences (Žygimantų st. 1, LT-01102, Vilnius, Lithuania).

VGTU leidyklos TECHNIKA 2018-035-M mokslo literatūros knyga

ISBN 978-609-476-123-2

(C) VGTU leidykla TECHNIKA, 2018

(C) Tomas Cuzanauskas, 2018

tomas.cuzanauskas@vgtu.lt 
VILNIAUS GEDIMINO TECHNIKOS UNIVERSITETAS

Tomas CUZANAUSKAS

\section{BELAIDŽIO RYŠIO TINKLỤ TERPĖS PRIEIGOS VALDYMO TYRIMAS}

DAKTARO DISERTACIJA

TECHNOLOGIJOS MOKSLAI,

ELEKTROS IR ELEKTRONIKOS INŽINERIJA (01T)

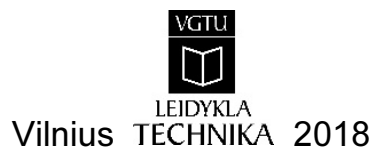


Disertacija rengta 2013-2018 metais Vilniaus Gedimino technikos universitete.

\section{Vadovas}

doc. dr. Aurimas ANSKAITIS (Vilniaus Gedimino technikos universitetas, elektros ir elektronikos inžinerija - 01T) (2013-2016),

prof. dr. Šarunas PAULIKAS (Vilniaus Gedimino technikos universitetas, elektros ir elektronikos inžinerija -01T) (2017-2018).

Vilniaus Gedimino technikos universiteto Elektros ir elektronikos inžinerijos mokslo krypties disertacijos gynimo taryba:

\section{Pirmininkas}

prof. dr. Dalius NAVAKAUSKAS (Vilniaus Gedimino technikos universitetas, elektros ir elektronikos inžinerija - 01T).

\section{Nariai:}

dr. Rimvydas ALEKSIEJŪNAS (Vilniaus universitetas, fizika - 02P), prof. dr. Artūras SERACKIS (Vilniaus Gedimino technikos universitetas, elektros ir elektronikos inžinerija - 01T),

dr. Szymon SZOTT (Stanislavo Stašico vardo kalnakasybos ir metalurgijos akademija, elektros ir elektronikos inžinerija-01T),

doc. dr. Dainius UDRIS (Vilniaus Gedimino technikos universitetas, technologijos mokslai, elektros ir elektronikos inžinerija - 01T).

Disertacija bus ginama viešame Elektros ir elektronikos inžinerijos mokslo krypties disertacijos gynimo tarybos posėdyje 2018 m. rugpjūčio 27 d. 14 val. Vilniaus Gedimino technikos universiteto senato posėdžių salejje.

Adresas: Saulètekio al. 11, LT-10223 Vilnius, Lietuva.

Tel.: (8 5) 274 4956; faksas (8 5) 270 0112; el. paštas doktor@vgtu.lt

Pranešimai apie numatomą ginti disertaciją išsiųsti $2018 \mathrm{~m}$. liepos $26 \mathrm{~d}$.

Disertaciją galima peržiūrèti VGTU talpykloje http://dspace.vgtu.lt ir Vilniaus Gedimino technikos universiteto bibliotekoje (Sauletekio al. 14, LT-10223 Vilnius, Lietuva) bei Lietuvos mokslų akademijos Vrublevskiu bibliotekoje (Žygimantų g. 1, LT-01102 Vilnius, Lietuva). 


\section{Abstract}

Over the years, consumer requirements for Quality of Service (QoS) has been growing exponentially. Recently, the ratification process of newly IEEE $802.11 \mathrm{ad}$ amendment to IEEE 802.11 was finished. The IEEE 802.11 ad is the newly consumer wireless communication approach, which will gain high spot on the $5 \mathrm{G}$ evolution. Major players in wireless market, such as Qualcomm already are integrating solutions from unlicensed band, like IEEE 802.11ac, IEEE 802.11ad into their architecture of LTE PRO (the next evolutionary step for 5G networking) (Qualcomm 2013; Parker et al. 2015). As the demand is growing both in enterprise wireless networking and home consumer markets. Consumers started to notice the performance degradation due to overcrowded unlicensed bands. The unlicensed bands such as $2.4 \mathrm{GHz}, 5 \mathrm{GHz}$ are widely used for up-to-date IEEE $802.11 \mathrm{n} / \mathrm{ac}$ technologies with upcoming IEEE 802.11ax. However, over usage of the available frequency leads to severe interference issue and consequences in total system performance degradation, currently existing wireless medium access method can't sustain the increasing interference and thus wireless needs a new methods of wireless medium access. The main focal point of this dissertation is to improve wireless performance in dense wireless networks. In dissertation both the conceptual and multi-band wireless medium access methods are considered both from theoretical point of view and experimental usage.

The introduction chapter presents the investigated problem and it's objects of research as well as importance of dissertation and it's scientific novelty in the unlicensed wireless field.

Chapter 1 revises used literature. Existing and up-to-date state-of-the-art solution are reviewed, evaluated and key point advantages and disadvantages are analyzed. Conclusions are drawn at the end of the chapter.

Chapter 2 describes theoretical analysis of wireless medium access protocols and the new wireless medium access method. During analysis theoretical simulations are performed. Conclusions are drawn at the end of the chapter.

Chapter 3 is focused on the experimental components evaluation for multiband system, which would be in line with theoretical concept investigations. The experimental results, showed that components of multi-band system can gain significant performance increase when compared to the existing IEEE 802.11n/ac wireless systems.

General conclusions are drawn after analysis of measurement results. 


\section{Reziumè}

Wi-Fi technologija tapo viena populiariausių dèl savo lankstumo ir pritaikymo galimybių. Paskutinis Wi-Fi standartas atvèrè dar didesnes galimybes integracijai 5G tinkluose. Naujas IEEE 802.11ad standartas nurodo naujas integracijos galimybes siekiant sukurti spartų 5G tinklą. Vienas didžiausių gamintojų „Qualcomm" numato šio standarto integraciją 4G tinkluose siekiant padidinti duomenu perdavimo spartą. Augantis įrenginių skaičius ir nekontroliuojama radijo prieigos terpè, nebeužtikrina patikimo duomenu perdavimo šiuolaikiniuose IEEE 802.11 tinkluose. Tai ypač pasireiškia $5 \mathrm{GHz}$ ir $2,4 \mathrm{GHz}$ dažnių juostose. $5 \mathrm{GHz}$ ir $2,4 \mathrm{GHz}$ dažnių juostos dèl augančio įrenginių skaičiaus tapo perkrautos, kas lèmè tolimesnį duomenų perdavimo spartos mažejimą.

Disertacijoje nagrinejamas Wi-Fi protokolas $2,4 \mathrm{GHz}, 5 \mathrm{GHz}$ ir $60 \mathrm{GHz}$ dažnių juostose. Pagrindinis dèmesys skiriamas sistemos našumo problematikai nagrinèti sutankintuose Wi-Fi tinkluose. Šiam tikslui pasiekti nagrinèjamos vieningos sistemos apjungimo galimybès. Disertacijoje nustatytos pagrindinès Wi-Fi bevielès prieigos problemos - tai neefektyvi laiko paskirstymo sistema, kuri nèra pritaikyta veikti, kai aptarnaujamas didelis vartotojų skaičius.

Ivadiniame skyriuje pristatoma disertacijos problema, tyrimo tikslai bei mokslinis naujumas. Aprašoma tyrimo metodologija ir ịvardijami ginamieji teiginiai.

Pirmajame skyriuje analizuojama dabartinès Wi-Fi sistemos kanalo prieigos schema, aptariami rinkoje esančiu ịrenginiu parametrai. Nagrinejjant Wi-Fi protokolo galimybes buvo atlikta mokslinių tyrimų analizè.

Ivardintas naujas MAC veikimo modelis Wi-Fi ịrenginiuose, kurio efektyvumas ištirtas bei ịvardinti komponentai, kurie sudaro bendrą protokolo visumą.

Antrajame skyriuje atlikta protokolo teorinè analizè, išnagrinètas eterio paskirstymo protokolas, atliktos lyginamosios analizès su kitais Wi-Fi MAC lygmens protokolais bei analizuoti IEEE 802.11 standarte nurodyti protokolai ir jų veikimas. Tyrimo metu apibrèžtas naujas radijo kanalo paskirstymo algoritmas. Atlikti teoriniai modeliavimai komponentų sudarančių bendrą Wi-Fi sistemą.

Trečiajame skyriuje atlikti eksperimentiniai palyginamieji matavimai su dabar taikomais Wi-Fi įrangos sprendimais. Aptariama kelių dažnių sistema bei pateikiami eksperimentiniai matavimų rezultatai.

Ivertinus matavimo rezultatus suformuotos bendros išvados. 


\section{Symbols}

$B$ - channel bandwidth;

$b_{i}$ - best response of player $i$;

$c_{i}$ - player's $i$ energy cost;

$h_{i i}$ - direct gain;

$h_{j i}$ - channel gain;

$N$ - players;

$n_{0}$ - noise power;

$P$ - power allocation profile;

$p_{i}-$ transmit powers of player $i$;

$p_{j}-$ tra nsmit powers of player $j$;

$u_{i}-$ global utility function.

\section{Abbrevations}

A-DCF - Advanced DCF;

AIFS - Arbitration Inter-Frame Spacing;

AP - Access Point;

ATIM - Announcement Traffic Indication Message; 
BC - Back-off Counter;

BEB - Binary Exponential Back-off;

BI - Beacon Interval;

BSS - Basic Service Set;

CAP - Controlled Access Phase;

CBAP - Contention Based Access Period;

CCA - Clear Channel Assessment;

CFP - Contention Free Period;

CoAP - Constrained Application Protocol;

$\mathrm{CP}$ - Contention Period;

CPE - Customer's Premise Equipment;

CQ - Control Queue;

CSMA/CA - Carrier Sensing Multiple Access/Collision Avoidance;

CTS - Clear to Send;

CWmax - Contention-Window Maximum;

CWmin - Contention-Window Minimum;

DCF - Distributed Contention Function;

DFS - Dynamic Frequency Selection;

DIFS - DCF Inter-frame Space;

DMG STA - Multi-Gigabit Station;

DTIM - Delivery Traffic Indication Map;

EDCA - Enhanced Distributed Channel Access;

EIRP - Effective Isotropic Radiated Power;

FST - Fast Session Transfer;

GI - Guard Interval;

GRAP - Group Randomly Addressed Polling;

GT - Game Theory;

$\mathrm{HC}$ - Hybrid Coordinator;

HCCA - HCF Controlled Channel Access;

HCF - Hybrid Coordination Function;

HT-LTF1 - High Throughput Long Signal Field;

HT-MF - High Throughput Mixed Mode Format;

HT-SIG1 - High Throughput Signal Field;

HT-STF - High Throughput Short Signal Field;

L-LTF - Legacy Long Training Field;

L-SIG - Legacy Signalling Field;

L-STF - Legacy Short Training Field;

MAC - Media Access Control Layer;

MAQ - MAC Queue;

MCS - Modulation Coding Scheme;

MP - Multi-Poll;

MPDU - MAC Protocol Data Unit;

viii 
NAV - Network Allocation Vector;

O-DCF - Optimized Distributed contention function;

PCF - Point Coordination Function;

PER - Packet Error Rate;

PHY - Physical layer;

PPS - Packets per second;

PTDMA - Pseudo-time Division Multiple Access;

PtMP - Point-to-MultiPoint;

QO - Quasi-Omni;

QoE - Ouality of Experience;

QSTA - Quality Stations;

RR - Round-Robin;

RSSI - Received Signal Strength Indicator;

RTS - Request to Send;

RTT - Round-Trip-Time;

STA - Station;

SNR - Signal Noise Ratio;

Soft-TDMA - Software Time Division Multiple Access;

STDMA - Spatial Time Division Multiple Access Protocol;

TBTT - Target Beacon Transmission Time;

TCP - Transport Control Protocol;

TDMA - Time Division Multiple Access;

TID - Traffic ID;

TIM - Traffic Indication Map;

TPC - Transmit Power Control;

TxOp - Transmission Opportunity;

UDP - Universal Datagram Protocol;

VR - Virtual Reality;

Wi-Fi - Wireless Fidelity;

WISPs - Wireless Internet Service Providers;

WM - Wireless Medium;

WRR - Weighted Round-Robin;

WSN - Wireless Sensor Network. 



\section{Contents}

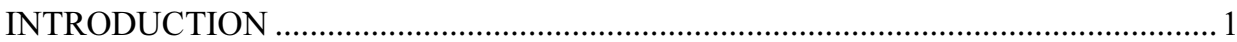

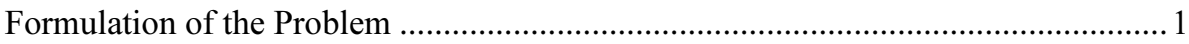

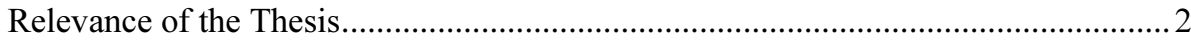

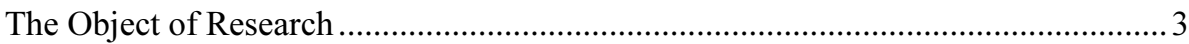

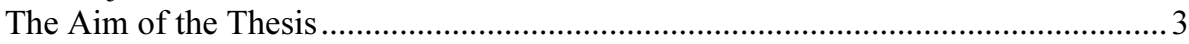

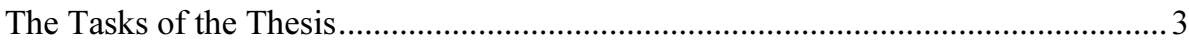

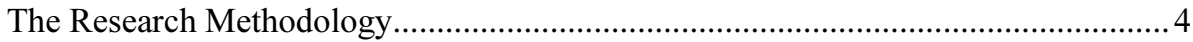

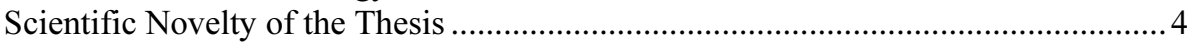

Practival Value of the Research Findings ...............................................................

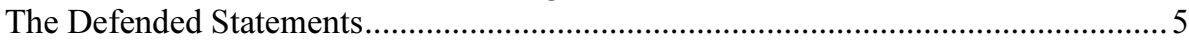

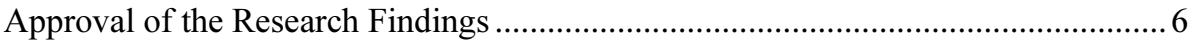

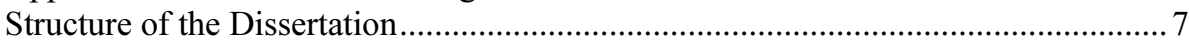

1. IEEE 802.11 CHALLENGES AND ADVANCEMENTS IN WIRELESS

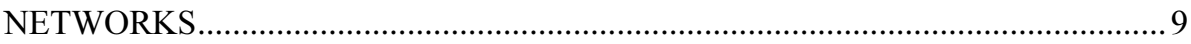

1.1. IEEE 802.11 Wireless Medium Access Control................................................. 9

1.2. IEEE 802.11 Advancements in Lithuania ...................................................... 12

1.3. Technological Advancements in IEEE 802.11 Media Access Control ............... 16

1.4. State-of-the-art Advancements in IEEE 802.11 Media Access Control.............22

1.5. IEEE 802.11 ad Standard $60 \mathrm{GHz}$ Advancements for Multi-band System .........26 
1.6. Power Control Algorithms in IEEE 802.11 Systems........................................26

1.7. Conclusions of Chapter 1 and Formulation of Thesis Tasks ................................. 35

2. THEORETICAL ANALYSIS OF MULTI-BAND WIRELESS SYSTEM ………......37

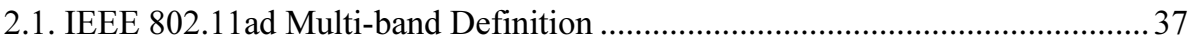

2.2. IEEE 802.11e Wireless Medium Access Control.................................................39

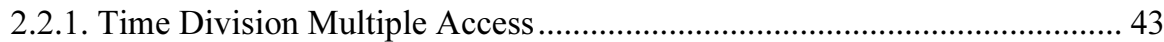

2.2.2. State-of-the-art Wireless Medium Access .................................................... 48

2.3. A Novel IEEE 802.11n Multi-Poll Protocol Implementation...............................54

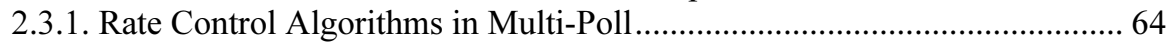

2.3.2. Game Theory based Power Allocation in Multi-Poll .................................... 68

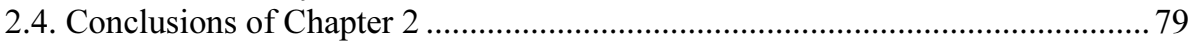

3. EXPERIMENTAL INVESTIGATION OF MULTI-BAND WIRELESS SYSTEM. 83

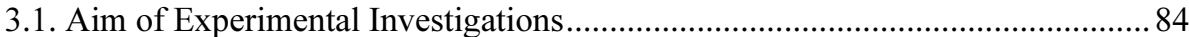

3.2. IEEE 802.11 Multi-Poll Experimental Investigation......................................... 84

3.2.1. The Short Distance Experimental Measurements.......................................... 88

3.2.2. The Hidden Node Scenario Experimental Measurements ............................. 95

3.2.3 The Round Trip Time Experimental Measurements ...................................... 98

3.2.4. Co-existence Between Legacy and Multi-Poll Protocol.................................. 99

3.2.5. Time Division Multiple Access and Multi-Poll Comparison ..................... 101

3.3. The Power Allocation in Multi-Poll Access Scheme ........................................ 104

3.4. Co-Channel Measurements in Outdoor Scenario .............................................. 107

3.5. Multi-band System Experimental Performance Measurements ....................... 108

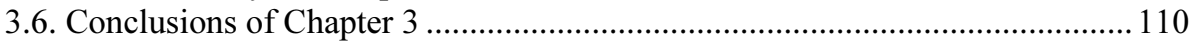

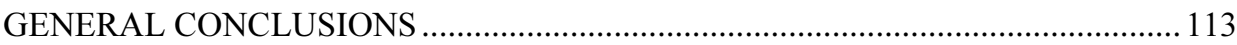

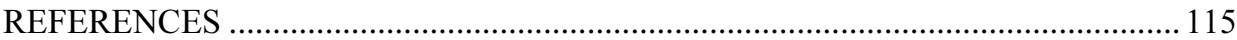

LIST OF SCIENTIFIC PUBLICATIONS BY THE AUTHOR ON THE TOPIC OF

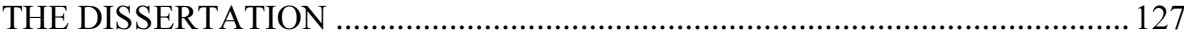

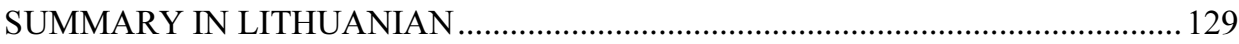

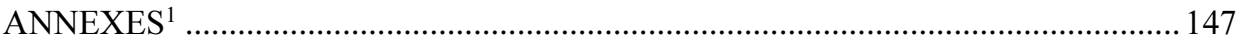

Annex A. Declaration of academic integrity ................................................... 148

Annex B. The coauthors agreements to present publications for the dissertation

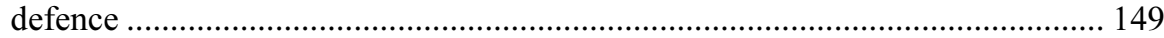

Annex C. Copies of scientific publications by the author on the topic of the dissertation

${ }^{1}$ The annexes are supplied in the enclosed compact disc

xii 


\section{Introduction}

\section{Formulation of the Problem}

The fast flourishing of Wi-Fi eco-systems was a consequence of simple regulatory rules and access to chunks of unlicensed spectrum band such as $2.4 \mathrm{GHz}$ or $5 \mathrm{GHz}$ ("Authorization of Spread Spectrum Systems Under Parts 15 and 90 of the FCC Rules and Regulations". Federal Communications Commission, USA, June 18, 1985). This lead to exponential grow of Wi-Fi popularity between Wireless Internet Service providers and offloading technology for licensed Long Term Evolution (LTE) or $3 \mathrm{G}$ networks (Report for the European Commission 2013). However, the growth itself without significant management rules over unlicensed spectrum lead to a high density and typically overcrowded wireless environment, where system performance started degrading to due increased noise levels (Hardin, Garrett 2007; Hanna, Sydor 2012; Lee et al. 2016, Zhang et al. 2016).

Existing wireless medium access mechanisms that are unchanged from the early IEEE 802.11 introduction, are not able to cope with increased interference over unlicensed bands and became outdated (Chatzimisios et al. 2004; Baid et al. 2015; Zhang et al. 2016). One of the factors is a scarce spectrum of unlicensed bands, since only limited amount of frequencies are available for usage. Another factor is the low spectral efficiency of Wi-Fi itself and thus quality is typically erratic in congested environments (Lee et al. 2016, Zhang et al. 2016). 
As IEEE 802.11 received several amendments: IEEE 802.11e, IEEE 802.11 ac and IEEE 802.11ad (IEEE 802.11 WG 2012) there is a need for more efficient spectral usage and lighter rules over power constrains in order to increase performance and propagation characteristics. However, this can be done only if the equipment would use smart techniques to increase fairness between wireless devices. Such techniques include smart transmit power control and rate adaption according to environment changes taking into account the operational basic service set area.

Therefore, the problem of inefficient and purely managed wireless medium access in IEEE 802.11 networks is addressed in the thesis.

In order to solve the defined problem, a hypothesis is raised and proven: an efficient Multi-Poll based wireless medium access scheme provides a beneficial and effective way to operate in high density wireless networks, which increases system capacity in comparison with IEEE 802.11 EDCA and IEEE 802.11 PCF (polling based) systems.

\section{Relevance of the Thesis}

Some of the most pronounced performance limitations with IEEE 802.11 WLAN devices is due to their Out of Band emission spectrum. Consequently, co-located Wi-Fi networks operating on adjacent channels see combined performance degradation that decreases only when there is increased physical separation between networks (Nachtigall et al. 2008; Doering et al. 2013; Hills 2001). Such problems give rise to poor spectrum efficiency and spectrum re-use, and contribute to the poor performance often noted within the unlicensed bands.

To overcome some of the Wi-Fi bandwidth limitations due to Adjacent Channel Interference Ratio, the IEEE 802.11 standards process implemented channel bonding functionality. Though in theory channel bonding significantly increases spectrum efficiency and utilization, practical deployments seem to indicate otherwise, especially in noisy environment (Deek et al. 2011; Shrivastava et al. 2008; Texas Instrument 2003, Fiehe et al. 2010).

Since the very first days of IEEE 802.11, its operation was based on two key mechanisms for accessing radio channel, namely the DCF (Distributed Coordination Function) and PCF (Point Coordination Function). The DCF mechanism is the only one that has been certified by the Wi-Fi alliance and as of today, it is the access mode of choice implemented in all the Wi-Fi compatible state of the art devices. Currently most of the devices rely on IEEE 802.11e and Enhanced Distributed Channel Access (EDCA) mechanism, which additionally adds Quality of Service Support (QoS) for Wi-Fi systems. DCF or EDCA works by employing mechanism called Carrier Sensing Multiple Access/Collision Avoidance 
(CSMA/CA). This mechanism exhibits poor performance in highly interfered environments, especially in high density of wireless devices where CSMA/CA decreases the overall performance due to inability to handle multiple collisions. This is related to both ineffective wireless medium sharing as well as rate control algorithm inability to distinguish between collision and channel fading.

Over the time that the Wi-Fi standard has been in place, many attempts have been made to improve the CSMA/CA protocol (Chatzimisios et al. 2004; Tinnirello et al. 2009). However, these changes, such as implementing RTS/CTS mechanism (Bruno et al. 2002), do not offer complete solutions (Jardosh et al. 2005; Chandra et al. 2008, Gast et al. 2013). Performance gains are highly dependent on the radio environment and operating in congested environments causes considerable throughput degradation (Pediaditaki et al. 2012).

In order to solve the problem, a such hypothesis is raised and proven - a higher efficiency Media Access Control (MAC) modification, that implements Multi-Poll capability proposes an efficient way to solve performance degradation problem with high number of connected stations in outdoor wireless network in comparison with IEEE 802.11 EDCA and IEEE 802.11 PCF based systems.

\section{The Object of Research}

The object of research is a IEEE 802.11 Medium Access Control layer improvement for building a more efficient wireless medium access technique, which would result in more effective Multi-band capable system.

\section{The Aim of the Thesis}

The aim of this work is to investigate and propose a efficient IEEE 802.11 Medium Access Control layer modification to increase wireless system's performance in dense wireless networks.

\section{The Tasks of the Thesis}

In order to achieve the goal of the thesis, the following tasks have to be solved:

1. To perform detailed theoretical and practical analysis of IEEE 802.11 Media Access Control Protocol.

2. To propose a IEEE 802.11 MAC layer changes, which would increase performance in outdoor based point to multipoint IEEE 802.11 networks. 
3. To perform comparative analysis against up-to-date existing IEEE 802.11 standards.

4. To implement a modification for multi-band system in order to increase existing IEEE 802.11 ad system efficiency by using multiple unlicensed bands with active client steering capability for FST function.

\section{Research Methodology}

To investigate the object, the following research methods are chosen:

- Classification: summarizing strength, weaknesses and gaps of literature, the dissertation research object should be recognized and understood.

- Hypothetical: theoretical study using existing IEEE 802.11 code base

- Experimental: the hypothesis should be tested by taking a practical test under different testing scenarios and location of the devices as well as using artificial network simulation utilities

- Statistical: analysis of 802.11 MAC layer performance using Python programming modelling utility

\section{Scientific Novelty of the Thesis}

The theoretical and experimental results have brought the following novel scientific novelty:

1. Created a novel MAC layer changes for IEEE 802.11 MAC, which increases system performance in point-to-multipoint wireless network topology.

2. The new theoretical and practical experiments introduces Game Theory as a mechanism for power allocation in greedy network topologies, where a high density scenarios are being deployed. The same methods can be applied for different set of devices, which operates in licensed or unlicensed band in distributed manner.

3. The new data of experimental measurement in outdoor based environment with hidden wireless nodes.

4. The new data of IEEE 802.11 TDMA techniques in existing of-theshelf devices as well as new measurements results. 
5. The benefit of the new Fast Session Transfer (FST) technique is evaluated in order to increase multi-band system's performance and perform active client steering between multiple bands as in comparison with single band system.

\section{Practical Value of the Research Findings}

A provided solution of extensive IEEE 802.11 MAC layer modification and expanding currently existing IEEE 802.11 operational state, increases performance in high density scenarios as well as provides better connectivity for rural areas by exposing such unlicensed spectrum in comparison with existing IEEE 802.11 EDCA and IEEE 802.11 PCF based implementations.

Finally, IEEE 802.11 ad is considered as next technology for both indoor and outdoor usage. Therefore, building a multi-band capable system with proposed extensive FST functionality, would allow to use $5 \mathrm{GHz}$ and $60 \mathrm{GHz}$ bands more efficiently, thus increasing system's capacity.

Through the thesis, several recommendations and important IEEE 802.11 MAC layer wireless medium access improvements increases overall IEEE 802.11 system performance. The suggestions and practical implementations are already used on commercially deployed devices by WISPs.

Additionally, the achieved results are used in Joint Polish-Lithuanian Daina project proposal. The proposed project builds a new theoretical and practical background set of ideas based on the achieved results in this dissertation.

\section{The Defended Statements}

1. The novel Multi-polling based MAC layer implementation increases data throughput up to $12 \%$ for 13 active clients setup in comparison with PCF (polling based without Contention Period) data throughput and up to $79 \%$ in comparison with EDCA data throughput. For 60 active clients setup, Multi-Poll increases data throughput up to $50 \%$ in comparison with PCF (polling based without Contention Period) and up to $388 \%$ in comparison with EDCA, when TCP bidirectional streams are being generated over clients.

2. The Multi-polling based MAC layer implementation increases data throughput up to $89 \%$ in comparison with TDMA implementation, that uses variable timeslot for data transfer and has only one active client and bidirectional TCP traffic is being sent. 
3. The modification of IEEE 802.11 MAC layer with active and idling client scheduling provides up to 12 times better data packets latency in comparison with IEEE 802.11 PCF (polling based without Contention Period) based approach when setup consists of more than 60 clients and one of the clients is sending bidirectional data.

4. $60 \mathrm{GHz}$ IEEE 802.11ad wireless system can expose FST approach as leverage to steer the low rate or idle client to $5 \mathrm{GHz}$ band, that increases $60 \mathrm{GHz}$ data throughput up to $18 \%$ in comparison with $60 \mathrm{GHz}$ PtMP setup only, when all connected clients are actively sending data.

\section{Approval of the Research Findings}

Research results on the dissertation subject are published in 12 scientific articles: 1 article - Clarivate Analytics Web of Science with impact factor, 3 in other databases, 8 - Clarivate Analytics Web of Science data base "Conference Proceedings". Eight presentations have been made in international conferences:

- International scientific conference "Advances in Information, Electronic and Electrical Engineering 2013”. 2013, Riga, Latvia.

- International scientific conference "IEEE International symposium on dynamic spectrum access networks 2014”. 2014. McLean, Washington, USA.

- International scientific conference "9th International conference on cognitive radio oriented wireless networks and communications 2014". 2014, Oulu, Finland.

- International scientific conference "Advances in Information, Electronic and Electrical Engineering 2015”. 2015, Riga, Latvia.

- International scientific conference "Electrical, Electronic and Information Sciences 2015”. 2015, Vilnius, Lithuania.

- International scientific conference "11th IEEE International Wireless Communications and Mobile Computing Conference 2015”. 2015. Dubrovnik, Croatia.

- International scientific conference "Electronic and Information Sciences 2016”. 2016, Vilnius, Lithuania.

- International scientific conference "Electronics 2017”. 2017. Palanga, Lithuania. 


\section{Structure of the Dissertation}

The volume of the dissertation is 162 pages. Dissertation contains 51 figures, 9 tables, 10 numbered formulas, 152 references are used. 



\section{IEEE 802.11 Challenges and Advancements in Wireless Networks}

This Chapter reviews current Wi-Fi system trends and approaches on different scientific goals. To begin with, a different components of Wi-Fi system are investigated, that include wireless medium access schemes, packet aggregation techniques, rate control modules and power control options. Main focus of this chapter is to establish a background for wireless medium access scheme improvement.

The material presented in this chapter was published in (Cuzanauskas, Anskaitis 2016a; Cuzanauskas, Anskaitis 2016b; Cuzanauskas, Anskaitis 2016c; Cuzanauskas, Anskaitis 2015a) and presented in two international conferences: (Riga 2013), (Vilnius 2016).

\subsection{IEEE 802.11 Wireless Medium Access Control}

In the beginning, IEEE 802.11 had two basic functionalities for wireless medium access. The first one, that is mainly used even today, is called Distributed Coordination Function (DCF) (IEEE Std 802.11-2016). The DCF is an algorithm, that operates as a function listen before sending any data. Every wireless device tries 
to send data only if the wireless medium is not busy. This is a good option for indoor applications where the most of the wireless nodes are located in the same space or so called Basic Service Set (BSS) area. Differently than DCF, Point Coordination Function (PCF) is based on central entity usually Access Point (AP). The PCF uses a Contention Free Period (CFP) for communication. AP constantly sends tokens to poll connected stations and allows them to transmit data in sequence. The polling frames or tokens, are used to control the wireless medium and resolve the hidden wireless node problem between wireless devices. However, the stations are polled even if they do not have data. This creates additional spectrum inefficiency, where a waste of time occurs for stations which do not have any data to transmit. The inefficiency is related to additional management frames transmission even without actual data being transmitted (Chou et al. 2013).

The update of IEEE 802.11 named IEEE 802.11e added QoS enhancements for wireless nodes. The IEEE 802.11e standard (Klein 2008) allowed supporting different levels of priorities for voice, video, best-effort and background data. The mechanism itself relies on parameters such as Arbitration Inter-Frame Spacing (AIFS), Transmission opportunity (TxOp), Contention-Window Minimum (CWmin), and Contention-Window Maximum (CWmax). These parameters are left for individual configuration and only recommended values are left as defaults for the most of the Wi-Fi based devices. However, these values might be suboptimal in different wireless conditions and requires dynamic adjustment based on wireless environment. The IEEE 802.11e specifies two wireless access schemes: HCF Controlled Channel Access (HCCA) and Enhanced Distributed Coordination Access (EDCA) (Fallah, Alnuweiri 2007).

The HCCA operates in similar way as former PCF, the difference in this case is the added QoS with four queues for different type of traffic. The access categories are separated by using different contention parameters, that creates a differentiation how queues can access the wireless medium (Perahia, Stacey 2013). EDCA is a former DCF, the same CSMA/CA operation is applied here and same as HCCA it has four queues for different type of traffic. The CSMA/CA mechanism which is common for 802.11 MAC is referred as Distributed Coordination Function (DCF). All stations which participate for wireless medium access performs Clear Channel Assessment (CCA). This is done by using both virtual carrier sense mechanism as well as physical (transmit power) energy detection mechanism. The period for which the CCA is performed is called DCF Inter-frame Space (DIFS). If the medium is idle for such period, stations can reserve channel and start transmissions. If during CCA period wireless medium is busy, stations wait for another DIFS plus a random back off period. Upon gaining wireless medium, station maintains the occupation of channel by using Short Inter-frame Space (SIFS) between data packets. Therefore, none of other stations can interrupt the 
transmission, since they are sensing channel for DIFS period which is longer compared to SIFS. However, 802.11 has transmission time rules, which prevents individual station from occupying the wireless medium too long.

CSMA/CA re-uses fundamental mechanisms of carrier sense. Energy based detector resides in Physical (PHY) layer and detects energy levels in channel as well as preambles with frame length.

In basic principle virtual carrier sense derives packet duration field of the wireless header and takes that duration as a period for which wireless medium will be busy (Perahia, Stacey 2013). The virtual carrier sense mechanism is known as Network Allocation Vector (NAV). The medium is considered as idle only when NAV and PHY carrier sense mechanisms indicates that wireless medium is idle. In order to further minimize possibility of collisions stations might use request to send (RTS) and clear to send (CTS) control frames, which are modulated with lowest Modulation Coding Scheme (MCS) and therefore can be seen by higher amount of stations. By receiving RTS, nearby stations set their NAV to value and initiates back off for the period which is set by extracting data from RTS frame. Additionally, the extracted data is used for future pattern predictions and is being constantly updated when valid Physical Layer Service Data Unit (PSDU) frame is received. However the NAV is not being updated if the received frame was meant to this particular station. Most of the current driver implementations do not use any traffic pattern predictions based on the NAV (IEEE Std 802.11-2016).

The reservation of NAV update procedure can be performed by using so called RTS/CTS mechanism. When RTS and CTS frames are being exchanged between station and AP. Some of stations might receive only CTS, since CTS is being transmitted by AP to all the stations that are in the AP's operation field, since typically it might happen that initial station, which sent RTS was not in the same proximity as the other stations and therefore RTS was not received by stations contending in the same wireless medium.

However, such approach has a lot of drawbacks, since high amount of RTS and CTS frame exchange occupies airtime (Kosek-Szott et al. 2017), this is because these control frames are being transmitted at lowest data rate $6 \mathrm{Mbps}$ which reserves wireless medium for long duration. Therefore, a typical wireless network tries to avoid extensive usage of RTS and CTS frame mechanism and only in cases when frame transmission fails for several times uses RTS and CTS exchange to reserve wireless medium. The RTS and CTS flags are being controlled by rate control modules in IEEE 802.11 wireless driver. The Fig. 1.1 shows the wireless medium access scheme. In this case there are two operational stations, one of the stations is receiving data from the AP. The inter-frame spacing time intervals are: SIFS, DIFS, Extended Interframe space (EIFS), PCF Interframe space (PIFS) and AIFS in EDCA wireless medium access scheme. The EIFS is being used to delay 
the transmission in DCF, when the frame which was received before had an incorrect checksum or contained an error.

Back-off time while medium is idle

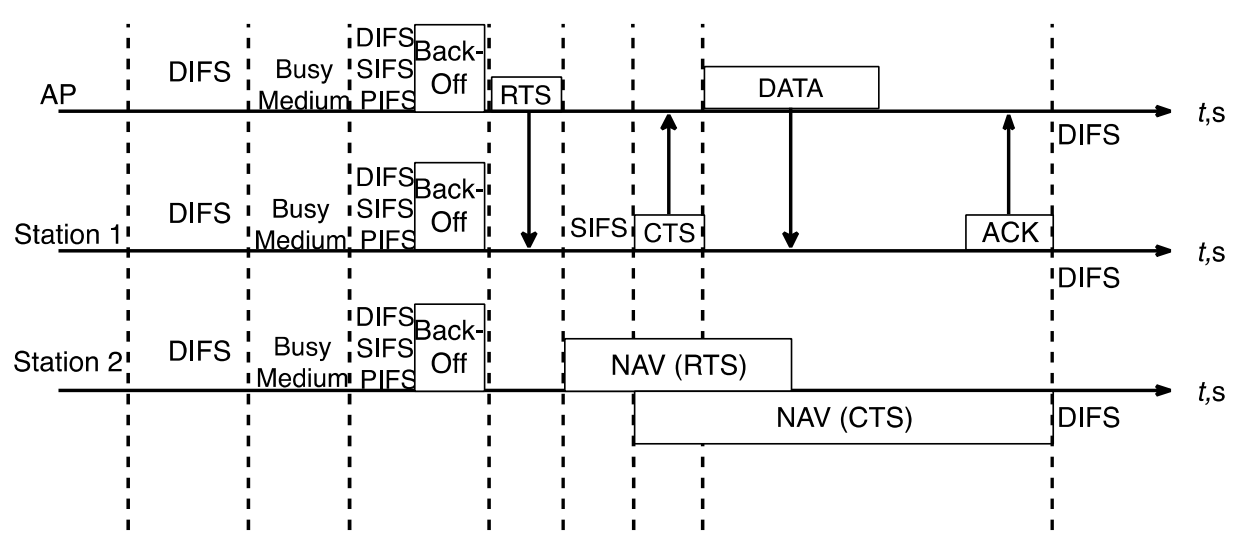

Fig. 1.1. The IEEE 802.11 Enhanced Distributed Channel Access scheme

The less common Reduced Interframe Space (RIFS), which are being used for transmission separation from single transmitter, but is being defined as obsolete since (IEEE Std 802.11-2016). The typical operation of EDCA as in Fig. 1.1. consists, that the station which wins the contention period is allowed to start the transmission. The remaining stations, in this case station 2 sets their NAV period for the transmission time required by the AP in $\mu$ s. The station 2 can start contention only after the NAV period ends and medium is clear during next time it attempts to listen.

In these days EDCA is the mainly used mechanism for wireless medium access, while HCF is rarely implement on any commercial based device due to its complexity. From QoS perspective the most of devices have fixed parameters that result in poor performance in the outdoor scenario, where more than one active clients are connected (Fallah, Alnuweiri 2007).

\subsection{IEEE 802.11 Advancements in Lithuania}

To begin with, advancements on IEEE 802.11 development in Lithuania can be separated into different directions:

1. Statistical evaluation of Wi-Fi Internet Access Networks.

2. Protocol evaluation under different use-cases. 
3. Networking performance improvement methods in IEEE 802.11 wireless networks.

The first research focus on data systemization, which is extracted by monitoring wireless networks over long period of time. The aim of such research is to provide insight of Wi-Fi operation under error prone environmental conditions, when saturation and channel occupation is high. This statistical information provides a possibility to make a proper network planning and channel selection under different operational areas (Japertas, Slanys 2013).

The second research starts by examining the IEEE 802.11 media access control layer (MAC) and provides a detailed and well covered experimental and theoretical analysis of IEEE 802.11 wireless standards (Saltis 2004; Pavilanskas 2007). The first thesis main research aspects consist of actual voice transmission over wireless network and packet aggregation mechanism, which was proposed (Saltis 2004). While the second thesis is taking a deeper look on IEEE 802.11 Technological Expenditures (overhead) which is added to the real data, while transmitting frames over the air. Some suggestions like overhead compression techniques are analyzed.

Additionally, authors consider voice application scenarios, which requires proper synchronization between voice and wireless protocol (Pavilanskas 2007). One of existing IEEE 802.11 protocols, Point Coordination Protocol, is suggested as proper solution to serving voice services to last mile users. Similar voice services transfer over wireless networks has been researched in another article (Pauliukas, Vosylius 2009; Matusevicius et al. 2015). The main approach of both these researches is to analyze how well the voice packets are transferred when using PCF based approach. The conclusion was that PCF is better suited for voice services as in comparison with DCF based approach, in cases when network load is high.

Furthermore, in many research papers a measurement of TCP packet loss is analyzed as well as different kinds of TCP protocols operation under error prone conditions, which is a common environmental factor while using wireless technology (Pavilanskas, Statkus 2010; Statkus, Paulikas 2013), which operates error prone conditions. However, while looking at these research directions, one can get a thought that nowadays wireless protocols are getting highly complex and last mile users, typically are not satisfied by one type of service (i.e. voice service). The trend nowadays is to propose triple play services like voice, video and standard data to last mile users (Bruzgiene et al. 2014). Nevertheless, concentration of actual devices, which are used by WISPs (Wireless Internet Service Provides), is getting dense and spectrum occupation on single tower is high. High investments are required to maintain the operational costs, which creates a new requirement such as aggregation of multiple users on single device to cut down the service 
costs, while still providing triple play services. Advanced techniques as co-channel filtering or frequency re-use became a standard method for currently fairly simply built IEEE 802.11 devices. Thus, more complex evaluation is required of IEEE 802.11 application to nowadays demands. Evaluation of security overhead is considered as well in dense wireless networks and is being analyzed in some of research papers (Dekeris, Narbutaite 2007).

The third research includes actual changes to the protocol usage and suggestion to better performance while using IEEE 802.11 based devices (Rindzevicius et al. 2006). These include analysis of Transport Control Protocol (TCP) behavior over wireless networks and suggestions to IEEE 802.11 where TCP Acknowledgement frames would be filtered or passes through without IEEE 802.11 Acknowledgement mechanism as simple as just send a single frame without checking if it's successfully received at the other end. This was researched on articles by (Pavilanskas, Statkus 2010; Statkus, Paulikas 2013). The idea is to filter out the TCP Acknowledgement frames, this is done based on theory that TCP protocol itself generates redundant ACK frames and only the newest matters, since it acknowledges all the frames sent before. Therefore, the old ACKs can be dropped. In order to verify such methodology authors used non-wireless devices and applied filtering rules based on classes, which allowed dropping outdated TCP ACKs. It was verified that even dropping up to $80 \%$ of TCP ACKs, there are no significant performance degradation on single TCP session stream. The aim of such techniques is to decrease the number of technological expenditures over IEEE 802.11 networks and achieve additional performance while eliminating unnecessary ACKs both at network layer and MAC layer of IEEE 802.11 protocol.

The main concerns for these papers is that such performance increase while removing TCP ACKs can be achieved over IEEE 802.11 which is still lacking the proper aggregation mechanism. The more up-to-date versions of 802.11 like IEEE 802.11 ac doesn't show the same performance degradation while sending multiple TCP ACKs. This can be explained by better aggregation schemes which are used in newer wireless protocol versions and therefore TCP filtering provides less gain in indoor application scenarios. However, TCP filtering techniques are still not researched in high density outdoors applications where users can see more than 70 operational clients on single Access Point. By implementing a redundant TCP Acknowledgement packet filtering mechanism and not relying solely on the link quality parameters to turn them off completely as in previous research attempt, the throughput gains in high density scenario became significant.

Due to dynamics of wireless networks, the Downlink and Uplink capacity might vary. This is called an asymmetrical link situation, where either Uplink or Downlink is limited due to interference or other situations. To deal with such cases, it is possible to implement TCP ACKs filtering technique which is mainly adopted in satellite communications and in other type of asymmetrical networks. 
The TCP ACKs flow guarantees growing throughput and TCP mechanism requires a steady and stable flow of ACKs. While usually, this is not a problem in wired communication in wireless networks there might be a case, where TCP ACKs are lost or outdated due to wireless medium occupation.

TCP accelerator mechanism is implemented both in stations and Access Point. The TCP ACKs are moved to different queue than all the data packets. When the stations receive control frame for polling, only the up-to-date ACKs are sent firstly from TCP ACKs queue. All the outdated or redundant ACKs, which acknowledge the same data frames, are dropped. When stations receive permission for transmission, firstly the TCP ACKs queue is sent and later a data queue. This allows handling jitter and latency, which is one of the important parameters for steady TCP flow.

During testing on IEEE 802.11ac devices, a network topology relied on standard CUBIC TCP implementation, which is common between Linux based systems. The CUBIC TCP provides one important capability and allows not to rely on TCP ACKs to increase the TCP Window size. This allows removing as many redundant TCP ACKs as possible, thus increasing the spectral efficiency.

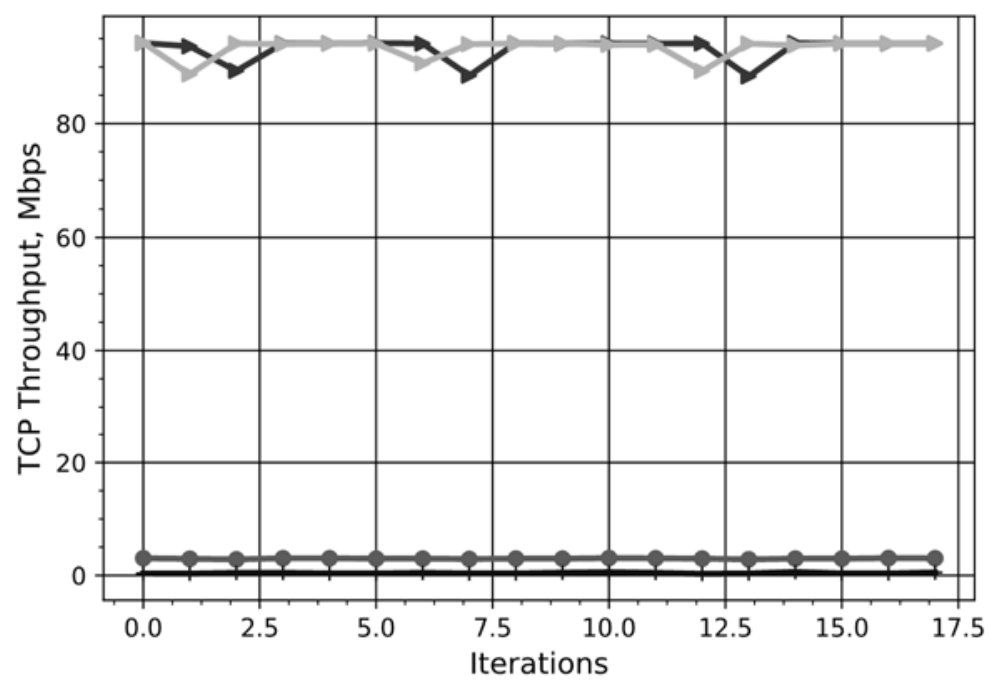

Stream of ACK (without TCP ACK filtering) Multi-Poll Downlink without filtered ACKs

- Stream of ACK (with TCP ACK filtering) $\rightarrow$ Multi-Poll Downlink with Filtered ACKs

Fig. 1.2. Transport Control Protocol Acknowledgements with and without active filtering 
In the first test, a throughput is generated just by TCP ACKs. Nepim tool was used to generate traffic only to one direction (simplex) and monitoring the generated traffic by TCP ACKs on the device. The results are displayed in Fig. 1.2, as it is noticed TCP ACKs created almost steady 2 Mbps traffic from stations to AP. When TCP filtering was enabled, the number of TCP ACKs decreases and less than $0.25 \mathrm{Mbps}$ is generated from station to AP.

This decreases redundant TCP ACKs and allows using wireless medium more efficiently. When running TCP ACK filtering the redundant ACKs removal decreases the ACK stream 8 times, which is especially useful in low processing power based systems or high density systems. This decrease of redundant traffic is beneficial for further Multi-band system build up.

\subsection{Technological Advancements in IEEE 802.11 Media Access Control}

Typically, 802.11 systems can be categorized based on protocol operation type: Enhanced Distributed Channel Access, which is a former distributed contention function with QoS capabilities, HCF Controlled Channel Access, which is a former point coordination function with QoS capabilities and Hybrid Channel Access methods.

However, different kind of media access control layer (MAC) functionality, can be applied in different locations and therefore wireless medium access schemes can be categorized based on use-cases, where different type of MAC changes are required.

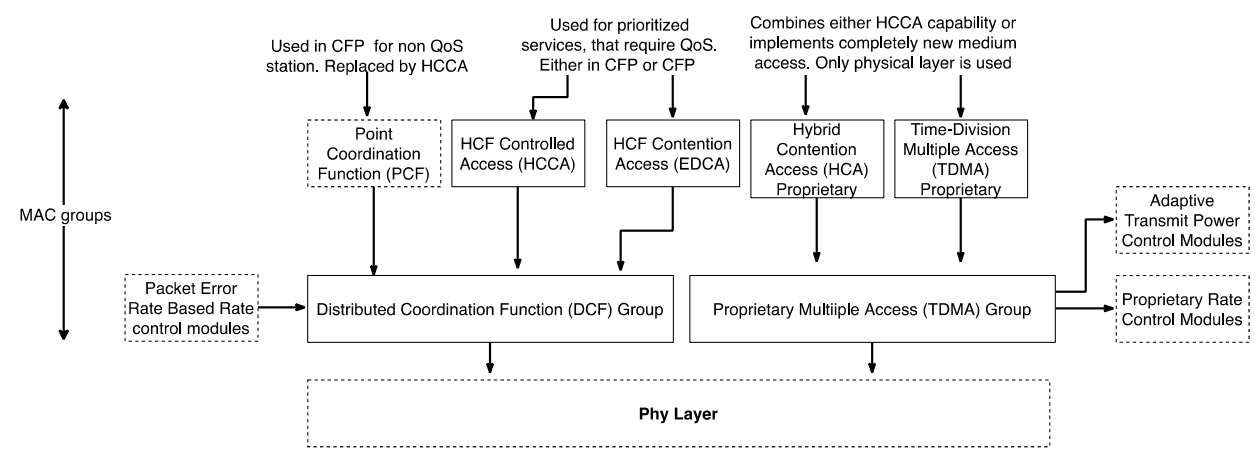

Fig. 1.3. The Medium Access Control protocol groups based on IEEE 802.11 standard 
The Fig. 1.3 lists the standards and proprietary based protocols which are used in State-of-the-art nowadays. The main use-cases, which are considered, are as following:

1. Indoor type - this is a typical scenario, where all the IEEE 802.11 based equipment is placed indoors and usually main requirement is interoperability between user's devices and Access Points. Therefore, none of custom made IEEE 802.11 MAC layer changes happens in between indoor based implementations.

2. Outdoor type - this can be either hotspot based use-case, where public $\mathrm{Wi}-\mathrm{Fi}$ is provided to end users or more advanced scenario where last mile communication is provided to user's. However, interoperability is still a requirement in order to connect different vendor equipment together. Therefore, either HCCA or EDCA versions of MAC layer mechanisms can be used here.

3. Outdoor proprietary type - this is highly advanced closed source MAC. Typically provided by wireless equipment vendors, who build their last mile or backhaul solutions on top of IEEE 802.11 standard. As expected the standardized operation of IEEE 802.11 chipset is changed according to performance gains. Since competition in this field is harsh, operational methods are closed. In this category, it's possible to see different types of IEEE 802.11 schemes, which can be based on EDCA, HCCA or TDMA. Nevertheless, operational methods of these proprietary protocols can still be researched by performing wireless sniffing. Usually proprietary based protocols implement PCF (polling based) approach without Contention Period, since combination of CFP and CP periods increases complexity, therefore only CFP part is being implemented. Additionally, TDMA is being implemented in similar way as PCF, the only difference is a strict timeslot for the connected stations.

Current standard is typically built for one purpose - entertainment in home applications, where a relatively low number of wireless devices (usually up to 10) operate and it's still has enough number of available channels on $5 \mathrm{GHz}$. However, devices, which operate at $2.4 \mathrm{GHz}$ even in home appliances, have performance degradation, since available frequency is limited and shared between non IEEE 802.11 devices. While considering this typical use-case, it's noticeable that EDCA protocol with low density of clients operates quite well. The EDCA is a former DCF with additional QoS capabilities. EDCA has four queues, which are used to separate high priority traffic such as voice or video (Perahia, Stacey 2013). The separation is made in such way, that device has different access opportunities set for wireless medium and this is set accordingly to queue priority. By exploiting different DCF schemes on one of this queue, higher priority traffic is sent without any delays. However, what is not mentioned by the IEEE 802.11 standard, which 
implies to all the previous and upcoming generations of IEEE 802.11 standard is an ability to control airtime for Access Point transmission time for the connected stations. In outdoor based devices AIFS parameters can be adjusted not only to get better video or voice QoE, but also to get immunity against interference which is created by other devices or by multiple connected stations. In this case study scenario considers a high number of stations connected to single AP. At least half of the stations are active - meaning they are either sending or receiving data, which generates throughput higher than predefined thresholds.

The testbed consists of Qualcomm AR9342 chipset based devices with CPU processing power $533 \mathrm{MHz}$. All devices are based on IEEE 802.11n 2×2 MiMo with 64 Quadrature Amplitude Modulation (QAM) modulation capability. Stations that are connected to Access Point have respectively $15 \mathrm{dBi}$ and $20 \mathrm{dBi}$ antennas, while Access Point has 90 degree $18 \mathrm{dBi}$ sector antenna. The distance between clients and AP is $200 \mathrm{~m}$ and $5 \mathrm{~km}$ respectively. The channel width is $20 \mathrm{MHz}$ and frequency is set to $5700 \mathrm{MHz}$. All deployments were done in Vilnius city, where high interference levels can be seen due to already deployed equipment by other users.

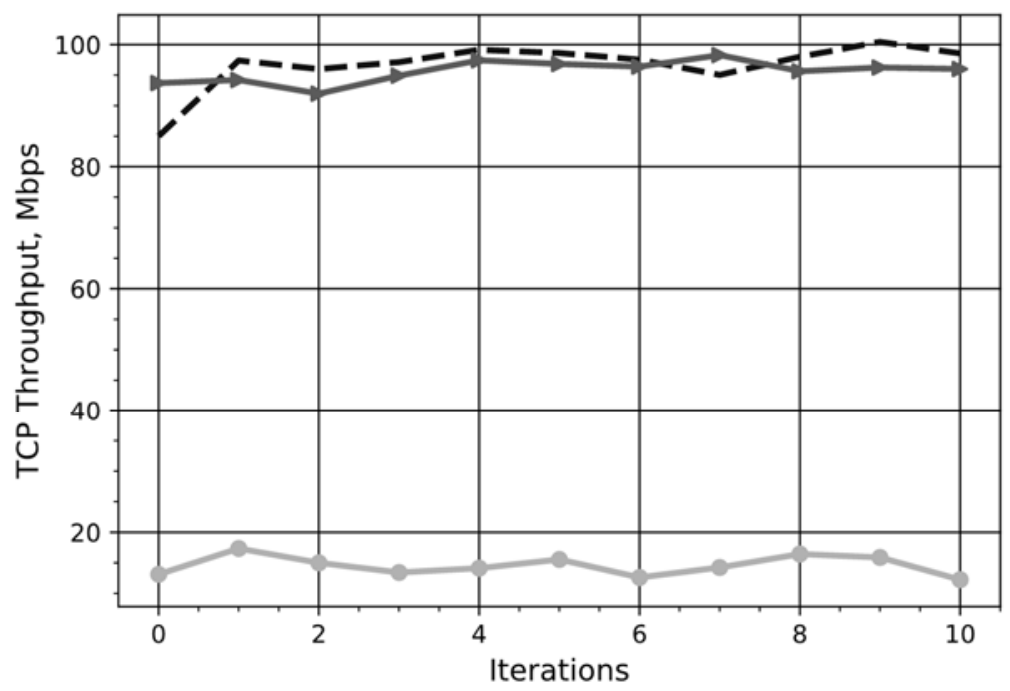

Standard CW and AIFS values for IEEE 802.11e AP CWMIN $=2$, CWMAX $=3$, AIFS $=0$ AP and STA CW and AIFS values set to 0

Fig. 1.4. Transport Control Protocol throughput through 60 clients. Different Wireless Multimedia Extensions settings are applied both on access point and stations 
The test will be started by measuring capacity of one station connected at $5 \mathrm{~km}$ distance. The signal levels are respectively $-72 \mathrm{dBm}$ and $-74 \mathrm{dBm}$ per chain. These signals aren't fulfilling the requirements to use the highest Data Rate. Since Data Rate requires signal levels at least $-65 \mathrm{dBm}$. However, the Access Point can still reach reasonable throughput. Half of the stations are $5 \mathrm{~km}$ away from Access Point, it provides a good opportunity to test different WME settings impact against interference. The testing was started by measuring throughput over station and Access Point with different CWmin, CWmax and AIFS values. CWmin, CWmax and AIFS settings have been changed on both Access Point and station devices. By default AP has different CW and AIFS values compared to the stations, this is because AP has to contend with connected stations as well, therefore to improve fairness AP has higher priority for wireless medium access, this is achieved by using lower $\mathrm{CW}$ and AIFS values compared to the stations.

In the Fig. 1.4 only 60 stations are active, the maximum capacity is reached when the AP WME settings are adjusted from the standard ones to the lower, so AP would issue shorter AIFS before transmitting its data as well as have lower back-off time. It can be noticed, that if station's AIFS is higher than AP's, it is possible to achieve higher performance figure. However, if AIFS values are smaller or equal to AP the throughput decreases remarkably.

Nevertheless, the actual implementation of such priorities still has drawbacks. For example, if video queue contains few video packets marked with high priority and low priority queue like best effort contains normal data. All the packets from video queue will be pushed firstly and if algorithm does not detect any packets in video queue it starts to handle best effort queue. However, while handling overbooked best effort queue, device might receive video packets, while algorithm still handles best effort queue. Thus, video queue will be delayed while transmitting those lower priority packets, therefore EDCA QoS extension is considered not strict QoS, but a pseudo Weighted Round Robin (WRR).

The access of IEEE 802.11e EDCA mechanism works in the same way as former DCF. A station or number of stations are competing for wireless medium, in order to control the possibility of collisions, that are a common thing between wireless devices. DCF has a medium sensing mechanism, virtual and physical of those. The station senses wireless medium if it's busy or not. If medium is idle for Distributed Inter-Frame Space (DIFS), station is allowed to transmit it's data. Otherwise initial back off counter is set. The back off is a binary exponential back off (BEB), which counter is increased uniformly and randomly from a range [ $0, \mathrm{CW}]$ Contention Window. Station decreases this counter only if the medium is sensed idle for a period of DIFS plus back off. Otherwise, back off is frozen if medium is still busy. While staying in back off procedure, the station continues to sense the channel, and as soon as an idle channel is sensed, the station reduces its BC for one-time slot. If back off reaches zero, station is permitted to transmit it's 
data. Upon successful transmission the Short Inter-frame Space (SIFS) follows and station receives The initial value of CWmin is 0 , and it doubles in case the station experiences a collision; otherwise, if a transmission is successful, the value will return to CWmin. Acknowledgement frame. EDCA queues have the following parameters: min and max CW values, Arbitration Inter-frame space, which depending on queue are not less than DIFS.

Since EDCA or former DCF is so common in home and outdoor appliances, there have been many attempts to improve performance of this protocol. There have been more than 500 attempts of various media access control suggestions, which might increase the throughput of standard wireless medium access protocol. One of the ways solve the back off problem (Shi et al. 2010) is to use fixed contention window size. The key idea behind such suggestion is that station reserves back off counter (BC), so it's no longer uniform or random, it's a fixed, and every station can reserve a fixed back off counter. After successful transmission this counter is released and shared between other stations that couldn't make reservations. It is expected, that stations which couldn't make reservations didn't have any traffic or were collision free. During beacon interval (BI) Announcement Traffic Indication Message (ATIM) frames are delivered and received by group of stations. These frames are used to set the static BC and stations can transmit data on different back off intervals. In case there is station with QoS requirement, there is an opportunity to store emergency flag in to the ATIM frame and thus achieve access to wireless medium faster.

In the next attempt (Lee et al. 2015) authors propose an optimal O-DCF, this is done by controlling supply and demand chain of queue buffers. Each transmitter is equipped with additional queue Mac Queue (MAQ), which acts as supplier of data packets to lower layers. By controlling MAQ size authors controls link access aggressiveness by adjusting $\mathrm{CW}$ parameters. The initial $\mathrm{CW}$ size is determined by sigmoid function of the size of MAQ. After that BEB is used to handle collisions. By controlling both parameters authors can handle the collisions and queue length relation, which allows higher performance when compared to standard DCF access methods. However, article seems to be lacking information how the QoS will be handled in case there will be different type of service. Moreover, CW limit as is $\mathrm{CW}=10$. This is a limitation of hardware, therefore BEB will be limited as well. At some point with more than 50 active clients proposed protocol will encounter starvation scenario, since there would be too many collisions even with the limited queue length.

The renewed O-DCF (Lee et al. 2015), added additional improvement and thus the new protocol was called Advanced DCF (A-DCF). The reason for this new protocol is related with the fact, that previous O-DCF couldn't handle TCP sessions. Since TCP throughput is highly related to the fact that TCP monitors 
congestion (Sharon, Alpertb 2016) by increasing the wireless medium access aggressiveness O-DCF created a closed loop, where some of the stations couldn't increase their queue length, because a lot of retries were happening. Therefore, TCP congestion mechanism would decrease window size and throughput would be still low without possibility to increase. A-DCF maintains per-neighbor states, that are used to determine how aggressive the wireless node should compete for wireless medium. A-DCF maintains two queues Control Queue (CQ) and MAQ. CQ buffers the packets that are passed by upper layers and later enquired perneighbor CQ as frames. MAQ plays here an important role, that resolves how aggressively wireless node should compete for wireless medium. Demand Rate Regulator (DRR) is controlling the dequeuing speed from CQ to MAQ. The dequeue speed is directly related with fairness between wireless nodes and thus is a key element of the A-DCF design. The supply demand chain is based on MAQ delay, the longer MAQ is not served the more aggressive the wireless node is made, since wireless node is expected to either have small CW or long transmission length. The aggressiveness provides a chance to increase TCP throughput. The frames which are moved from MAQ to Transmission Queue are sent directly by the chip. While moving them to the lowest layer, transmission TXOP and CW parameters are set, which regulates the aggressiveness.

HCCA is a former PCF with QoS capabilities (Kanda et al. 2016), where central entity performs polling requests between stations. The primary assumption is that APs and STAs are located in close environment, therefore a capture effect can be exposed in order to do a pseudo-sync of multiple APs. Multiple HCCA APs utilize the capture effect and duplicate the timing by inter-AP coordination, which is defined in IEEE 802.11aa. The issue here with this assumption is that authors (Kanda et al. 2016) do not mention how they deal with timing drifting between different APs, because usually clocks between different devices are not synced, overlaps will happen between transmission and reception. This would cause chaotic performance and would cause unstable QoS service to the actual users, especially in hidden wireless node topology.

The authors (Daisuke et al. 2015) propose priority schemes for stations that can deliver packets successfully, they suggest adjusting the AIFS parameter, which allows stations to access wireless medium more aggressively. However, if station fails to transmit packet successfully they lose the priority for wireless medium access and has to contend among other stations. The proposal does seem more like ALOHANET protocol, where stations can access time in random periods. The suggested model wouldn't provide any performance increase in hidden node environment, since by decreasing AIFS parameter, it would cause more collisions, which would result in stations operating at standard Contention Period (CP) environment. However, this might work well in indoor environment, where CCA mechanism would prevent from high collision event. 
Yet another attempt was made by authors in (Sigh et al. 2015). The idea is to re-use RTS/CTS mechanism in more dynamic way, authors propose to use dynamic RTS threshold allocation and provides the simulation results with OPNET tool when varying RTS threshold is used under network topology consisting of 100 wireless nodes. Ibukunoluwa, Yang (2016) implemented dynamic variation of $\mathrm{CW}$ based on wireless environment and number of active wireless nodes in the network. The feedback mechanism which supplies controller with monitored data, after that a controller outputs optimal CW value, this one of the many articles where performance efficiency is related with controlling CW minimum and maximum size. Similar attempt was made by Nasrallah et al. (2016) this time they attempt to control the BEB value.

The idea is to control a BEB according to collision percentage. Authors proposed to evaluate the wireless medium collisions by using Markov-chain analytical model. The model is based on well establish Bianchi (Bianchi 2000) Markov chain model and highly researched by wireless community in many articles with and without additional features. Furthermore, Prasetya et al. (2015) are again exposing the same idea as in many articles before. In this article they are trying to optimize EDCA wireless medium access protocol by controlling AIFS and CW size. This method has been tried by many others and in some cases provides benefits. The clear benefits are seen in Point-to-Point scenarios, however in Point-toMultipoint or typical hotspot scenarios with lower CW and AIFS values users might experience performance loss compared to standard values.

\subsection{State-of-the-art Advancements in IEEE 802.11 Media Access Control}

There have been a lot of suggestions from scientific community, however most of those suggestions are left in articles and rarely implemented on actual embedded devices. Since wireless environment is expanding some of the research ideas or methods are used by global wireless equipment vendors. The advancements can be categorized based on functionality.

First of all, based on the application type advancements can be categorized on outdoor or indoor usage. Indoor IEEE 802.11 devices will typically follow standardized methods of wireless medium access, since this is the only one, which is approved by Wi-Fi alliance.

Some of the vendors implement a band steering technique, that acts as loadbalancer between $2.4 \mathrm{GHz}$ and $5 \mathrm{GHz}$ radios with preference to $5 \mathrm{GHz}$ radio. The implementation of band steering solves inefficient dual band radio usage on home premises. A typical dual band radio does not have any specific rules which radio should be preferred $5 \mathrm{GHz}$ or $2.4 \mathrm{GHz}$. It is expected that most of the devices will 
connect to $2.4 \mathrm{GHz}$ even though there is a possibility to get better performance while connecting to $5 \mathrm{GHz}$ radio. Therefore, this causes under-utilization of one band. Since $2.4 \mathrm{GHz}$ is usually overcrowded, there is a need for load balancer and Qualcomm introduced a band steering feature (Qualcomm's Programmer's guide 2018).

Additional feature is beam forming with multi-antenna arrays introduced by Ruckus Wireless (Ruckus 2016; Ruckus 2013). By exposing beam forming functionality Ruckus system can utilize seamless roaming as well as provide better SNR to individual clients. This is accomplished by monitoring the movement of the clients in the area.

Since indoor requires more complex methods to improve performance and typically not all connected stations can support advanced features. Usually APs are operating according to standard defined rules and thus not much innovative mechanisms can be introduced while still requiring compatibility with legacy devices.

Most of the vendors state-of-the-art outdoor wireless equipment are based on IEEE 802.11. However, vendors build their own wireless medium access protocols as proprietary protocol on top of existing IEEE 802.11 standard. One such protocol is a TDMA based implementation. Since it's not hardware based TDMA, it's called a soft-TDMA implementation and is a trend between wireless equipment vendors. The soft-TDMA system, that receives a packet with original sequence number, creates a queue where these packets are modified by including virtual sequence number in the header of the packet and includes an original sequence number in the payload of the modified packet. This aggregation approach relies on virtual sequence number for one particular reason - inefficient utilization of wireless bandwidth (Dayanandan et al. 2016). The meaning behind this is that standard 802.11 approach requires acknowledgement for every transmitted packets due to error prone environment. However, acknowledging every frame results in underutilization. IEEE 802.11n provides Block-Ack (BA) packet aggregation mechanism, which aggregates multiple frames if all of them are sent to the same destination. Thus, a single transmission unit is created. Standard defines that up to 64 packets can be aggregated, after transmission of such unit, receiver waits SIFS amount before transmitting ACK. Block-Ack sequence number is a direct derivative from first aggregated packet sequence number. The bitmap corresponds to all the packets which are encapsulated in aggregated frame. Block-Ack has a sequential bitmap, therefore only continuous number of packets are acknowledged. The 802.11 Block-Ack has some drawbacks, which include packet aggregation Traffic ID (TID). Hence, when transmitter has more than one traffic category to transmit, efficiency is decreasing (Dayanandan et al. 2016). This is due to reason, that Block-Ack can acknowledge only a group of continuous 64 packets, the retransmitted aggregated frame can't contain any packet with sequence number 
higher than 64. As stated transmission efficiency might vary a lot depending where corrupted packets are located. Discussing further limitation of $802.11 \mathrm{n}$ that aggregation of frame can carry packets only from identical traffic category. So transmitter can send only two packets in video queue and 64 in best effort queue, which means that it will have to access wireless medium twice just to send those two video packets.

Since Block-Ack allows only to aggregate packets of the same category, a situation is created that receiver firstly aggregates those 2 packets and after receiving Block-Ack from receiver again performs the same. However, here incurs double back off and listening period and double SIFS are issued. This results in lower performance due to increased waste of transmission time.

The proposed method by vendor solves the above mentioned problem by using virtual sequence numbers for aggregated frames as well as virtual TID numbers. Basically, all the original sequence number and TID are put into payload of the packet, which is encapsulated with virtual sequence number and 802.11 wireless header updated with virtual TID number. Thus, a multi-TID aggregation mechanism with virtual sequence numbers is built. The mechanism itself gathers all the encapsulated packets with 802.11 header from every TID and aggregates them into one single virtual TID together with virtual sequence number.

However, higher priority packets are moved to the front of the queue for quicker assembly. Because the virtual sequence numbers do not have actual meanings and are only used to allow the receiving station to send a Block-Ack that acknowledges all the packets in the aggregate frame, the virtual sequence numbers can be restarted for every transmission - the transmission can be stateless. Note that the transmitting station might need to retain the virtual-to-original sequence number and TID mapping until the Block-Ack is received in order to identify the correct packets for retransmission when packet corruption happens.

Because of the flexibility afforded by the virtual sequence number and TID, the transmitting station can reserve packet slots in the aggregate frame for redundancy purposes, in order to mitigate non-ideal transmission conditions. Furthermore, the transmitting station can monitor the packet error rate for each TID, and dynamically replicate packets for each TID based on a predetermined QoS policy. When allocating reserved packet slots for duplicate packets, the transmitting station can use various methods (such as strict priority based or round robin) to ensure the desired QoS parameters are met.

Another attempt of creating a more efficient wireless medium access is presented in (Pera et al. 2014). A IEEE 802.11 wireless system consists of multiple stations and single AP. Station can communicate only after received a token, special frame which allows to transmit data. According to this patent it solves deficiency which can be noticed in standard based CSMA/CA approach, in case when 
distance between separate wireless nodes is large enough to disable CSMA/CA functionality.

In basis, AP has two queues, one for more active clients and another one for less active or idle clients. Both of queues are still polled, however the frequency how often one or another queue is polled is different. Therefore, it can be thought that idle queue is polled two times often than an active queue. As stated in patent, between polling those queues stations are aggregating its data and upon receiving a token they can send that data. In patent it's stated that this approach can be used, where more than one station could be polled at the same time. This is based upon probability that less active station won't have any data to transmit, therefore combining single token to poll one active and one idle station at the same time, should not resolve in collision. In article it is stated, that polling frequency can be regulated either based on data or amount of airtime required to transmit that data.

When station is polled it's moved from front of the queue to the back of queue. It's sort of First-in-First-out (FIFO) operation on both queues. When two stations are polled, one from active and another from idle queue (at the same time or with small separation) controller, AP waits for defined period of time to receive response. Upon receipt of response from either queue, AP evaluates the received signal. In case it's from low activity queue AP decides if station can be moved to active queue, otherwise station is still kept in idle queue. The opposite operation is performed with station from active queue. After this step, AP moves to another set of stations on both queues.

Why such mechanism is required in wireless networks, especially in outdoor wireless networks can be explained by following statement. In outdoor wireless networks, since CSMA/CA is not able to cope with hidden wireless node problem, implementations have to rely either on strict TDMA or Polling approaches or hybrid combinations of them. However, sending control frames to station, which have none or very few packets to transmit, increases unnecessary control frames, which takes large amount of airtime, when system has a high number of served clients per single AP. In order to decrease this overhead, system implementation has to rely on smart methods to decrease this, therefore a system can use one of the algorithms to separate active from idle clients and increase airtime usage efficiency.

Some of methods are used to predict station behavior, like a historical throughput values or empty polling values that could allow to predict station behavior in real-time. Based on such information AP might allocate required time to transmit stations data or move between two queues, which were described earlier. Time is stricter method to control stations, since different station might have different signal levels, interference impact and similar effects. 


\subsection{IEEE 802.11ad Standard $60 \mathrm{GHz}$ Advancements for Multi-band System}

IEEE 802.11ad is a new technology, that allows to reach Gbps throughput over wireless both in point to point and point to multipoint network topologies. Since technology uses $60 \mathrm{GHz}$ band it allows to performed frequency reuse due to quick signal fading effect.

While considered IEEE 802.11ad wireless technology and forthcoming Wireless access Heterogeneous networks (WA-HetNet) need to consider implementation costs of such wireless systems, which expands currently LTE-Advanced scope and aims to provide key enablers for $5 \mathrm{G}$ networking. This is especially needed to build a multi-band system. The IEEE 802.11 ad frequencies are not fixed on $60 \mathrm{GHz}$ band only, 802.11ad is already planned to migrate to $30 \mathrm{GHz}, 70 \mathrm{GHz}$ that have lower attenuation and less oxygen absorption. In IEEE 802.11ad systems, SDMA is essential to overcome the large Free Space Path Loss (FSPL) (Nikolikj, Janevski 2015).

High path loss is one of the challenges that multi-band system has to overcome which requires directional high gain antennas compensate for the propagation loss for $60 \mathrm{GHz}$ up to $20 \mathrm{~dB}$ higher compared to lower band ISM frequencies like $2.4 \mathrm{GHz}, 5 \mathrm{GHz}$ (Singh et al. 2009) for long range installations. But high directivity and narrow beam width creates more issues, like carrier sense capabilities decrease, however greater spatial reuse is possible due to high directivity (Feng et al. 2014; Singh et al. 2009; Gong et al. 2010; Singh et al. 2010)

IEEE 802.11ad wireless technology is seen as enabling element of $5 \mathrm{G}$ networking (Nitsche et al. 2014), basically due to its capability to give high throughputs which can surpass 2 Gbps to single user or even higher if channel bonding (Cordeiro 2013) would be available. Moreover, the IEEE 802.11ad could be the key technology enabler for wireless digital interfaces (WDI) and thus replacing typical wired interfaces. Due to short range operation IEEE 802.11ad systems are targeted for Point-to-Point operation and mainly for indoor usage, however in near future a new type of heterogeneous networks will appear where cellular and WiFi networks will cooperate as single entity. Thus, $60 \mathrm{GHz}$ system operation is going be to extended for point to multipoint operation within public area, backhaul or office applications (Urushihara et al. 2014).

The IEEE $802.11 \mathrm{ad} 60 \mathrm{GHz}$ frequency band is becoming popular between small wireless internet providers and backhaul users at the same time it provides some challenges (Hemanth, Venkatesh 2015; Sim et al. 2016) like deafness and collisions between hidden wireless nodes. These are primary research topics, which are escalated by $60 \mathrm{GHz}$ community. However, since IEEE 802.11 ad is not widely spread there aren't any real life tests in point to multipoint deployments 
yet. Most of the articles are working on solutions for deafness problem or investigating possibility of multicast streaming capacity and only few are suggesting some additional MAC layer improvements. Nevertheless, $60 \mathrm{GHz}$ band is expected to participate in wide area of scenarios beginning from wireless entertainment systems, streaming high definition video and even providing backhaul or last mile communications for outdoor scenarios. Therefore, IEEE 802.11ad uses a versatile wireless medium access schemes that combine both CSMA/CA and TDMA operational capabilities (Hemanth, Venkatesh 2016).

The TDMA is widely used in wireless networks, because it provides required capabilities such as hidden node problem solving, bounded delay, energy efficiency and reduction of total system performance impact due to client low rates. All these factors made TDMA one of the key enablers for today wireless systems, even increasing interest in already existing IEEE 802.11ac networks (Feng 2016). The interference for $60 \mathrm{GHz}$ band has lower impact, since typically $60 \mathrm{GHz}$ systems will use high gain directional antennas and most of the interference might occur inside a network. Close proximity wireless deployments will be the usual cause of the collisions between devices and this has to be addressed. Collision typically would be seen on infrastructural networks, where multiple APs are colocated on the same tower, thus spatial reuse is critical.

IEEE 802.11ad standard itself defines a clustering schemes (Fig. 1.5), which are adopted to multiple PBSS Control Point (PCPs) operating on the same frequency and are in the same Personal Basic Service Set (PBSS). One of the key elements in clustering scheme is a synchronized PCP which acts as a main controller for multiple other PCPs co-located at the same place. The members which belong to the cluster selects the same Beacon Interval (BI) in order to transmit beacon at the same period as the synchronized PCP. The starting time of BI in cluster is a ratio between $\mathrm{BI}$ and number of PBSSs. Each PCP sends a beacon time interval (BTI) to transmit frames that contain SP allocated of the current PCP beacon interval (BI), during this period all the other PCPs are in listening mode and not in transmission mode. The Beacon Header Interval, is only used for statistical information transmission, the reversed slots of current SP on PCP, which sends the BHI. Typically BTI are the same between several PBSS. In case interference among clusters are detected, cluster mechanism allocates non-overlapping time periods. Current IEEE 802.11 ad design only one cluster is considered, thus the members of the cluster are allowed to use overlapping time periods.

The standard IEEE 802.11ad provides some challenges as well, like inefficiency of CSMA/CA and some new issues like deafness problem, which occurs basically due to usage of multi-array antennas to concentrate beams towards stations. Since path loss is higher than on lower frequencies, IEEE 802.11 ad has to use Quasi-Omni (QO) antennas. Usage of directional antennas complicates the MAC layer design, however compensates the propagation losses significantly. 
MAC layer addresses deafness problems by introducing TDMA approach, which is used for communication, it's an addition to CBAP wireless medium access scheme. It is known that CBAP (CSMA/CA) is very efficiency when needed to handle burst traffic streams, however CSMA/CA in these deployments partially lacks carrier sense capability due to high directivity of antennas. Quasi-omni means that transmission occurs using directional antenna and not omni as it was popular on lower frequency bands like $5 \mathrm{GHz}$. The directional antennas both enhance and provide possibility for spatial re-use, however at the same time introduces deafness problem, which was researched by Mashinchi-Ghabel et al. (2017).

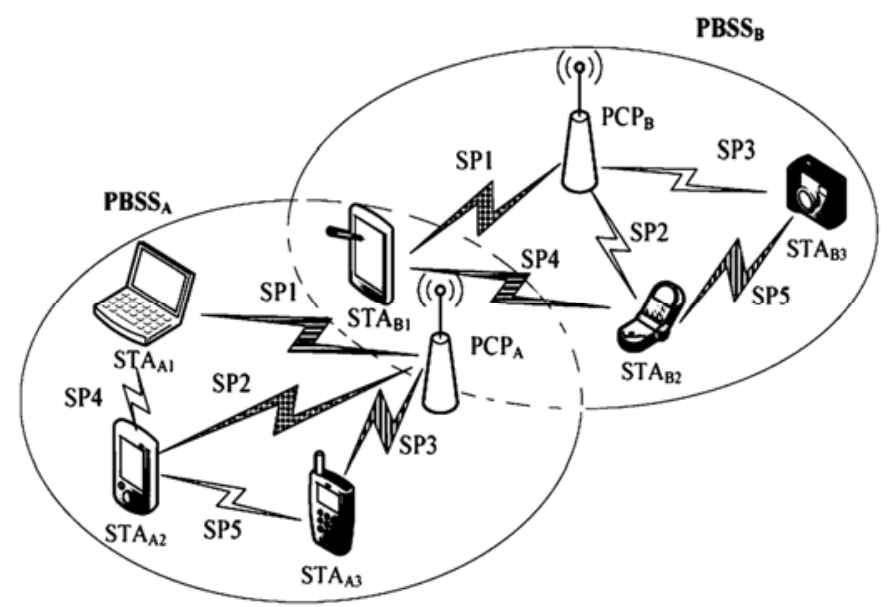

Fig. 1.5. Personal Basic Service Set (Feng et al. 2015)

The problem is mainly related to the fact, that stations which use directional antennas doesn't receive signal from nearby actively operating stations. One of the reason for this is that while PCP tries to communicate with DMG STA. PCP might be tuned its antenna beam towards some other STA device, while STA which tries to communicate but doesn't receive response starts exponential back off, which doubles interval upon unsuccessful attempts, same way as it happens on standard $5 \mathrm{GHz}$ or $2.4 \mathrm{GHz}$ band. Eventually we have a situation that a network performance degrades. In (Mashinchi-Ghabel et al. 2017) authors tries to improve this by suggesting methods like different beam width of antennas. The authors propose to use narrower beam width antennas and combine that with RTS/CTS mechanism to prevent deafness problem. When device has a 360 degrees antennas, it usually will have 12 sectors, where each sector has 30 degrees beam width, assuming that each station is connected to every of those sectors, means while communicating with one of the stations system simply does not receive signal 
from other stations connected at different sectors. The signal is received by PCP only from stations that are connected in the intersection of sectors. Antennas with narrower beam width split the area into 4 equal QO levels, so more stations are gathered on single area and less deafness is possible.

Another approach on deafness issue is pointed out by (Sim et al. 2016). Like in article before, authors tend to expose the existing IEEE 802.11 ad functionality FST. Which allows to send and receive traffic at the same time over different bands in example: $5 \mathrm{GHz}$ and $60 \mathrm{GHz}$. It acts as failover guarantee for wireless device, however in this case authors try to re-use the $5 \mathrm{GHz}$ band as control communication channel to send the RTS/CTS, since $5 \mathrm{GHz}$ can use omni-directional antennas and sent RTS/CTS to all connected stations and this is one of the way for deafness problem. According to (Sim et al. 2016). this is very reliable solution, since $60 \mathrm{GHz}$ is primary used for short range communication typically up to $100 \mathrm{~m}$ per say. However, this is a letdown when considering longer PTMP and PTP $60 \mathrm{GHz}$ outdoor communications, where $5 \mathrm{GHz}$ and $2 \mathrm{GHz}$ band itself becomes a victim of hidden node problem and requires additional modifications. However, for short range and indoor communications this could be a relevant solution, since it decreases excessing back off on IEEE 802.11ad and increases overall system capacity. The dual band schematic provides fairness model which is one of the first solutions for deafness problem. Another important moment is that RTS/CTS typically are sent at lowest rate (MCS0 control rate on $60 \mathrm{GHz}$ ) and this takes away valuable airtime which could be used for data traffic, however by moving all the control frames to the $5 \mathrm{GHz}$ band, authors increase the efficiency of IEEE 802.11ad wireless system. Similar approach was taken by (Mohamed, Sakaguchi, Sampei 2016) where establishment of $60 \mathrm{GHz}$ concurrent links is controlled over legacy $802.11 \mathrm{ac} / \mathrm{n}$, basically it's a controller approach, where an entity decides which of the APs are most suitable for the end user equipment and thus control messages are sent over existing Wi-Fi link.

IEEE 802.11ad introduces some new wireless medium (WM) access schemes such as contention based access (CBAPs) which are used for low priority traffic. The service periods (SPs) are reserved by stations are used for QoS applications, thus a hybrid wireless medium access similarity to IEEE 802.11e can be noticed here. The (Rajan, Babu 2016) researches multi-gigabit station (DMG STA) under different QO throughput saturation. The saturation is based on the Markov chain model (Chandra et al. 2017) and only non-aggregated traffic is considered while modelling. Similar approach is done for Service Period (Hemanth, Venkatesh 2015) that tries to evaluate the performance during service periods.

Since $60 \mathrm{GHz}$ bandwidth can provide high speed communications both outdoor and indoor, some of the authors tries to evaluate streaming capabilities of $4 \mathrm{~K}$ video over $60 \mathrm{GHz}$ bandwidth, such tests were performed by Abe, Walker (2016) on the table by connecting two PCs over IEEE 802.11ad, since distance between 
devices was at maximum $9 \mathrm{~m}$ they didn't see any video quality degradation. Both Point-to-Point and Multi-Hop scenarios were verified and conclusions is that for indoor short range IEEE 802.11 ad can handle several number of $4 \mathrm{~K}$ video streaming at the same time. Most of the articles are testing IEEE 802.11 ad either on very close distance $9 \mathrm{~m}$, which doesn't show if IEEE 802.11 ad can be used for outdoor long range communications.

Yamada et al. (2016) tries to evaluate off-the-shelf devices performance in various scenarios, most of these are indoor based testing cases. The conclusion in article states that performance of 802.11 ad is highly dependent not only on the APs location and angle, but also on the efficiency of rate control algorithm. Additional performance measurements were done based on the distance and signal level, to summarize authors evaluated the built-in rate control algorithm performance based on various locations and placements of the STA and AP/PCP device. Similar article appeared with TCP performance evaluation over IEEE 802.11ad, authors measured similar RSSI, PHY rate variations, even the same measurement equipment is used as before, since there are only few vendors who build 802.11 ad chipsets. It was pointed out, that rate control algorithm can't be built mainly on RSSI parameters, additional indicator is required to have more intelligent rate adaption algorithms. To summarize, article states that TCP performance can't be predicted from lower layer metrics (the PHY rate metrics). The rate adaption algorithm (Saha et al. 2015) failed to provide stable PHY rate for the given distance and thus due to high variability it couldn't keep constant TCP flow.

Another feasibility study was performed in indoor environment, authors of (Saha et al. 2016) run several performance tests in different locations, like other they recorded signal level PHY rates relation while measuring TCP throughput over the wireless devices. The TCP throughput reached nearly $900 \mathrm{Mbps}$ limitation of 1000BaseT Ethernet interface. They concluded that high speed throughput over $60 \mathrm{GHz}$ can be established even through glass or dry wall, even though such elements PHY rate sustained 1540-3080 Mbps data rate. It was also concluded, that $60 \mathrm{GHz}$ attenuation due to distance closely follows Friis model. It's been concluded that high number of reflections makes performance unstable and it's hard to predict the results in environment, which causes multiple reflections and this is trademark of single carrier PHY (Saha et al. 2015). Similar effects have been pointed out by another authors in (Zhu et al. 2012) which states that STBC provides the best results due to its robustness, since data is duplicated, therefore transmission reliability increases.

The next article Is expanding area of the testing and tries to rectify the usage of $60 \mathrm{GHz}$ band for passive optical networks expansion (Kalfas et. al 2016). In this case $60 \mathrm{GHz}$ is looked as viable solution for fiber extension in backhaul scenarios. The $60 \mathrm{GHz}$ is primary targeted as radio over fiber (RoF) solution, how- 
ever RoF requires (Guillory et al. 2011) new approach for wireless medium control and thus authors propose their own medium transparent approach (MT-MAC) which performs negotiation between radio and fiber optics, called Fiber Wireless (FiWi). However, considered that $60 \mathrm{GHz}$ band has a big path loss and typically suffers from oxygen absorption or rain it's hardly reliable for backhaul fiber optics solution unless coupled with extended failover mechanisms.

Another approach on MAC layer improvement was suggested by Feng et al. (2016). The authors propose spatial time division multiple access protocol (STDMA), which provides better spatial reuse strategy. The mathematical model was presented, which is used for multi-cluster mechanism, consisting of more than one Personal Basic Service Set (PBSS). Since $60 \mathrm{GHz}$ band has only four available channel, clustering and spatial reuse is one of the key elements in order to build high performance systems. Spatial reuse is a functionality that is followed both by $5 \mathrm{GHz}$ and $2 \mathrm{GHz}$ wireless systems and has been adopted long ago in conventional cellular networks.

The next article tried to suggest similar MAC (Feng et al. 2014) implementation as STDMA, where a database consisting of all interference sources would be available. Then a scheduling algorithm would be used to split the time for each network. The main idea as before is to find a pairs of devices, which can transmit at the same time, since $60 \mathrm{GHz}$ system uses directional antennas, some of the pairs (PCP and STA) can communicate at the same time as the pair in distant proximity. However, clustering which is standardized by IEEE 802.11ad tries to decrease interference only between several PBSS and unintentionally might cause inside its own PBSS, when STA to STA communication is in process. Therefore, authors propose additional field in the training report, which is done before performing synchronization in cluster. The field contains information of the links in other PBSS which can communicate, coexist at the same time. After such report is broadcasted, every PCP in the cluster has a complete database of interfering pairs. S-PCP has an ability to restart the training of interfering pairs detection when interference is detected. Another approach on TDMA (Li et al. 2014) they rely on existing 802.11 ad polling implementation, which schedules the connected clients and provides basic timing. Meaning all the STAs respond, send data only when receive a grant frame.

In last article, authors as well tried to do full TDMA MAC design for IEEE 802.11 ad systems, similar approach is taken based on existing TDMA implementations that are already existing on $802.11 \mathrm{n} / \mathrm{ac}$ systems. However, there aren't any details how the synchronization will be kept in their TDMA implementation since TDMA usually uses GPS synchronization approach even if the system uses Synchronous Ethernet it's not guaranteed that stable clock generator will be available over wired networks. Also if it's mainly indoor based system, there's little or no use for TDMA based approaches since it requires additional synchronization 
packets and control which increases airtime occupation and less time for actual data, common polling based protocols for indoor would provide all the necessary performance requirements. However, such approach for $60 \mathrm{GHz}$ infrastructural topologies would be highly beneficial and would increase and expand possibilities of HetNet networks (Sum, Harada 2012).

Another MAC layer proposal was introduced by (Chen et al. 2013) the main aim of this protocol is to improve the uplink performance of 802.11ad, since the standard 802.11 ad lacks the way to deal with hidden wireless node problem when Quasi-Omni antennas are used for $60 \mathrm{GHz}$ system. Moreover, usage of RTS/CTS is primary aimed at omnidirectional transmission and not for directional as it's done on $60 \mathrm{GHz}$ (Chen et al. 2013). According to authors, IEEE 802.11ad lacks multi-rate support and thus station-station communication would be possible only with low rate, however this statement is no longer valid, since most of the $60 \mathrm{GHz}$ systems now implement rate adaption algorithms and can use multi-rate capabilities. However, the common deafness problem on the QO is still present (Zhou et al. 2009). Authors focus on uplink during CBAPs period, when every connected STA contends for wireless medium and stations can cooperate with each other, in case a relay with additional station in the middle can increases performance. However, this type of solution works in ad hoc type of 802.11 ad scheme, otherwise it would be impossible for single DMG station to keep connection to main AP and relay data to another DMG station.

Authors of (Zhu et al. 2014) tries to enhance Block-Acknowledgement mechanism in order to adapt it dynamically based on the queue length and traffic flows. Their suggested change is tested in typical DCF wireless access environment, most of Block-Ack adjustments are already widely analyzed in articles referring to standard IEEE $802.11 \mathrm{ac} / \mathrm{n}$ protocol. Some of Block-Ack adjustment are considered as proprietary and are patented, which already has similarities to the proposed enhancement by (Zhu et al. 2014) many before already tried to establish Block-Ack dynamics based on channel conditions and similar results were acquired. The enhanced BA won't suffer from lost subframes and thus aggregation efficiency will be higher. The idea is that transmitter send information about each aggregated subframe to the receiver and this is later used for acknowledging the packet. However, this increases overhead, which in point to multipoint would impact overall system's capacity (Liu et. al 2016).

Aggregation is an important factor for wireless network performance and efficient aggregation mechanism is defining the overall system capacity. IEEE 802.11 ad employs both AMSDU and AMPDU aggregation mechanisms, however they are not very efficient and lacks the dynamics based on channel conditions. The authors Assassa et al. (2016) investigate in which cases the wireless system has to increase the delay before transmitting the queue in order to do higher level aggregation. Authors propose an uplink restrictions based on packet count or the 
timeout value, whichever comes first. However, this is not a new technique and is adopted in almost all state-of-the-art $802.11 \mathrm{ac} / \mathrm{n}$ devices. Too big timeout increases the link latency, which has impact for services like VoIP (Guo et al. 2007; Chávez-Santiago et al. 2015) or real-time data transmissions like VR video games (Jiang et al. 2014). The benefit which is stated by article is for bursty traffic flows that is typical for internet traffic. It's also important for uplink, higher aggregation means STA can send more date on single wireless medium access attempt and it's avoiding high contention situation, since STA contends less often for wireless medium (Assasa et al. 2016).

Since rate adaption algorithms are not specified by standard most of them are implemented as proprietary and thus it's typically either PER based rate control or RSSI or a combination of both of them. Similarities to lower frequency band used rate are applied here as well. Kim et al. (2012) tries to implement a fairness based rate adaption algorithm for multicast streams, that are used for wireless video streaming. Since most of multicast traffic streams are sent by lowest rate Modulation Coding Scheme (MCS) it occupies significant airtime, however it still doesn't guarantee that it successfully reaches every client, since it's sent as broadcast stream without any retries in case one or another station device doesn't receive certain packet. The data is sent as broadcast, however based on the simulation results it's assumed to be an efficient way to deliver video stream, however this is possible only in very short range and where isn't any interference. Similarly, (Kim et al. 2011) authors create a relay based network topology to deliver network streams, this is basically done to increase probability of video stream reception since $60 \mathrm{GHz}$ as mentioned before has a high path loss. However, relaying is only good if it has an ad-hoc based network, otherwise in infrastructural topology it wouldn't be able to do traffic stream relaying. Another attempt was by suggesting grouping methods for multicast streams, called incremental multicast grouping, which adaptively determines the beam width and data rate. Basically the incremental multicast grouping does rate selection based on the STA location (Park et. al 2013). In (Wang et al. 2015) authors propose to use multi-leader based multicast approach, which idea is rather simple. In network topology they select several leader wireless nodes, which does the multicast broadcasting to the nearest STA devices in order to increase reliability. Overall, there are several types of multicast protocol, the ones which are converted to unicast are considered as ACK based protocols, the single leader protocol are considered as primary node which sends the multicast to all connection devices and multi-leader which is proposed. When all wireless nodes starts to retransmit the multicast packets and there are several of those, this would increase interference probability and since multicast is typically sent at lowest rate, that would occupy airtime, that should degrade the system performance. 
Most of the IEEE 802.11ad performance highly depends on the channel properties and signal level, reflections and position of the devices. This has been experimentally investigated by Lomayev et al. (2016) who compared experimental and IEEE 802.11ad defined channel model properties. Authors used a laptop docking station with open lid to install the $60 \mathrm{GHz}$ equipment for measurement, the methodology included several different laptop locations, the Line of Sight (LOS) links typically had 25.6 dB SNR while Near Line of Sight (NLOS) had around $13.7 \mathrm{~dB}$ with deviation up to $3 \mathrm{~dB}$.

\subsection{Power Control Algorithms in IEEE 802.11 Systems}

In the past years, there have been several attempts to improve power allocation for 802.11 networks. In (Elbatt et al. 2000) the proposal for ad-hoc network consists of wireless nodes, that operate on very short ranges. By limiting the connectivity range it tries to resolve the interference issues between different wireless network nodes. The connectivity range is a dynamic value which is changed based on the throughput results, after $t$ period the algorithm checks if by increasing connectivity range it can increase its throughput. As long as increasing $N$ range increases throughput of wireless node, connectivity range is increased. If throughput becomes lower, the node returns to previous $N$ settings.

By using lowest possible power in order to decrease the coverage area, the proposed algorithm might create a hidden wireless node problem, also it's worth to mention that this algorithm might be useful only for ad-hoc networks and not for point to point or point to multipoint topologies. In (Broustis et al. 2007) the research of optimal power allocation mechanism is performed. It was observed that maximum power does not necessarily improve the maximum network throughput. The devices which operate on the same channel with different transmit power, only one link will achieve maximum throughput on the operational channel at the same time. However, by using potentially accurate power settings it was noticed that overall throughput was doubled with correct power allocation mechanism.

In (Singh et al. 2002) transmission power is controlled based on the direction of the TCP flow. Power adaption considers the packet loss that can be detected either by reception at TCP sender triple-duplicate acknowledgements (four ACKs with same sequence number) or via timeouts. 


\subsection{Conclusions of Chapter 1 and Formulation of Thesis Tasks}

1. There have been many attempts to improve the quality of service for realtime applications. A few of the solutions suggested a combination of fair scheduled algorithms, while others proposed proprietary based TDMA algorithms. The TDMA based protocol is focused towards scaling capabilities as well as stability and low jitter, which is needed for real-time applications; however, it has drawbacks in traffic flow management and typically can't handle unpredictable traffic bursting in Wi-Fi environment. Therefore, a more robust wireless medium access scheme is required.

2. Most of up-to-date solutions considers very limited operational environment, typically only one type of traffic is considered and thus none of the before proposed solutions can handle triple play services. So far there have been many proposals on the IEEE 802.11 improvement, however most of them aren't fit for point to multipoint in infrastructural networks.

3. Wi-Fi itself is spectrally inefficient and suffers from highly erratic quality of service in congested environments. The ISM bands are treated as a free-for-all; consequently, they are prone to the "tragedy of the unmanaged commons", with their utility being constrained by uncontrolled overexploitation and a lack of coordination among radios. IEEE 802.11 lacks of proper power control, most of the time devices either operate on maximum available transmission power or tries to reach the predefined signal threshold, which causes further performance degradation and thus decreases total system stability.

4. There hasn't been any proposal on the Multi-band Wi-Fi system as a gateway for 5G network offloading. Most of the IEEE 802.11 ad considerations are typically done either by running simulations and thus there aren't any practical experiments which would consider IEEE 802.11ad as a point-to-multipoint or point-to-point high throughput system for backhaul or infrastructural networks. Additionally, there isn't any research done on leveraging IEEE 802.11 ad FST capabilities for active idle client steering, that would bring higher capacity for overall Multi-band system. Active FST usage which would not only do failover operation as defined in the standard, but also would perform active client steering based on their traffic patterns and throughput requirements would increase overall system's capacity as well as higher user satisfaction. Therefore, more research has to be performed regarding active FST steering capabilities that aren't yet defined in the IEEE 802.11ad standard. 
The following tasks have to be solved in order to achieve aim of thesis:

1. To perform detailed theoretical and practical analysis of IEEE $802.11 \mathrm{Me}-$ dia Access Control Protocol.

2. To propose a IEEE 802.11 MAC layer changes, which would increase performance in outdoor based point to multipoint IEEE 802.11 networks.

3. To perform comparative analysis against up-to-date existing IEEE 802.11 standards.

4. To implement a modification for multi-band system in order to increase existing IEEE 802.11 ad system efficiency by using multiple unlicensed bands with active client steering capability for FST function. 


\section{2}

\section{Theoretical Analysis of Multi-band Wireless System}

This Chapter investigates the components of IEEE 802.11 MAC layer adjustments. The tasks for this chapter are:

- Perform a detailed theoretical and practical analysis of open and proprietary IEEE 802.11 Media Access Control Protocol

- Propose IEEE 802.11 MAC layer modification, that would increase efficiency in high density deployments

The material presented in this chapter was published in (Cuzanauskas, Anskaitis 2015b; Cuzanauskas et al. 2015c; Medeisis et al. 2014a; Cuzanauskas et al. 2014b; Cuzanauskas, Haddad 2017; Cuzanauskas 2017b) and presented in five international conferences: (Washington 2014), (Oulu 2014), (Vilnius 2015), (Dubrovnik 2015), (Palanga 2017).

\subsection{IEEE 802.11ad Multi-band Definition}

In order to build a Multi-band capable device several items have to be improved. The first one, is the wireless medium access scheme. There have been several 
attempts of providing better wireless medium access techniques that were mentioned in previous chapter, however most of the techniques requires complex modification of IEEE 802.11 MAC layer and aren't feasible to implement on offthe-shelf device. Additionally, each implementation requires to build software based acknowledgement mechanisms, that aren't fast enough for off-the-shelf devices. Therefore, a new wireless medium access scheme has to re-use most of the features which are provided by the off-the-shelf devices. In example, the hardware retry mechanism, provides faster response when the packet is not received. Furthermore, the features such as multicast traffic translation from multicast to unicast provides better possibility to provide video services, the triple play services. The HCCA access scheme provided by ratification of IEEE 802.11e isn't popular due to its complexity, therefore there is a need for less complicated and efficient wireless medium access scheme. In order to make use of wireless medium access scheme the rate control algorithm has to be capable to adapt to changing environment.

Furthermore, the proper power allocation and usage of highly directional antennas are a key element to decrease the interference impact for wireless networks. Since current wireless equipment is limited under strict rules, some of the frequencies have limited transmit power. Moreover, recent efforts to re-allocate the lower frequency bands, showed that industry requires less strict rules for transmit power usage. This would increase the current devices propagation characteristics together with increased total capacity of the wireless links. However, in order to take advantage of lighter rules for unlicensed band, equipment should use smart power allocation mechanism to introduce fairness between devices. Finally, a proper multi-band system switching based on real-time throughput requirements between several unlicensed bands such as $2.4 \mathrm{GHz}, 5 \mathrm{GHz}$ and $60 \mathrm{GHz}$ is a mandatory to build a efficient Multi-band system and this is the next wireless market approach between State-of-the-art devices and other wireless equipment vendors.

A device is considered Multi-band capable if one more members of BSS are Multi-band capable. The capability is advertised upon connection. Information is included in beacon frames, probe request or probe response frames, which are being sent by Access Point and Client that tries to establish a connection. The IEEE 802.11 ad specifies a FST which maintains the connected station and allows it to move between different bands depending on environmental and capacity conditions. The standard clarifies that FST is initiated when an initiator and responder are identified by FST identifier. In each band or channel, the multi-band capable device has a choice to use either of the supported bands based on its needs with the same MAC address or different MAC address per band. The IEEE 802.11 ad defines two standards: the transparent where the same MAC address is used for different frequency bands and non-transparent if different MAC address are used 
for different frequency bands. Typically device will support the non-transparent mode, since IEEE 802.11 ac and IEEE 802.11 ad are separate radios in most cases.

While the actual IEEE 802.11 ad does not define the actual process of the implementation of hardware, the Linux Kernel contains a appropriate drivers to aggregate or do failover between multiples interfaces. The driver called bonding, executes different operations which can be: aggregation, failover, logical XOR when different frames are transmitted on different interfaces based on their recipient MAC address and similar.

\subsection{IEEE 802.11e Wireless Medium Access Control}

Contention based (EDCA) mechanism is popular between wireless medium access schemes. That's mainly because of ability to work in distributed fashion without external control from main network node. However in most cases contention based mechanism can't guarantee traffic delivery. Even the EDCA that implements separate QoS queues for different type of service can't dynamically adapt to the available channel bandwidth. The EDCA supports several Access Categories, that are meant for different priority traffic streams, from the very lowest traffic type such as web surfing up to the highest voice service. The state-of-the-art devices currently uses EDCA and implements different AC queues for such traffic type. The difference between each traffic category is the way how they access the wireless medium. If wireless medium is busy, the AC depending on its AIFS value start to decrement the back off timer only after wireless medium is sensed idle for AIFS period. If medium during sensing is being active the back off timer is not decremented anymore and continued again after wireless medium is idle for AIFS period. Therefore for any station in wireless network to start a transmission the wireless medium has to be idle.

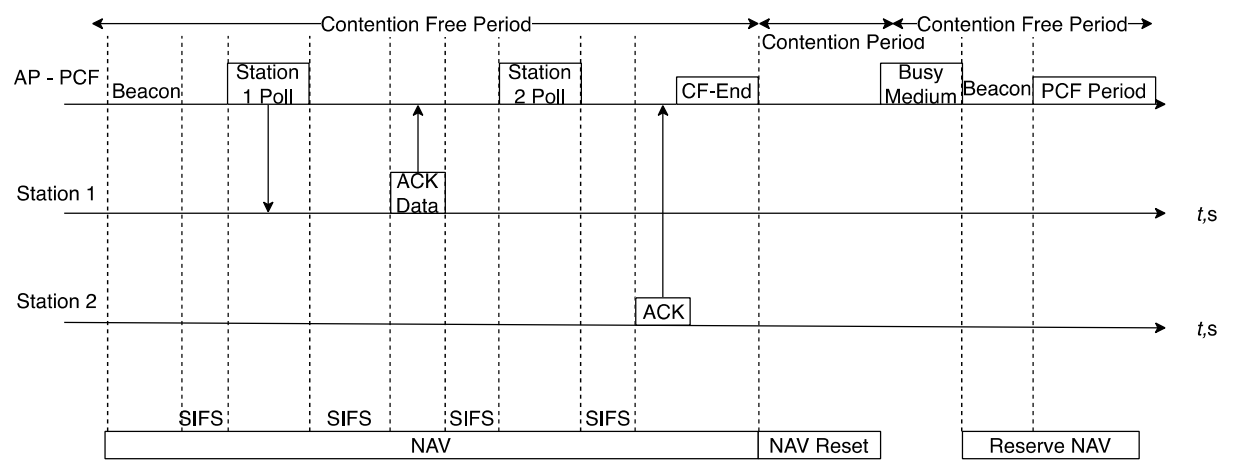

Fig. 2.1. IEEE 802.11 Point Coordination Function wireless medium access scheme 
The PCF uses a coordinated approach and requires stations to send only upon receiving a token, since it's not used widely it became obsolete and was replaced by the Hybrid Coordination Function (HCF) (IEEE Std 802.11-2016).

The PCF in Fig. 2.1, uses Contention Free Period (CFP) to access the wireless medium, in this case AP becomes as main controller and validates any access for connected stations in the same Basic Service Set (BSS) area. The beginning of CFP starts by listening to wireless medium for PIFS duration, the transmitted beacon indicates the Contention Free period and the Delivery Traffic Indication Map (DTIM). After beacon transmission AP waits for SIFS interval to receive either any Contention Free (CF-Poll) frames or performs its own transmission, if there is no transmission CF-End is being sent to close the CF period and NAV will be reset on all the stations participating in the same Basic Service Set area. At the beginning of CFP each station updates it's NAV according to wireless medium access rules, which are being set in the beacon frame by the AP. This is especially important to avoid any type of collisions during CF period between the stations, especially the ones which are operating under hidden node terms (IEEE Std 802.11-2016 ). If station is able to respond to CF-Poll it is allowed to transmit one MAC Protocol Data Unit (MPDU) frame and receive Acknowledgement from the AP upon successful transmission. In case several overlapping APs using this approach operates on the same channel, there are certain restrictions, which implements the usage of randomized back off counter usage and DIFS period for each of the AP to determine if the wireless medium is idle. However as standard defines, there is no permanent protection against collisions. Same can be said for the HCCA mechanism, which as well implements similar approach for wireless medium access.

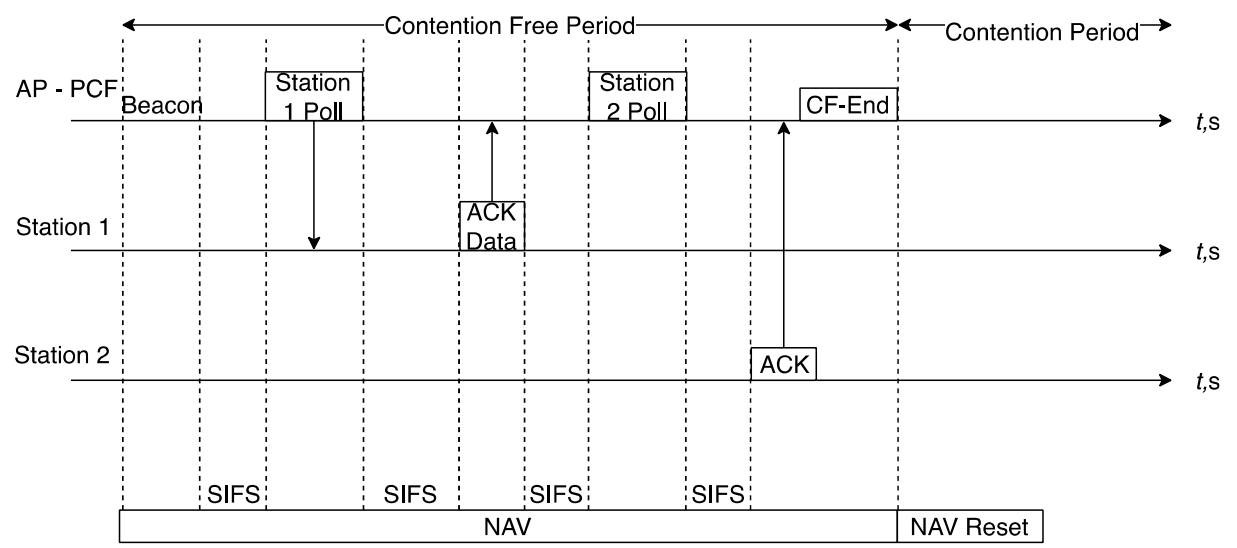

Fig. 2.2. Point Coordination Function and Enhanced Distributed Channel Access scheme 
The PCF and EDCA interactions are seen in Fig. 2.2. after Contention Free Period AP issues an CF-End frame, which indicates the end of Contention Free Period in PCF wireless medium access scheme. During the contention based access period, stations are allowed to access medium by using standard listen before talk approach.

The main inefficiency of PCF approach is that stations without data are still transmitting Null Frames back to the AP and thus occupies wireless medium without actually sending any data, this results in higher delays and slower operation of entire wireless network, therefore PCF is rarely being used in State-of-the-art devices (IEEE Std 802.11-2016) and only EDCA part is being implemented in the most recent IEEE 802.11 devices. Thus, so far only proprietary based approaches tries to implement different wireless medium sharing techniques based on PCF, however such implementations usually relies on Contention Free Period without Contention Period in order to simplify the implementation on the device. The EDCA as mentioned before defines four access categories, that provide QoS capability for different type of services.

The next wireless medium access mechanism is HCCA. The HCCA manages the wireless medium access priority for stations by using both CP and CFP periods. The access for stations is granted upon providing the TxOp, which has a specified duration in the QoS CF-Poll frame. This allows station to transmit more than one frame, while in PCF it was allowed to transmit only one frame. Additionally, HCCA uses the same NAV field to prevent stations from transmitting during this period as well as using the back off procedures if the interference is being detected (IEEE Std 802.11-2016).

The HCCA gains access of wireless medium by using shorter waiting time between transmissions compared to EDCA. The basic operational principal is showed in Fig. 2.3. The standard states that HCCA operation might include specific elements in the transmitted beacon to change the BSS status from distributed access to a point-coordinated BSS and all the stations operating in such BSS starts to set their NAV according to the element in the beacon frame. This limits the contention between the stations and access is granted only if the station is being polled. IEEE 802.11e HCCA might use both CP and CFP periods, in example during CFP period Hybrid Coordinator (HC) can issue a QoS based CF-Poll frame which allows to interrupt the normal CFP sequence and transmit higher priority packets, same approach can be done during CP period. Therefore, HCCA is using a Controlled Access Phase (CAP) to access wireless medium whenever the higher priority packets need to be sent, which is far more complicated packet queuing mechanism compared to IEEE 802.11 PCF. The HC also defines that TxOp for any connected station won't be longer then Target Beacon Transmission Time (TBTT), which a time interval between two beacon transmissions. The end of TBTT finishes HCCA TxOp and normal EDCA procedure continues. 


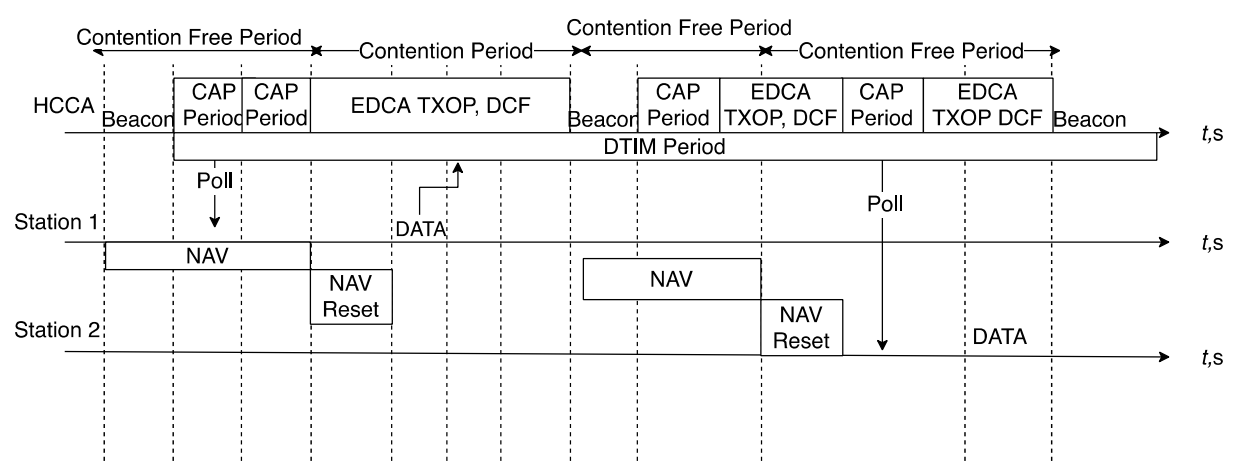

Fig. 2.3. IEEE 802.11e Hybrid Coordination Function Controlled Channel Access scheme

It can be summarized that wireless medium access scheme in HCCA is defined by these principles:

1. After each TBTT, first frame is beacon, which indicates and sets NAV period for all the stations.

2. The CFP period begins after HC senses wireless medium and gains access to WM. The CF-Poll frames are issued for the stations which are polled in sequence according to their connection time, assuming that there is no high priority traffic. HC set its own NAV to prevent any transmission while TxOp is granted to the polled stations. If there is no data or any management frames NAV is being reset on $\mathrm{HC}$ and the connected stations.

3. The next holder of TxOp, before transmission waits a SIFS interval before beginning its own transmission. If the $\mathrm{HC}$ senses that the $\mathrm{WM}$ is idle, it can reclaim the WM.

4. The connected station can use RTS and CTS mechanism to claim higher WM protection against other operational BSS in the same channel.

5. The Controlled Access Phase finishes when the HC does not reclaim the WM and EDCA Contention Period begins. However it needs to be pointed, that during $\mathrm{CP}$ period $\mathrm{HC}$ can still interrupt the process by issuing QoS CF-Poll frame to acquire access for higher priority transmission.

6. The last DTIM frame is used to indicate the multicast and broadcast data delivery for all the stations that are in power saving mode.

HCCA mechanism is the latest IEEE 802.11e proposal and is one of the most complex ones, which in fact is one of the reasons why none of the currently existing state-of-the-art implementations have an fully operational HCCA WM access scheme. 


\subsubsection{Time Division Multiple Access}

In IEEE 802.11 wireless networks TDMA is rarely used, however some of bigger wireless equipment vendors who specialize in backhaul and outdoor point to multipoint tend to select TDMA as a framework for their wireless medium access. Most of these solutions are closed source without further access. The open source TDMA approaches are typically implemented only in modelling tools and not in the actual hardware to the complexity and compatibility reasons in the off-theshelf devices. TDMA, splits the available time into many timeslots and subtimeslots (Djukic et al. 2012). Every new station which connects to main master AP before transmission has to synchronize its internal timer, so all stations would have the same knowledge of a time. The requirement of synchronization is very strict and requires accuracy of microseconds. Therefore, most of the vendors tries to build the hardware with GPS module in main master device to have better time granularity. For each period, every station have a predefined timeslot, thus it can transmit only on that timeslot and must not overpass the predefined timeslot boundaries. This timeslot is used both for direct data transmissions and for retries, which might occur in harsh wireless environment due to interference from outside devices.
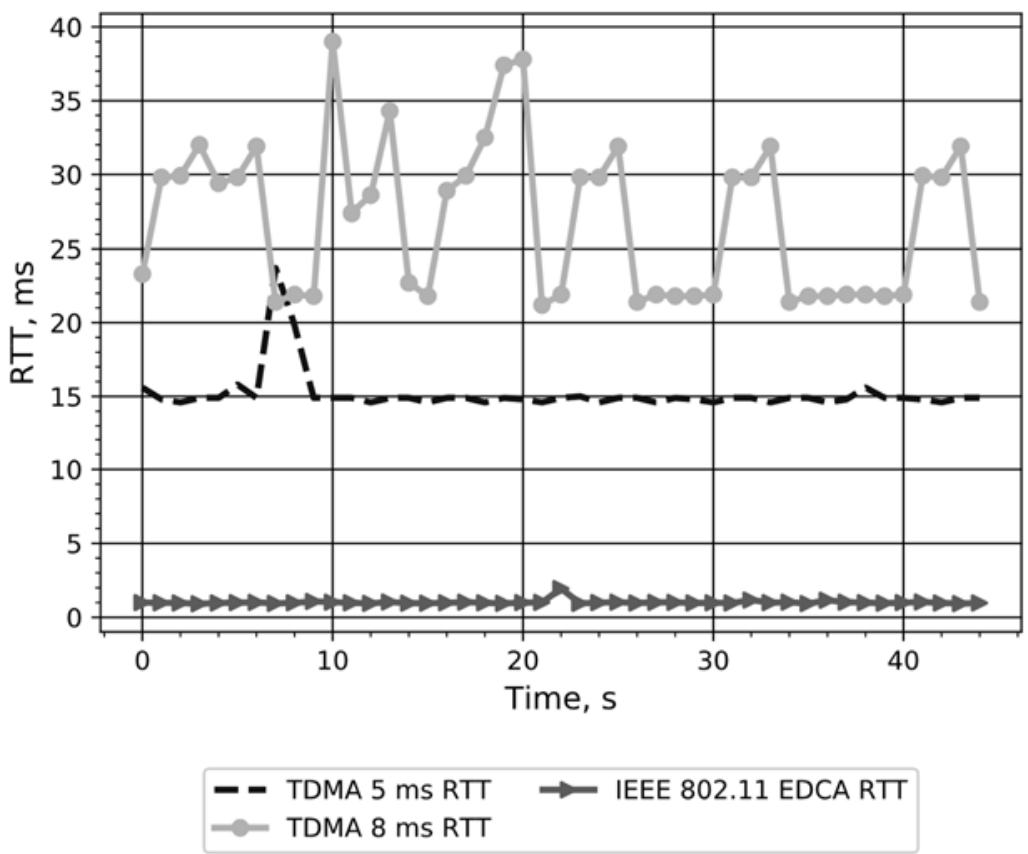

Fig. 2.4. Time Division Multiple Access latency with different time slots 
The advantage of TDMA is that it strictly operates with time rather that queue length based on packet number or bytes. This allows to have smaller jitter between transmissions as well as limits station based on its data rate capabilities. Therefore, every station has a fair access to wireless medium. Thus, stations with low Signal Noise Ratio won't decrease the total system capacity as it's usually seen on EDCA systems. Since stations are allocated fixed length timeslots and in case there is no actual data to be transmitted, the allocated timeslot will be wasted. Similar issue can be noticed on PCF based system. However, by using smaller frame sizes, the issue can be partly resolved and latency would be decreased compared to longer frames. However, smaller timeslots means it is possible to add less data packet and thus limit the maximum throughput.

Due to fixed timeslot length, TDMA usually has a stable, but higher than typical EDCA system latency as can be seen in Fig. 2.4. This is a trademark of fixed frame TDMA system.

While it was mentioned that round trip time is rather stable on TDMA system, it might show opposite result in environment with more equipment as retry mechanism is solely based on software retry algorithm instead of hardware based and thus retries are only performed on the next period of time, when a timeslot for station which will retry packets is assigned again. The reason for software based retry mechanism is that there is no proper way to control the timeslot length in off-the-shelf devices, that uses hardware based retransmits. Same approach is done for IEEE 802.11 PCF modification on top of currently existing IEEE 802.11 EDCA.

Another trademark of TDMA is that it can operate in Near Line of Sight environments (NLOS), this is possible by using guard interval (GI) which allows to separate start and stop of transmission from wireless node. With this type of frame control it is possible to set the maximum distance at which the devices will still be able to connect to master AP. The process of connection is as following:

1. Station device does not send any control frames to wireless medium, until it's connected to master AP.

2. Station operates in scanning mode and checks if there are any master candidate to establish connection

3. AP master sends a predefined timeslot during which connection state machine can execute the association of the client device. This is done quite often therefore connection is far faster than PCF based approach.

One of the very first attempts to bring TDMA in actual 802.11 hardware was made by Leffler (2009). The reason why TDMA was better suited for wireless outdoor usage was that standard EDCA based devices are meant for usage in indoor, where they can hear each other. When devices are placed at different places and distance is more than $1 \mathrm{~km}$, a hidden node problem occurs and no matter what 
optimizations are made to decrease queue length or limit/increase CW size, EDCA will still be lower in performance compared to HCCA or TDMA based solutions.

Since (Leffler 2009; Djukic et al. 2009) there have been many attempts in IEEE 802.11 to increase efficiency of TDMA slot allocation. Xuelin (2015) propose a multi-step slot allocation with multi-phases. First phase Control Sub-Frame phase is a standard DCF, during which all contending wireless nodes tries to allocate a required timeslot for their data transmission. In other words, sends a small request frame with required timeslot size and queue size. In Data Sub-Frame (DSF) period all the wireless nodes, which previously were able to allocate the timeslots, starts to transmit data in sequence (similarity to HCCA). However, authors mention that station that reserved timeslot do not need to contend for timeslot until next CSF period. The station which reserved the slot would have to wait until all the next stations in the scheduler will finish transmission. This would incur with instable jitter.

Moreover, in highly interfered environment, which is typical nowadays there might be a starvation for some of the wireless stations, since they wouldn't be able to successfully reserve DSF period timeslots due to collisions. Similar approach was made by An et al. (2010) which again proposed two phases: DCF and TDMA. The same approach was mentioned, that during DCF period STAs transmit a request frame to AP, which records the MAC address of STA and assigns a slot in scheduler.

Other authors Tuysuz et. al (2013) tried to expose exponential back off counter and thus they called it a pseudo TDMA. The key moment is that upon successful transmission station freezes the back off counter and uses that value for next transmission attempt. This is done on all stations, which are trying to contend for wireless medium. The beacon here is used to inform station about available slots. Initial back off counter for all newly connected stations is set as 16, if station detects that there is available free slot between those occupied slots it starts to transmit its data and thus reserve that slot. However, there will be a situation that in the same channel we have EDCA based device, there won't be enough slots to assign to different stations and therefore BCCA stations will have a starvation problem. Therefore, it can be assumed, that maximum number of supported stations is equal to 16 as stated in their simulations.

In (Ting et al. 2006; Ting et al. 2007) authors propose a grouping mechanism, which would create certain groups where only selected stations can compete for wireless medium. The broadcast beacon frame which in TIM field includes information about selected stations, however there are no details how often and based on what are those transmissions handled. Authors name the proposal as TDMA, however, group contains at least 2 stations, which are using standard based wireless medium approach, thus all the contention based mechanism is handled by 
EDCA. Therefore, it might be that more active stations can take more airtime decrease group's maximum capacity, especially in those cases when a high difference between station's data rates occurs. The estimated capacity of proposal was compared to DCF based on Bianchi model (Bianchi 2000) together with simulation results, from which they have a 79\% PHY rate efficiency, when rates are $216 \mathrm{Mbps}$ for stations, 49\% PHY rate efficiency, when rates are $864 \mathrm{Mbps}$. So maximum expected throughput per AP would be $170 \mathrm{Mbps}$ and $423 \mathrm{Mbps}$, the given capacities is nowhere near the optimal ones, which should be expected. Expected capacity by using PCF, would be near $85 \%$ assuming perfect channel conditions, considering both PHY Rates. Therefore, this attempt of MAC enhancement is clearly not well suited for scaling.

Hsieh et al. (2015) propose a combination of contention based mechanism and TDMA. CSMA was used between interconnection of APs, while TDMA approach was used for communication with STAs. Since, this is a sensor wireless network topology. TDMA was implemented with accordance of wakeup/sleep cycles. During idle period STAs would be moved to power saving mode (transceiver is turned off). While on active cycle, STAs would again turn of and transmit in its assigned slot. To be more precise, each STA device had an synchronized timer on BSS and transmitted only on its assigned slot. Since, it's a Wireless Sensor Network (WSN) typically STAs has only small amounts of data to transmit, therefore slot sizes can be as low as $1 \mathrm{~ms}$ per slot.

Shoaei et al. (2015) propose a hybrid approach of TDMA and EDCA protocols. The TDMA is used for more active clients while Contention Period phase is used for less active clients. The difference between all the rest is that it does not use CSMA to send request frames for TDMA slot allocation. CSMA is used for data frames transmissions in the same way as TDMA, however it's based on contention and with idea that there won't be high number of active CPEs. The slot allocation is based on learning mechanism and scheduling problem is solved by using infinite horizon non-Bayesian multi-armed bandit (MAB). A structure consists of standard TDMA features like beacon, super frame consisting both of TDMA and CSMA phases. TDMA is split into multiple slots for active clients, while CSMA is used for non-active clients. The phase size is resolved by using $\mathrm{MAB}$ and evaluating historical user traffic profiles. Therefore, a main contribution of this paper is a scheduler design when traffic statistics are unknown. This is released together with reward system; thus authors try to solve optimization problem in order to increase total system utilization based on traffic patterns.

There have been several studies of the effectiveness of CSMA and TDMA operation under different scenarios (Munari et al. 2011). The results showed, that TDMA approach is much more effective in cooperation scenario. TDMA can achieve almost double the throughput in the same application scenarios as compared to CSMA (Munari et al. 2011). Instead of running a distributed manner 
protocol like EDCA, authors used TDMA in frequency re-use type of method, where individual master and slaves in the same cluster were able to synchronize their transmission and reception at the same time, thus increasing overall system capacity. Furthermore, authors discuss that at light network load, CSMA might provide some advantages when comparing to TDMA approach. In TDMA interval between retransmissions might be shorter when comparing to EDCA. This is because TDMA uses higher rates when compared to EDCA retransmissions. Therefore, EDCA wireless nodes with excessive retransmissions might gain better performance in unfavorable positions. However, this was not researched more deeply by the authors since such advantage does not emerge in considered TDMA implementations, because either small packets are delivered at first attempt or dropped, which is not the case in real world applications.

The TDMA by Lin et al. (2014) is similar to all the rest approaches before. The idea is established by using TDMA as replacement for CFP of 802.11e and DCF for low priority traffic. The approach is selected to increase the probability of packets delivery. From the results, the approach provides $20 \%$ higher packet delivery ratio. Similar approaches are provided in another articles (Liaw, Yeh 2011; Jun et al. 2006). Like in all the rest articles timeslots are carried by the beacon, after which individual slots are assigned to stations. Thus, stations start the transmission only on assigned slot. This is a simple round robin based implementation as compared to more advanced techniques. In order to keep compatibility with already existing wireless devices, however the method was selected to leave some part of time available for contention period.

Similar approach is taken by Li et al. (2007) and Li et al. (2006) the improvement here is a real time allocation of transmission slots based on throughput requirements, so time slots are assigned more flexible. The RTS/CTS here is exposed as a handshake mechanism to complete the slot assignment for any newly connected station. Since this scheme is applied to Ad Hoc network it can assign time slots effectively by coordinating announcements and confirmations with neighbor nodes. From the provided results seems this more appropriate for indoor based wireless sensor networks and not reliable for outdoor backhaul or point to multipoint communication (last mile connectivity). Khan et al. (2015) proposed a different TDMA approach. Particularly, it's background is based on pseudo-time division multiple access (PTDMA). In PTDMA each active node starts with EDCA based wireless medium access mechanism. After successful transmission wireless node switches to a pseudo periodic transmission similar to TDMA, if any collision happens and they usually will happen, wireless node will switch back to EDCA. Therefore, such method has a drawback of being inflexible for heterogeneous traffic. It is mentioned that PTDMA improves effective capacity when compared to EDCA in saturated networks. However, it's quite opposite when the network is unsaturated. In this case EDCA provides better performance due to its 
flexibility and performs well for bursty heterogeneous traffic scenarios, however it suffers from performance degradation under heavy contention and traffic resulting from large number of collisions (Choi et. al 2005).

Since PTDMA has fixed timeslots, authors propose an alternative to PTDMA Self-Organized TDMA (SO-TDMA). When network is saturated, operation is identical to PTDMA, however when network is not saturated it uses a flexible method to adapt timeslot to throughput requirement. Since slot size is flexible, efficiency increases when traffic flow is low. The important parameter of PTDMA is transmission time-slot size, which directly determines the performance of protocol in various cases. If the value is too low channel utilization will be too low. If the value is too high, the delay will increase between transmissions. Similarly, the transmission queue length has to control as well, so there wouldn't be any gaps left between transmissions. SO-TDMA encompasses dynamic transmission slot length and that's the main improvement for capacity when comparing to a fixed time slot PTDMA approach.

Classic TDMA approach was used to improve voice quality (Guo et al. 2007). Authors divided available transmission time into several slots and allocated to connected stations. At the start of TDMA super frame, there's a broadcast frame with allocated slots and its duration. The proposed TDMA consists of $20 \mathrm{~ms}$ schedule cycles. Each schedule is broken down into broadcast period, voice period and data period. So a broadcast period contains information about available and occupied timeslots. Voice period, which is used as a group of timeslot for station to send voice packets, a single timeslot for downlink voice from AP (statistical multiplexing by using single slot to transmit all downlink packets is used). Finally, a data period, which consists of multiple data slots for uplink and at the end single downlink timeslot for AP. Additionally, there is a contention slot, that is being used for non-active stations data transmission. However, if any of these slots would be interrupted by outside interference source, it's likely system would have a high packet loss.

\subsubsection{State-of-the-art Wireless Medium Access}

In these days, State-of-the-art devices that are provided for Wireless Internet Service Providers rarely employs the rules defined by the standard. Most of the Stateof-the-art devices implements their own modification of IEEE 802.11 WM access schemes or completely rebuilds the WM scheme such as TDMA approach. To fully compare the suggested solution, it's needed to understand the limits and operation basics of the State-of-the-art implementation, that are usually closed source and requires additional packet captures and observation to fully analyze the wireless medium access approaches being used. 
Time Division Multiple Access allows to disable the standard IEEE 802.11 listen before talk mechanism, which is used in all off-the-shelf devices these days. Instead of providing distributed access, TDMA provides a timeslot for each connected station. The fixed timeslot prevents station from occupying wireless medium excessively. Control of transmission time is rather convenient way to solve the network efficiency issues when system has several stations with bad connectivity. In example, low signal levels and low data rates causes system performance degradation. The low modulations results in higher airtime occupation time, which is needed for data frames transmission (Natkaniec et al. 2015).

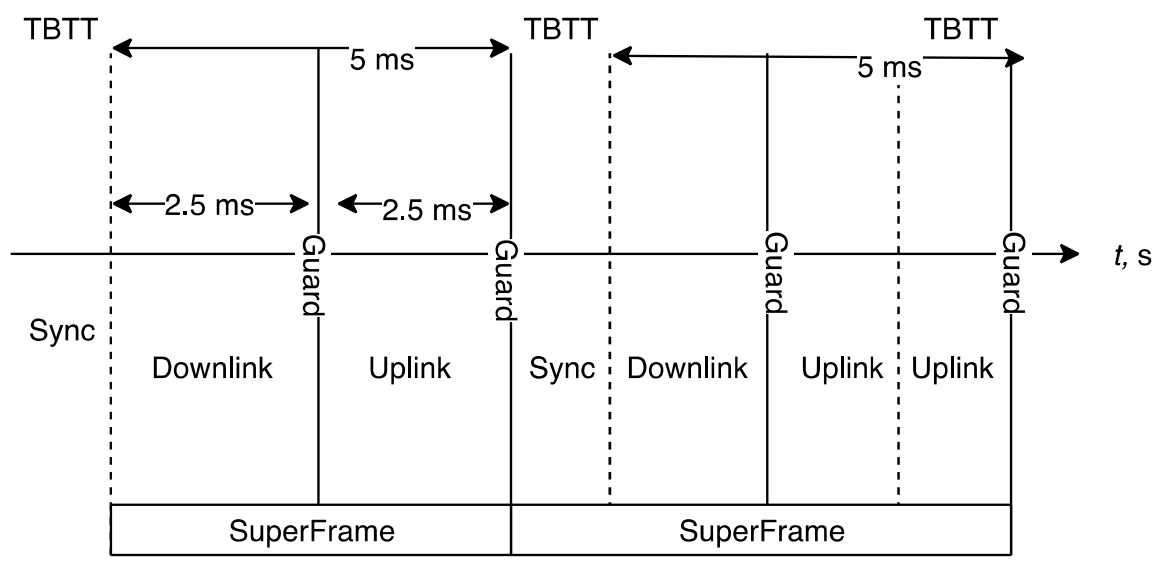

Fig. 2.5. Time Division Multiple Access super frame structure

However, TDMA at the same time introduces additional management frames as well as increased latency, since next transmit attempt can happen only at the predefined timeslot for connected station, therefore it's not always acceptable for end applications like voice service. This can be solved by leveraging timeslot size, however lower timeslot size results in lower throughput, but provides lower latency, which is needed for voice service, this is the tradeoff between delay and throughput. Leffler (2009) states that TDMA system which is software based will be less efficient than the TDMA systems based on the hardware assistance, therefore most of the times proprietary TDMA systems in the off-the-shelf devices uses the IEEE 802.11 defined features such as TBTT. The TDMA has a master node, Access Point, that handles the connectivity and WM access control. The AP broadcasts beacon frames, that include the connection information and availability of time slots. Finally, the same beacon frames provide synchronization for the connected stations, so stations could adjust the timers. When the station connects to such AP, it starts to generate a management frames, which both works as a 
keep-alive messages in order to keep the link active and reserve the transmission timeslot. In (Leffler 2009) it is defined that granularity of beacon time has a $1 \mathrm{TU}$ $(1024 \mu \mathrm{s})$ granularity, which is enough for building on existing IEEE $802.11 \mathrm{de}-$ vice, the proprietary TDMA system. The beacon transmission process is done by the IEEE 802.11 hardware and happens periodically during the super frame, same approach is done in the proprietary TDMA system, the only difference is the period when these frames are being transmitted. Each implementation for different wireless vendor might have its own period for synchronization frames. Leffler (2009) defines that synchronization happens after the station receives beacon frame, before pushing the packet to the host, the local TSF snapshot is provided from hardware, that can be used to find out the start of the frame by adjusting the value based on the air time required for transmission.

The adjusted value is compared to the local hardware timers and any required synchronization changes are performed instantly. Leffler (2009) explanation is based on the old IEEE 802.11 standard, the same approach is being performed in the most recent IEEE 802.11ac off-the-shelf devices, the only thing which differs is how the WM is being handled and the place where the timers are being adjusted. Since the most recent IEEE 802.11ac implementations has a split architecture, where transmission and WM is handled in offload subsystem and all the connectivity and other sub processing is being done in the host side.

More advanced systems uses a built-in Global Positioning System (GPS) module to adjust timers between connected stations and the AP and this is primarily used in State-of-the-art systems these days. The vendors which provide TDMA access in unlicensed band usually build a base station with GPS module included for better time granularity due to higher time drift probability when using the beacon frame based synchronization. Additionally, the TDMA has to control and prevent any station from exceeding the transmission timeslot for this reason additional guard interval between downlink and uplink slots is added. TDMA has a super frame timeslot, which defines both uplink and downlink timeslots for transmission, each super frame timeslot is separated by guard interval. The guard interval usually serves as a precaution against packet collisions. The super frame structure in Fig. 2.5. indicates both uplink and downlink timeslots that are defined in $5 \mathrm{~ms}$ super frame. The interval is split even by $2.5 \mathrm{~ms}$ for uplink and downlink. However, the more advanced approach can split the $5 \mathrm{~ms}$ super frame into several additional sub-timeslots, when several stations requires uplink time, but has very little data to send. Therefore, the timeslot length for downlink and uplink can be variable depending on the stations activity.

The Fig. 2.6. shows the basic concept of State-of-the-art TDMA approach, which is being used by one of the wireless equipment vendor. The scheme is taken based on the actual packet capture and evaluation under different terms, with and without traffic load and variable number of stations on AP. Thus, a closed source 
protocol is being analyzed to understand the actual implementation of wireless medium access in State-of-the-art devices. In following scheme, the sync frame is mainly used for keeping stations and AP transmission timers synchronized in order to avoid collisions, while Guard Interval is usually fixed based on the distance between AP and STA. The typical implementation of TDMA in State-of-the-Art devices includes the Sync frames and Acknowledgement frames. Depending on the situation there might be several additional management frames, which indicates the start and the stop of the station timeslot. The start and stop frames, includes the NAV field, which is set $32,766 \mathrm{~ms}$. The NAV duration field is the maximum allowed NAV field by IEEE 802.11 specification and isn't used under any normal conditions in today's wireless networks, that operate under IEEE 802.11 EDCA approach.

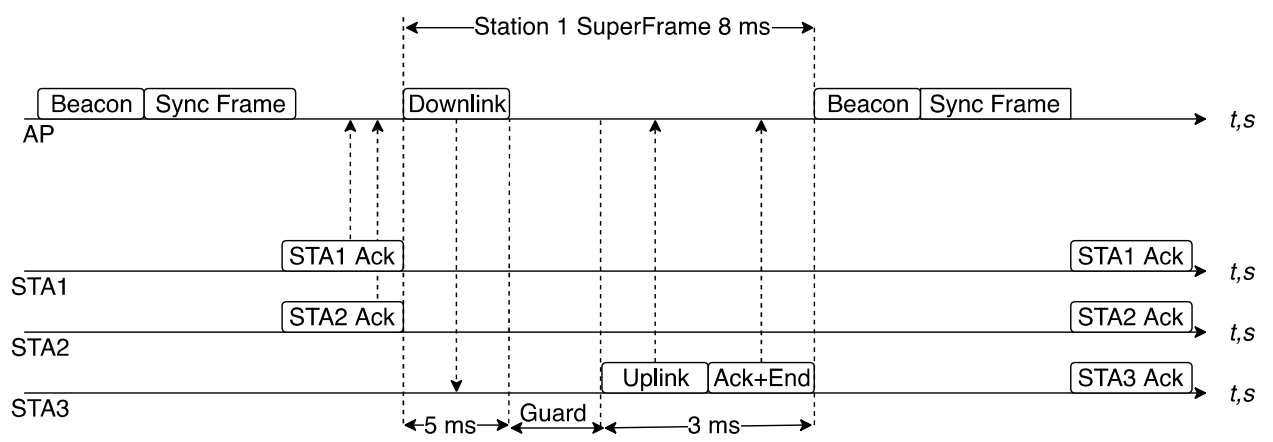

Fig. 2.6. Proprietary Time Division Multiple Access scheme

This is similar approach to PCF, it reserves airtime for specific period, however TDMA reserves channel medium completely from any other operational devices. Thus, TDMA operating on the channel prevents standard IEEE 802.11 devices from wireless medium access. This is done based on the fact that standard IEEE 802.11 EDCA devices uses NAV as indication for channel occupancy (the virtual carrier sense), due to constant back off mechanism operation standard IEEE 802.11 EDCA devices can't gain access to the wireless medium. Since every transmission start with management frame that sets NAV, therefore all stations, that are non-TDMA won't be able to use the WM. The field is either updated by hardware based on the transmitted packet length and the selected transmission rate or is used static as in TDMA. Therefore, duty cycle in channel where TDMA devices operates, takes up to $90 \%$ and prevents any other AP operation (Szott et al. 2014; Nupur et. al 2011) as in Fig. 2.7.

In order to decrease RTT in point to multipoint networks TDMA has to implement sub-timeslots, that are used for less active stations. However, such change 
is a tradeoff between throughput and round-trip-time. The more sub-timeslots are used to put transmission of stations the lower round trip time will be, however in exchange throughput would be much lower. Usually in wireless networks traffic is asymmetric, where Downlink takes larger half of transmission time and Uplink is used just for Acknowledging frames.

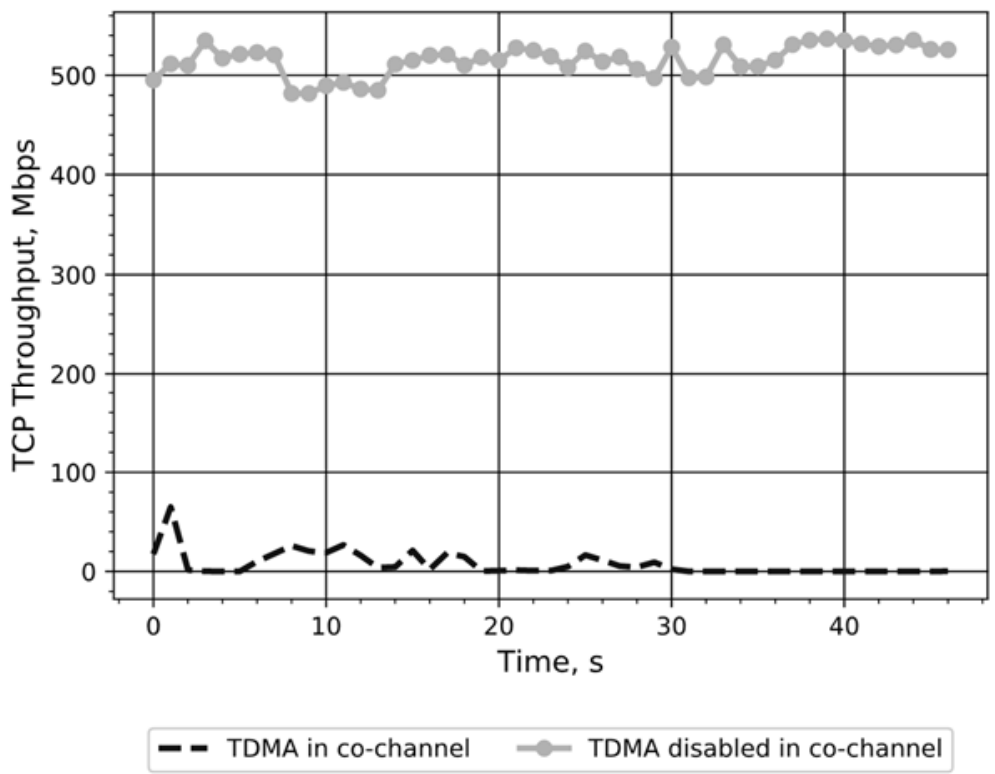

Fig. 2.7. Time Division Multiple Access duty cycle in operational channel

In the Fig. 2.8 an actual proprietary TDMA packet capture is displayed. In this capture an $8 \mathrm{~ms}$ super frame is indicated by the time field column. The super frame, downlink is split into several sub-timeslots, for each individual station downlink. The station which didn't receive the required sub-timeslot, sends the ack at the very beginning of the transmission, while the other 3 starts to transmit data. The last station, which sends data also sends a management frame with indication of super frame end. The sequence of transmission steps can be concluded:

1. Transmission of Sync Frame. All the connected stations receive the Sync frame to update their timers.

2. The stations which aren't sending data are sending single management frame.

3. The AP starts transmitting data packets for the stations which received their predefined timeslot or sub-timeslot. The same timeslot can be defined for downlink traffic and for uplink traffic. 
4. The retries of non-received packets are retransmitted in the next timeslot cycle.

\begin{tabular}{|c|c|c|c|c|c|c|c|}
\hline No. & Time & Destination & Protocol & Length & Data Rate & CTS_RTS & Duration \\
\hline 80 & 0.005 & Broadcast & 802.11 & 161 & 12.0 & Aruba Management & 30741 \\
\hline 84 & 0.009 & Ubiquiti... & 802.11 & 80 & 12.0 & Aruba Management & 32764 \\
\hline 93 & 0.010 & Ubiquiti... & 802.11 & 80 & 12.0 & Aruba Management & 32766 \\
\hline 94 & 0.010 & Ubiquiti... & 802.11 & 80 & 12.0 & Aruba Management & 32763 \\
\hline 95 & 0.011 & Broadcast & 802.11 & 153 & 12.0 & Aruba Management & 30741 \\
\hline 138 & 0.019 & Broadcast & 802.11 & 161 & 12.0 & Aruba Management & 30741 \\
\hline 147 & 0.025 & Ubiquiti... & 802.11 & 80 & 12.0 & Aruba Management & 32764 \\
\hline 148 & 0.025 & Ubiquiti... & 802.11 & 80 & 12.0 & Aruba Management & 32766 \\
\hline 210 & 0.030 & Broadcast & 802.11 & 153 & 12.0 & Aruba Management & 30741 \\
\hline 282 & 0.037 & Ubiquiti... & 802.11 & 70 & 12.0 & Aruba Management & 32764 \\
\hline 283 & 0.037 & Broadcast & 802.11 & 165 & 12.0 & Aruba Management & 30741 \\
\hline 287 & 0.041 & Ubiquiti... & 802.11 & 80 & 12.0 & Aruba Management & 32763 \\
\hline 288 & 0.041 & Ubiquiti... & 802.11 & 80 & 12.0 & Aruba Management & 32765 \\
\hline 289 & 0.041 & Ubiquiti... & 802.11 & 80 & 12.0 & Aruba Management & 32764 \\
\hline 294 & 0.042 & Ubiquiti... & 802.11 & 70 & 12.0 & Aruba Management & 32766 \\
\hline 295 & 0.043 & Broadcast & 802.11 & 153 & 12.0 & Aruba Management & 30741 \\
\hline 341 & 0.051 & Broadcast & 802.11 & 161 & 12.0 & Aruba Management & 30741 \\
\hline 344 & 0.057 & Ubiquiti... & 802.11 & 80 & 12.0 & Aruba Management & 32764 \\
\hline 345 & 0.057 & Ubiquiti... & 802.11 & 80 & 12.0 & Aruba Management & 32766 \\
\hline 410 & 0.062 & Broadcast & 802.11 & 153 & 12.0 & Aruba Management & 30741 \\
\hline 481 & 0.070 & Ubiquiti... & 802.11 & 70 & 12.0 & Aruba Management & 32764 \\
\hline 482 & 0.070 & Broadcast & 802.11 & 165 & 12.0 & Aruba Management & 30741 \\
\hline 485 & 0.073 & Ubiquiti... & 802.11 & 80 & 12.0 & Aruba Management & 32763 \\
\hline 486 & 0.073 & Ubiquiti... & 802.11 & 80 & 12.0 & Aruba Management & 32765 \\
\hline 487 & 0.073 & Ubiquiti... & 802.11 & 80 & 12.0 & Aruba Management & 32764 \\
\hline 492 & 0.074 & Ubiquiti... & 802.11 & 70 & 12.0 & Aruba Management & 32766 \\
\hline
\end{tabular}

Fig. 2.8. Wireshark Capture of IEEE 802.11 Time Division Multiple Access proprietary implementation

In order that TDMA would work it has to ignore any other activity in the channel, since any backoff timer would interrupt the timing and would result in the collisions. The NAV field which is set during broadcast and sync management frames sets the wireless duration field as in Fig. 2.9. The NAV field is either updated by hardware based on the transmitted packet length and the selected transmission rate or is used static. In this case TDMA uses a static NAV duration field.

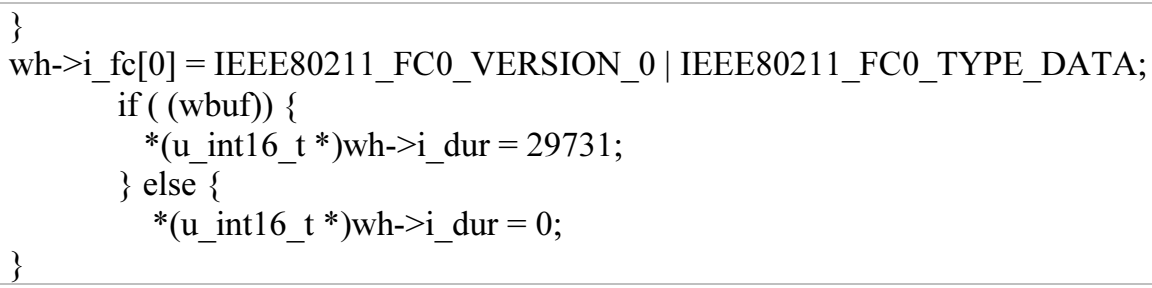

Fig. 2.9. Wireless duration field update 
Another feature of TDMA is that timeslots can be divided by downlink and uplink throughput ratio: $75 / 25,50 / 50$. The ratios indicates the time for downlink and the time for uplink. In dense wireless networks the usual preference by stateof-the-art devices is $75 / 25$, since mostly the downlink traffic is being used in these days. State-of-the-art TDMA approach introduces additional concept, that can't be seen in traditional wireless medium access mechanisms or the research articles. Frequency re-use capability, the possibility to re-use same frequency on tower on multiple Master APs. This increases spectrum utilization efficiency. Traditional approaches would either cause out of band interference due to adjacent channel interference, which has been studied or cause unfair wireless medium sharing between multiple APs operating on same frequency.

Therefore, TDMA system is able to resolve the above mentioned problems. The key moment here on traditional systems is that one of the APs might be receiving frames, while another will start transmission and thus this creates collisions and drastic performance decrease. This is especially noticeable in hidden wireless node topologies (Zhong et al. 2015). The TDMA multi sector synchronization is done by syncing Tx and Rx of multiple sectors located on the same tower. However, even TDMA requires a proper allocation of transmit power in order to prevent collisions between remote sites.

\subsection{A Novel IEEE 802.11n Multi-Poll Protocol Implementation}

The proposed novel Multi-Poll protocol is based on off-the-shelf device functionality and tries to re-use as many functionality from hardware accelerator based functions as possible with the aim to keep compatibility with legacy devices operating on the same channel. The actual modification of proposed Multi-Poll protocol re-uses both the hardware based retry mechanism as well as Qualcomm's (Qualcomm 2018) built-in functionality for throughput enhancement by allowing to burst several frames with SIFS between them as long as the frames are from the same Access Category. Such option as in Fig. 2.10. allows to burst more packets on single access to WM. Maximum of 12 AMPDU can burst on single access with $1024 \mu$ s limit per AMPDU.

Under low traffic conditions Multi-Poll protocol operates under EDCA rules. Meaning that each device operating in the BSS listens before transmitting any data. This includes both the usage of back off and AIFS to gain access to WM. It has to be noted that the operational system uses above mentioned SIFS bursting implementation for all ACs.

The next phase happens when one of the stations matches the defined traffic pattern thresholds or goes above them for longer than the defined period. In such 
case the initial transmission still happens under EDCA rules, but after first detection of such activity AP initiates an active polling stage. The poll frame is not sent at the lowest data rate as typically happens for control frames in IEEE 802.11 standard. Instead it is sent at the highest possible data rate, that can be used for the current station. This approach allows to increase efficiency as well as decreases the actual airtime needed for the management frames that controls the wireless medium.

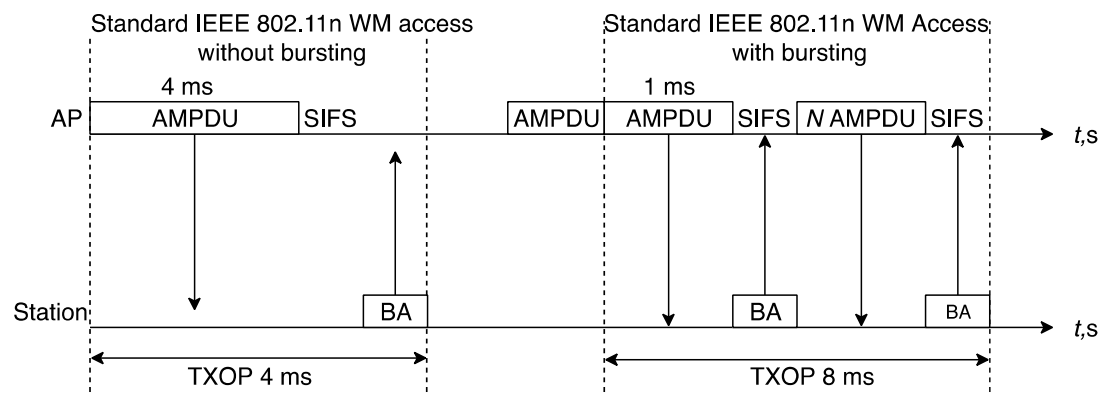

Fig. 2.10. Short Inter Frame Spacing in IEEE 802.11 wireless medium access

The IEEE 802.11n defines three types of preamble (IEEE Std 802.11-2016), see Fig. 2.11. The High Throughput Mixed Mode Format (HT MF) preamble is used in order to support legacy devices, by including Legacy Short Training Field (L-STF), Legacy Long Training Field (L-LTF), Legacy Signaling Field (L-SIG), High Throughput Signal Field (HT-SIG1), HT-SIG2, High Throughput Short Signal Field (HT-STF), High Throughput Long Signal Field (HT-LTF1) up to HT(LTFN), the service field which is equal to 16 and the actual High Throughput Data (HT-DATA) with additional tail and padding if required.

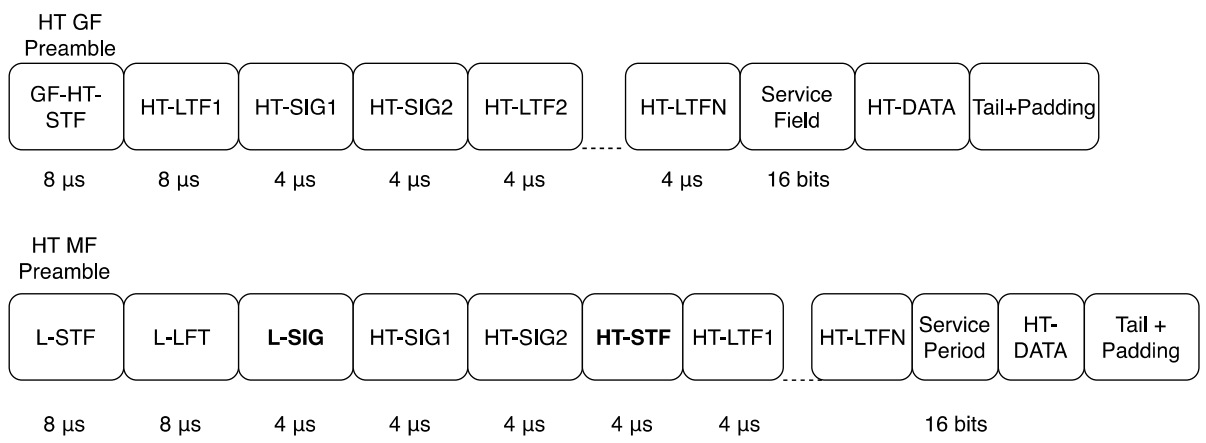

Fig. 2.11. Greenfield and High throughput mixed mode Preamble format (IEEE Std 802.11-2016) 
The deployments, which do not need legacy devices support, are called Greenfield deployments. By disabling legacy device support, the preamble removes the legacy fields. Instead preamble has an Greenfield High Throughput Short Training Field (GF-HT-STF) Same as HT-Mixed Mode, GF mode has to perform the same CCA procedures based on the TXTIME calculated from the contents of signal field (Perahia, Stacey 2013). The TXTIME equation for GF is expressed below:

$$
T X T I M E=T_{\mathrm{GF}-\mathrm{HT}-\mathrm{STF}}+T_{\mathrm{HT}-\mathrm{LTF} 1}+T_{\mathrm{HT}-\mathrm{SIG}}+\left(N_{\mathrm{LTF}}-1\right) \cdot T_{\mathrm{HT}-\mathrm{LTFs}}+T_{\mathrm{SYM}} \cdot N_{\mathrm{SYM}},
$$

where $T_{\mathrm{GF}-\mathrm{HT}-\mathrm{STF}}, T_{\mathrm{HT}-\mathrm{LTF} 1}, T_{\mathrm{HT}-\mathrm{SIG}}=8 \mu$ duration; $T_{\mathrm{HT}-\mathrm{LTFs}}=4 \mu \mathrm{s} ; T_{\mathrm{SYM}}=4 \mu \mathrm{s}$ for $800 \mathrm{~ns}$ guard interval or $3.6 \mu$ s for $400 \mathrm{~ns}$ guard interval. $N_{\mathrm{SYM}}$ in the TXTIME is calculated based on the following expression (Perahia, Stacey 2013):

$$
N_{\mathrm{SYM}}=\left[\frac{8 \times A M P D U+16}{N_{\mathrm{DBPS}}}\right],
$$

where $A M P D U$ is the length of the data packets and $N_{\text {DBPS }}$ is the number of data bits per OFDM symbol as is taken from the Table 2.1.

Table 2.1. Modulation Coding Scheme parameters for optional $40 \mathrm{MHz} N_{S S}=2, N_{E S}=1$ (IEEE Std 802.11-2016)

\begin{tabular}{|c|c|c|c|c|}
\hline \multirow{2}{*}{ MCS index } & \multirow{2}{*}{ Modulation } & \multicolumn{2}{l|}{$N_{\text {DBPS }}$} & \multicolumn{2}{|l|}{ Data rate, Mbps } \\
\cline { 3 - 5 } & & & $800 \mathrm{~ns}$ GI & 400 ns GI \\
\hline 8 & BPSK & 108 & 27.0 & 30.0 \\
\hline 9 & QPSK & 216 & 54.0 & 60.0 \\
\hline 10 & QPSK & 324 & 81.0 & 90.0 \\
\hline 11 & 16-QAM & 432 & 108.0 & 120.0 \\
\hline 12 & 16-QAM & 648 & 162.0 & 180.0 \\
\hline 13 & 64-QAM & 864 & 216.0 & 240.0 \\
\hline 14 & 64-QAM & 972 & 243.0 & 270.0 \\
\hline 15 & 64-QAM & 1080 & 270.0 & 300.0 \\
\hline
\end{tabular}

Table 2.2. $N_{\text {SYM }}$ with different Modulation Coding Scheme

\begin{tabular}{|c|c|c|}
\hline $\begin{array}{c}\text { A_MPDU_LENGTH, } \\
\text { bytes }\end{array}$ & $\begin{array}{c}\text { MCS0, } \\
N_{\text {SYM }}\end{array}$ & MCS15, $N_{\text {SYM }}$ \\
\hline 116 & 9 & 1 \\
\hline
\end{tabular}


Based on the TXTIME formula before, the result for $N_{\text {SYM }}$ with different MCS9 and MCS1 is shown in Table 2.2. Based on the Table 2.2 data an airtime occupation was calculated when different MCS are being used. The MCS0 is used for all management frames both in EDCA and PCF based approaches (IEEE Std 802.112016) and MCS15 which is used only for control polling frames in Multi-Poll based approach. The representation of different airtime usage for polling frames when different MCS is being used can be seen in Fig 2.12, the Multi-Poll based airtime management frames generates up to 10 times lower airtime usage when compared to standard PCF based approach used polling frames and assuming there is more than 1500 token frames. The graph is assuming that Greenfield preamble is being used and network consists of more than 30 active stations.

Another improvement is that Multi-Poll active stations are only receiving polling frames if these stations are in the active list. The inactive stations do not require any polling frames and simply operates based on EDCA rules Fig. 2.13. Therefore, an active station can't send any data without being polled. The similar idea is defined in Cheng, $\mathrm{Wu}$ (2009) authors propose to have two stages, when stations receive an ACK frame which indicates that station is being polled and station enters Contention Free mode in which case transmission happens after Contention Inter-frame Space which is lower than DIFS typically used by EDCA. In case station does not have any data to send it stays silent and all the rest stations operates under EDCA rules.

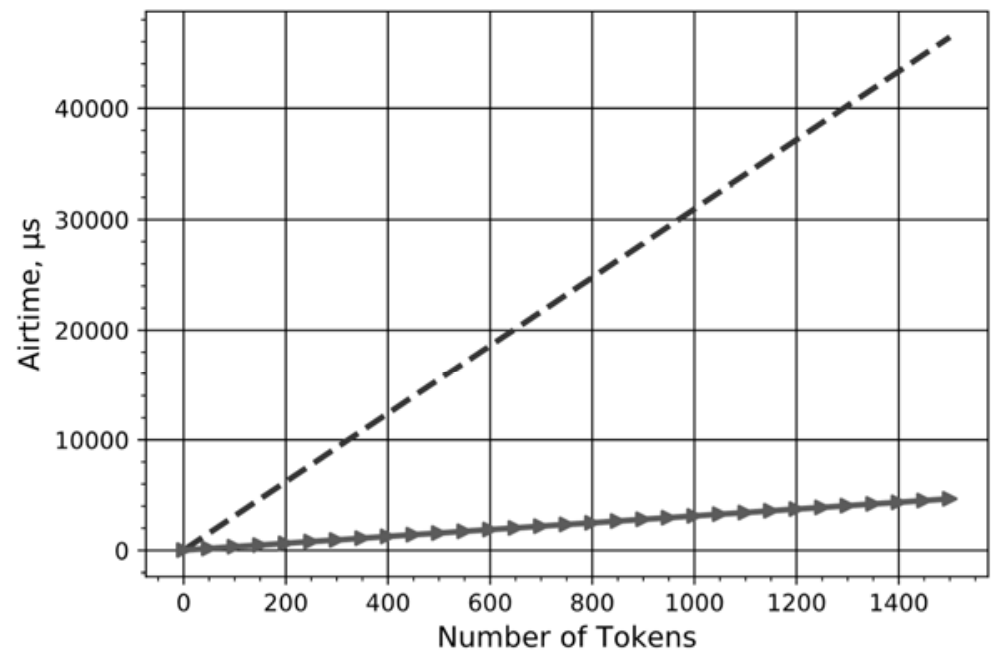

IEEE 802.11 GF Token Airtime with MCS0 IEEE 802.11 GF Token Airtime with MCS15

Fig. 2.12. Airtime usage as a function of number of tokens 
However, such implementation requires more complex modifications for the standard IEEE 802.11 protocol as well as does not take into account different ACs that builds IEEE 802.11 QoS functionality. The appearance of AIFS in IEEE 802.11e provides ability for AP to reserve wireless medium faster due to different $\mathrm{CW}$ and AIFS parameters across the BSS. In this case AP and station are using different $\mathrm{CW}$ and AIFS, TxOp values. Additionally, the implementation provided by $\mathrm{Chen}, \mathrm{Wu}(2009)$ suggests that it would be constantly switching back and forth between modes active and idle states due to the reason that typical IEEE 802.11 wireless networks handle quite a lot of broadcast packets which would trigger state change for every connected station and therefore such implementation would be constantly operating under polling based rules.

The proposed novel Multi-Poll implementation is based on the fact that nowadays produced wireless IEEE 802.11 devices can handle the collisions due to the more advanced rate control algorithms, which are defined in the next chapter.

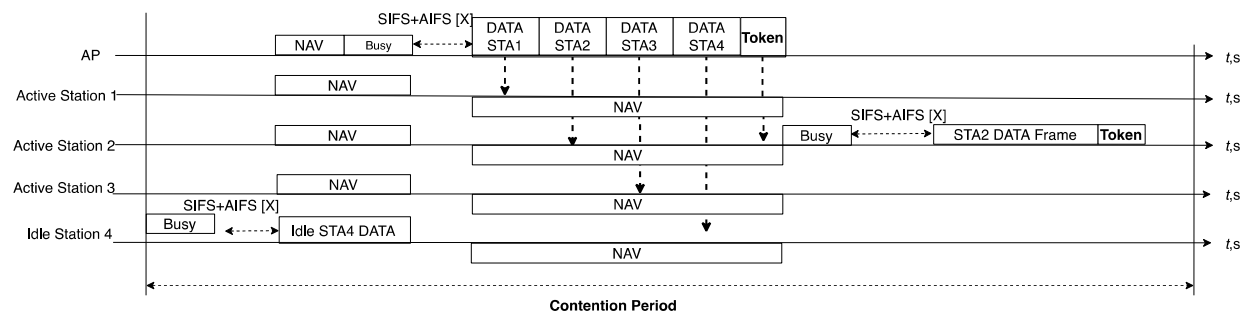

Fig. 2.13. Multi-Poll wireless medium access scheme during idle period

The actual number how many collisions can be handled between hidden stations was not researched so far in high density network deployments and identification of possibility to allow certain number of collisions is a key moment in building a novel and more advanced active, idling stations scheduler mechanism.

The off-the-shelf rate control algorithm modification allows to handle collisions in such cases when system has a hidden stations deployed. This is due to the fact that rate control algorithm tries to repeat the same frame 4 times and later executes RTS/CTS to reserve airtime, in case this does not help it tries to lower down the modulation (the rate series table) and repeats the same steps again until it receives ACK that packets were received or simply drops them. However, by using a combination of RSSI based rate control on stations and PER based rate control on AP, a system can handle the increasing number of collisions without decreasing the actual data rate, which would happen when PER based rate control algorithm is used. The general rules of transmission is such that depending on station activity the AP handles it's transmission as can be seen from Fig 2.14. Under normal conditions when throughput is below defined thresholds all stations 
are operating under EDCA, where they gain access to WM by competing according to IEEE 802.11e operational rules. Each access category has its own AIFS period after which station can compete for the wireless medium, if it was idle for AIFS period during listening period. When the throughput reaches the defined limits or surpasses it, station receives a poll frame from the AP and later the access to WM can happen only if station receives poll frames from the AP.

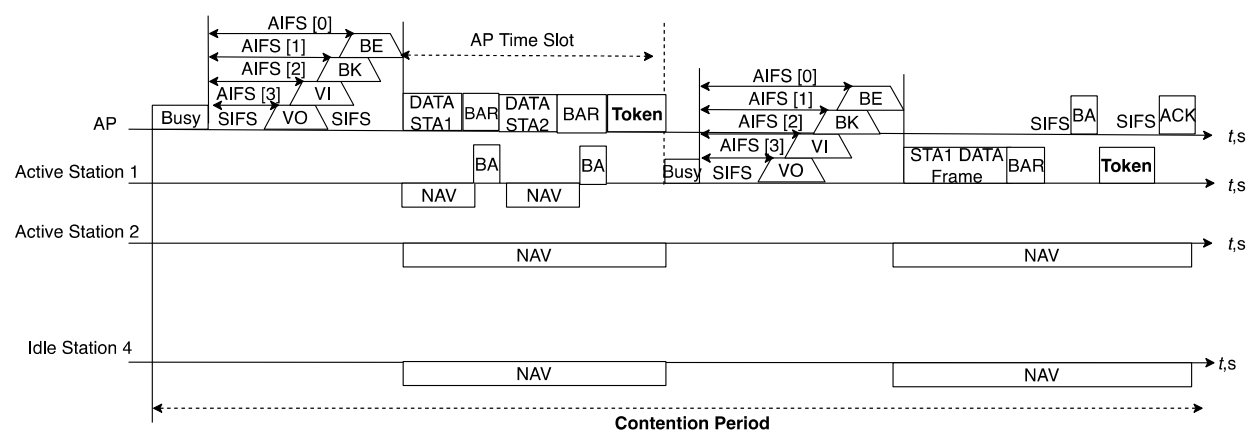

Fig. 2.14. Multi-Poll wireless medium access scheme during active period

The poll frames are required only for stations which throughput or packet per second values are above the defined minimum threshold values. The latter scheme, above has a similarity to IEEE 802.11 ax wireless medium operation (Bankov et al. 2017). The IEEE 802.11 ax uses a group randomly addressed polling (GRAP), therefore each station receives a polling frame. GRAP allows a backwards compatibility with already existing protocols with possibility to perform multiple access polling technique (Bellalta et al. 2017; IEEE Std 802.11-2016). In IEEE 802.11ax the AP contends for wireless medium by using and Best Effort access queue. The trigger frame (polling frame) is being send to every connected station. The use of trigger frame is to allocate resource unit for each of the stations. In case station does not have enough data to send, it simply does not start transmission. The IEEE 802.11ax defines that only APs are contending and stations are transmitting on assigned slot.

The AP gains access by performing contention based access and the beacon frame has a NAV field set in order to prevent legacy devices communication. When the medium is clear for PIFS duration the AP issues a polling frame to all the connected stations. After that each station can perform data transmission or do not transmit anything, since IEEE 802.11 ax adds new physical layer it allocates sub-carriers for the stations, which will be transmitting data. Up to 8 clients can communicate simultaneously. Each station can transmit only on allocation radio blocks, the downlink and uplink is divided by transmitting CF-poll frames. The end of CFP period provides possibility for legacy stations communication, that 
use standard contention based access. Important detail to IEEE 802.11ax wireless medium access is that stations do not perform any CCA due to reason that performing CCA might disrupts wireless medium access timings (Zhong et al. 2016), therefore in order to have efficiency there has to be no other station operating in the same channel, otherwise there will be a high performance decrease as seen with TDMA protocol before (Afaqui et al. 2017).

Additionally, the stations operating in the same BSS has to be actively polled and limited in transmit time by the AP. Otherwise the collisions might happen, the similar approach is implemented by State-of-the-art Qualcomm implementation, where radio resources are split across connected stations and each of these stations are being triggered by active polling to assigned radio resource blocks.

When comparing the Multi-poll scheme to the HCCA operation, there is no strict CFP and CP periods, during which stations are required to update the NAV field based on AP requirement. Instead all stations are operating under EDCA conditions all the time, the only difference is that to decrease the number of collisions AP uses poll frames to split the airtime between the most active stations. Such scheme is similar to the IEEE HCCA scheme with following changes:

1. Low traffic pattern in the wireless medium results in normal IEEE 802.11e EDCA operation in all BSS

2. Stations that reach defined throughput or packet number per second thresholds are moved into active polling list and access to WM is controlled by the AP. This is similar approach to PCF, however main difference is that all the other competing stations are not required to stop their contention based access to wireless medium.

3. The stations can be moved between active and idle operational list each time station reaches the throughput thresholds and can be moved back to idle list if their throughput decreases and is lower for defined period of time.

4. Unlike the HCCA AP is not required to reserve airtime for QoS based stations, the QoS operation follows EDCA rules by using different AIFS and TxOp parameters to gain faster access to wireless medium.

The main scheme which is proposed solves following issues. The hidden wireless node problem, when the traffic is high enough between stations which is not heard by stations operating in the same BSS, AP uses poll frames to handle the airtime reservation between stations. Thus, collisions are decreased and stations are able to pass data successfully. The idle and active steering provides ability to increase and decrease polling list, which results in higher throughput. When polling list has small amount of stations the stations are being polled more frequently and parameters such as latency is decreased and throughput is increased. This provides better QoE for end customers unlike the PCF or HCCA approach. Additionally, polling list provides ability to achieve maximum throughput unlike 
the TDMA that uses fixed timeslot for each station transmission. TDMA in cases if station does not have enough data to send but still uses same size timeslot, therefore assigned timeslot is being wasted.

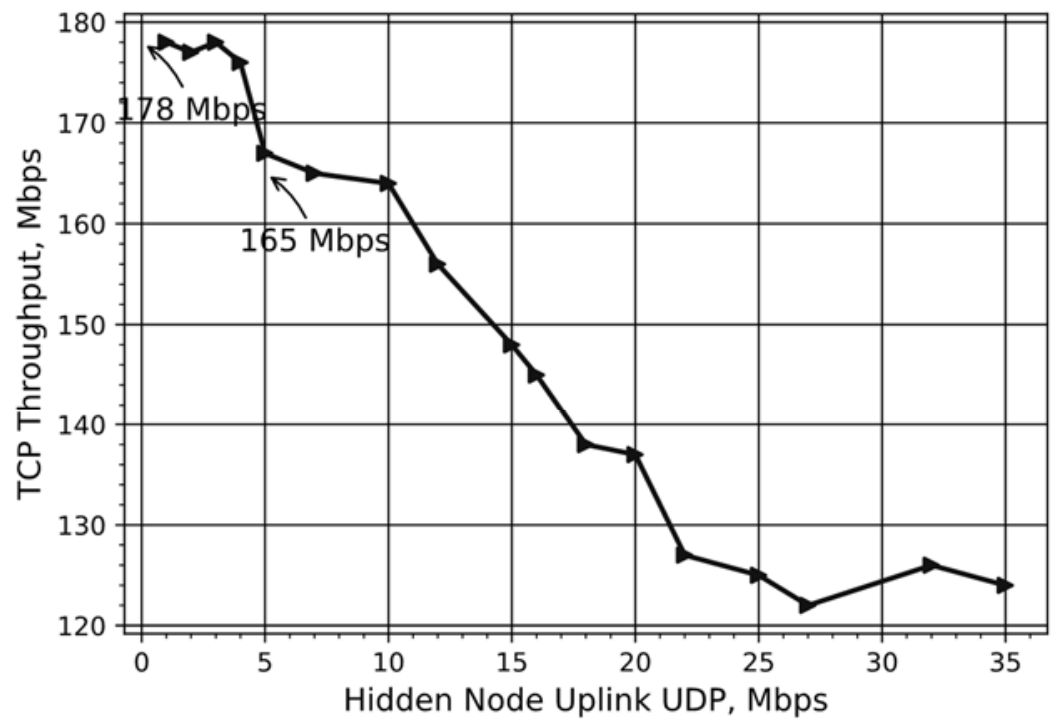

\section{$5 \mathrm{GHz}$ system performance with hidden node}

Fig. 2.15. Hidden Node Impact to System Capacity in IEEE 802.11 Network

Secondly, the active and idle steering is based on the State-of-the-art rate control algorithm operation, that allows to handle small amount of packet collisions by using RTS and CTS mechanism. Thus, when stations are allowed to compete under EDCA rules, AP is able to handle collisions up to following throughput figure that is displayed in Fig. 2.15. The graph displays that under normal operation when there is no hidden wireless node traffic present in the same BSS, the AP can reach maximum capacity of $178 \mathrm{Mbps}$ and this is a limit of the $100 \mathrm{BaseT}$ Ethernet port. However, when hidden node traffic increases it's possible to see a pattern under which the traffic decreases. It's worth to notice that this is only two active 2 stations setup out of 78 connected to the AP. First station is located $5 \mathrm{~km}$ from the AP and another is located at 200 meters. The testbed consists of Qualcomm AR9342 chipset based devices with CPU processing power $533 \mathrm{MHz}$. All, 78 devices and Access Point are based on IEEE 802.11n 2×2 MiMo with 64 QAM modulation capability. Stations, which are connected to Access Point, have respectively $15 \mathrm{dBi}$ and $20 \mathrm{dBi}$ antennas, Access Point has 90 degree $18 \mathrm{dBi}$ sector antenna. 
When stations attempt transmission it would cause collisions in standard EDCA mode. In this case a requirement is to find a value when the throughput still satisfies the performance requirements without drastically decreasing whole system capacity. As per graph the off-the-shelf based devices are still able to achieve high system capacity when continuous hidden wireless node traffic is up to $10 \%$ of total system's capacity. Therefore, the Multi-Poll assumption is that all the stations that operate under EDCA rules combined uplink traffic should not reach $10 \%$ of total system's capacity with assumption that this type of traffic is not continuous. Collisions in point to multipoint network usually happens due to uplink traffic unlike the downlink traffic therefore some of the State-of-the-art implementation sets uplink traffic trigger for each connected station to $300 \mathrm{kbps}$, however in Multi-Poll case both downlink and uplink throughput numbers are monitored in order to get faster active-idle client steering possibility.

Finally, by using EDCA based polling approach the implementation requires less changes to the off-the-shelf devices driver unlike the TDMA implementation. By using EDCA approach several APs operating in the same channel are able to access the WM, since NAV field is not set to fixed amount, while TDMA approach in each frame sets NAV value to $8 \mathrm{~ms}$ or $32 \mathrm{~ms}$ comparing between different vendors. Setting NAV to such values typically suggests two things, it's either HCCA approach without CP period or it's a vulnerability which is used by wireless vendors (Dasari 2017). Since the proposed algorithm does not really change the IEEE 802.11 PHY characteristics, it is possible to implement the algorithm on off-the-shelf IEEE $802.11 \mathrm{n} /$ ac equipment. The wireless medium access mechanism was implemented by re-using some already existing functions on WiFi driver. The activity of the stations are handled based on the throughput and packet per second thresholds. The stations were split into two groups - active and idle. The active stations are considered the ones, which generates more throughput than predefined throughput threshold. The idle stations are kept in Contention Window $(\mathrm{CW})$ period, where they equally compete for wireless medium. If there is any change in the station activity. The decision is made based on the following expression:

$$
P_{i}=\frac{\left(N_{i} \cdot A_{i}\right)}{T_{i}},
$$

where $N_{i}$ - the number of packets in queue, $P_{i}$ - the throughput going through station, $A_{i}$ - the size of the traffic stream from the application, $T_{i}-$ a period during which station is checked for activity.

From Fig. 2.16 it can be noticed that all stations are either polled in RoundRobin method or they are split into two groups. Depending on the total number of active stations a following predefined threshold are shown in Table 2.3, when different number of active stations is registered on AP. 


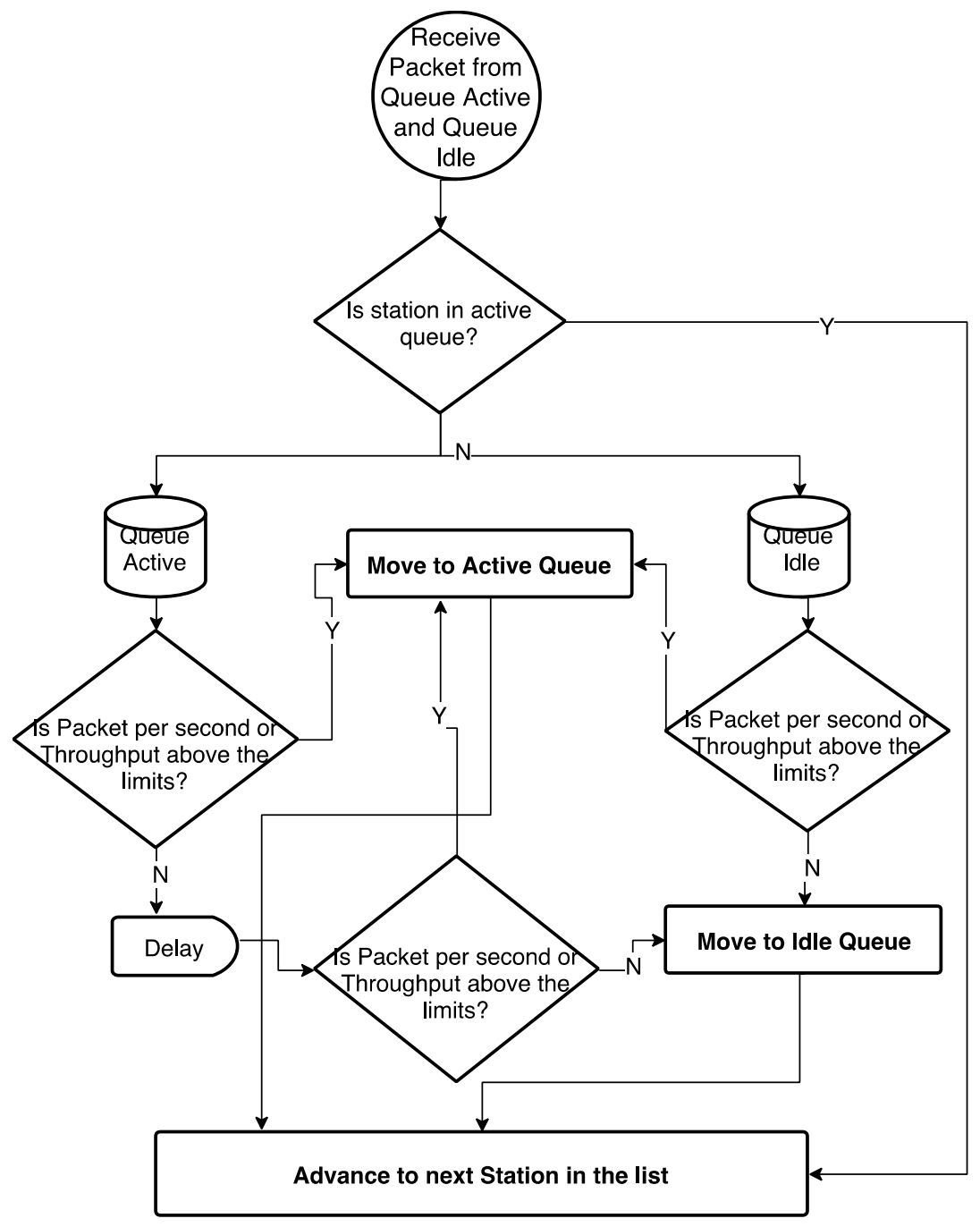

Fig. 2.16. Multi-Poll processing flowchart

Table 2.3. Multi-Poll thresholds for active idle station

\begin{tabular}{|c|c|c|c|}
\hline Number of active stations & Throughput,Mbps & PPS & Delay, s \\
\hline 5 & 1 & 250 & 10 \\
\hline 10 & 0.8 & 200 & 10 \\
\hline 20 & 0.6 & 100 & 20 \\
\hline 60 & 0.4 & 50 & 30 \\
\hline
\end{tabular}


The thresholds have been defined based on the multiple measurements under different conditions and are set as defaults for Multi-Poll protocol, however can be adjusted based on the environmental conditions.

\subsubsection{Rate Control Algorithms in Multi-Poll}

Rate Control Algorithm is mandatory component of Multi-poll, while designing appropriate operation of wireless system. The novel idle and active steering in Multi-Poll protocol is based upon assumption that rate control can handle small amount of collisions until more active stations are steered into the polling list. This is different approach compared to HCCA or PCF where stations are requesting to be polled or are polled constantly even if there is no data. Such polling methods results in management frames that takes additional airtime without sending any useful data.

Over decade there have been many adaptions of rate control algorithms. Based on the input, that is required to make a decision to change the MCS, rate control algorithm can either be based on Packet Error Rate detection, RSSI level detection or a combination of RSSI and PER.

SNR estimation is implemented by re-using RTS/CTS mechanism (Holland et al. 2001). Access Point firstly sends an RTS frame to Station. RTS frame includes the last successful transmission rate and packet size. When station receives RTS frames, it extracts SNR from physical layer and aligns it to all available data rates. Upon selecting the best data rate for current channel conditions, station embeds that suggested rate information into CTS frame and sends it back to Access Point. CTS frame is received by all the neighbor station devices, so they would know how long the transmission is going to last. This is done by extracting data from CTS frame data rate information field and frame size. Tentative channel reservation time is started for all the stations, which received CTS frame. The final decision what transmission rate should be used is still made on Access Point side, since CTS only includes suggestion of possible data rate. While this seems to be efficient for estimating channel conditions and selecting appropriate data rate, it includes a lot of overhead by using RTS/CTS mechanism, which takes additional airtime and thus decreases overall performance. Additionally, by reserving transmission airtime, individual stations might drag total capacity of Access Point down. This especially noticeable wireless network contains stations with varying data rates. Similar performances degradations have been reported by Heusse (2003).

SINR based algorithms have primarily sampling problem, due to insufficient sampling on signal level. Additionally, each station device might have different antenna and thus would result in case that selected rate is not optimal or not even usable at all. Multi-path causes even more harm to SNR estimation mechanism, 
since received signal level might have difference up to $14 \mathrm{~dB}$ at different times (Brodsky, Morris 2009).

Next an attempt to correlate Received Signal Strength and data rate was introduced by Pavon, Choi (2003). Algorithm tries to relate these two parameters together by monitoring all transmitted frames. The RSSI is stored on each device which receives data frames and is used to select appropriate data rate. RSSI is updated from last received packet. A constantly updated transmission rate, which provides fast data rate adaption to changing channel conditions is required. However, when high packet per second is transmitted this would heavily hit the performance due to constant packet processing. Similar approach was taken by $\mathrm{Wu}$ et al. (2007), instead of sensing all frames, authors propose to sense only beacon frames. However, this approach is inappropriate for two reasons. Beacon usually is transmitted at lowest modulation, therefore it's expect to have the highest possible RSSI value that is extracted from beacon frame, while data packets are typically sent at higher modulation, which have lower RSSI.

In summary, RSSI based approaches are typically avoided, since it's hard to estimate proper correlation between transmission rate and SNR or RSSI values. However, Hauer et al. (2007) RSSI might be used as a interference detection parameter. In article it's stated that while packet collisions happen, temporal increase of RSSI can be seen. From such increase authors can identify the cause of packet corruption as well as estimate bit error positions. Furthermore, another authors claims that RSSI is good estimator if calculated as an average of all received packets (Srinivasan, Levis 2006).

Frame loss based algorithms typically do not show such problems as RSSI based algorithms. Atheros uses frame loss based algorithm (Wong et al. 2004). Important moment is that frame based algorithms typically do not evaluate if frame loss occurred due to collision or due to channel fading. Therefore, if scenario has multiple collisions between different stations, rate control algorithm would decrease data rate. However, in such cases instead of decreasing collision probability it's being increased, therefore a chain reaction occurs and all the transmitting stations starts to send at lowest MCS, which results in closed loop between collisions and rate control algorithm.

Adaptive Multi Rate control algorithm (AMRR) uses a BEB to change the values of the rate/count pairs. In short, AMRR exposes multi rate retry mechanism and sets how long a packet has to be retried at certain data rate before decrease to another one. Since multi rate retry mechanism, can firstly retry the same packet four times at current rate and only then decrease. AMRR selected a more sensitive approach by decreasing rate/count pair value to 1 , which means, that upon packet loss, packet is retried only one and immediately tried on the lower rates.

LDRA rate control algorithm (Biaz, Wu 2008) attempts to retransmit failed packets at lowest possible rate, if they are successful it's considered as channel 
fading effect. Otherwise rate remains unchanged. Evolution of LDRA is ERA (Wu, Biaz 2008) based rate control algorithm, which as well considers channel fading if short frames fail to reach its destination. The assumption by authors is that short frames have higher probability to reach end destination because they require short transmission time and thus probability of collision is lower. ERA starts by using intermediate frame, or the middle between lowest and highest possible data rates. Fragmentation is used upon unsuccessful frame transmission to split the actual frame into smaller and bigger parts, thus assumption is if it was collision smaller frame will be able to reach receiver. In order to probe higher rates, ERA uses same approach as AARF method, if $N$ packets reach successfully receiver ERA tries to increase data rate to the next, otherwise threshold of required packets is increased and rate is set to the prior selected data rate. Despite there being many rate control algorithm suggestions, most of them are still aren't used due to complexity and only standard ones are being used by Qualcomm and other vendors.

Qualcomm Atheros provides interesting approach to different user applications, including the full stack of wireless driver and some advanced algorithms. Rate control algorithm, which is implemented by Atheros is an open-loop auto rate mechanism. Open-loop means, that rate control does not add any modification to standardized control frames, thus compatibility is still intact. The rate control module can be called hybrid since it evaluates both RSSI and PER. Similar approaches have been made by other authors in (Haratcherev, Langendoen 2004), almost exact same functionality is done by Atheros.

Atheros rate control module uses a table which includes RSSI, PER and UDP rates for each support data rate. These values are updated based on actual transmission information. According to Atheros patent (Wong et al. 2004), rate control firstly extracts RSSI value from ACKs frame and calculates either average value of several packets or determines a media value of RSSIs from three ACK packets. After that it navigates through a table and locates rates which are equal or higher than required RSSI value. As stated initial RSSI threshold are theoretical minimum, later in operation these values are updated according to gathered statistics. Achievable throughput figures on selected rates are calculated for UDP, which doesn't have ACKs as TCP has, therefore it's easier for computation.

Adaptive rate control can further determine if a packet size is greater than some defined value, if the packet is greater than some defined value, RSSI which is extracted from transmitter's receive descriptor can be decreased by $1 \mathrm{~dB}$ before searching for usable data rate. This is done with assumption that longer packet are more prone to error, therefore they decrease extracted RSSI by $1 \mathrm{~dB}$. If the packet is relatively short, then RSSI value is not changed.

Additionally, rate control has a probe rate mechanism, which constantly checks if rate module can use higher data rate by probing higher rate with single 
packet. If probe rate is successful, rate is increased. As stated in Wong et al. (2004) probe rate is successful if one or two frames are transmitted without errors.

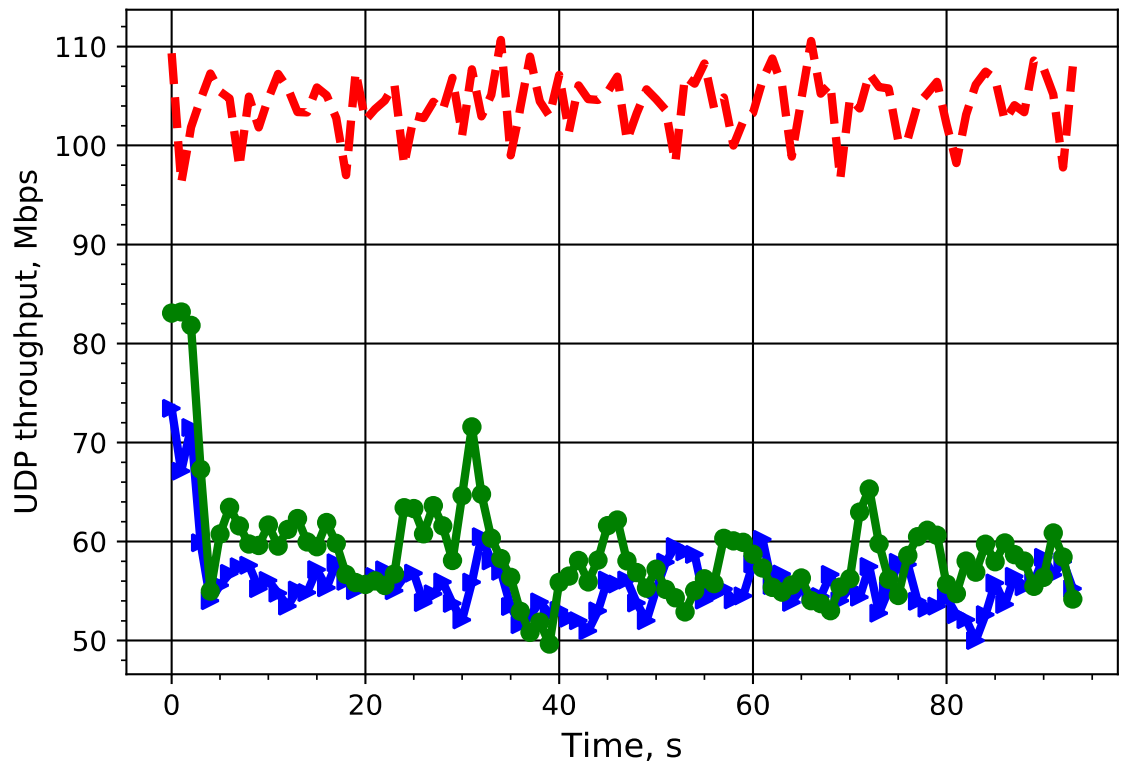

$\rightarrow$ PER Rate Algorithm
- Hybrid RSSI Rate Algorithm

Mixed Rate Algorithm

Fig. 2.17. Universal Datagram Protocol throughput through 74 connected stations in Point-to-Multipoint scenario, when packet error rate and Received signal strength indication based rate control algorithms are used

Furthermore, adaptive rate technique can update its rate table depending if there are excessive retries for current transmission rate or not. Excessive retries is considered as a case where none of retransmissions are successful. Default rate module has 4 hardware retries and additional one software retry, so fives retransmissions with error are considered excessive retries. In case there are excessing retransmissions, PER and RSSI values can be modified in real-time and thus PER is increased by 0.35 for that transmission rate. Excessive retries causes significant throughput decrease and overall system's capacity decrease. Such transmission rate which fails with excessive retries is considered poisoned and is not being used in further calculations.

In order to evaluate how the different rate control algorithms are acts, a point to multipoint network topology has been created. The tests are started in outdoor 
environment, where multiple third party vendors are present. Unless stated otherwise, all tests were performed with standard $802.11 \mathrm{n}$ mode. The testbed consists of Qualcomm AR9342 chipset based devices with CPU processing power $533 \mathrm{MHz}$. All, 78 devices and Access Point are based on IEEE 802.11n 2×2 MiMo with 64 QAM modulation capability. Stations, which are connected to Access Point, have respectively $15 \mathrm{dBi}$ and $20 \mathrm{dBi}$ antennas, Access Point has 90 degree $18 \mathrm{dBi}$ sector antenna. The distance between clients and AP is $200 \mathrm{~m}$ and $5 \mathrm{~km}$ respectively. The channel width is $40 \mathrm{MHz}$ and frequency is set to $5430 \mathrm{MHz}$.

In the test, performance of different rate control algorithms was measured when a high density of stations are operating in point to multipoint scenario. In this case, all the stations were transmitting and receiving data at the same time. Traffic generator Nepim was used for bidirectional traffic generation. In total, 78 stations were transmitting and receiving UDP traffic at the same time, individually it was assigned to generate $3 \mathrm{Mbps}$ bidirectional per station device. Some of the stations are hidden wireless nodes, thus creating multiple collisions due to hidden wireless node problem. From Fig. 2.17, it can be seen that PER based algorithm is less efficient when dealing with high number of collisions. This is mainly due to inability of PER to identify the cause of actual collision. It can be stated that hybrid RSSI rate control provides up to $50 \%$ better efficiency in high density PtMP scenario and provides better robustness against collisions between individual stations.

\subsubsection{Game Theory based Power Allocation in Multi-Poll}

In order to build an efficient multi-band system an effective power allocation mechanism has to be build. Since the environment and operation factors in wireless systems are very dynamic, typical power control approaches when a static value is being set can't accomplish the needed transmit power control. Therefore, it is mandatory to build a power control allocation mechanism which would be able to adapt to the environmental changes and would be both simple and effective in all operational scenarios.

The essence of Game Theory (GT) modelling is that each device has to be considered a selfish player, where player is a wireless station connected to the AP. Player's interactions are strategic in a sense that given player's payoff depends on the other players' actions. Any game model can be classified into different categories based on the number of players, the sum of gains, and the strategies or data types (Felegyhazi, Hubaux 2006; Benmammar, Krief 2014). Since wireless resources especially in Wi-Fi are shared between multiple instances of devices noncooperative game is the one, which can handle such complex scenarios. The cooperative game would require additional signaling protocol implementation for each player due to the fact that advanced planning for this type of game is required. 
Therefore, it is easier to adapt non-cooperative game models in highly dynamic wireless environment, so it would be possible to avoid usage of interconnections between wireless devices. The non-cooperative game can be classified into two methods: strategic game or extensive form game (Felegyhazi, Hubaux 2006). The difference between two methods is related to amount of information, which can be passed to player. In the strategic game, all the players and their strategies are known while in the extensive type most of the attention is placed on timing and amount of information that is available to each player. Players are considered rational and follow their strategy to maximize their payoff function. Since Wi-Fi operates in shared wireless medium only Multiple Access Game is considered (Felegyhazi, Hubaux 2006; Benmammar, Krief 2014), where each player tries to send some data frames. The packet transmission is successful if player does not transmit any data while other player has occupied time slot for a transmission. If there are no collisions then player, which waits for opportunity receives a reward of 1 from successful packet transmission. This type of Game can be considered as nonzero-sum game where each player's gain or loss is balanced by the other player's loss or gain of utility (Felegyhazi, Hubaux 2006; Benmammar, Krief 2014). IEEE 802.11 wireless devices usually do not coordinate each other actions, the only way to notice change of other devices is from the environment monitoring statistics. After the game is defined, the game has to be solved so it would be possible to know each player's strategies. Simple games can be solved by iterated dominance (Felegyhazi, Hubaux 2006), however not all games can be solved by this technique.

A number of GT algorithms and protocols had been proposed for optimizing channel assignment and power allocation (Wang et al. 2010; Kazemi et al. 2010). Standard analytical models no longer cope with the increasing complexity and dynamics wireless systems environment. Whereas GT provides the framework in which the paradigm shift to more flexible and efficient resource sharing may eventually materialize. In the proposed game the power allocation is determined as the outcome, hence representing a distributed, interference-aware, power allocation game. In a wireless network of $N$ independent transmit-receive pairs $\left(\mathrm{Tx}_{\mathrm{i}}-\mathrm{Rx}_{\mathrm{i}}\right)$, each pair would be considered a player in the game. The objective is to find stable points of power allocation for each player such that the players' global utility is maximized while the cumulated power levels are kept to a minimum.

When regarding the composition of players, it is also important to consider master-slave relationship aspect. In the context of Wi-Fi operation, the game models a set of primary master devices - such as APs, each having one or more stations.

The APs and it's stations are competing between each-other for a share of wireless medium. The stations are implicitly appearing as a secondary tier and do not need to be part of the game strategy. In the case an AP has more than one 
station, it could use its own scheduling to partition available wireless medium between its served stations, which is in line with proposed wireless medium access scheme. This is especially important when handling multiple APs deployments at infrastructural network topology, which requires frequency re-use (Yang et al. 2015). The frequency re-use requires additional protocol (Chaudet, Yaddad 2013) changes as well as efficient power management between APs and stations, so they would minimize the effects of self-interference when operating on the same tower by re-using small chunk of spectrum allocated to specific WISP.

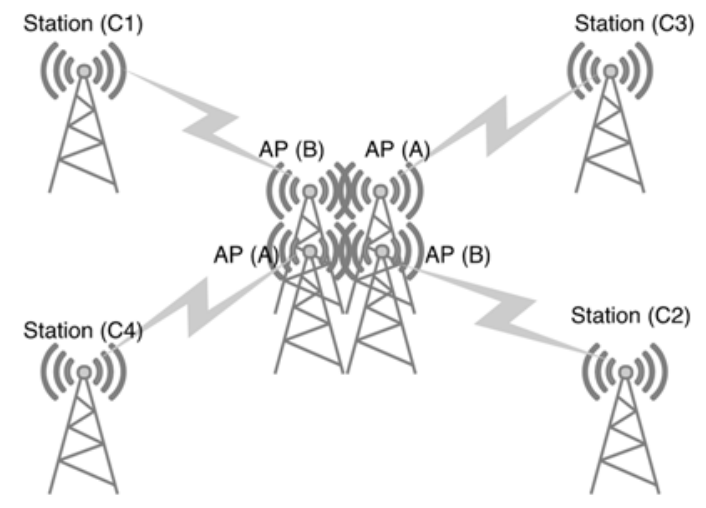

Fig. 2.18. Access points collaboration when frequency re-use is applied

The game only needs to simulate a sub-set of finite size from a total population of APs that might be located in a given area. The coexistence community would be made of APs that share a common part of spectrum, i.e. a given channel. It may also change with time, if the channel is changing as a result of using Dynamic Frequency Selection mechanism or otherwise. In Fig. 2.18 a typical frequency re-use deployment is shown, where different APs are sharing same frequency. In example, A APs are using $5500 \mathrm{MHz}$ frequency and B APs are reusing $5520 \mathrm{MHz}$ frequency with possible channel width of $20 \mathrm{MHz}$. As it is noticeable the important part here is the Tx, Rx synchronization between A group APs and B group APs this is achieved by implemented TDMA based protocol which handles the synchronous transmission, however in such deployment the transmit power from stations: $\mathrm{C} 1, \mathrm{C} 2, \mathrm{C} 3, \mathrm{C} 4$ plays an important by minimizing the effects of far end and near end interference.

It is known that antennas can still hear some part of the noise coming from station $\mathrm{C} 1$ to $\mathrm{AP}$, which transmits to station $\mathrm{C} 2$; therefore, it's important to use power control allocation between clients of far and near end. This is important 
part of proposed game theory model. The proposed power coordination game was built and tested with the following key objectives in mind:

1. To ensure self-governed access to the shared (ISM) bands with the objective of improving the global spectral-spatial efficiency of the band, e.g., measured as the total aggregate traffic of all links in given locality/area.

2. To ensure that newly deployed devices built on the GT operating paradigm do not create excessive interference and disruptions to the legacy CSMA/CA-based devices. In fact, the latter devices might still well participate passively in the channel access game to certain extent, being perceived as players with fixed strategies. There will though remain a risk that the legacy devices might become victims of "exposed node" phenomenon and be effectively prohibited from attempting transmission (Medeisis et. al 2014). At randomized time instances, each AP evaluates its Signal to Interference plus Noise Ratio (SINR) and if it has changed since previous check, calculates the new transmit power, which is inversely proportional to actual SINR (Medeisis et. al 2014).

The APs will be constantly in the state of balanced transmit power versus background noise, which should ensure the instantaneous transmission of message using random channel access. In fact, this means that Wi-Fi devices could be also transmitting incessantly, which was actually how the process was simulated when establishing total achievable aggregated data throughputs.

Note that under the proposed power coordination game, the active collaboration between AP is strictly speaking not necessary as a departing point and the simulations reported herein were done with agents acting without communicating with each other. The main particularities of ISM/RLAN operation come from the fact that the band is unlicensed, incurring uncoordinated deployment, high-density, open access, opportunistic behavior, and, at some point, spectrum congestion. It is a dynamic environment, difficult to analyze and for which it is difficult to provide sound resource management schemes. Standard analytical models no longer cope with the increasing complexity and dynamics of nowadays communications systems. GT provides the framework in which the paradigm shift to more flexible and efficient resource sharing may eventually materialize.

Within this framework, the key problem is to design distributed resource allocation rules that lead to a Nash equilibrium that is efficient and possibly fair (Wang et. al 2010; Etkin et. al 2010; Belmega et al. 2010). These rules would be self-enforcing and therefore not requiring external intervention to verify compliance (Etkin et al. 2010).

The resource allocation is determined as the outcome of a game; a distributed, interference-aware, power allocation game. Given a wireless network of $N$ transmit-receive pairs $\left(\mathrm{Tx}_{i}-\mathrm{Rx}_{i}\right)$ APs, where a "pair" is referred to as a "player", let's subject it to a GT analysis where the objective is to find stable points of power 
allocation for each player such that the players' global utility is maximum while the cumulated power levels are kept to a minimum. More formally, given a set of $N$ players (or $N$ Tx-Rx pairs) $N=\{1,2, \ldots N\}$ and their corresponding power allocation profile $P=\left\{p_{1}, p_{2}, \ldots p_{n}\right\}$, the utility function of each player (Tx-Rx pair) is given by:

$$
u_{i}=\log \left(1+\frac{h_{i i} p_{i}}{n_{0}+\frac{1}{B} \sum_{j \neq i} h_{j i} p_{j}}\right),
$$

where $p_{i}, p_{j}$ are the transmit powers of players $i$ and $j, h_{i i}$ is the direct gain, $h_{j i}$ is the channel gain between transmitter $j$ and receiver $i, B$ is the total channel bandwidth and $n_{0}$ is the noise power.

User $i_{t h}$ received SINR at the receiver side is:

$$
r_{i}=\frac{h_{i i} p_{i}}{\frac{1}{B} \sum_{j \neq i} h_{j i} p_{j}+n_{0}} .
$$

The objective is to maximize the global utility function, $\max \sum_{i} u_{i}$ while minimizing the globally allocated power $\min \sum_{i} p_{i}$ where $p_{i} \in\left[0, P_{i}^{\max }\right]$.

The convergence and stability condition may be given as (Fang et al. 2010):

$$
\left|\frac{h_{j i}}{h_{i i}}\right|<\frac{1}{N}, i=1, \ldots, N .
$$

This condition is a decisive factor when choosing the topology on which the power allocation game is implemented. If it is not fulfilled for all players, there will be no strategy profile that will satisfy the players.

In the theoretical case, considering (2.7) below, equilibrium is reached in the game when $p_{i}(t-1)=p_{i}(t)$ for all players at once:

$$
b_{i}\left(p_{-i}\right)=\frac{1}{c_{i}}-\frac{\sum_{j \neq i} h_{j i} p_{j}+n_{0}}{h_{i i}}=\frac{1}{c_{i}}-\frac{I+n_{0}}{h_{i i}},
$$

where $b_{i}\left(p_{-i}\right)$ represents the best response of device $i$ given the current state of the game (the power profile for all other devices is denoted by $p_{-i}, c_{i}$ represents device $i$ 's energy cost, $h_{i j}$ are the channel gains, $p_{j}$ is the transmitted power for all the other devices, and $n_{0}$ is the noise. It should be noted that the choice of the $c_{i}$ 
parameter is critical as it sets the ultimate convergence level in the above equation. This parameter, the energy cost, is the limiting factor precluding the player to raise its power indefinitely. Its values below 1 would mean that the energy cost is little; such as, e.g., may be seen in the case of mains powered APs. On the other hand, the battery driven portable devices represent a good example of high energy costs (above 1), as excessive use of increased transmit power would lead to fast depletion of the battery.

Note that (2.7) will thus represent the best response for any of the players (equivalent to the Nash equilibrium condition). However, in practice, where $\mathrm{p}_{\mathrm{i}}$ can take only discrete values, an experimental, robust stopping criterion is needed in order to determine reaching of the equilibrium. A criterion may be given by comparing the difference between the last $k$ best responses with a threshold power used to compensate for the environment dynamics, $P_{t h}$, and $k$ is determined experimentally:

$$
\left|b_{i-k}\left(p_{-i}\right)-b_{i}\left(p_{-i}\right)\right|<P_{t h} .
$$

Based on the above, the following power control protocol operation consists of these steps:

Step 1: Devices initialize their transmit powers $p_{i}^{0}, p_{-i}^{0}$;

Step 2: If device $i$ updates its power, it will alert the neighbouring devices that a power change has been made. This sharing of current state may be achieved also by sensing;

Step 3: If device $i$ detects a change in neighbouring devices' powers, it updates its power according to

$$
b_{i}\left(p_{-i}\right)=\frac{1}{c_{i}}-\frac{\sum_{j \neq i} h_{j i} p_{j}+n_{0}}{h_{i i}} .
$$

and alerts the neighbours of its own change (or they notice it by sensing).

Step 4: Device $i$ checks if the Nash equilibrium condition is satisfied;

Step 5: If the Nash equilibrium is reached, the game is stopped. The devices maintain NE as this is a situation they have no incentive to unilaterally deviate from - it may be seen as a self-enforcing rule (Etkin et al. 2007).

Simulation of the power allocation game was conducted using the above described algorithm modelled by developing a bespoke software code (Python programming simulator). Parameters used for simulation were as follows: $n_{0}=10^{-12}$, $c_{i}=1$ for all users, $h_{i j}$ were calculated using ITU indoor propagation model P.1238 (ITU-R Recommendation P.1238 2001), number of users was varied between 2 and 14 and the main result was expressed as a function of total system capacity (i.e. sum of capacities of all individual links) on number of users in the system. 
Transmitters, each corresponding to one user, were distributed randomly with uniform density in a $100 \times 100 \mathrm{~m}$ square and each was assigned a corresponding receiver, placed within a $40 \times 40 \mathrm{~m}$ square around that transmitter.
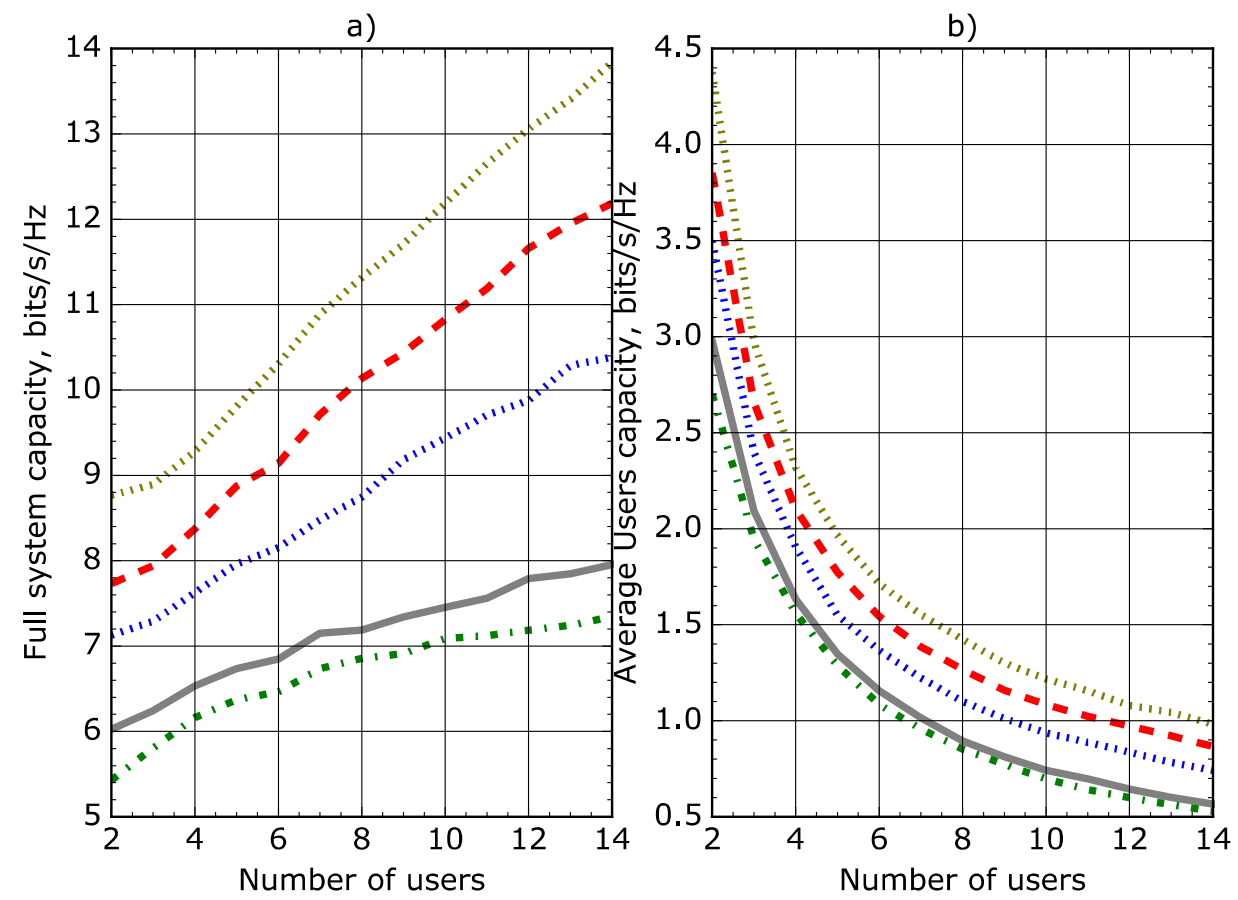

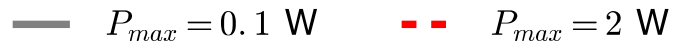

$$
\begin{aligned}
& \because P_{\max }=0.2 \mathrm{~W} \quad \cdots \quad P_{\max }=4 \mathrm{~W} \\
& \text { ㄴ, } P_{\max }=1 \mathrm{~W}
\end{aligned}
$$

Fig. 2.19. Aggregate channel-area capacity for Wi-Fi devices employing interference-aware power coordination game $(100 \times 100 \mathrm{~m}$ area) a) Full Systems Capacity b) Average User Capacity

Each simulation was repeated 5000 times (using different randomly generated user positions) and average total capacity was derived in Fig. 2.19. The simulation results are especially striking noting the rather small scale of the simulated scenario, where higher power would be normally not considered necessary thanks to favorable link budget over such short distances. This illustrates that removing the artificial EIRP limit would allow Wi-Fi devices to tap into additional power 
margin when needing to improve SINR and subsequently link quality. At the same time these results prove that given the clear and simple power game rules, the system would converge to some equilibrium state and no excessive over-exploitation of common band would occur. It's also worth to note, that a working task group of IEEE 802.11ax defined a new set of rules for wireless medium access. Which includes the adaptive Clear Channel Assessment (CCA) mechanism in Fig. 2.20.

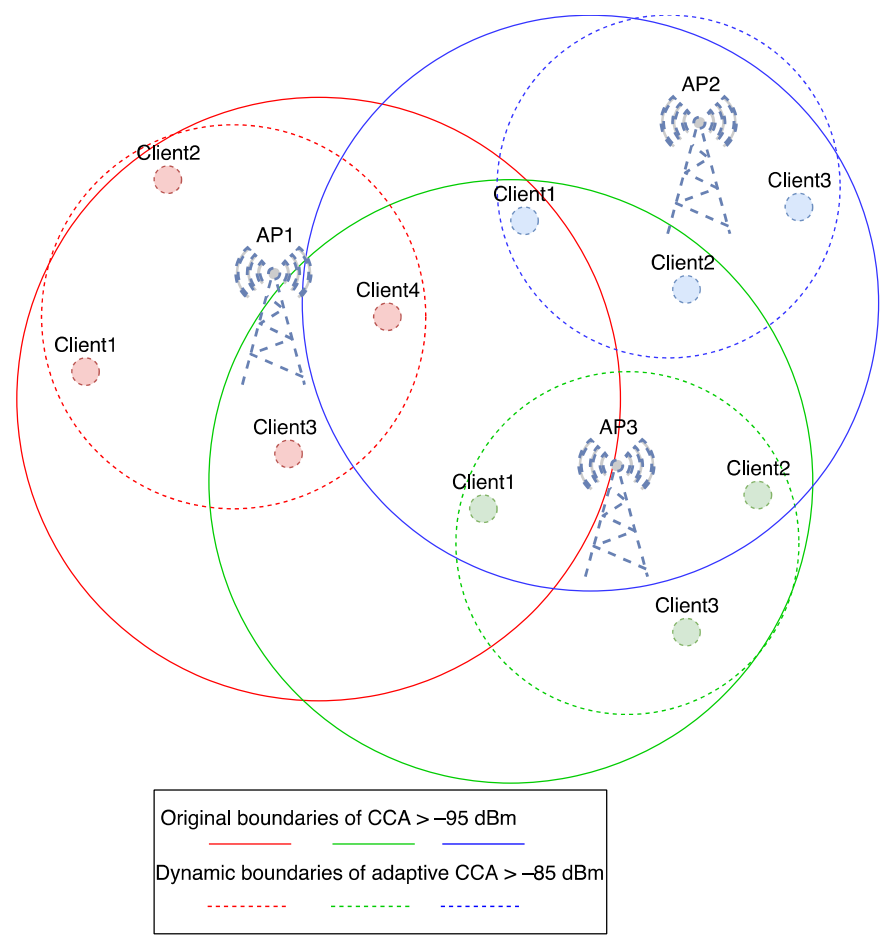

Fig. 2.20. Dynamic Clear channel Assessment boundaries allocation

CCA in current IEEE 802.11 standard is fixed and required by all the operating devices, however it's been quite some time noticed from State-of-the-art devices that they highly modify the operation of IEEE 802.11 standard in outdoor based products. One of such modification is a dynamic adaptivity of CCA (Khorov et al. 2016). The modified IEEE 802.11 adapts the CCA receiver's sensitivity level based on the worst client's signal level, thus all the noise which is below the worst client's signal level is eliminated. For instance if operating stations hear any frame from third party wireless devices they perform now a back off even if the signal was below for say $-85 \mathrm{dBm}$, thus all the operating wireless devices can set $\mathrm{CCA}>-85 \mathrm{dBm}$ and decrease the potential noise from nearby, this would improve both the number of retries and roaming possibilities (Haghani et al. 2010; Shen 
et al. 2009). The dynamic boundaries would elevate the usage of higher transmit power and is in line of solving both, the possibility to decrease the sensitivity level based on the last client device and increase the propagation at the same time, higher transmit power would allow to decrease possibility of hidden node when dynamic CCA is being used. After modifying the game simulator in order to measure average user transmit power and total system capacity, an average transmit power was verified for each user. A 1000 possible snapshots for each scenario was generated and from Fig. 2.21. It can be seen that Average Power per User is directly related to number of players in the game.

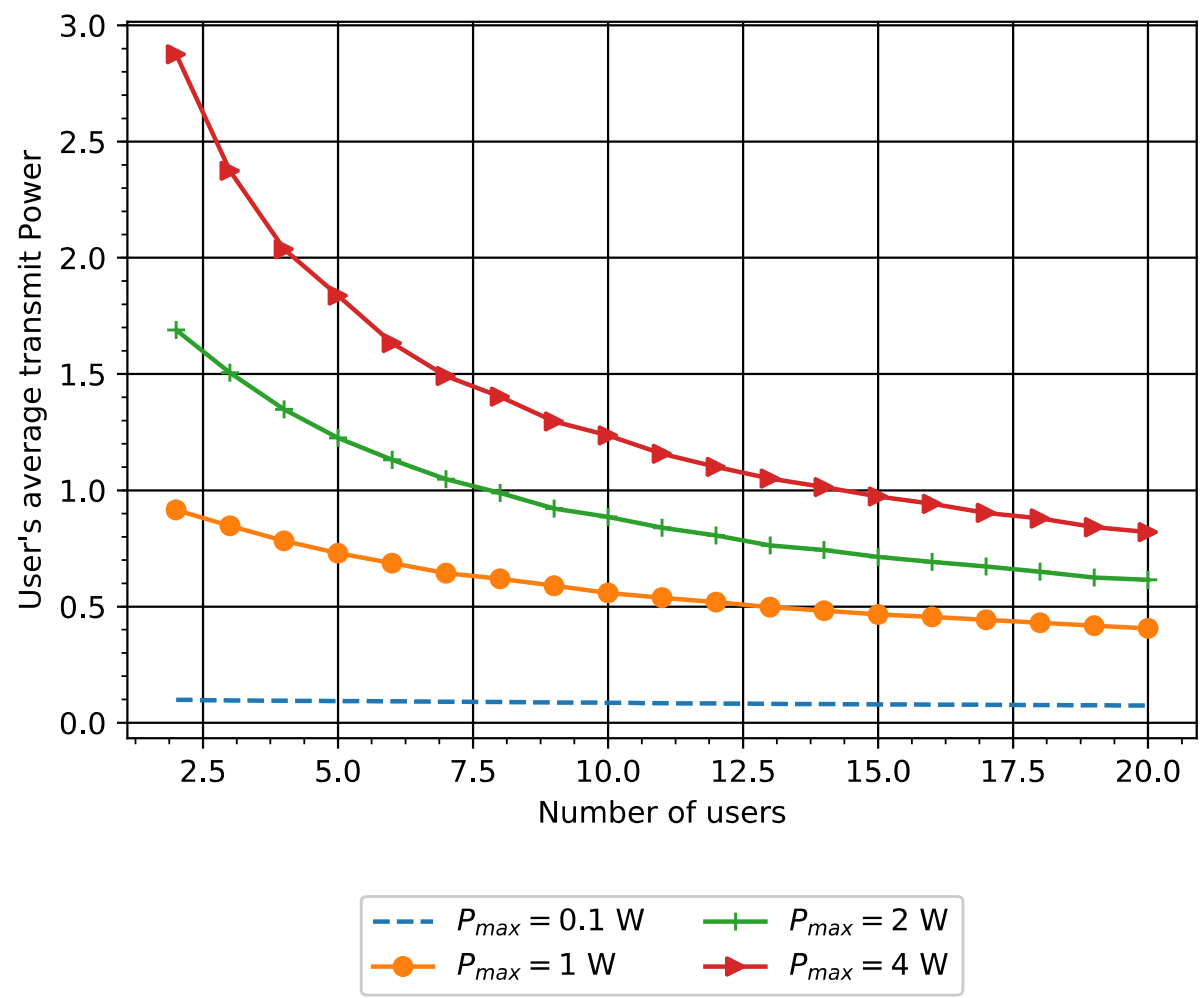

Fig. 2.21. Average User's transmit Power as a function of number of users $(40 \times 40 \mathrm{~m}$ area $)$

When number of players in the game (Tx-Rx pairs) increases, the average power per user decreases and results in lower interference for all the existing players. The power levels never reached the $P_{\max }$ even with low number of players in the area. The trade-off between utility and power function can be controlled with 
power cost factor. As can be seen from Fig. 2.22, the selected different power cost values for the same maximal transmit power allows user to select different transmit power. By adjusting the cost factor, the Average User's transmitter Power can be limited together with ability to control their utility function. By decreasing the power cost, user is allowed to set higher transmit power, which results in higher utility. Opposite to low cost for power by setting higher cost for power utility function is decreased for users.

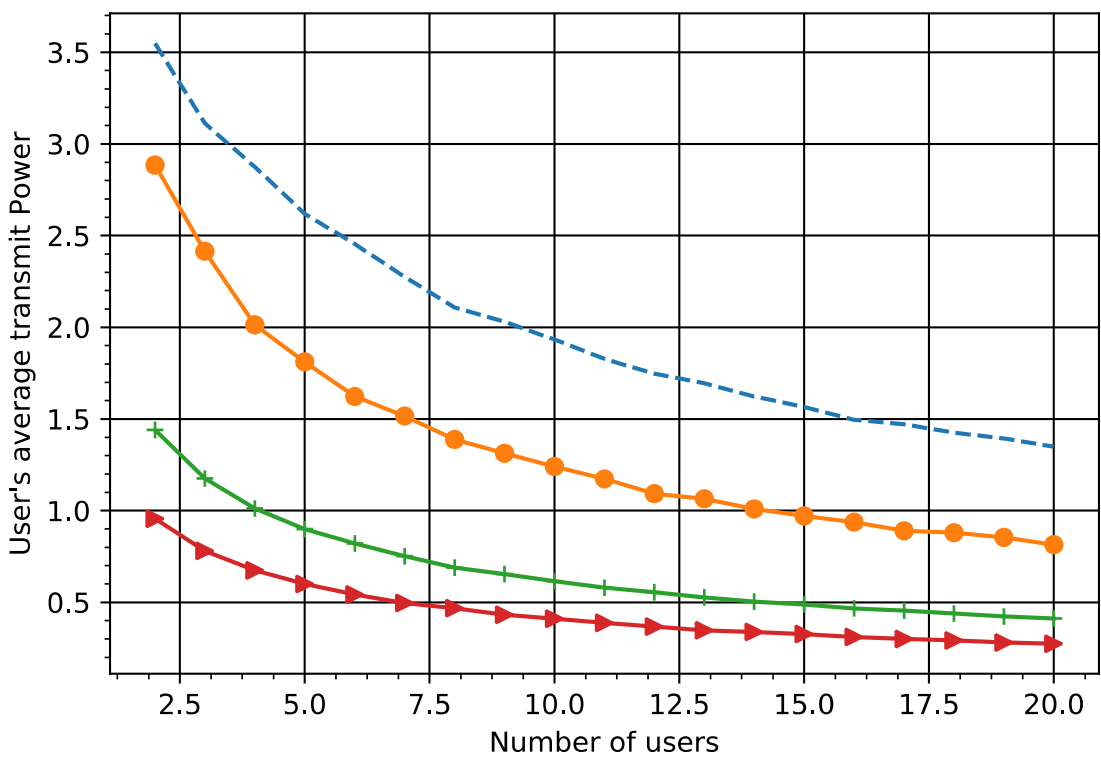

$$
\begin{aligned}
& --P_{\max }=4 \mathrm{~W} \text { with cost } 0.1 \quad \longrightarrow P_{\max }=4 \mathrm{~W} \text { with cost } 0.5 \\
& --P_{\max }=4 \mathrm{~W} \text { with cost } 0.25 \longrightarrow P_{\max }=4 \mathrm{~W} \text { with cost } 0.75
\end{aligned}
$$

Fig. 2.22. Average User's transmit Power as a Function of Power Cost ( $40 \times 40 \mathrm{~m}$ area)

Furthermore, having the devices with highly directional antennas might allow addressing the fairness more "intelligently" because of decreased noise level. For this comparative simulation a new metric was used, by expressing the results in terms of "user satisfaction" of Wi-Fi devices, i.e. the percentage of users who achieved their throughput requirements.

Finally, a reference simulation was performed in Fig. 2.23, where all devices would be either power-restricted users as now, or GT-enabled users. For this comparative simulation a new metric was used, by expressing the results in terms of "user satisfaction" of Wi-Fi devices, i.e. the percentage of users who achieved 
their throughput requirements. The results obtained for homogeneous clusters that consist of solely one type of devices are shown. The simulated results shown illustrate the well understood (and already observed in real life) tendency that the growing number of closely co-located users reduces the effective throughput available to individual link. However, the GT-enabled users on average show some $20 \%$ higher satisfaction rates. The next simulation in Fig. 2.24 considered mixed scenario with varying proportion of GT-enabled versus legacy Wi-Fi devices. The results show that the reducing proportion of incumbent $100 \mathrm{~mW}$ devices would mean proportional decline of link availability for their users, up to $20 \%$ for "last man standing", due to proliferation of more power agile GT-enabled devices. Still this would be offset by the growth of total link availability for the larger community of devices.

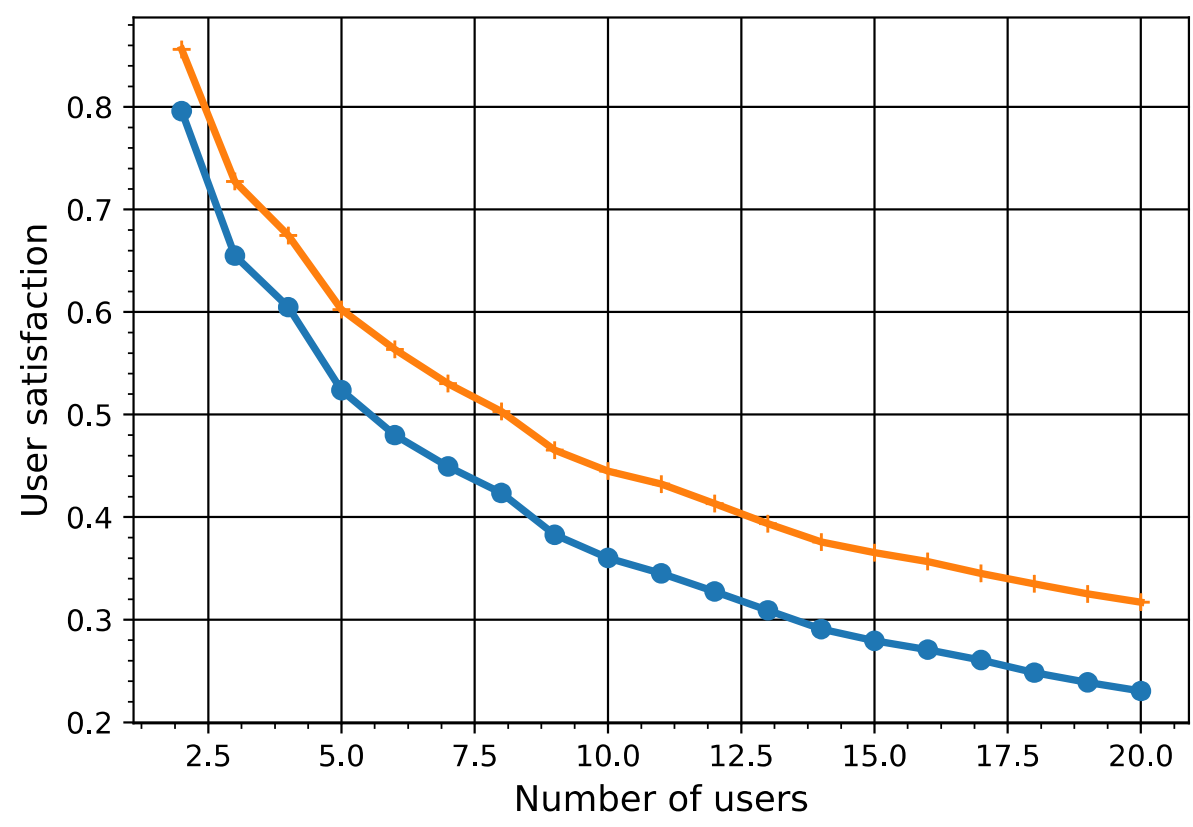

Satisfation of max power users

Satisfaction of GT users

Fig. 2.23. Achieved individual data throughput objectives: homogeneous device cluster in $(100 \times 100 \mathrm{~m}$ area $)$ 


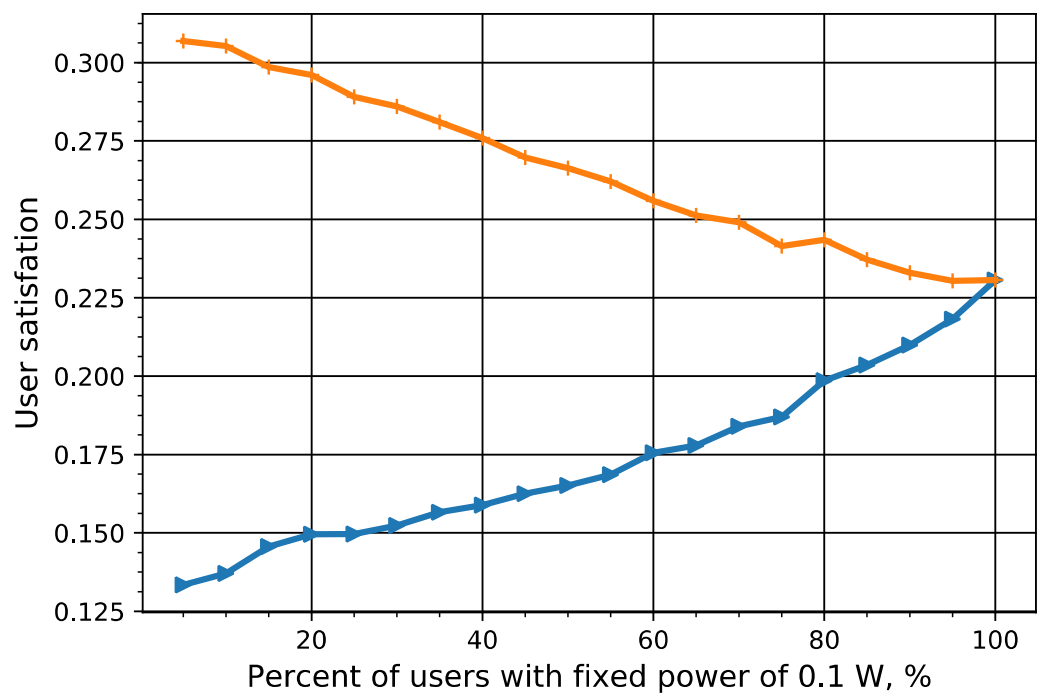

Average satisfaction of users using fixed $0.1 \mathrm{~W}$

Average satisfaction of all users

Fig. 2.24. Achieved individual data throughput objectives: mixed device cluster in $(100 \times 100 \mathrm{~m}$ area $)$

Most importantly, the suffering of incumbent devices was shown to be minor; far from pessimistic scenario of total shutdown due to over-dominance of much more powerful GT-enabled devices.

\subsection{Conclusions of Chapter 2}

1. Key components of Multi-band system have been identified. The main components include the wireless medium sharing mechanism, rate control modules as well as power allocation algorithms. It has been pointed that up-to-date existing IEEE 802.11 standard can't cope with upcoming demand for higher efficiency in high density outdoor deployments. Therefore, a new wireless medium access protocol is required, which would increase system's capacity.

2. It has been identified that new wireless medium can use a control frames, that use a smart rate adaption algorithm to cope with loss or packet retry. Thus, the control frames while being sent at the highest modulation provides lower airtime usage up to 10 times compared to PCF CFP polling 
frames with assumption that there is more than 1500 polling frames. This allows to conclude saved airtime can be used for real data transmission between APs and clients. This can be compared both to existing EDCA implementations as well as TDMA approach, which also uses low modulation coding schemes for management frames to poll clients. Unlike the traditional EDCA approach, new Multi-Poll proposes to use combination of active polling and EDCA usage. This is mainly to cover the bursty traffic patterns, which are common between usual wireless network users. Additionally, by using combination of EDCA and active polling there's possibility to gain higher TCP performance when number of active users is low.

3. It has been experimentally proven that current State-of-the-art chipsets using IEEE $802.11 \mathrm{n}$ by using advanced rate control modules are able to handle up to $10 \%$ continuous of hidden wireless node traffic, which provides a further establishment for Multi-Poll wireless medium access scheme. This provides a base for active and inactive client steering capabilities based on their current throughput patterns.

4. Experimentally a new set of rate control module, which is called hybrid rate control module was evaluated. A combination of PER based and RSSI based rate control module, can provide a background to handle low impact of collisions, which would prevent the sudden modulation drop. This is especially needed when rate is dropped just based on the collision event in wireless medium and not the actual channel condition changes. The test has been performed with 78 client set, which operated at different distance ranging from 200 meters up to $5 \mathrm{~km}$. The hybrid rate control algorithm shows up to 50\% higher efficiency, when PER and RSSI are being used based on the device type, in such case AP uses PER based rate control algorithm and station devices due to their fixed position uses RSSI based algorithm.

5. Since current IEEE 802.11 is limited by the actual EIRP which can be used a lighter transmit power rules might increase the efficiency and proper power allocation mechanism is crucial in the efficiency wireless network deployment. A Game Theory approach for power control in distributed wireless networks was researched which is a part of the multiband wireless system. It has been identified through Game Theory modelling that up to $20 \%$ higher satisfaction rates are gained by the users who uses Game Theory based power allocation method. The simulated results show tendency that the growing number of closely co-located users reduces the effective throughput available to individual link. However, the GT-enabled users on average show some $20 \%$ higher satisfaction rates. 
6. Differently than fixed transmit power users, GT based users provide far better results and increases the satisfaction up to 0.25 depending on the cost coefficient. When amount of users with $100 \mathrm{~mW}$ limitation reaches $100 \%$, overall user satisfaction increases. In different set of environment GT based devices can provide higher user satisfaction as well as have very similar user satisfaction in dense environment. 



\section{Experimental Investigation of Multi-band Wireless System}

This Chapter examines the efficiency on Multi-Poll based protocol in dense wireless networks and potential usage of Game Theory approach for efficient Power Allocation. The measurements performed in this section examines the Multi-Poll protocol practical usage scenario and are compared to the currently used wireless medium access schemes, that are incorporated into off-the-shelf devices.

The practical measurements are performed in order to confirm the actual performance of the proposed wireless medium scheme in previous chapter and confirm its operation in common scenarios, which includes hidden node topology, high density wireless network topology when there are multiple active and idle clients. The measurements were performed in outdoor based location, in Vilnius city, where a complete set of devices were installed at different distances. The setup consisted of 83 clients total and 2 APs.

The material presented in this chapter was published in (Cuzanauskas, Anskaitis 2015a; Cuzanauskas, Anskaitis 2016a; Cuzanauskas, Haddad 2017a) and presented in one international conference: (Riga 2015). 


\subsection{Aim of Experimental Investigations}

This section evaluates the practical use cases of multi-band system and its components. The experimental investigation continues the performance measurement of wireless medium access protocol as well as multi-band application in practical use case. The section evaluates these main parts:

1. Multi-Poll wireless medium access scheme operation in dense wireless networks. The Multi-Poll WM scheme is based on the assumption to handle high number of operational stations in dynamic wireless environment as well as provide possibility to handle triple play services, which would include standard data as well as voice and video service possibility. The aim is to assess the performance of Multi-poll WM access scheme and compare it with already existing solutions.

2. The Game Theory Based Power Allocation usage. The aim here is to find out if Game Theory can be adapted practically on off-the-shelf device which would be operating in dynamic wireless environment consisting of third party IEEE 802.11 devices.

3. Multi-band system operation and leveraging of FST system as a way to handle a low performance stations on IEEE 802.11ad. The aim is to experimentally conclude the possibility of practical usage of Multi-band steering engine that is not yet defined in any IEEE 802.11 ad as possibility to increase multi-band system performance as well as less active clients steering. The increase of wireless equipment usage in unlicensed band is growing in multiple countries. The need for higher performance and better QoE increased demand for new type of IEEE 802.11 systems, that would both handle high number of users per single AP as well as efficiently manage the wireless medium. The following study and experimental verification will contribute towards the practical use case for multi-band system.

\subsection{IEEE 802.11 Multi-Poll Experimental Investigation}

The testing setup consists of 83 commercial station devices which are installed at different distances (see Fig. 3.1) and the interconnection scheme in Fig. 3.2, the parameters are listed in Table 3.1. One set of installed devices are placed at $5 \mathrm{~km}$ distance and another set of devices are placed at 200 meters.

The Access Point is Atheros AR9557 chipset-based device with an $17 \mathrm{dBi}$ sectorial antenna and 1000 Base-T ethernet port. The stations are Atheros AR9342 with a $15 \mathrm{dBi}$ directional antenna and 100 Base-T ethernet port. The devices are placed in outdoor environment at different remote locations 61 devices are placed 
at $200-300 \mathrm{~m}$ and 22 devices are placed $5 \mathrm{~km}$ from the AP location. The maximum output power of devices is set between $25 \mathrm{dBm}$ and $30 \mathrm{dBm}$ based on their location. The $5 \mathrm{~km}$ installed stations are using $30 \mathrm{dBm}$ output power and $200 \mathrm{me}-$ ter installed stations are using $25 \mathrm{dBm}$ output power, while AP is using $30 \mathrm{dBm}$ output power.

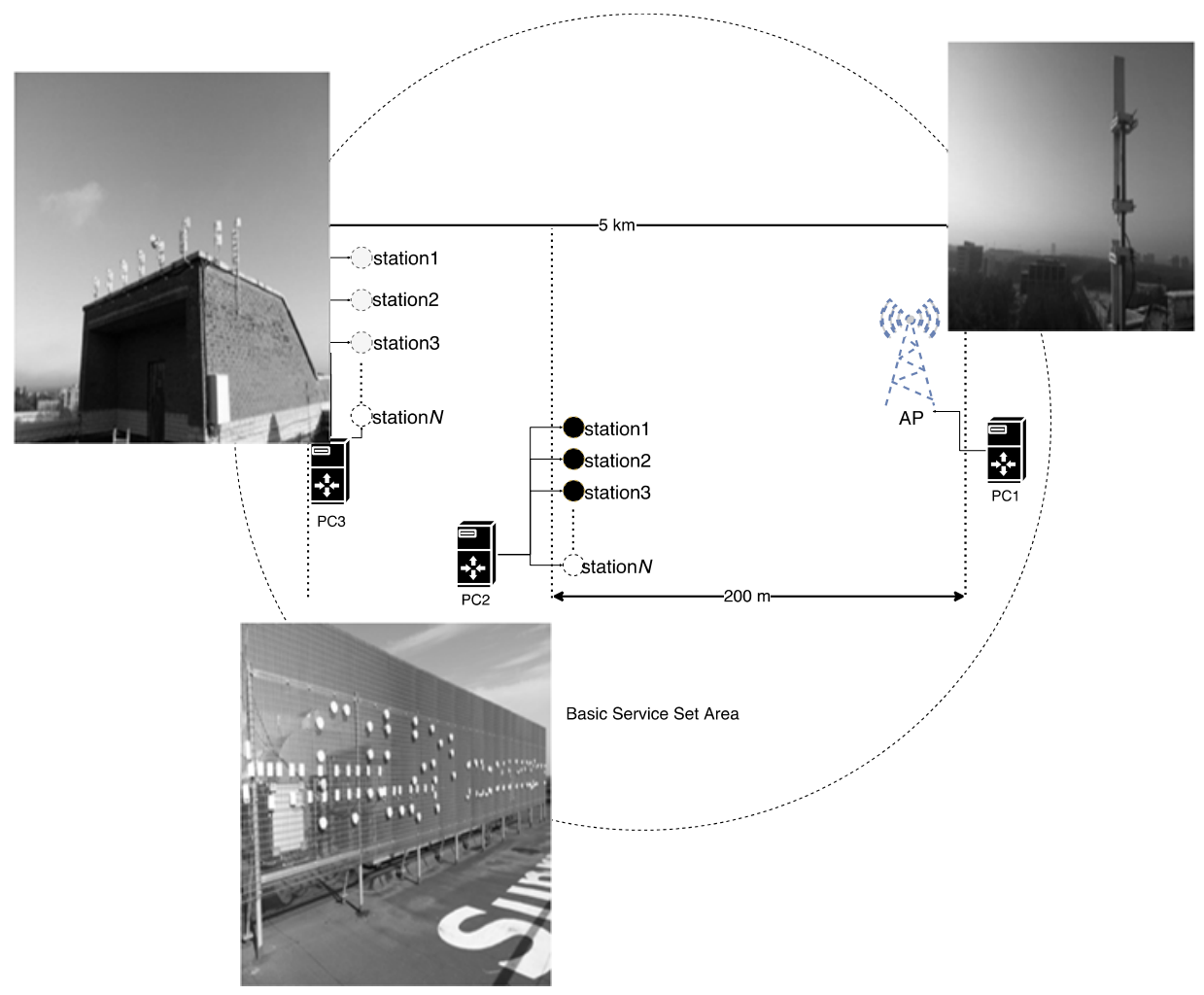

Fig. 3.1. The placement of the devices under experimental test and the installation locations

This is done in order to create a more dynamic environment and a hidden wireless node issue. The selected operational frequency was $5430 \mathrm{MHz}$ and operational channel width is set to $40 \mathrm{MHz}$. The signal level was in range from -45 to $-55 \mathrm{dBm}$ for 200 meter stations and from -60 to $-80 \mathrm{dBm}$ for $5 \mathrm{~km}$ stations. Therefore, the stations can effectively show any disadvantage for protocols when different circumstances occurs. The built setup is used to check both IEEE 802.11 EDCA, IEEE 802.11 PCF (polling-based without Contention Period) as well as proposed Multi-Poll implementation. The reason behind PCF without CP period 
is based on the fact, that State-of-the-art devices implement only polling based approach. Thus, typical State-of-the-art devices either are using TDMA based wireless medium access scheme or the PCF which is based on the Contention Free Period.

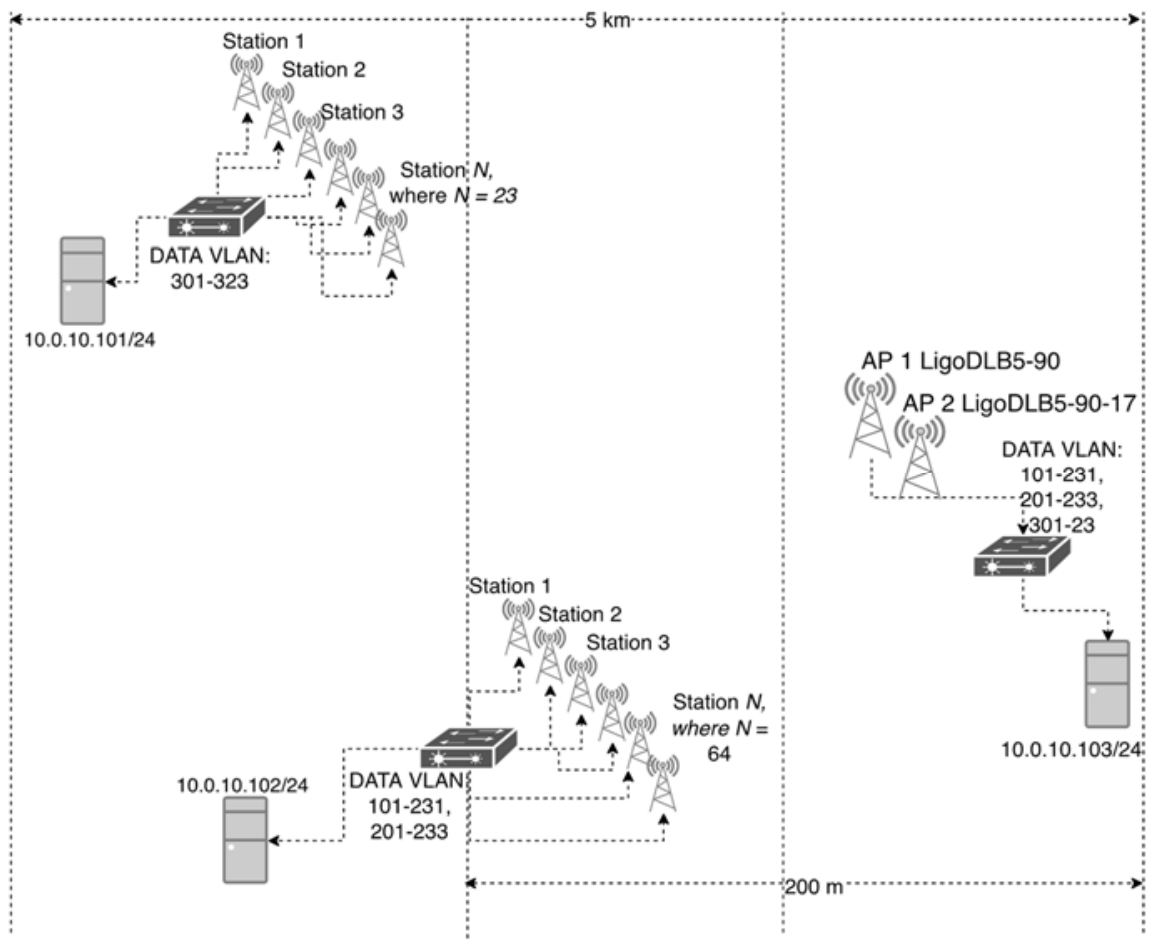

Fig. 3.2. The setup network schematics

Firstly, an experiment will be conducted to evaluate the maximum throughput when number of active clients is being changed. During measurements, a comparison of the test results is performed. The results should reveal if there is any improvement in real setup deployment. The Multi-Poll algorithm merges two DCF and Polling protocols into one - creating a Hybrid Version of both these protocols similar to the IEEE 802.11e (Chen, Lin 2004) standard. On IEEE 802.11 e, only stations with high priority packets are polled, while the others are left to use DCF for wireless medium access. AP contains two lists of connected stations. The stations which are more active will be put to a polling list, and the stations which are less active or idling will use DCF approach to gain access to wireless medium. The stations which have high-priority packets receive capability 
to access wireless medium without waiting for polling tokens. The station activity is measured based on their sent packets per second (PPS) and bytes per second (BPS). The thresholds are controllable on the device and can be changed to have different PPS or BPS values.

Table 3.1. Main parameters of the equipment used for testing

\begin{tabular}{|c|c|}
\hline Parameter & Value \\
\hline IEEE 802.11 standard & $802.11 \mathrm{n}$ \\
\hline Radio mode & MIMO $2 \times 2$ \\
\hline Radio frequency band & $5.150-5.850 \mathrm{GHz}$ \\
\hline Channel size & $80,40 \mathrm{MHz}$ \\
\hline Modulation schemes & $802.11 \mathrm{a} / \mathrm{n}$ : OFDM (64-QAM, 16-QAM, QPSK, BPSK) \\
\hline Data rates & $802.11 \mathrm{n}: 300,270,240,180,120,90,60,30 \mathrm{Mbps}$ \\
\hline Error correction & FEC, Selective ARQ \\
\hline Duplexing scheme & Time division duplex \\
\hline Chipset & AR9342, QCA9557 720 MHz \\
\hline Antenna Gain & 15 and $18 \mathrm{dBi}$ \\
\hline Interface & $10 / 100 / 1000$ Base-T, RJ45 \\
\hline
\end{tabular}

The measurement are performed by using Nepim traffic generator tool. The PCs which are acting as traffic generators are connected to the switch, which connects all the deployed stations. The measurements are done in such way unless otherwise stated:

1. Each measurement is taken for at least 60 seconds, while taking value every $1 \mathrm{~s}$. From these 60 values a single average iteration point is being calculated.

2. The measurement traffic, was TCP single directional or bidirectional depending on the testing scenario. The packet size was 1500 bytes.

3. The co-channel, overlapping and adjacent channel tests were conducted in the same network scheme as displayed above by using two APs in close proximity, which is not more then $50 \mathrm{~cm}$ between, see representative Fig. 3.1 with APs.

4. The power allocation test was done by using two APs and two clients located and different direction, the PCs were used as traffic generator as per scheme in Fig. 3.2. 


\subsubsection{The Short Distance Experimental Measurements}

The following measurement is used to find out the maximum capacity for all the tested IEEE 802.11 protocols and their modifications when the distance between AP and stations is in close proximity, 200 meters. The signals between devices are in range from $-45 \mathrm{dBm}$ to $-55 \mathrm{dBm}$. The data rate is between 270 and $300 \mathrm{Mbps}$ for all the connected stations. It's worth to notice, that setup at $200 \mathrm{~m}$ has not less than 60 stations connected all the time, unless otherwise stated.

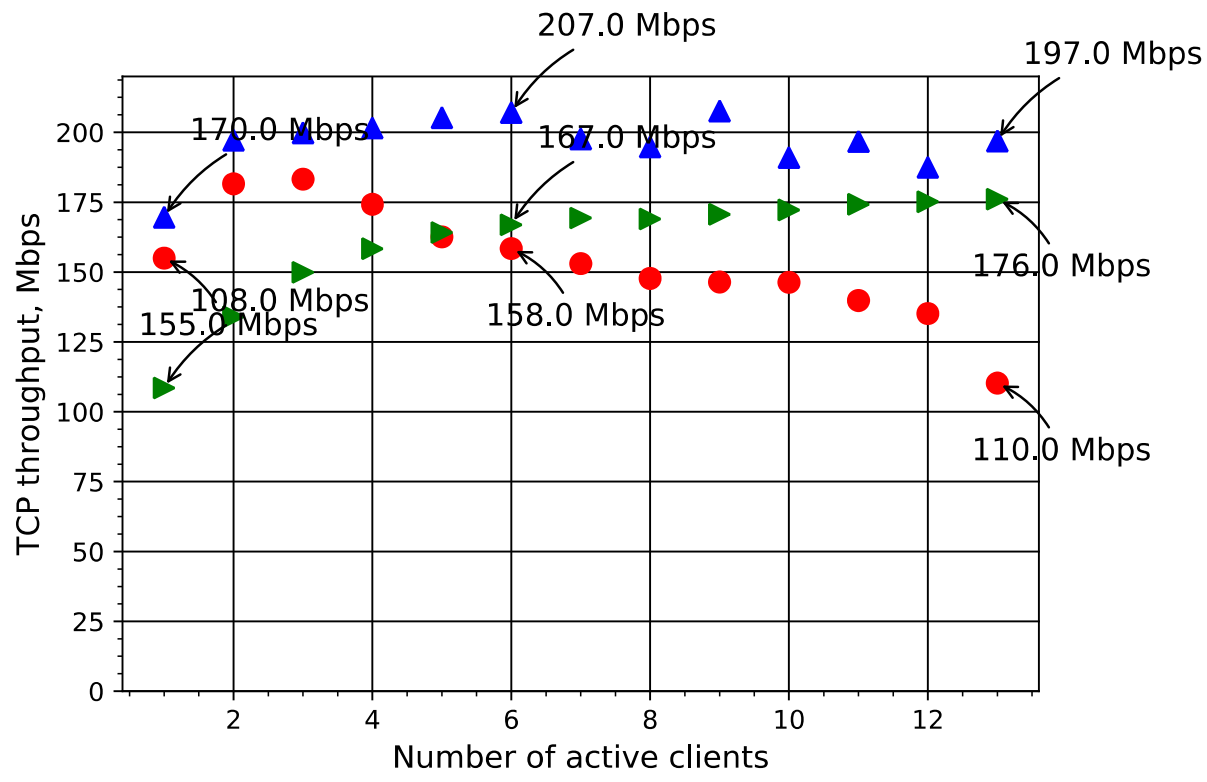

IEEE 802.11 EDCA

- IEEE 802.11 PCF (polling-based without CP)

- Proposed Multi-Poll protocol

Fig. 3.3. Transport control protocol throughput with 13 connected clients

The Fig. 3.3 represents AP performance with 13 clients. The measurement uses TCP traffic to find the highest performance with three protocols: IEEE 802.11 EDCA, IEEE 802.11 PCF without CP and the proposed Multi-Poll. The measurements are done gradually increasing number of active clients. At the beginning of measurement, AP has 13 idling clients, without any data being transfered. After first round of measurement, one active client appears. In this case IEEE 802.11 PCF (polling-based) achieves up to $108 \mathrm{Mbps}$. The IEEE 802.11 EDCA in the exactly same measurement scenario achieves up to $155 \mathrm{Mbps}$. The proposed Multi-Poll implementation achieves up to $170 \mathrm{Mbps}$ as well, when setup 
has only one active station. It can be concluded, that with 1 active station MultiPoll implementation outperforms, the IEEE 802.11 PCF based approach. Continuing the measurement, it can be noticed, that as number of active clients increases the Multi-Poll is able provide stable performance and when active amount of clients reaches 6 on all of the measured protocols, Multi-Poll implementation provides up to $207 \mathrm{Mbps}$, while EDCA provides up to $158 \mathrm{Mbps}$ and PCF based approach with constant CFP provides up to $167 \mathrm{Mbps}$. This gives up to $24 \%$ data throughput increase for Multi-Poll in comparison with PCF based approach without $\mathrm{CP}$ and up to $31 \%$ data throughput increase in comparison with EDCA approach. When all 13 clients are sending, system capacity reaches $110 \mathrm{Mbps}$ for EDCA, $197 \mathrm{Mbps}$ for Multi-Poll and $176 \mathrm{Mbps}$ for PCF. Thus, Multi-Poll provides up to $12 \%$ higher data throughput with all 13 clients being active in comparison with $\mathrm{PCF}$ and up to $79 \%$ higher data throughput in comparison with EDCA approach, when TCP traffic is being sent.
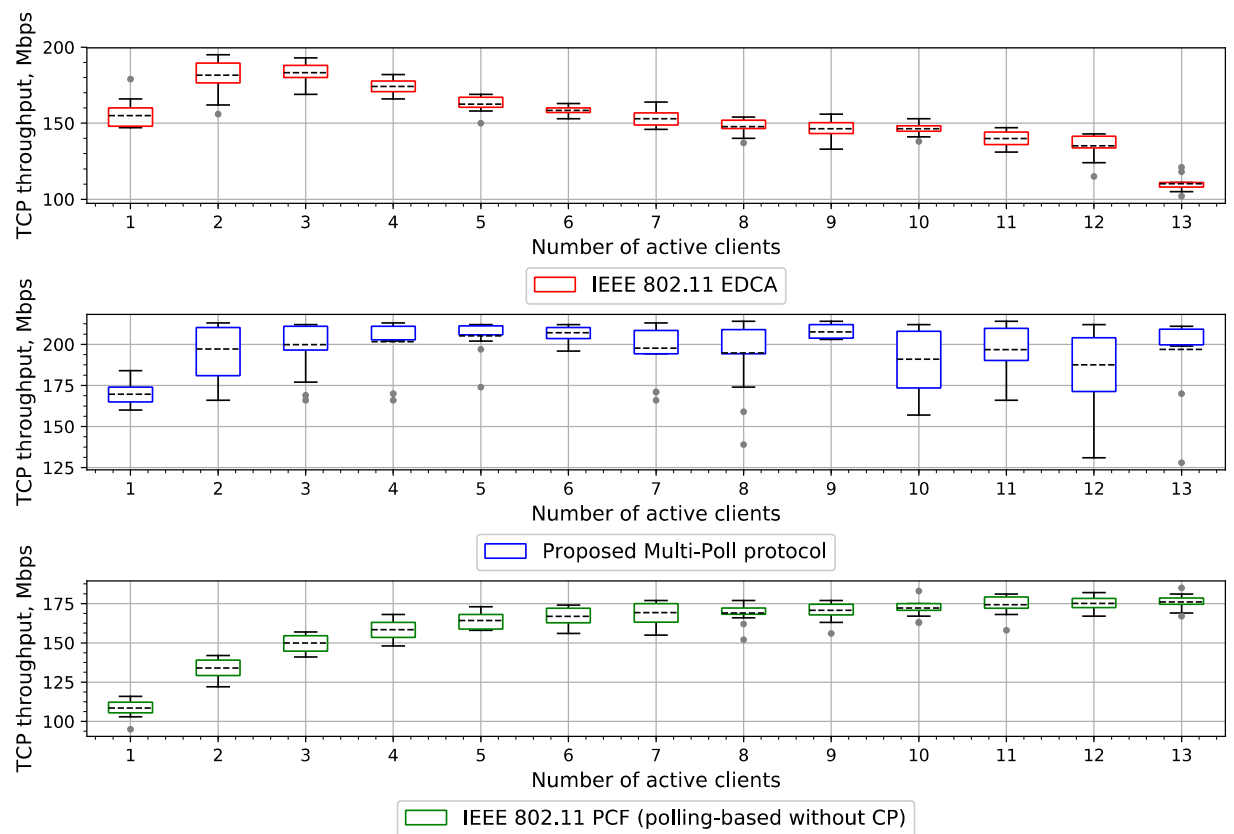

Fig. 3.4. Distribution of measurements with different number of active clients, when total 13 clients are connected

The aggregated TCP measurement results are displayed in Table 3.2. The average system's throughput is depicted in Fig. 3.4. as a boxplot of the average throughput against different number of active stations within the network. The active client number is increased with each measurement and performance of the 
suggested protocols is measured by running TCP stream generated by Nepim utility. The data set includes 10 measurements with every new client. When one active client out of 13 is sending bidirectional TCP stream, it can reach from $148 \mathrm{Mbps}$ up to $166 \mathrm{Mbps}$ for IEEE 802.11 EDCA. The proposed Multi-poll protocol can reach at the same time from $160 \mathrm{Mbps}$ up to $185 \mathrm{Mbps}$ and PCF without $\mathrm{CP}$ can reach from $100 \mathrm{Mbps}$ up to $115 \mathrm{Mbps}$. When all 13 clients are active, proposed Multi-Poll reaches from $200 \mathrm{Mbps}$ up to $210 \mathrm{Mbps}$, while IEEE 802.11 EDCA reaches from $100 \mathrm{Mbps}$ up to $110 \mathrm{Mbps}$, while PCF (polling based without Contention Period) in this case reaches from $170 \mathrm{Mbps}$ up to $180 \mathrm{Mbps}$.

Table 3.2. Aggregated TCP Throughput with different protocols

\begin{tabular}{|c|c|c|c|}
\hline $\begin{array}{c}\text { Number of Active } \\
\text { Clients }\end{array}$ & Multi-Poll, Mbps & $\begin{array}{c}\text { IEEE 802.11 } \\
\text { EDCA, Mbps }\end{array}$ & $\begin{array}{c}\text { IEEE 802.11 PCF } \\
\text { (polling based } \\
\text { without CP) } \\
\text { protocol, Mbps }\end{array}$ \\
\hline 1 & 169 & 155 & 108 \\
\hline 2 & 197 & 181 & 134 \\
\hline 3 & 199 & 183 & 150 \\
\hline 4 & 201 & 174 & 158 \\
\hline 5 & 205 & 162 & 164 \\
\hline 6 & 207 & 158 & 166 \\
\hline 7 & 197 & 153 & 169 \\
\hline 8 & 194 & 147 & 169 \\
\hline 9 & 207 & 146 & 170 \\
\hline 10 & 191 & 146 & 172 \\
\hline 11 & 196 & 139 & 174 \\
\hline 12 & 187 & 135 & 175 \\
\hline 13 & 197 & 110 & 176 \\
\hline & & & \\
\hline
\end{tabular}

As further designed test's aim was to verify how the performance is impacted when the number of connected stations increases. Random set of stations were selected out of 60 located 200 meters from the Access Point. After the selection of random station, TCP traffic bidirectional was started for each of the stations in sequence. The measurement for each active client took 90 seconds and average of 90 seconds was being calculated for each individual client. The signal level for active clients is in range from $-40 \mathrm{dBm}$ up to $-55 \mathrm{dBm}$. The data rates are in range from $270 \mathrm{Mbps}$ up to $300 \mathrm{Mbps}$. The traffic type is TCP with packet size of 1500 bytes. 


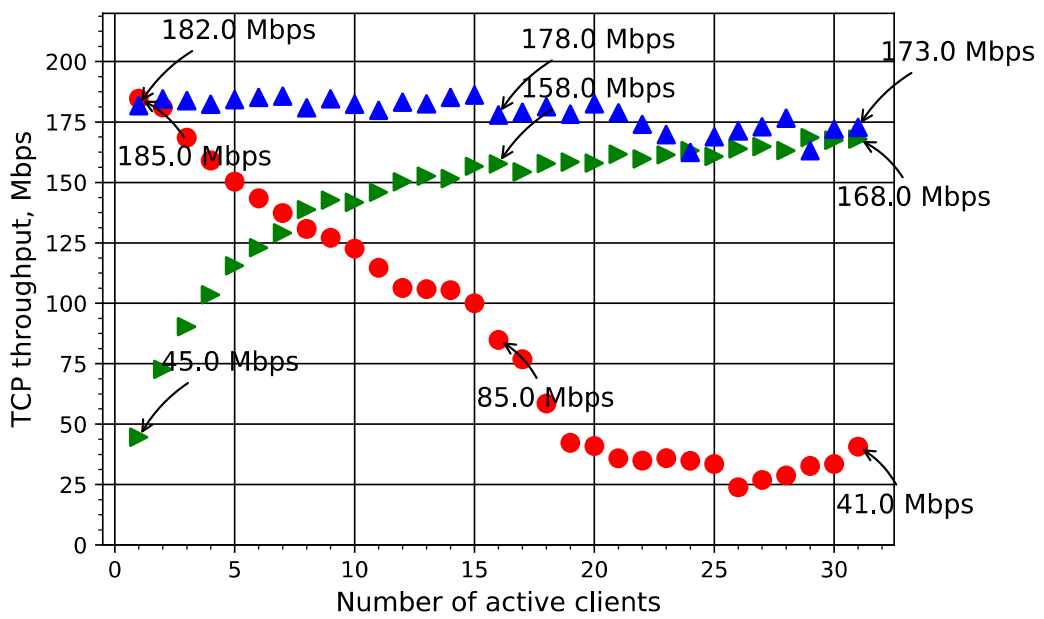

IEEE 802.11 EDCA

A Proposed Multi-Poll protocol

- IEEE 802.11 PCF (polling-based without CP)

Fig. 3.5. Transport control protocol throughput with 31 connected clients
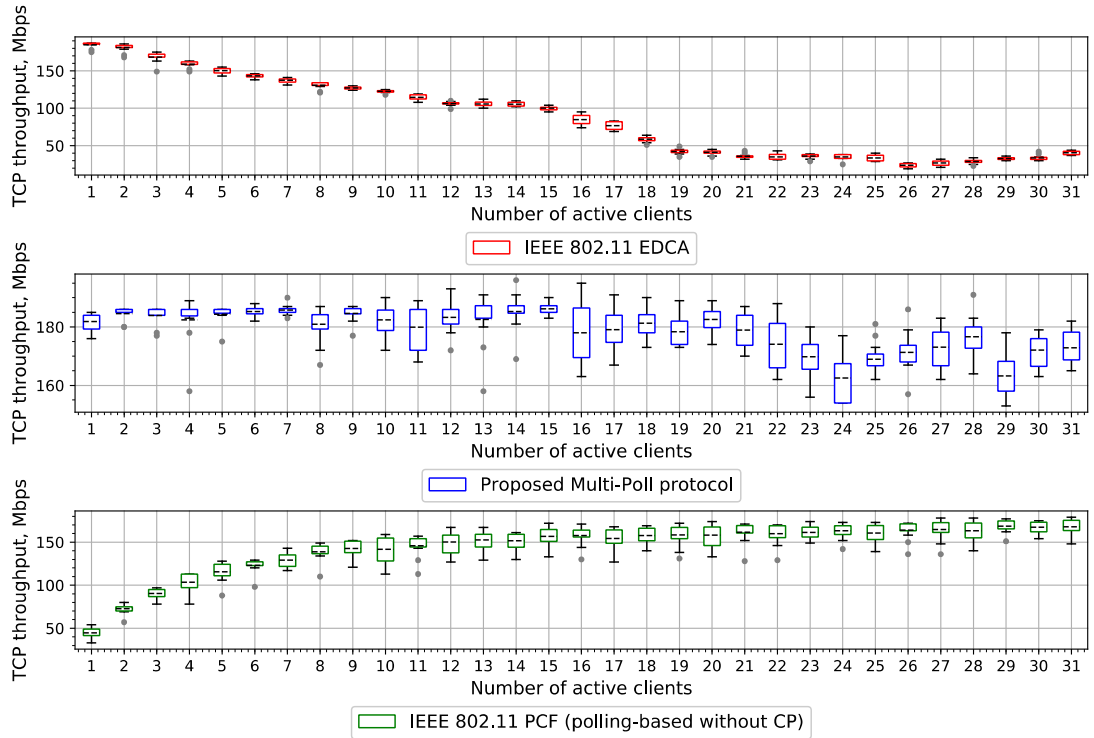

Fig. 3.6. Distribution of measurements with different number of active clients, when total 31 clients are connected 
The impact is measured while running traffic under different circumstances, which include the different number of active clients in Fig. 3.5. Even with increasing number of connected stations, Multi-Poll is able to handle both high and low number of active clients. When setup has 16 active clients, the Multi-Poll reaches $178 \mathrm{Mbps}$ throughput, IEEE 802.11 PCF based approach without CP reaches $158 \mathrm{Mbps}$ and IEEE 802.11 EDCA reaches $85 \mathrm{Mbps}$

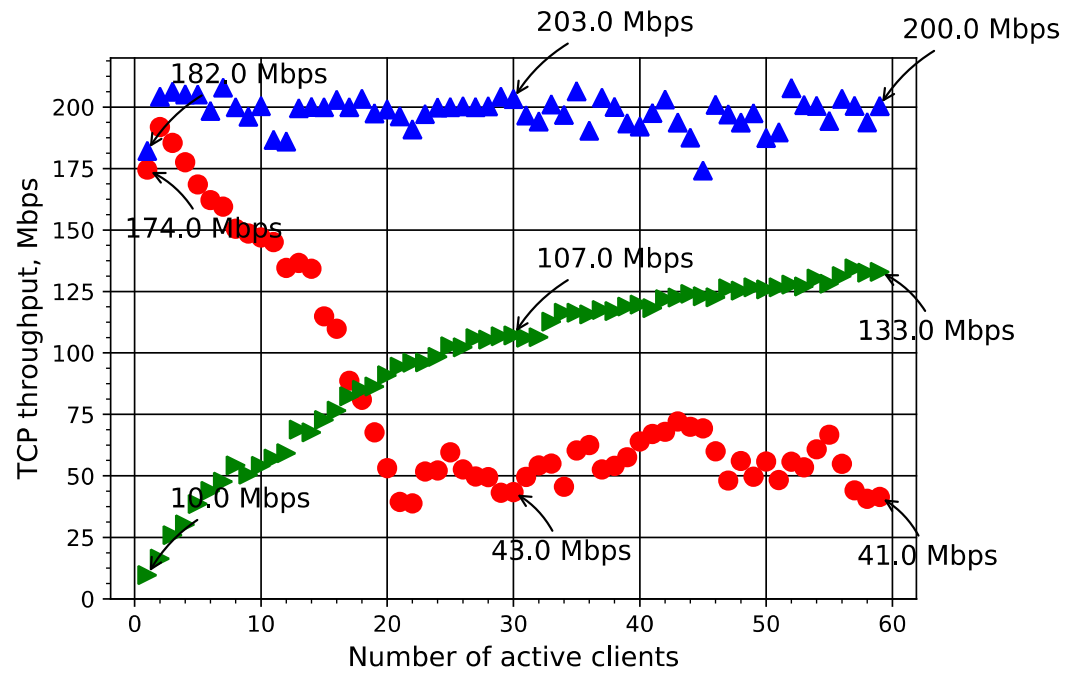

IEEE 802.11 EDCA

- IEEE 802.11 PCF (polling-based without CP)

A Proposed Multi-Poll protocol

Fig. 3.7. Throughput under different protocols with 60 clients

In the next experimental measurement AP has 60 clients connected. All the connected clients in the initial stage are inactive - no data is being transferred. After uptime for all stations reaches more than 3 minutes, the next measurement attempt is being performed. The TCP stream is being generated for every associated client in sequence and the results are depicted in the Fig. 3.7. As the number of inactive clients on AP increases, it can be noticed, that PCF without CP based protocol performance decreases if the number of active clients is low. This can be explained, that PCF in Contention Free Period performs polling of every connected client, even if there isn't any actual data. This causes additional airtime consumption and thus increases the round trip time for the client which is active. This leads to the lower initial performance, when number of active clients is below 10. As can be noticed, when there is only 1 active client the PCF without CP can reach up to $10 \mathrm{Mbps}$, the EDCA based approach reaches up to $174 \mathrm{Mbps}$ and 
Multi-Poll based approach reaches up to $182 \mathrm{Mbps}$. The Multi-Poll approach same as EDCA under low activity stage uses EDCA wireless medium access scheme, thus all the inactive stations are contending for the wireless medium under EDCA rules, therefore the amount of polled stations is low and initial performance is approximately 18 times higher than the PCF without $\mathrm{CP}$. When half of the clients are idle, the Multi-Poll achieves $203 \mathrm{Mbps}$, in meantime EDCA achieves $43 \mathrm{Mbps}$ and PCF polling-based approach without CP achieves $107 \mathrm{Mbps}$. Thus, Multi-poll performance increase of data throughput is up to $372 \%$ in comparison with EDCA based approach and up to $89 \%$ in comparison with PCF based approach. The same measurement was continued and when all 60 clients are sending data, the Multipoll is able to reach $200 \mathrm{Mbps}$, while PCF polling based without CP and EDCA can reach only $133 \mathrm{Mbps}$ and $41 \mathrm{Mbps}$ accordingly. Thus, Multi-poll with all 60 active clients is able to increase system performance up to $50 \%$ in comparison with PCF polling based approach and up to $388 \%$ in comparison with IEEE 802.11 EDCA. The boxplot in Fig. 3.8 represents the distribution of the throughput results based on the number of active stations. As can be noticed, during different data measurement sets the Multi-Poll has some variance.

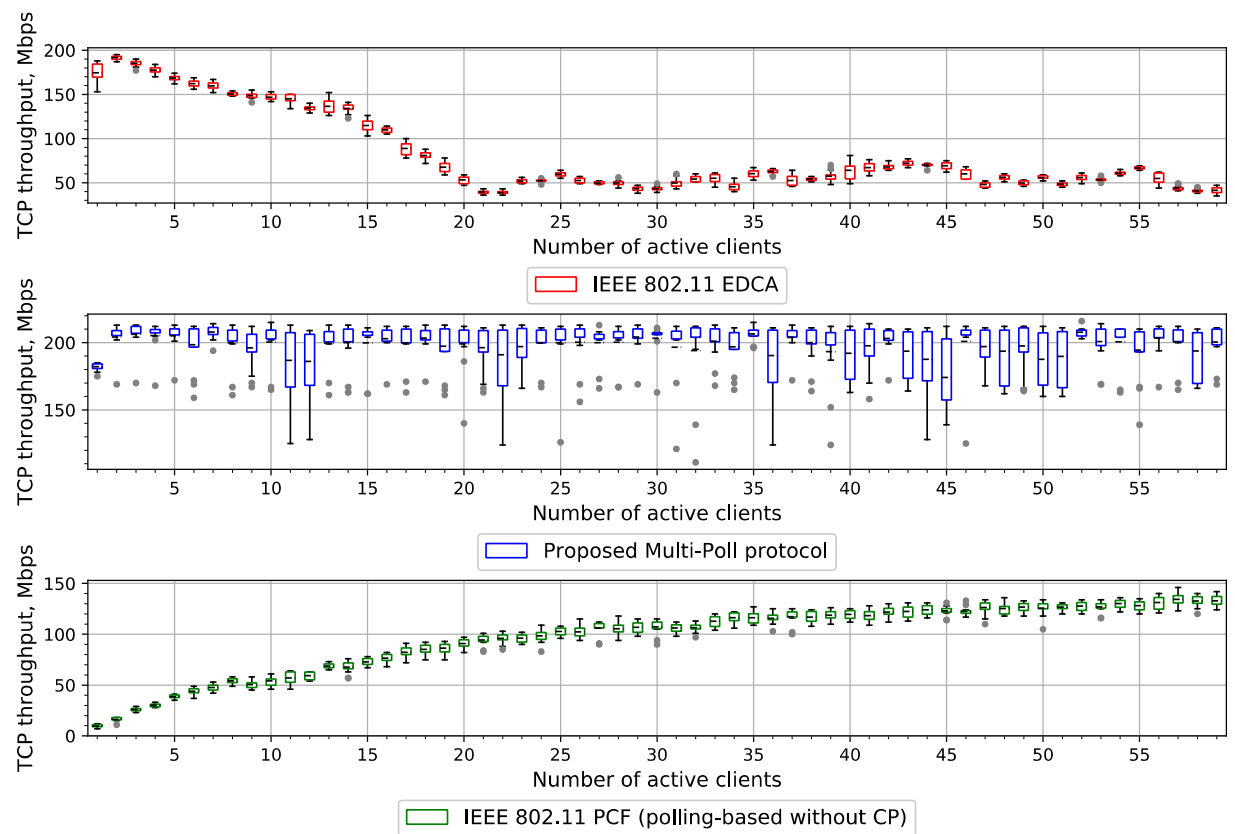

Fig. 3.8. Distribution of measurements with different number of active clients, when total 60 clients are connected 
In order to evaluate the fairness phenomenon a fairness calculations have been performed for each of defined protocols. The Jains equation in (3.1) (Jain et al. 1984 ) provides a way to evaluate how equally the access to wireless medium is split between connected stations of the wireless system, which contains $n$ stations. The Jains fairness index is given by expression:

$$
J=\frac{\left(\sum_{i=1}^{n} b_{i}\right)^{2}}{n \times \sum_{i=1}^{n} b_{i}^{2}},
$$

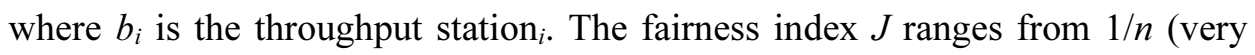
unfair) to 1 (complete fairness).

Table 3.3. Aggregated Throughput and Jains Fairness index with different protocols

\begin{tabular}{|l|l|l|l|}
\hline \multirow{2}{*}{ Protocol } & Data stream direction & Throughput, Mbps & $\begin{array}{l}\text { Jain's } \\
\text { Index }\end{array}$ \\
\hline \multirow{4}{*}{ Multi-Poll } & Bidirectional & 3.6 & 0.769 \\
\cline { 2 - 4 } & Downlink & 1.91 & 0.607 \\
\cline { 2 - 4 } & Uplink & 1.69 & 0.73 \\
\hline \multirow{3}{*}{ EDCA } & Bidirectional & 2.33 & 0.393 \\
\cline { 2 - 4 } & Downlink & 0.15 & 0.358 \\
\cline { 2 - 4 } & Uplink & 2.18 & 0.392 \\
\hline \multirow{3}{*}{$\begin{array}{l}\text { PCF polling-based } \\
\text { without CP }\end{array}$} & Bidirectional & 2.61 & 0.986 \\
\cline { 2 - 4 } & Downlink & 0.74 & 0.944 \\
\cline { 2 - 4 } & Uplink & 1.86 & 0.978 \\
\hline
\end{tabular}

The Table 3.3. includes average throughput and fairness parameters for different protocols. The highest average throughput in Downlink and Aggregated direction is achieved by using Multi-Poll approach. The EDCA approach in Downlink direction suffers from collisions between stations, since each of the connected station tries to gain access to WM, therefore the EDCA Downlink is the lowest between measured protocols - this is noticeable based on the fairness index as well. Additionally, from the Jains fairness index it can be noticed, that Multi-Poll does not guarantee the fairness between wireless medium access, this is the reason because of the shared queue at AP side for all the connected stations. The reason for this is that all same AC data packets are placed in same buffer. The principal of queue operates under FIFO, therefore some stations takes more queue then the other, which clearly displays the impact to the fairness, this is especially noticeable due to the TCP traffic used for measurements. However, even with less 
fair access Multi-Poll can still provide 4 times higher throughput then the other benchmarked protocols.

\subsubsection{The Hidden Node Scenario Experimental Measurements}

The hidden wireless node scenario is widely discussed in the scientific articles that tries to increase efficiency of IEEE 802.11 networks. The previous chapters discussed various approaches which can improve the efficiency of wireless system when it includes several hidden wireless nodes. In order to conclude the statements discussed in previous chapters a test case was designed, which purpose is to evaluate the impact for performance when in BSS operates several stations, which are operating at different distances. In this case 3 stations at $5 \mathrm{~km}$ are used to cause the effect of hidden node against the station which operates at 200 meters. The first measurements were started by running Nepim TCP stream in downlink direction, that is from AP to all the connected stations.

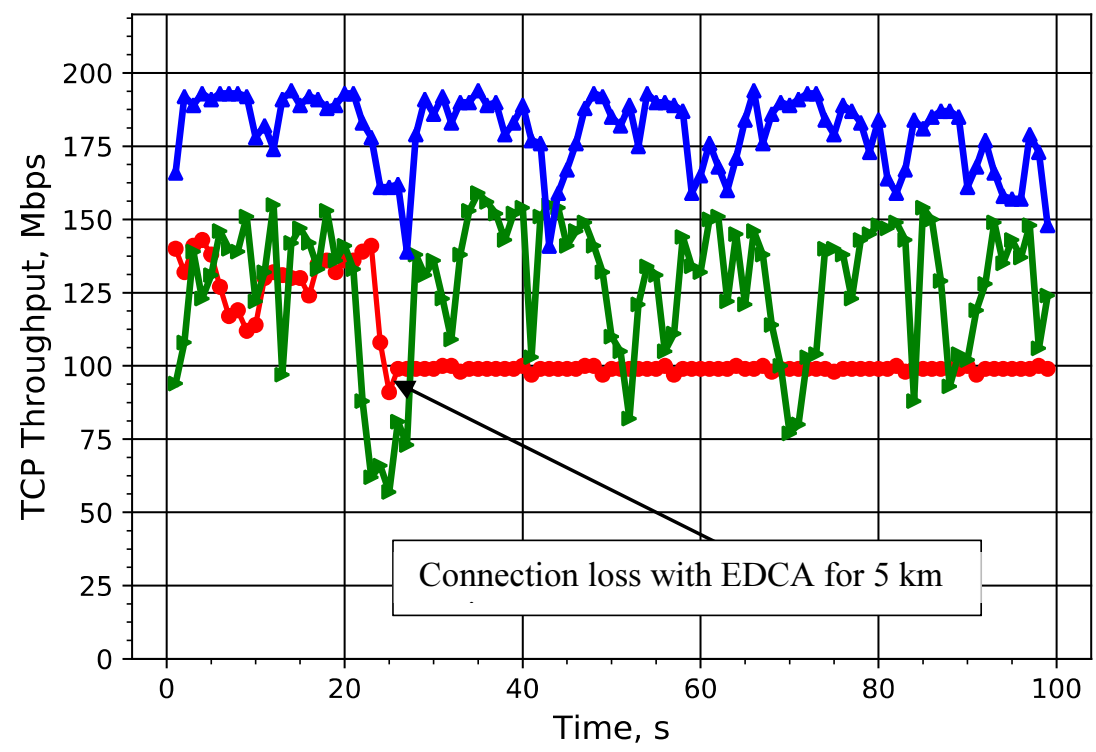

$\rightarrow$ IEEE 802.11 EDCA
$\rightarrow$ Modified IEEE 802.11 PCF based protocol

Fig. 3.9. Transport Control Protocol downlink performance

In Fig. 3.9. the downlink traffic was generated from AP to 4 stations, while 3 of them were at $5 \mathrm{~km}$ distance and 1 at $200 \mathrm{~m}$ distance. As can be noticed both EDCA and PCF without CP based approaches provides unstable throughput. The 
instability can be explained by following statement. The environment in which the measurements are prepared are shared with third party IEEE 802.11 wireless devices, additionally the stations which are operating at $5 \mathrm{~km}$, have a signal level in range from -63 to $-70 \mathrm{dBm}$ therefore, stations located at $5 \mathrm{~km}$ retries a lot more and takes available airtime for retransmissions (Zhang et al. 2012), excessive retries results in connection loss, which can also be seen from Fig. 3.9.

Finally, the collisions between hidden node under EDCA protocol causes lost frames, which impacts the TCP traffic stream stability as well as rate control algorithm being used on the device. While, Multi-Poll shows better results when compared to EDCA and PCF in this downlink measurement it can be still noticed that TCP stream is changing over measured time interval.

The Table 3.4 displays the performance for each of the stations which were used in the measurement as can be seen the highest aggregated throughput is reached by using Multi-Poll protocol.

This again can be explained that stations which are capable to handle higher speed, fill the data queue more quickly than the stations with low signal levels. Therefore, the total system capacity is less impacted by the clients which retries a lot. The best fairness index is reached by PCF (polling based without $\mathrm{CP}$ ) approach, however the aggregated system capacity is the lowest between three measured protocols.

As mentioned before the uplink direction is typically the cause of the collisions and low performance in IEEE 802.11 systems.

Table 3.4. Downlink Performance with different protocols

\begin{tabular}{|l|l|l|l|l|l|l|}
\hline Protocol & $\begin{array}{c}\text { Station1 } \\
200 \mathrm{~m}, \\
\text { Mbps }\end{array}$ & $\begin{array}{c}\text { Station2 } \\
5 \mathrm{~km}, \\
\mathrm{Mbps}\end{array}$ & $\begin{array}{c}\text { Station3 } \\
5 \mathrm{~km}, \\
\mathrm{Mbps}\end{array}$ & $\begin{array}{c}\text { Station4 } \\
5 \mathrm{~km}, \\
\mathrm{Mbps}\end{array}$ & $\begin{array}{c}\text { Aggregated, } \\
\text { Mbps }\end{array}$ & $\begin{array}{l}\text { Jain's } \\
\text { Index }\end{array}$ \\
\hline EDCA & 92 & 5.5 & 5 & 5.7 & 108.2 & 0.343 \\
\hline $\begin{array}{l}\text { PCF with- } \\
\text { out CP }\end{array}$ & 58 & 18.8 & 20.2 & 18.5 & 115.5 & 0.746 \\
\hline Multi-Poll & 88 & 31.2 & 29.4 & 27.6 & 176.2 & 0.750 \\
\hline
\end{tabular}

This is especially noticeable in outdoor based installation where stations can't hear each other. As can be seen from Fig. 3.10. the uplink for Multi-Poll is stable compared to EDCA and average $155 \mathrm{Mbps}$ can be achieved, while PCF and EDCA are achieving only between 70 and $94 \mathrm{Mbps}$. However, the graph does not represent how the actual traffic is splitted between the connected stations. Based on the results it can be concluded, that EDCA can't perform well in hidden node scenario without impacting overall system performance. 

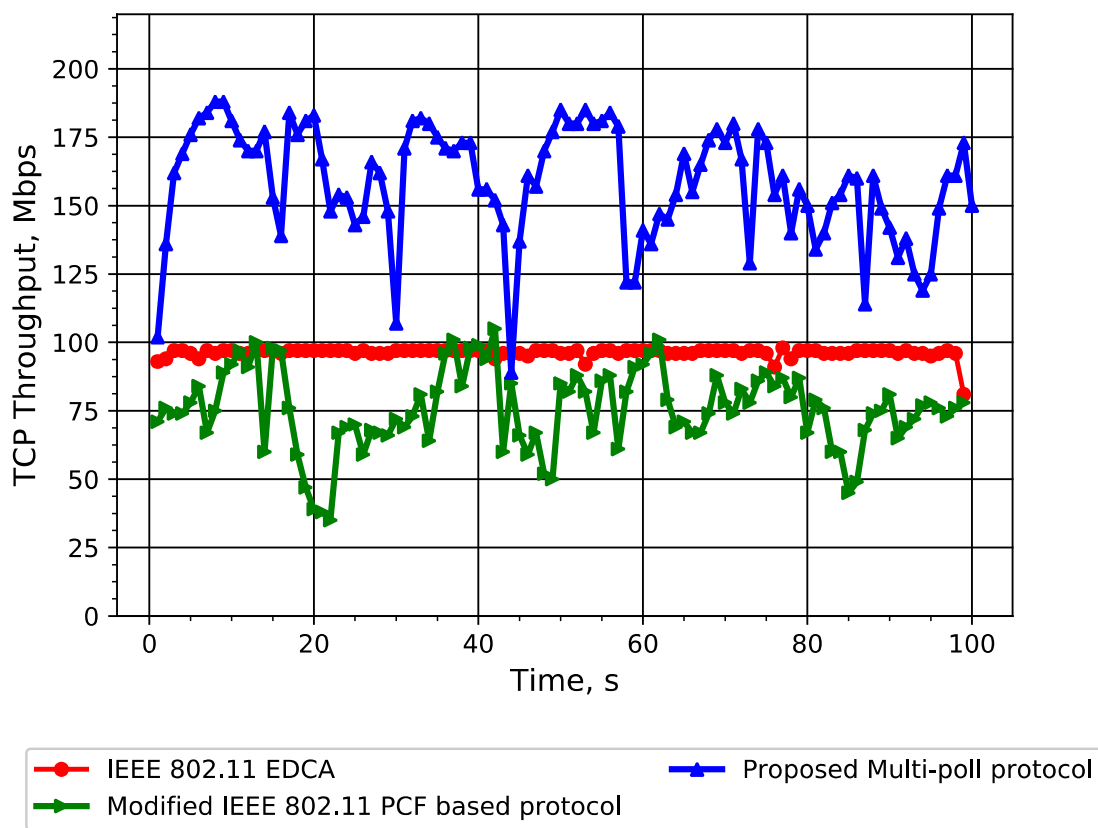

Fig. 3.10. Transport Control Protocol uplink performance

The Table 3.5 represents the performance for each of the stations. EDCA is underperforming in uplink traffic test, the throughput for stations located at $5 \mathrm{~km}$ is between $0.1 \mathrm{Mbps}$ and $0.2 \mathrm{Mbps}$ and fairness index is 0.253 . When multiple stations try to send their data at the same time, the back off mechanism is activated for each of these stations. Any packets loss results in auto rate decrease on the device. At the end, total system capacity is lower compared to standard polling solution or the Multi-Poll protocol.

Table 3.5. Uplink Performance with different protocols

\begin{tabular}{|c|c|c|c|c|c|c|}
\hline Protocol & $\begin{array}{c}\text { Station1 } \\
200 \mathrm{~m}, \\
\text { Mbps }\end{array}$ & $\begin{array}{c}\text { Station2 } \\
5 \mathrm{~km}, \\
\text { Mbps }\end{array}$ & $\begin{array}{c}\text { Station3 } \\
5 \mathrm{~km}, \\
\text { Mbps }\end{array}$ & $\begin{array}{c}\text { Station4 } \\
5 \mathrm{~km}, \\
\text { Mbps }\end{array}$ & $\begin{array}{c}\text { Aggregated, } \\
\text { Mbps }\end{array}$ & $\begin{array}{c}\text { Jain's } \\
\text { Index }\end{array}$ \\
\hline EDCA & 93 & 0.2 & 0.1 & 0.2 & 93.5 & 0.253 \\
\hline $\begin{array}{c}\text { PCF } \\
\text { without } \\
\text { CP }\end{array}$ & 45 & 8.3 & 9.7 & 7.8 & 70.8 & 0.557 \\
\hline $\begin{array}{c}\text { Multi- } \\
\text { Poll }\end{array}$ & 84.8 & 35.7 & 24.1 & 10.4 & 155 & 0.656 \\
\hline
\end{tabular}


The Multi-Poll protocol in both cases provides the highest aggregated throughput. The fairness among the clients in Multi-Poll protocol depends highly depends on the connected station capabilities, if signal and modulation is low for connected station, the queue by such station will be underutilized and the station with higher capabilities will be able to take full advantage of the queue.

\subsubsection{The Round Trip Time Experimental Measurements}

In the next test, an overview of the real-time response under load conditions will be conducted. This is done to evaluate the round-trip-time (RTT) when there is a different amount of traffic generated over the connected stations and some of them are in idle state.

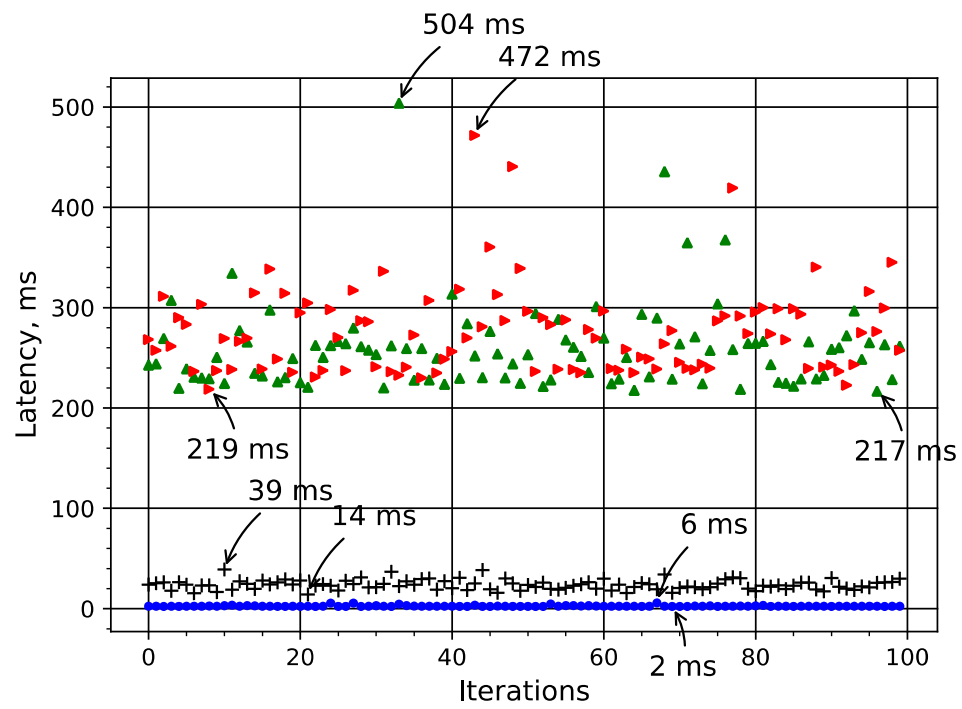

- PCF polling based without CP latency in idle state - Multi-Poll latency in idle state

- PCF polling based without CP latency in active state + Multi-Poll latency in active state

Fig. 3.11. User's latency under standard polling and multi-polling conditions. When total of 80 clients are connected to Access Point

Fig. 3.11 shows the results when only one active client exists out of 80 connected. The active client is generating $90 \mathrm{Mbps}$ throughput, while the one which was randomly selected for RTT measurements was idling. The Multi-poll result provides more steady latency compared to the standard round robin based polling solution, which is being used by PCF (without CP) approach. The idling clients by default re-use the standard EDCA approach, while the more active ones are 
moved to round robin scheduler and the access is controlled by using token mechanism. The low PPS and BPS applications such as voice or Internet Control Message Protocol (ICMP) will have lower latency compared to standard polling. The smaller active scheduler list provides better latency to the active stations and results in higher TCP performance, which is latency and packet loss sensitive. On average PCF polling based without CP in idle state latency approximately is $258 \mathrm{~ms}$, while in the same situation Multi-Poll latency is $2.6 \mathrm{~ms}$. In active state, when one randomly selected station is transferring data the latency is approximately $276 \mathrm{~ms}$ for PCF polling based without $\mathrm{CP}$ solution and approximately $23 \mathrm{~ms}$ for Multi-Poll solution, this is 12 times lower packet latency in comparison with PCF polling based approach.

\subsubsection{Co-existence Between Legacy and Multi-Poll Protocol}

Since Multi-Poll approach will be coexisting with already deployed wireless solutions, the next test includes assumption that third party device operating under standard IEEE 802.11 EDCA rules shares the wireless medium with the MultiPoll Protocol.

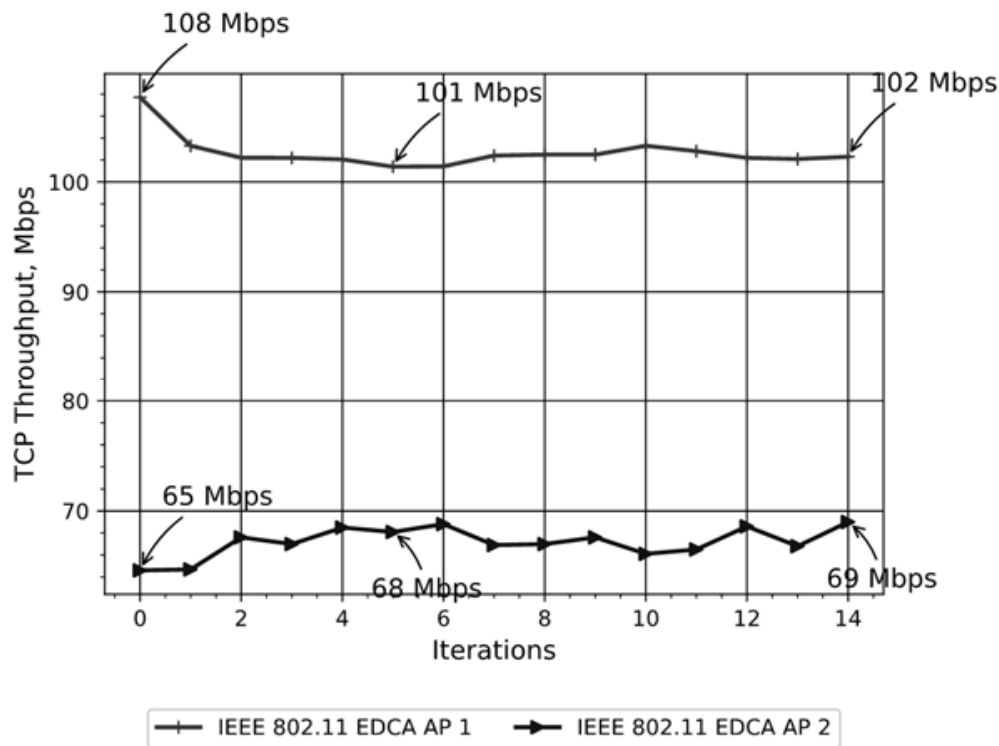

Fig. 3.12. IEEE 802.11 Enhanced Distributed Channel Access performance in co-channel operation

The aim of such test is to evaluate if the wireless medium can be shared between the legacy and the new wireless medium access protocols. The test was performed using the same devices as in previous section, firstly two IEEE 802.11 
EDCA APs were deployed with clients connected to each of the AP and a performance measurement was issued. Both of the APs were located in close proximity and therefore an impact of overlapping, co-channel scenarios can be tested under different connection parameters. The same tests were performed when both APs were operating in EDCA and when one of the AP is operating in proposed MultiPoll protocol. Fig. 3.12. displays performance of IEEE 802.11 EDCA standard when two APs and clients operate in close proximity at the same time. In this case a bidirectional throughput is being generated for both of these APs.

The measurement was performed by using Nepim packet generator. As can be seen from Figure above, the second AP2 has lower performance results, this allows to conclude that IEEE 802.11 EDCA standard isn't always operating under fair conditions when several APs gain the same amount of time for wireless medium access. While the first AP can achieve over $100 \mathrm{Mbps}$, the second one reaches only up to $70 \mathrm{Mbps}$. Each iteration last for $20 \mathrm{~s}$, during which an average of all measured values is estimated.

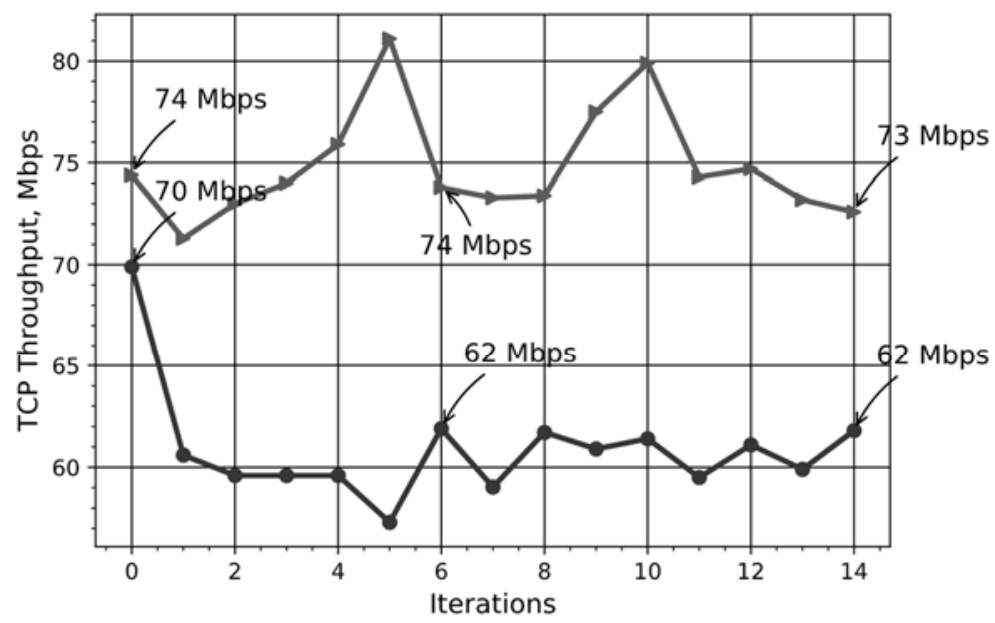

IEEE 802.11 EDCA $\multimap$ Proposed Multi-Poll protocol

Fig. 3.13. IEEE 802.11 Enhanced Distributed Channel Access and Multi-Poll performance in co-channel operation

Under the same conditions, the second attempt to measure was completed. The Fig. 3.13 displays the fairness between Multi-Poll and EDCA based legacy protocol, the result shares the same pattern, that one of the protocols displays lower capacity due to unequal airtime sharing between the APs. Nevertheless, both of these protocols are able to operate in co-channel from which it can be 
concluded, that Multi-Poll is a wireless medium aware protocol and does not occupy the available wireless medium completely unlike the TDMA based approaches, which were evaluated before.

\subsubsection{Time Division Multiple Access and Multi-Poll Comparison}

A comparison performance experiment was conducted by using a TDMA based equipment. TDMA uses a fixed timeslot for transmission and reception, in following case a TDMA was set to use $10 \mathrm{~ms}$ timeslot with different uplink and downlink ratios. The setup consists of AP IEEE 802.11 ac with TDMA capability (QCA9557) and 3 clients (AR724X) IEEE 802.11n with TDMA capability, which are placed in close proximity, see Fig. 3.14. The signal levels were between $45 \mathrm{dBm}$ and $-55 \mathrm{dBm}$. Transmit power due to close proximity is set to $-4 \mathrm{dBm}$. Omnidirectional antennas are used on clients.

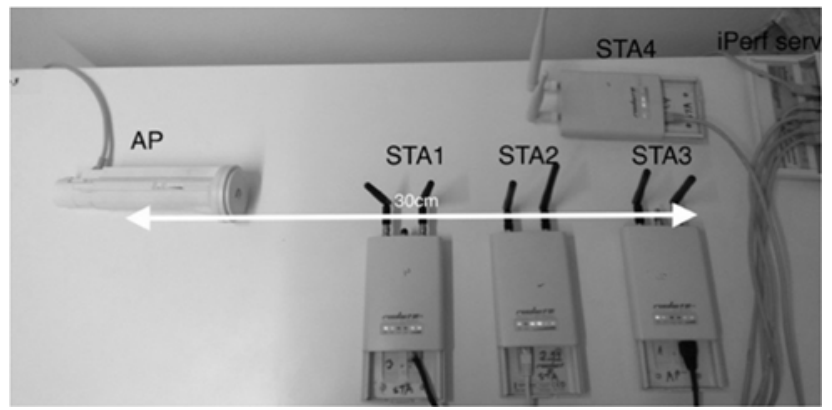

Fig. 3.14. Time division multiple access test setup

The measurement was performed by running Nepim bidirectional tests over 3 clients. The selected frequency was $5745 \mathrm{MHz}$ with channel width of $40 \mathrm{MHz}$. In order to verify TDMA operation under different cases, several measurements were performed by adjusting both uplink and downlink ratio with fixed timeslot size of $10 \mathrm{~ms}$. The given results are depicted in Fig. 3.15.

The Fig. 3.15 indicates the performance difference as can be noticed the Multi-Poll approach which does not limit transmission time for each station is able to achieve higher performance figure. On average total aggregated system capacity reaches approximately up to $217 \mathrm{Mbps}$ for Multi-Poll protocol, while fixed frame TDMA reaches approximately up to $125 \mathrm{Mbps}$ with fixed frame $(75 / 25$ downlink and uplink ratio) and with variable TDMA frame size reaches up to $141 \mathrm{Mbps}$. Therefore, non-restrictive transmission in time (Multi-Poll) provides up to $53 \%$ higher throughput when the transmission time is not restricted per station in comparison with variable TDMA frame size. Thus, it can be concluded, 
that when systems has relatively low number of stations Multi-Poll outperforms the TDMA based solution.
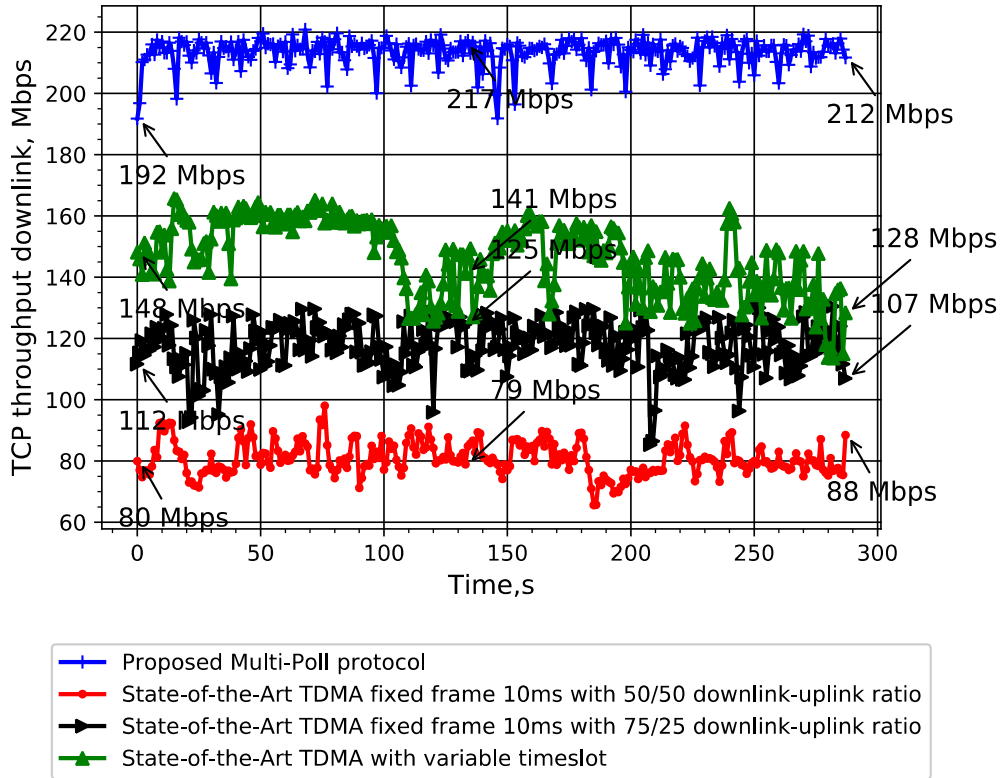

Fig. 3.15. Time Division Multiple Access and Multi-Poll performance with 3 active clients when downlink traffic is being sent
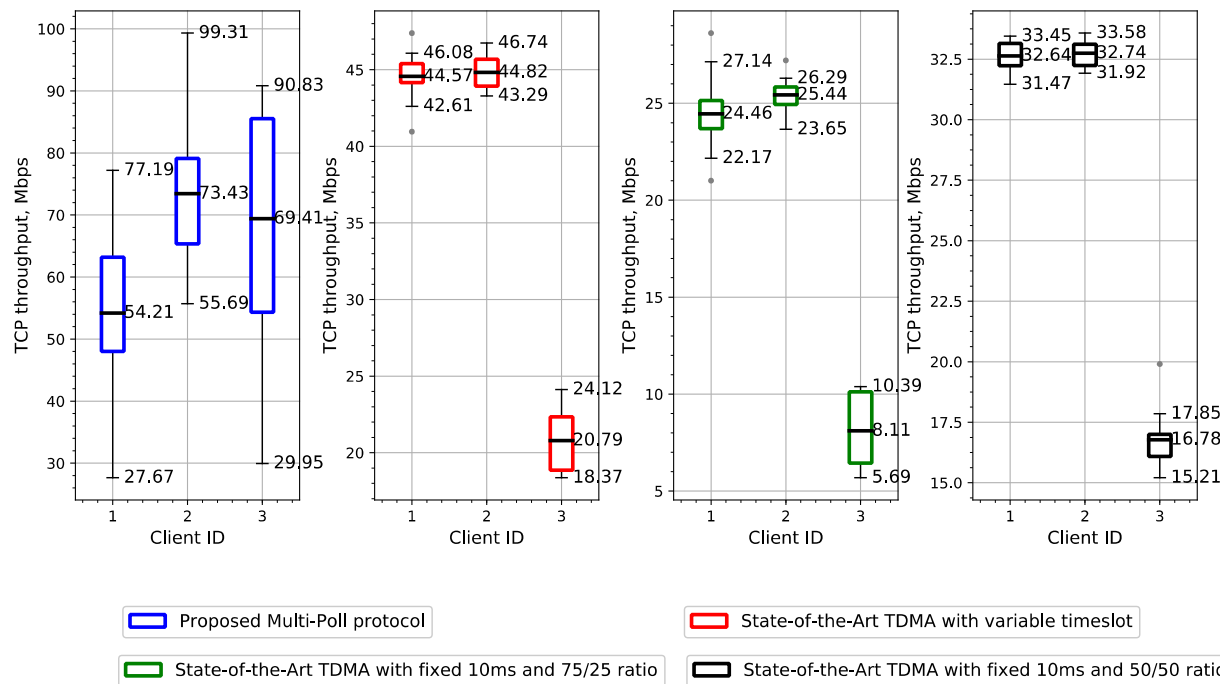

$\square$ State-of-the-Art TDMA with variable timeslot

State-of-the-Art TDMA with fixed $10 \mathrm{~ms}$ and $75 / 25$ ratio

State-of-the-Art TDMA with fixed $10 \mathrm{~ms}$ and 50/50 ratio

Fig. 3.16. Average client's performance when all 3 clients are active and bidirectional traffic is being sent 
The boxplot graph in Fig. 3.16 represents the average user's throughput, when all 3 clients are actively passing data. As can be noticed from the graph, the highest capacity is reached by the clients, that are using Multi-Poll based approach instead of fixed frame. The reason behind this is the fixed frame round trip time, that is typically higher than any normal EDCA or Multi-Poll based approach. The average throughput for first client, when Multi-Poll is being used is approximately $54 \mathrm{Mbps}$ and TDMA with variable timeslot performance is approximately 44 Mbps, depending on the uplink-downlink ratio selection throughput approximately varies between $24 \mathrm{Mbps}$ and $32 \mathrm{Mbps}$. The slow throughput can be partially explained due to slow CPU on the client devices (390 MHz versus $500 \mathrm{MHz}$ where Multi-Poll is being implemented), assumption is that TDMA implementation requires much more processing power, since retransmissions and synchronization is software based unlike the Multi-Poll implementation. However, it is still expected that throughput through 3 client will max out, the TDMA capabilities.
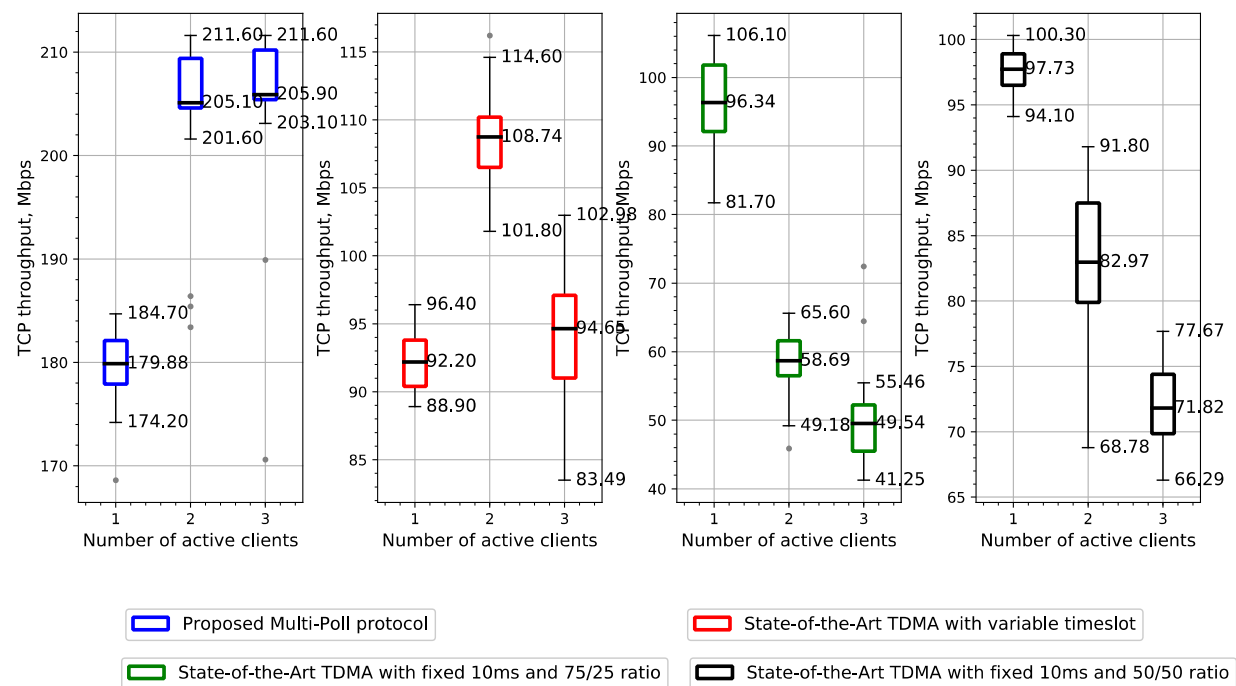

Fig. 3.17. Time Division Multiple Access and Multi-Poll performance with different number of active clients when bidirectional traffic is being sent

The boxplot in Fig. 3.17 represents Multi-Poll and TDMA implementation performance change as number of active client increases. When TDMA system with fixed $10 \mathrm{~ms}$ timeslot has only one active client out of 3 connected, the performance approximately is between $96 \mathrm{Mbps}$ and $98 \mathrm{Mbps}$ depending on the uplink and downlink ratio. The variable timeslot in this case achieves up to 92.2 Mbps. The Multi-Poll in same case achieves approximately $179.88 \mathrm{Mbps}$, that is around $89 \%$ higher compared to the TDMA with variable timeslot, when single active client is present. When all clients are active on TDMA with variable 
timeslot throughput reaches up to $94.68 \mathrm{Mbps}$ and the Multi-Poll achieves 205.9 Mbps on average, while TDMA with $10 \mathrm{~ms}$ timeslot depending on uplink and downlink ratio achieves between $49 \mathrm{Mbps}$ and $71 \mathrm{Mbps}$. Thus, Multi-Poll in comparison with TDMA that uses variable timeslot for data transfer provides up to $117 \%$ higher performance.

\subsection{The Power Allocation in Multi-Poll Access Scheme}

In order to design adaptive transmit power allocation as per GT, an existing IEEE 802.11 $\mathrm{n}$ and ETSI standards (ETSI EN301893 v1.6.1) are being analyzed, which are incorporated into Atheros Software Development Kit (SDK). Adaptive RSSI rate control is based on periodically gathered RSSI. This is done by using Automatic Power Control feature, which provides feedback of statistical information from one wireless device to another. Therefore, both AP and Station knows what signal levels are received on Station or AP side. Such information exchange, allows further usage of Game Theory in wireless system. Furthermore, Automatic Transmit Power Control (ATPC) can be used as an option to adjust power levels. Transmit Power Control (TPC) is defined as a mechanism, which allows to remotely control individual wireless stations transmit power and thus results in decreased interference. TPC can both provide statistical information and adjust power levels on devices or just provide statistical information. Latter is used in control Adaptive RSSI rate control module on stations. RSSI levels is gathered by calculating average of received packets, both control and data packets. It is known that different type of packets can be either sent by lowest modulation as for example beacon or at the highest, in this case MCS15 is used for data packets. As it is known different kind of modulation has a different output power, which can vary between MCS 0 and MCS15 up to 3-4 dBm, depending on the calibration of the individual devices. Furthermore, individual devices due to manufacturing can have a different receiver sensitivity or output power and thus it's a mandatory requirement to evaluate all received packet signal strength while implementing RSSI based rate control. The noise floor is computed by hardware and stored in the noise floor history buffer. The noise floor as defined by Qualcomm is a received power level during clear channel periods and is stored in the registers.

In order to verify the power control algorithm on device, a test scenario was built in outdoor environment consisting of two station devices and one AP, the distance between station devices and AP was approximately $6 \mathrm{~km}$ for first device and $1 \mathrm{~km}$ for second device. During testing it was noticed that when devices are in close proximity with dynamic modulation as well as fallback mechanism enabled they can't achieve target throughput requirements due to noise and due to the 
fact that dynamic modulation decreases data rate and modulation which causes even higher performance decrease between two devices operating on the adjacent frequency. When devices uses lower modulation it takes more time to transmit packet from transmitter to receiver which directly affects the airtime occupation of single device. It can be concluded that in order to use proper power allocation for the devices they have to operate at highest possible modulation without decreasing it unless otherwise it's necessarily. Therefore, proper rate control module operation and WM access scheme is needed to use the possibility of power allocation protocol. Additionally, the Game Theory based Power allocation can be simplified by evaluating the noise floor.

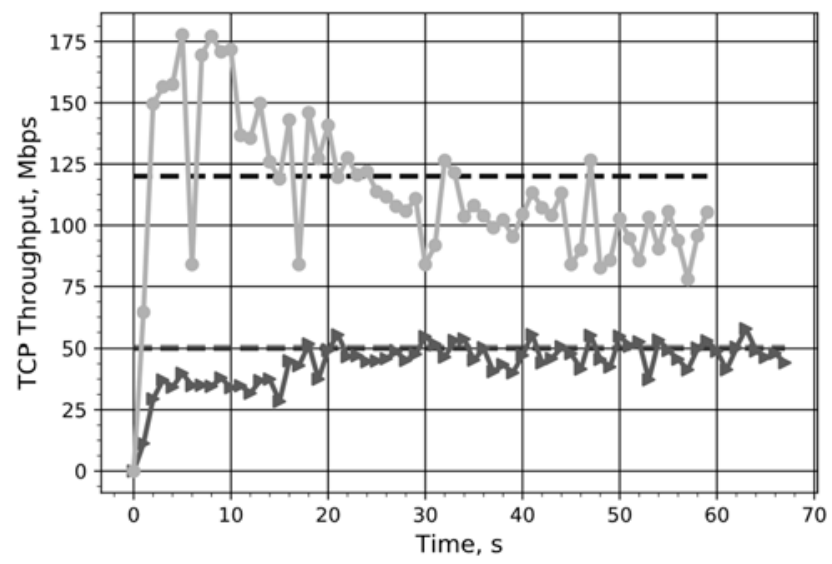

$$
\begin{aligned}
& - \text { - Device1 Target Throughput } \quad-\text { Device2 Target Throughput } \\
& \rightarrow \text { Device1 Achieved Throughput } \quad-\text { Device2 Achieved Throughput }
\end{aligned}
$$

Fig. 3.18. Device 1 and Device 2 target throughput over iterations graph

Finally, it's worth to note, that noise level is typically seen in the co-located towers, where multiple APs are operating in adjacent channel conditions and aren't typically common between station devices in the outdoor based installations. Stations are usually distributed in wider area and due to this power adjustment and control based on the noise level measurement are more effective on the AP side only. Additionally, without the power adjustments, device can adjust receiver's sensitivity level, while setting the transmit power of the connected stations so the received signal level would be higher than the sensed noise level. Furthermore, the devices operating on co-channel conditions should not be limited by the transmit power, since the WM access scheme can handle the airtime sharing, thus using high gain directional antennas would improve both coverage and propagation characteristics (Więcek, Wypiór 2013). The main aim was to evaluate 
if the adjustment of the transmit power can allow to reach the set throughput requirements. The code used to evaluate this was written in Lua language and was verified on the off-the-shelf devices. After disabled the dynamic fallback and set the dynamic rate modulation option to fixed. The test was continued when data rate was set to static fallback as it was noticed if RSSI goes lower than $-66 \mathrm{dBm}$ the device retransmission of packets increases which leads to total throughput decrease almost to 0 . Both devices in test operates on the adjacent frequency according to target throughput requirements. As it can be seen from the Fig. 3.18, the target throughput was met at both devices, however some fluctuations can be seen due to constantly changing environment, since the tests were performed in outdoor environment, where third party devices also compete for the wireless medium.

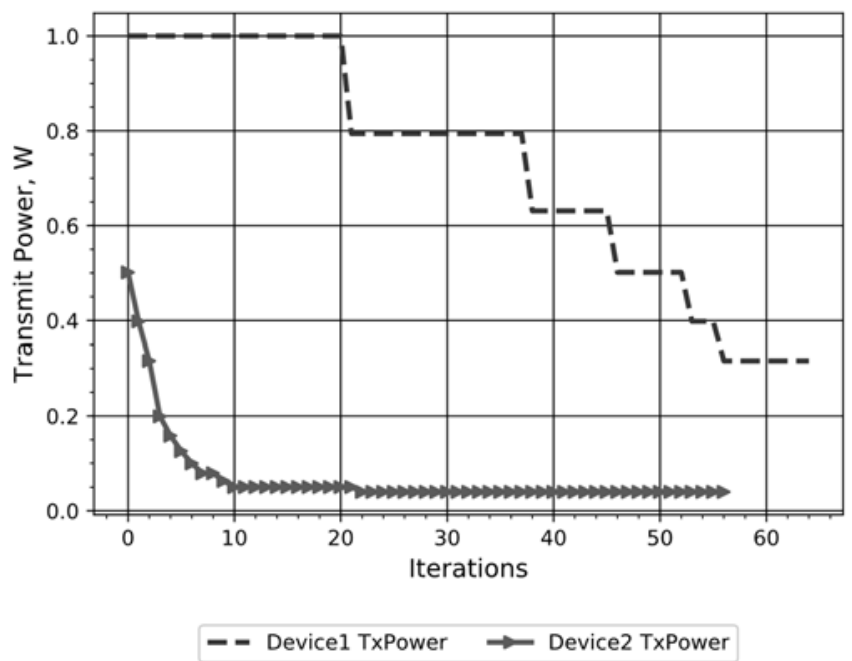

Fig. 3.19. Device 1 and Device 2 Power Allocation Dynamics

The power on Device1 was set at maximum, which allowed to reach throughput of $180 \mathrm{Mbps}$. Since the target throughput was set to $120 \mathrm{Mbps}$, the Device 1 adjusted its power according to throughput requirements by this it let the Device 2 achieve its target throughput while decreasing the total airtime occupation, thus creating fairness between these two devices. While the target throughputs were achieved there still can be seen some fluctuations, since the devicel distance between receiver and transmitter is around $6 \mathrm{~km}$ compared to $1 \mathrm{~km}$ for Device 2. It is worth to note that during all tests there were operating legacy devices nearby on the same frequency, therefore third party devices created some fluctuations during each iteration, however algorithm tried to adjust its target power according to required target throughput requirement for both devices in Fig. 3.19. 
In order to further adapt the power allocation additional information exchange is required, this can be accomplished by using Constrained Application Protocol (CoAP) (Shelby et al. 2014). CoAP is a specialized web transfer protocol with low overhead, which is needed to decrease the amount of traffic generated by wireless nodes. In exchange any statistical information in user space level can be acquired without modifying IEEE 802.11 MAC.

\subsection{Co-Channel Measurements in Outdoor Scenario}

The following measurements were performed in order to evaluate the power allocation impact when there are 2 nearby operating APs in the co-channel, overlapping channel and adjacent channel scenarios. Each of the enabled APs has one connected client. Both APs and clients that are connected to each AP are in close proximity, therefore it is possible to observe power allocation and wireless medium mechanisms operation. It's worth to note, that both of these APs are using Multi-Poll based wireless medium access scheme. As can be seen from Table 3.6 the APs operating in co-channel even with the maximum transmit power allowed by hardware are able to cooperate and share the wireless medium accordingly. However, different case happens when channels are either overlapping or adjacent. Higher transmit power causes higher interference to close proximity devices and thus decreases the overall system capacity. The proper power allocation is a requirement to achieve efficient wireless medium usage. The obtain result is due to that devices are in close proximity and uses sector antennas, therefore wireless medium mechanism isn't effective in such case.

Table 3.6. The performance of two APs operating under different channel allocations

\begin{tabular}{|c|c|c|c|c|c|c|}
\hline $\begin{array}{c}\text { Channel } \\
\text { position }\end{array}$ & $\begin{array}{c}\text { AP } \\
\text { Number }\end{array}$ & $\begin{array}{c}\text { Frequency, } \\
\text { MHz }\end{array}$ & $\begin{array}{c}\text { AP1 Trans- } \\
\text { mit Power, } \\
\mathrm{dBm}\end{array}$ & $\begin{array}{c}\text { AP2 } \\
\text { Transmit } \\
\text { Power, } \\
\mathrm{dBm}\end{array}$ & $\begin{array}{c}\text { AP1, } \\
\text { Mbps }\end{array}$ & $\begin{array}{c}\text { AP2, } \\
\text { Mbps }\end{array}$ \\
\hline Co-channel & $\mathrm{AP} 1$ & 5490 & 29 & 29 & 75 & 82 \\
\cline { 2 - 7 } & $\mathrm{AP} 2$ & 5490 & 12 & 13 & 50 & 53 \\
\hline Overlapping & $\mathrm{AP} 1$ & 5490 & 29 & 29 & 64 & 105 \\
\cline { 2 - 8 } & $\mathrm{AP} 2$ & 5470 & 12 & 13 & 44 & 79 \\
\hline \multirow{2}{*}{ Adjacent } & $\mathrm{AP} 1$ & 5490 & 29 & 29 & 83 & 142 \\
\cline { 2 - 8 } & $\mathrm{AP} 2$ & 5470 & 12 & 13 & 84 & 156 \\
\hline
\end{tabular}

A proper power allocation between devices operating on co-channel allows to increase overall capacity up to $52 \%$ and up to $7 \%$ on adjacent channel if correct 
power allocation is set. The results are reflected in Table 3.6. Therefore, by using smart power allocation mechanism, it is possible to increase both network propagation characteristics as well as the overall system capacity.

\subsection{Multi-band System Experimental Performance Measurements}

Initial performance measurements of $60 \mathrm{GHz}$ with $5 \mathrm{GHz}$ system has been made. The system testing has been performed in indoor environment, which consisted of 4 stations being deployed at various distances, displayed in Fig. 3.20. The Fig. 3.21 displays the total system capacity in Mbps when system operates under different scenarios. In first case single client throughput was measured over PtMP of $60 \mathrm{GHz}$ device. The average aggregated throughput reached up to $1744 \mathrm{Mbps}$, worth to note that Ethernet port is limited by 1000 BaseT. In second run the aggregated throughput over several iterations was measured when all 4 connected clients have been active. The average aggregated throughput resulted in $1501 \mathrm{Mbps}$. It's worth to notice, that one of the clients connected to the $60 \mathrm{GHz}$ band had low signal levels between $-63 \mathrm{dBm}$ and $-68 \mathrm{dBm}$ due to the placement of the device.

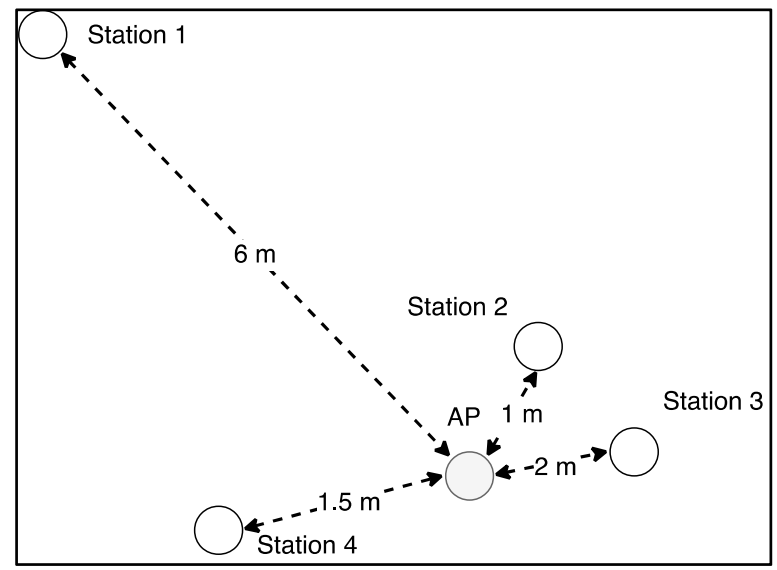

Fig. 3.20. $60 \mathrm{GHz}$ test setup

Finally, the test was conducted by experimentally performing fast session transfer functionality, which is described in IEEE 802.11ad standard, however the standard defines FST operation only when signal is completely lost between $60 \mathrm{GHz}$ client and the PCP. However, in this case the client has been forcefully 
switched to $5 \mathrm{GHz}$ based on the fact that it could not sustain the needed modulation and therefore, the overall system capacity was able to use two bands for data transfer. The measurement for such case was done by running TCP streams over all connected clients and average throughput is approximately up to $1839 \mathrm{Mbps}$. The Fig. 3.22 displays the system performance when active client steering is being used. Thus, the last client in this case was moved to $5 \mathrm{GHz}$ band. After that TCP throughput was generated to verify the system's performance.

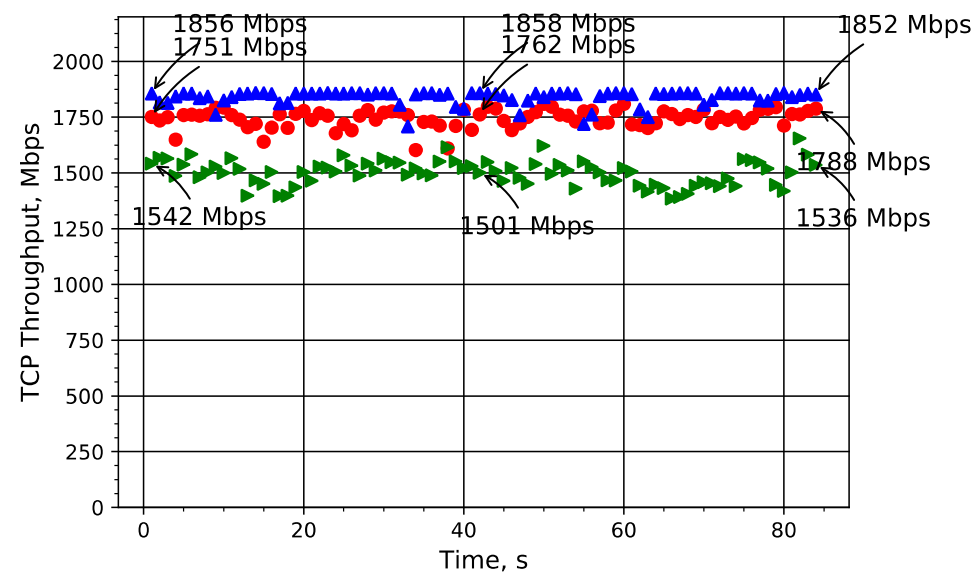

PtP $60 \mathrm{GHz}$ system's capacity

- PtMP $60 \mathrm{GHz}$ with one low data rate client

A PtMP $60 \mathrm{GHz}$ system's capacity when low data rate client is steered using active 5 GHz steering capability

Fig. 3.21. IEEE 802.11 ad performance with Multi-band steering capability
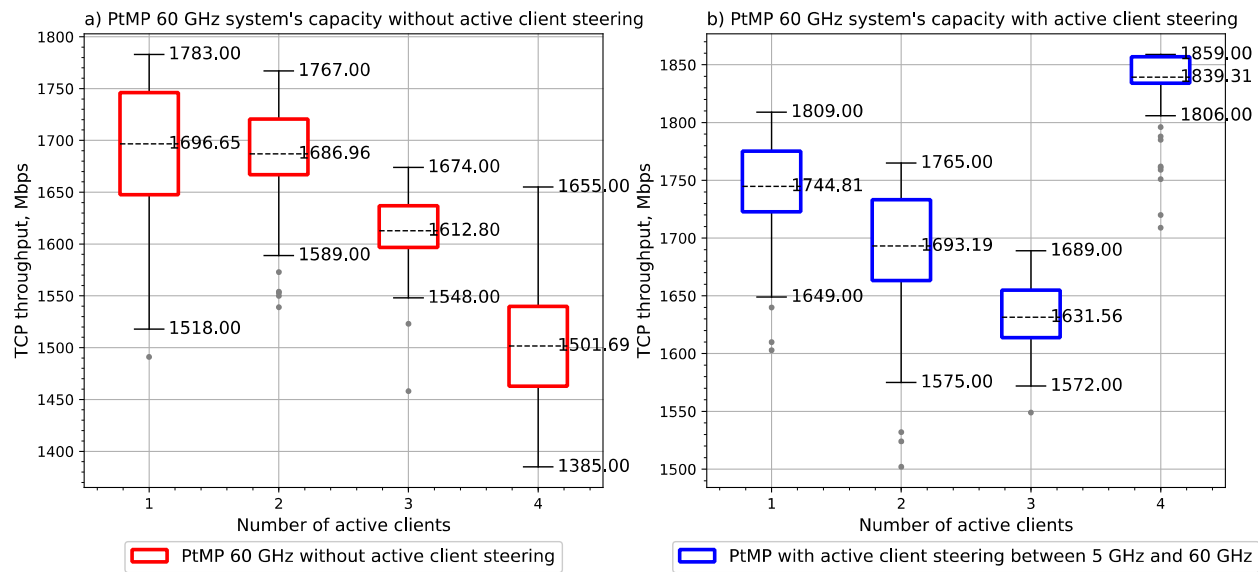

Fig. 3.22. IEEE 802.11 ad performance with Multi-band steering capability when number of active clients is being changed 
As can be seen, the overall system performance increased in comparison when only $60 \mathrm{GHz}$ system was used. In following case, with one active client $60 \mathrm{GHz}$ system without active steering reaches approximately $1696 \mathrm{Mbps}$, while with active steering the performance reaches up to $1744 \mathrm{Mbps}$. Thus, this gives a approximately $3 \%$ throughput increase.

The significant difference appears when the steered client (client 4) is using $5 \mathrm{GHz}$ band instead of $60 \mathrm{GHz}$ band. Due to low signal levels the average performance of the $60 \mathrm{GHz}$ system without active steering reaches up to $1501 \mathrm{Mbps}$, while with active steering $1839 \mathrm{Mbps}$. Therefore, after steering low signal quality clients to $5 \mathrm{GHz}$ the overall system capacity can be increased up to $18 \%$.

\subsection{Conclusions of Chapter 3}

1. A high density scenario when AP served 60 active clients Multi-Poll peaked at $200 \mathrm{Mbps}$ aggregated TCP capacity compared to EDCA $41 \mathrm{Mbps}$ and $133 \mathrm{Mbps}$ for PCF. This allows to conclude, that IEEE 802.11 EDCA with increasing number of active clients isn't able to cope with the number of collisions and thus rate control algorithm decreases the modulation, that results in total system capacity decrease. The same network topology in PCF (without CP) shows higher results and more steady capacity, the same results are seen for Multi-Poll based implementation.

2. Hidden wireless node topology, considers stations located at $5 \mathrm{~km}$ and 200 meters in this case, all these stations are active and generates random number of traffic (using Nepim packet generator). The aggregated downlink throughput for AP is up to $108 \mathrm{Mbps}$ for EDCA, up to $115 \mathrm{Mbps}$ for PCF (without CP) and up to $176 \mathrm{Mbps}$ for MultiPoll in this case.

3. TDMA comparison test was done in indoor environment, in close proximity with 3 active clients. The TDMA setup showed average aggregated TCP capacity of $94 \mathrm{Mbps}$, when using variable frame size, Multi-Poll serving 3 active clients, showed 205 Mbps throughput for the same scenario. This is the factor, that TDMA limits the actual transmission time for the client and thus client is restricted to the boundaries, unlike the Multi-Poll case.

4. In order to build a fully functional multi-band system a proper power allocation mechanism is needed, thus a prototype implementation was compiled on the actual off-the-shelf devices. As can be stated from the measurement results, both of the links operating on the 
same channel were able to reach predefined throughput requirements. Also, it was noticed, that devices operating in close proximity with high gain sectoral antennas requires more accurate power allocation, since near proximity of devices causes an interference and thus degradation of performance can be noticed. Total system capacity can be increased up to $52 \%$ in co-channel operation and up to $7 \%$ in adjacent channel scenario.

5. FST is one of the main benefits of Multi-band system, however IEEE 802.11 ad defines it as a last attempt to move between the bands when signal is being lost of the primary radio. In this case a multi-band considers $5 \mathrm{GHz}$ and $60 \mathrm{GHz}$ as the operational radios. $60 \mathrm{GHz}$ being the primary radio, while $5 \mathrm{GHz}$ acts as a backup.

6. A new approach has been taken for FST, which includes active client steering technique, which is not defined or analyzed in literature. The active client steering technique is an approach that allows to move less active clients (Multi-Poll similarity) to secondary band, in this case $5 \mathrm{GHz}$ and more active clients, which require specific throughput requirements to $60 \mathrm{GHz}$ band. Additionally, such steering allows in advance to move clients, which underperform (have low modulations on $60 \mathrm{GHz}$ band). By using such attempt, it has been experimentally verified, that such multi-band system, can provide up to $18 \%$ higher system throughput in $60 \mathrm{GHz}$ point to multipoint. 



\section{General Conclusions}

1. Evaluation of IEEE 802.11n Media Access Control and practical experiments concludes that IEEE 802.11 efficiency is highly dependent on the density of wireless devices. Moreover, it was concluded that IEEE 802.11 EDCA can operate only with up to 10 of active clients. When number of active clients increases above the mentioned number, the system performance starts decreasing.

2. Theoretical analysis of Media Access Control layer identified that NAV parameter is not used properly and therefore co-location with TDMA based devices in the same co-channel results in high packet loss.

3. From the evaluation of MAC IEEE 802.11 and practical experiments it can be concluded, when a high number of clients is connected to $\mathrm{AP}$, which is a typical scenario in point-to-multipoint networks, the existing IEEE 802.11 EDCA mechanism cannot cope with handling wireless medium. However, the Multi-poll based solution can increase throughput since stations are moved between active and idle state. Thus, it can be concluded that Multi-Poll is efficient in point to multipoint outdoor based scenarios to provide last mile communications services. 
4. With 13 active clients Multi-Poll provides up to $79 \%$ higher data throughput in comparison with EDCA and up to $12 \%$ higher data throughput in comparison PCF (without CP), when TCP traffic is being sent. When system contains 60 active clients, Multi-Poll protocol reaches up to $50 \%$ higher system capacity in comparison with PCF (without CP) and up to $388 \%$ higher system capacity in comparison with EDCA.

5. Theoretical and practical experiments showed that Game Theory can increase overall system capacity when proper power settings are performed based on the environmental factors, overall system capacity, when two APs are operating on adjacent channels with correct power allocation can be increased approximately $7 \%$ and up to $52 \%$ operating on co-channel. Such incorporation together with Multi-Poll would allow even more efficient channel usage for data transmissions.

6. The FST approach which allows to combine several IEEE 802.11 based technologies operating on different frequencies allowed to increase QoE for end customer, this is achieved by leveraging the different activity and idling periods between clients. The practical experiment and conceptual model shows, that such performance increase due to leveraging technologies is in line with $5 \mathrm{G}$ scope, which is currently being decided by State-of-the-art LTE vendors like Qualcomm or Huawei. Additionally, the performance increase due to leveraging such technologies allows to achieve up to $18 \%$ higher data throughput.

7. It has been confirmed that Multi-Poll is able to operate both in cochannel conditions as well as in adjacent conditions with proper power allocation scheme even with legacy EDCA based devices. 


\section{References}

Abe, A.; Walker, D. 2016. Multi-hop 802.11 ad wireless H.264 video streaming, Telecommunications and Signal Processing (TSP), 2016 39th International Conference on, 2016, 94-99.

Afaqui, S.; Villegas E.; López-Aguilera E. 2017. IEEE 802.11ax: Challenges and requirements for future high efficiency WiFi, IEEE Wireless Communications, 24(3): 130-137.

Assasa, H.; Loch, A.; Widmer, J. 2016. Packet mass transit: Improving frame aggregation in $60 \mathrm{GHz}$ networks, World of Wireless, Mobile and Multimedia Networks (WoWMoM), 2016 IEEE 17th International Symposium on A, 2016, 1-7.

Baid, A.; Raychaudhuri, D; 2015. Understanding channel selection dynamics in dense WiFi networks, IEEE Communications Magazine 53(1): 110-117.

Bankov, D.; Didenko, A.; Khorov, E.; Loginov, V.; Lyakhov, A. 2017. IEEE 802.11ax Uplink Scheduler to Minimize Delay: a Classic Problem with New Constraints, IEEE 28th Annual International Symposium on Personal, Indoor, and Mobile Radio Communications (PIMRC), 2017, 1-5.

Bellalta, B; Kosek-Szott, K. 2017. AP-initiated Multi-User Transmissions in IEEE 802.11ax WLANs, arXiv preprint arXiv:1702.05397, 2017, 1-10.

Belmega, V.; Djeumou, B.; Lasaulce, S. 2010. Power allocation games in interference relay channels: Existence analysis of nash equilibria. EURASIP Journal on Wireless Communications and Networking, 2010, 1-21. 
Bianchi, G. 2000. Performance Analysis of the IEEE 802.11 Distributed Coordination Function, IEEE Journal on Selected Areas in Communications 18(3): 535-547.

Brouer, D.; Hansen, S. 2004. Experiences with Reducing TCP Performance Problems on ADSL, DIKU - Technical Report 04/07, 2004, 1-6.

Broustis, I.; Eriksson, J.; Krishnamurthy, V.; Faloutsos, M. 2007. Implications of Power Control in Wireless Networks: A Quantitative Study, In Passive and Active Measurement Conference (PAM), 2007, 88-93.

Bruno, R.; Conti, M.; Gregori, E. 2002. IEEE 802.11 Optimal Performances: RTS/CTS mechanism vs Basic Access, Proceeding of the $13^{\text {th }}$ PIMRC 2002, 1747-1751.

Bruzgiene, R.; Narbutaitè, L.; Adomkus, T.; Cibulskis, R. 2014. The Impact of Video Streaming on Quality of Experience over 802.11n Dual Band networks: Experimental Evaluation. Elektronika ir Elektrotechnika 20(6): 158-161.

CEPT ECC Report 200. 2013. Co-existence studies for proposed SRD and RFID applications in the frequency band 870-876 MHz and 915-921 MHz. 198 p. Available online from: http://www.erodocdb.dk/

Chandra, K.; Cao, Z.; Bruintjes, M.; Prasad, R.; Karagiannis, G.; Tangdiongga, E.; Boom, A.; Kokkeler, J. 2015. mCRAN: A radio access network architecture for 5G indoor communications, Communication Workshop (ICCW), 2015 IEEE International Conference on, 2015, 300-305.

Chandra, K.; Prasad, V.; Niemegeers I. 2017. Performance Analysis of IEEE 802.11ad MAC Protocol, IEEE Communications Letters 21(7): 1513-1516.

Chandra R.; Mahajan, R.; Moscibroda, T.; Raghavendra, R.; Bahl P. 2008. A case for adapting channel width in wireless networks. In ACM SIGCOMM computer communication review 38(4): 135-146.

Chatzimisios, P.; Boucouvalas, A.; Vitsas, V. 2004. Effectiveness of RTS/CTS handshake in 802.11a Wireless LANs, IEEE 2004 Electronics Letters Online 40(14): 915-916.

Chaudet, C.; Haddad Y. 2013. Wireless Software Defined Networks: Challenges and opportunities, IEEE International Conference on Microwaves, Communications, Antennas and Electronics Systems (COMCAS), 2013. 1-5.

Chávez-Santiago, R.; Haddad, Y.; Lyandres, V.; Balasingham, I. 2015. VoIP Transmission in Wi-Fi Networks with Partially-Overlapped Channels, in Proc. of IEEE Wireless Communications and Networking Conference (WCNC), March 2015, New Orleans, USA, 2015, 340-345.

Chen, J.; Lin, C.-A; 2004. HMM: Hybrid Multipolling Mechanism with Pre-allocation Admission Control for Real-Time Transmissions in WLANs, in IEEE 60th Vehicular Technology Conf. (VTC2004-Fall), 2004, 3040-3044.

Chen, C.; Sivalingam K.M.; Agrawal P.; Kishore S. 1998. A comparison of MAC protocols for wireless local networks based on battery power consumption, INFOCOM '98. Seventeenth Annual Joint Conference of the IEEE Computer and Communications Societies. Proceedings, 1998, 150-157. 
Chen, Q.; Tang, J.; Wong, C.; Peng, X.; Zhang, Y. 2013. Directional Cooperative MAC Protocol Design and Performance Analysis for IEEE 802.11ad WLANs, IEEE Transactions on Vehicular Technology 62( 6): 2667-2677.

Chen, Z.; Khokhar, A; 2003. Improved MAC Protocols for DCF and PCF Modes over Fading Channels in Wireless LANs, in 2003 IEEE Wireless Commun. and Networking, WCNC 2003, New Orleans, LA, USA, 2003, 1297-1302.

Cheng, S.; Wu, M. 2009. Contention-Polling Duality Coordination Function for IEEE 802.11 WLAN Family, IEEE Trans. on Commun., 57(3): 779-888.

Choi, W.; Kim, H.; Kim, Il-Gyu. 2012. A system design for IEEE802.11ad. Communications (APCC), 2012 18th Asia-Pacific Conference on, 2012, 2012, 668-671.

Choi, S.; Prado, J.; Sai, N.; Mangold, S. 2003. IEEE 802.11e Contention-Based Channel Access (EDCF) Performance Evaluation, Communications, 2003. ICC '03. IEEE International Conference on 2: 1151-1156.

Chou, H.; Lin, Woei. 2013. Performance Analysis of Packet Aggregation for IEEE 802.11 PCF MAC-Based Wireless Networks, Wireless Communications, IEEE Transactions on 12(4):1441-1447.

Clark D. D. 1982. Window and Acknowledgment strategy in TCP. RFC813, 1982. 22 p. [Online]. Available: https://tools.ietf.org/html/rfc813

Cordeiro, C. 2013. The Pursuit of Tens of Gigabits per Second Wireless Systems, IEEE Wireless Communications, 2013, 3-5.

Dasari, M. 2017. Real time detection of MAC layer DoS attacks in IEEE 802.11 wireless networks, Consumer Communications \& Networking Conference (CCNC), 2017 14th IEEE Annual, 2017, 939-944.

Deek. L.; Garcia-Villegas E.; Belding, E.; Lee, SJ.; Almeroth, K. 2011. The Impact of Channel Bonding on 802.11n Network Management, CoNEXT'11 Proceedings of the Seventh Conference on emerging Networking Experiments and Technologies 11, 2011, 1-12.

Dekeris, B.; Narbutaitè, L. 2007. The Measurement of WLAN Throughput Using Different Security Protocols, Proceedings of international conference on Communications, Electromagnetics and Medical Applications (CEMA'07), Sofia, Bulgaria, 25th-27th October, 2007 / Technical University of Sofia, Bulgaria; National Technical University of Athens, Greece. Sofia : Technical University of Sofia, 2007, 1-5.

Dimatteo, S.; Hui, P.; Han, B.; Li, V. O. K. 2011. Cellular traffic offloading through WiFi networks, in Proc. of the IEEE 8th International Conference on Mobile Ad-Hoc and Sensor Systems (MASS), Valencia, Spain, October 17-22, 2011, 192-201.

Diwakar, K.; Iyer, S; 2004. Supporting Real-Time Applications with Better QoS Guarantees in 802.11, Wireless Communication Systems, 2004, 1st International Symposium on, 2004, 1-5.

Doering, M.; Budzisz, L.; Wilkomm, D.; Wolisz. 2013. About the practicality of using partially overlapping channels in IEEEE $802.11 \mathrm{~b} / \mathrm{g}$ networks, Proc. of IEEE International Conference on Communications (ICC), 2013, 3703-3707. 
Dua, A.; Kumar, N.; Bawa, S.; Chilamkurti, N. 2013. Efficient TDMA based Virtual Back off Algorithm for Adaptive Data Dissemination in VANETs, Wireless and Pervasive Computing (ISWPC), 2013 International Symposium on, 2013, 1-6.

Etkin, R.; Parekh, A.; Tse, D. 2007. Spectrum sharing for unlicensed bands, IEEE J. Select. Areas Comm. 25: 517-528.

ETSI TS 136101 V10.3.0 (2011-06) Technical Specification. 2011. Evolved Universal Terrestrial Radio Access (E-UTRA); User Equipment (UE)radio transmissin and Reception(3GPP TS 36.101 V 10.3.0 Release 10. Section 7.5 Adjacent Channel Selectivity, 236 p.

Fallah, Y. P.; Alnuweiri, H. 2007. Hybrid polling and contention access scheduling in IEEE 802.11e WLANs, J. of Parallel and Distributed Computing 67(2): 242-256.

Fang, G.; Dutkiewicz, E.; Yu, K.; Vesilo, R., Yu Y. 2010. Distributed Inter-Network Interference Coordination for WBANs, Proc. IEEE Globecom 2010, 1-5.

Federal Communications Commission 1985. Authorization of Spread Spectrum Systems Under Parts 15 and 90 of the FCC Rules and Regulations. 16 p.

Felegyhazi, M.; Hubaux, J. P. Game Theory in Wireless Networks: A Tutorial, EPFL Technical report: LCA-REPORT-2006-002, Switzerland, Jan. 2006, 1-15.

Feng, W.; Li, Y.; Jin, Depeng; Zeng, L. 2014. Inter-network spatial sharing with interference mitigation based on IEEE 802.11ad WLAN system, Globecom Workshops (GC Wkshps), 2014, 752-758.

Feng, W.; Niu, Y.; Li, Y.; Gao, Bo; Su, Li; Jin, D.; Wu, D. O. 2016. Throughput enhancement of IEEE 802.11ad through space-time division multiple access scheduling of multiple co-channel networks, IET Communications 10(4): 425-434.

Fiehe, S.; Riihijarvi, J.; Mahonen, P. 2010. Experimental Study on Performance of IEEE $802.11 \mathrm{n}$ and Impact of Interferers on the $2.4 \mathrm{GHz}$ ISM Band, IWCMC '10, 2010, 47-51.

Gast, Matthew. 802.11ac: A Survival Guide. O'Reilly Media, 2013. 100 p.

Guillory, J.; Tanguy, E.; Pizzinat, A.; Charbonnier, B.; Meyer, S.; Algani, C.; Li, H. 2011. A $60 \mathrm{GHz}$ Wireless Home Area Network With Radio Over Fiber Repeaters, Journal of Lightwave Technology 29(16): 2482-2488.

Guo, F.; Chiueh, T. 2007. Software TDMA for VoIP Applications over IEEE802.11 Wireless LAN, INFOCOM 26th IEEE International Conference on Computer Communications, 2007, 2366-2370.

Haghani, E.; Krishnan, M.; Zakhor, A. 2010. Adaptive Carrier Sensing for Throughput Improvement in IEEE 802.11 Networks, Proceedings of the IEEE Globecom, 2010, 1-6.

Hanna, S.; Sydor, J. 2012. Distributed sensing of Spectrum Occupancy and Interference in Outdoor 2.4 GHz Wi-Fi networks, Proceedings of the Global Communications Conference 2012: 1453-145.

Hardin, Garrett. 2007. The tragedy of the unmanaged common, Evolutionary perspectives on environmental problems, 2007, 105-107. 
Hemanth, C.; Venkatesh, T. G. 2014. Performance analysis of contention-based access periods and service periods of 802.11 ad hybrid medium access control, IET Networks 3(3): 193-203.

Hemanth, C.; Venkatesh T. G. 2016. Performance Analysis of Service Periods (SP) of the IEEE 802.11ad Hybrid MAC Protocol, IEEE Transactions on Mobile Computing 15, (5): 1224-1236.

Heusse, M.; Rousseau, F.; Berger-Sabbatel G.; Duda, A. 2003. Performance anomaly of 802.11 b, Proceedings of the IEEE International Conference on Computer Communication (INFOCOM), April 2003, 2003, 836-843.

Hills, A. 2001. Large Scale Wireless LAN Design, IEEE Communications Magazine November 2001, 39(11) 98-107.

Høiland-Jørgensen, T. 2016. Flent: The FLExible Network Tester [Online]. Available: https://flent.org/flent-the-flexible-network-tester.pdf

Hsieh, Tsung-Han; Lin, Y.; Wang, P.C. 2015. A Hybrid MAC Protocol for Wireless Sensor Network, Networking, Sensing and Control (ICNSC), 2015 IEEE 12th International Conference on, 2015, 93-98.

Fang, Y.; Gu, D.; McDonald A. B.; Zhang J. 2005. On the Performance Enhancement of Wireless LAN - A Multi-polling Mechanism with Hidden Terminal Solution, in 2005 Global Telecommun. Conf. (GLOBECOM'05), 2005, 571-575.

Yamada, T.; Nishio, T.; Morikura, M.; Yamamoto, K. 2015. Experimental evaluation of IEEE 802.11ad millimeter-wave WLAN devices, Communications (APCC), 201521 st Asia-Pacific Conference on, 2015, 278-282.

Yang, Z.; Zhang, J.; Tan, K.; Zhang, Q.; Zhang, Y. 2015. Enabling TDMA for Today's Wireless LANs, Computer Communications (INFOCOM), 2015 IEEE Conference on, 2015, 1436-1444.

IEEE 802.11 WG. 2012. IEEE 802.11ad, Amendment 3:Enhancements for Very High Throughput in the $60 \mathrm{GHz}$ Band, 2012, $628 \mathrm{p}$.

IEEE Std 802.11-2016. 2016. (Revision of IEEE Std 802.11-2012) - IEEE Standard for Information technology-Telecommunications and information exchange between systems Local and metropolitan area networks-Specific requirements - Part 11: Wireless LAN Medium Access Control (MAC) and Physical Layer (PHY) Specifications, 2016, $3534 \mathrm{p}$.

Institute of Electrical and Electronic Engineering (IEEE) Standard: Part 11, Wireless LAN Medium Access Control (MAC) and Physical Layer (PHY) Specifications: High-Speed Physical Layer in the $5 \mathrm{GHz}$ Band IEEE 802.11a. 1999, 96 p.

Jain, R.; Chiu, D.; Hawe, W. 1984. A Quantitative Measure of Fairness and Discrimination for Resource Allocation in Shared Systems, Digital Equipment Corporation, Technical Report DEC-TR-301, Tech. Rep., 1984, 38 p.

Japertas, S.; Slanys, R. 2013. Research of IEEE 802.11 Standard Signal Propagation Features in Multi Floor Buildings. Electronics and Electrical Engineering 19(8): 101- 104. 
Jardosh, A.; Ramachandran, K.; Almeroth, K; Belding-Royer, E. 2005. Understanding Congestion in IEEE 802.11b Wireless Networks, Proceedings of the 5th USENIX conference on Internet Measurement IMC, 2005, 1-14.

Jiang, S.; Peng, J.; Lu, Zhi, Jiao, J.; Jiang, S. 2014. 802.11ad Key Performance Analysis and Its Application in Home Wireless Entertainment, Computational Science and Engineering (CSE), 2014 IEEE 17th International Conference on, 2014, 1595-1598.

Jun, F.; Zhitang, Li; Li, W.; Yuan, C. 2006. SCSR: A Novel MAC Protocol for Mobile Ad Hoc Network, Wireless Communications, Networking and Mobile Computing, 2006, $1-4$.

Kalfas, G.; Pleros, N.; Alonso, L.; Verikoukis, C. 2016. Network planning for 802.11ad and MT-MAC $60 \mathrm{GHz}$ fiber-wireless gigabit wireless local area networks over passive optical networks, IEEE/OSA Journal of Optical Communications and Networking 8(4): 206-220.

Kazemi, R.; Vesilo, R.; Dutkiewicz, E.; Liu, Ren. 2011. Dynamic power control in wireless body area networks using reinforcement learning with approximation, in Proceedings of Personal Indoor and Mobile Radio Communications (PIMRC) IEEE 22nd International Symposium, Canada, September 2011, 2203-2208.

Kemerlis, V.; Stefanis, E.; Xylomenos, G.; Polyzos, G. 2006. Throughput Unfairness in TCP over WiFi, in proc. of the Third Annual Conference on Wireless On-demand Network Systems and Services 2006, 1-6.

Khan, Y.; Derakhshani, M.; Parsaeefard, S.; Le-Ngoc, Tho. 2015. Self-Organizing TDMA MAC Protocol for Effective Capacity Improvement in IEEE 802.11 WLANs, Globecom Workshops (GC Wkshps), 2015, 1-6.

Kim, B.-S.; Kim, S. W.; Fang, Y; Wong, T. F. 2005. Two-Step Multipolling MAC Protocol for Wireless LANs, IEEE J. on Selected Areas in Commun 23(6): 1276-1286.

Kim, J.; Tian, Y.; Molisch, A. F.; Mangold. S. 2011. Joint optimization of HD video coding rates and unicast flow control for IEEE 802.11 ad relaying. Personal Indoor and Mobile Radio Communications (PIMRC), IEEE 22nd International Symposium on, 2011, 1109-1113.

Kiryanov, A.; Krotov, A.; Gallo, P.; Garlisi, D.; Tinnirello, I. 2016. Joint Usage of Dynamic Sensitivity Control and Time Division Multiple Access in Dense 802.11ax Networks, International Workshop on Multiple Access Communications 2016, Springer 10121: 57-71.

Klein, P. 2008. IEEE 802.11 QoS Overview, IEEE Plenary Meeting IEEE Plenary Meeting - Nov 08 Dallas, TX Dallas, TX 2008, 38 p.

Kosek-Szott, K.; Szott, S.; Natkaniec, M.; Prasnal, L. 2017. Coexistence Issues in Future WiFi Networks. IEEE Network 31(4): 86-95.

Lee, II.-Gu; Kim, M. 2016. Interference-aware self-optimizing Wi-Fi for high efficiency internet of things in dense networks, Computer. Communications, 58: 60-74. 
Lee, S. J.; Kim, K; Chang, K; Kyeong, M. G.; Lee, W.; Chung, H. K. 2011. Evaluation of $60 \mathrm{GHz}$ MIMO Channel Capacity in the Conference Room STA-STA Scenario, Vehicular Technology Conference (VTC Spring), 2011, 1-5.

Leffler, S. Errno Consulting. 2009. TDMA for Long Distance Wireless Networks, University of Cambridge, Computer Laboratory Seminar, 2009, 1-10.

Li, Chen; Cai, Ran; Liu, D. 2014. A suboptimal STDMA scheduling for concurrent transmissions in mmWave wireless networks, Signal Processing, Communications and Computing (ICSPCC) IEEE International Conference on, 2014, 137-141.

Li, Nanxi; Wei, Z.; Geng, J.; Sang, Lin; Yang, D. 2014. Multiuser hybrid beamforming for max-min SINR problem under $60 \mathrm{GHz}$ wireless channel, Personal, Indoor, and Mobile Radio Communication (PIMRC), 2014 IEEE 25th Annual International Symposium on, 2014, 123-128.

Li, W.; Wei, B.; Wang, S. 2007. An Evolutionary-Dynamic TDMA Slot Assignment Protocol for Ad Hoc Networks, Wireless Communications and Networking Conference, 2007.WCNC 2007, 138-142.

Li, W.; Wei, B.; Wang, S. 2006. Dynamic TDMA Slot Assignment Protocol for Multi Hop Adhoc Networks, Communication Technology, 2006, 1-6.

Liaw, H.; Yeh, T. 2011. A TDMA Medium Access Control Mechanism for IEEE 802.11based Wireless Networks, Genetic and Evolutionary Computing (ICGEC), 2011 Fifth International Conference on, 2011, 61-64.

Lin, J.; Wu, C.; Ohzahata, S.; Kato, T. 2014. A QoS Supporting Ad Hoc Network Protocol Combing Admission Based TDMA and 802.11 DCF, Network Operations and Management Symposium (APNOMS), 2014, 1-4.

Liu, J.; Yao, M.; Qiu, Z. 2016. Enhanced BlockACK method for A-MPDU transmission in IEEE 802.11n/ac/ad WLANs, Electronics Letters 52(2): 159-161.

Lomayev, A.; Gagiev, Y.; Ershov, I.; Maltsev, A.; Genossar, M.; Bogdanov, M. 2016. Experimental investigation of $60 \mathrm{GHz}$ WLAN channel for office docking scenario, Antennas and Propagation (EuCAP), 2016 10th European Conference on, 2016, 1-5.

Matusevičius, A.; Adomkus, T.; Narbutaitė, L.; Brūzgienè, R. 2015. IEEE 802.11AC standard impact on transmission of VoIP service, Journal of applied electromagnetism. Athens: National Technical University of Athens: School of Electrical \& Computer Engineering 17(2): $23-38$.

Medeisis, A.; Sydor, J.; Cremene, L.; Holland, O.; Anskaitis, A.; Wiecek, D.; Haddad, Y.; Cuzanauskas T. 2014. ISM-Advanced: Improved Access Rules for Unlicensed Spectrum, IEEE Int. Symp. on Dynamic Spectrum Access Networks (DYSPAN), McLean, VA, 2014, 194-205

Mohamed, M.; Sakaguchi, K.; Sampei, S. 2016. Wi-Fi/WiGig Coordination for Optimal WiGig Concurrent Transmissions in Random Access Scenarios, Vehicular Technology Conference (VTC Spring), 2016, 1-5. 
Munari, A.; Rossetto, F.; Zorzi, M. 2011. Impact of Medium Access Control Strategies on the Effectiveness of Advanced Cooperative Hybrid ARQ Techniques, IEEE Transactions on Wireless Communications 10(9): 2860-2871.

Nachtigall, J.; Zubow, A.; Redlich, J. P. 2008. The Impact of Adjacent Channel Interference in Multi-Radio System using IEEE 802.11, International Wireless Communications and Mobile Computing Conference IWCMC, 2008, 874-881.

Natkaniec, M.; Kowalski, R.; Kostecki, K. 2015. Analiza zjawiska anomalii wydajności w sieciach standardu IEEE 802.11ac, Przegląd Telekomunikacyjny, Wiadomości Telekomunikacyjne 88(4): 256-259.

Nikolikj, V.; Janevski, T. 2014. Profitability and comparative cost-capacity analysis of 5G millimeter-wave systems, Telecommunications Forum Telfor (TELFOR), 2014, 256-259.

Nitsche, T.; Cordeiro, C.; Flores, B.; Knightly, W.; Perahia, E.; Widmer, C. 2014. IEEE 802.11ad: directional $60 \mathrm{GHz}$ communication for multi-Gigabit-per-second Wi-Fi, IEEE Communications Magazine 52(12): 132-141.

Nupur, K.; Mahajan, R.; Millstein, T.; Govindan, R.; Musuvathi, M. 2011. Finding Protocol Manipulation Attacks, SIGCOMM Computer Communication Review 41: 26-37.

Djukic P.; Mohapatra P. Soft-TDMAC: A Software TDMA-based MAC over Commodity 802.11 hardware. INFOCOM 2009, 1836-1844.

P.1238. 2001. Propagation data and prediction methods for the planning of indoor radio communication systems and the radio local area networks in the frequency range $900 \mathrm{MHz}$ to $100 \mathrm{GHz}$, ITU-R Recommendation P.1238, ITU, Geneva, 2001, 28 p.

Mashinchi-Ghabel P.; Hossein A.; Manzuri-Shalmani T. A solution on deafness problem of WiGig networks based on IEEE 802.11ad. Wireless Days, 2017, 1-3.

Park, H.; Park, S.; Song, T.; Pack, S. 2013. An Incremental Multicast Grouping Scheme for mmWave Networks with Directional Antennas, IEEE Communications Letters 17(3): 616-619.

Parker, C.; Koczian, G.; Quinlan, T.; Walker D. 2015. High capacity communications at $24 \mathrm{GHz}$ and $60 \mathrm{GHz}$ for converged 5G networking, Networks and Optical Communications - (NOC), 2015 20th European Conference on, 2015, 1-6.

Patras, P.; Banchs, A.; Serrano, P. 2012. A control theoretic scheme for efficient video transmission over IEEE 802.11e EDCA WLANs. ACM Trans. Multimedia Comput. Commun. Appl. 8(3): 1-23.

Pauliukas, D.; Vosylius, V. 2005. Research of Real Time Traffic Transmission in 802.11 WLANs. Elektronika ir Elektrotechnika 7(95): 111-114.

Pavilanskas, L.; Statkus, A. 2010. Evaluation of TCP Acknowledgment Mechanism Influence on Router Performance, Research J. Elektronika ir Elektrotechnika 103(7): 95100 . 
Pavon, J. P.; Choi, S. 2003. Link adaptation strategy for IEEE 802.11 WLAN via received signal strength measurement, Communications, 2003. ICC'03. IEEE International Conference on 2, 2003, 1108-1113.

Pediaditaki, S.; Marina, M. K.; Tyrode, D. 2012. Traffic-aware channel width adaptation in long-distance 802.11 mesh networks, In Proceedings of the 15th ACM international conference on Modeling, analysis and simulation of wireless and mobile systems 2012, 261-270.

Perahia, E.; Stacey, R. 2013. Next Generation Wireless LANs: 802.11n and 802.11ac, Cambridge University Press, New York, NY, 2013, 416 p.

Python language programme used for simulation of power control game. Available online in the document download area at: http://www.cost-terra.org/our-publications .

Qualcomm Wireless LAN Access Point (Driver Version 10.4) Programmer's guide 2018, $1267 \mathrm{p}$.

Qualcomm, "Extending the benefits of LTE Advanced to unlicensed spectrum", November 2013, 19 p.

Rajan, U.; Babu, V. 2016. Saturation throughput analysis of IEEE 802.11ad wireless LAN in the contention based access period(CBAP), Distributed Computing, VLSI, Electrical Circuits and Robotics (DISCOVER), 2016, 41-46.

Raman, B.; Chebrolu, K. Experiences in using WiFi for Rural Internet in India, IEEE Communications Magazine 45(1): 104-110.

Report for the European Commission, 2013. Study on Impact of traffic off-loading and related technological trends on the demand for wireless broadband spectrum, 2013, $168 \mathrm{p}$. Available in the EU Bookshop at: http://dx.doi.org/10.2759/19531

Rindzevičius, V.; Grimaila V.; Pilkauskas V.. 2006. Traffic analysis of the next generation wireless access technologies, Amalfi, Italy, March 23-26, 2006 / IPSI Belgrade, Serbia. Belgrade : IPSI Belgrade Academic Mind, 2006, 1-7.

Ruckus. ChannelFly — Predictive Capacity Management for Smarter RF Channel Selection. Available online at: http://www.ruckuswireless.com/technology/channelfly

Saha, S. K.; Garg, A.; Koutsonik, D. 2015. A first look at TCP performance in indoor IEEE 802.11ad WLANs. Computer Communications Workshops (INFOCOM WKSHPS), 2015 IEEE Conference on, 2015, 63-64.

Saha, S. K.; Vira, V.; Garg, A.; Koutsonikolas, D. 2016. Feasibility Study of $60 \mathrm{GHz}$ Indoor WLANs. Computer Communication and Networks (ICCCN), 2016 25th International Conference on, 2016, 1-9.

Sharon, O.; Alpertb, Y. 2016. Coupled IEEE 802.11ac and TCP performance evaluation in various aggregation schemes and Access Categories, Computer Networks 100: 141156.

Shelby, Z.; Hartke, K.; Bormann, C.; Frank, B. RFC 7252. 2014. Constrained Application Protocol (CoAP), 2014, 112 p. Available online: http://tools.ietf.org/html/rfc 7252 
Shen, Q.; Fang, X.; Huang, R.; Li, P.; Fang, Y. 2009. Improving Throughput by tuning carrier sensing in 802.11 wireless networks, Journal Computer Communications 32(11): 1263-1270.

Shi, C.; Dai, X.; Zhou, Qi; Nie, J. 2010. A Novel Fixed Contention Window Backoff Algorithm for IEEE 802.11 WLAN, Multimedia Information Networking and Security (MINES), 2010 International Conference on, 2010, 174-177.

Shoaei, D.; Derakhshani, M.; Parsaeefard, S.; Le-Ngoc, Tho. 2015. Learning-based Hybrid TDMA-CSMA MAC Protocol for Virtualized 802.11 WLANs, Personal, Indoor, and Mobile Radio Communications (PIMRC), IEEE 26th Annual International Symposium on, 2015, 1861-1866.

Shrivastava, V.; Rayanchu, S.; Yoon, J.; Banerjee, S. 2008. 802.11n Under the Microscope, IMC 2008 Proceedings of the 8th ACM SIGCOMM conference on Internet Measurement, 2008, 105-110.

Singh, J.; Li, Yan; Bambos N.; Bahai, A.; Bangnan, Xu.; Zimmermann, G. 2007. TCP Performance Dynamics and Link-Layer Adaptation Based Optimization Methods for Wireless Networks. Wireless Communications, IEEE Transactions on. 6. 1864-1879.

Singh, S.; Ziliotto, F.; Madhow, U.; Belding, E.; Rodwell M. 2009. Blockage and directivity in $60 \mathrm{GHz}$ wireless personal area networks: From cross-layer model to multihop MAC design. Selected Areas in Communications, IEEE Journal on. 27: 1400-1413.

Srinivasan, K.; Levis, P. 2006. RSSI is Under Appreciated, In Proceedings of the Third Workshop on Embedded Networked Sensors (EmNets 2006), 2006, 1-5.

Statkus, A.; Paulikas, S. 2013. Improving TCP Performance in IEEE 802.11 Networks, Research J. Elektronika ir Elektrotechnika 19(5): 99-102.

Sum, S.; Harada, H. 2012. Scalable Heuristic STDMA Scheduling Scheme for Practical Multi-Gbps Millimeter-Wave WPAN and WLAN Systems, IEEE Transactions on Wireless Communications 11(7): 2658-2669.

Szott, S. 2014. Selfish Insider Attacks in IEEE 802.11s Wireless Mesh Networks, IEEE Communications Magazine, 52(6): 227-233.

Tan, K.; Song, J.; Zhang, Q.; Sridharan, M. 2006. A Compound TCP Approach for Highspeed and Long Distance Networks, in Proc. IEEE INFOCOM 2006. 25TH IEEE Int. Conf. on Computer Commun., Barcelona, Spain, 2006, 1-12.

Texas Instrument. 2003. WLAN channel bonding: Causing Greater Problems Than It Solves, White Paper SPLY0033-Sept, 2003, 12 p.

Tinnirello, I.; Giustiniano, D.; Scalia, L.; Bianchi, G. 2009. On the side-effects of proprietary solutions for fading and interference mitigation in IEEE $802.11 \mathrm{~b} / \mathrm{g}$ outdoor links, Computer Networks 53(2): 141-152.

Tuysuz, M. F.; Mantar, H. A.; Celik, G.; Celenlioglu, R. 2013. A Novel Beacon-based Collision-Free Channel Access Mechanism over IEEE 802.11 WLANs, Wireless Communications and Networking Conference (WCNC), 2013, 374-379. 
Ubnt Rocket M5 and Ubnt Powerbeam AC https://dl.ubnt.com/datasheets/rocketm/RocketM_DS.pdf

Urushihara, T.; Takahashi, H.; Kobayashi, M.; Motozuka, H.; Irie, M.; Shirakata, N.; Takinami, K. 2014. 60GHz wireless technologies for WiGig/IEEE 802.11ad multi-gigabit systems, Microwave Conference (APMC), 2014, 628-630.

Wang, B.; Wu, Y.; Liu, R. 2010. Game theory for cognitive radio networks: an overview, Computer Networks, The Int. J. of Computer and Telecommunications Networking 54(14): 2537-2561.

Wang, X.; Ren, Y.; Zhao, J.; Guo, Z.; Yao, R. 2004. Comparison of IEEE 802.11e and IEEE 802.15.3 MAC. Emerging Technologies: Frontiers of Mobile and Wireless Communication, 2004. Proceedings of the IEEE 6th Circuits and Systems Symposium on, 2004, 675-680.

Wang, Z.; Zou, W.; Du, G. 2014. A reliable MAC layer multicast protocol in Millimetre Wave WPANs, Communication Problem-Solving (ICCP), 2014 IEEE International Conference on, 2014, 169-172.

WG802.11-Wireless LAN Working Group, in IEEE P802, ed. by. 11ac Draft 7.0: Enhancements for Very High Throughput for Operation in Bands Below 6 GHz. Nov. 2013, $1-446$.

Ruckus. Getting the Best Wi-Fi Performance Possible through Both Beamforming and Adaptive Antennas, 2013, 1 p.

Więcek, D.; Wypiór, D. 2013. New SEAMCAT Propagation Models: Irregular Terrain Model and ITU-R P. 1546-4, Journal of Telecommunications and Information Technology, 2011, 131-140.

Xuelin, C.; Zuxun, S.; Bo, Y. 2015. TR-MAC: A Multi-step Slot Reservation-based Hybrid MAC Protocol for Ad Hoc Networks, Signal Processing, Communications and Computing (ICSPCC), 2015 IEEE International Conference on, 2015, 1-5.

Zhang, J.; Han, G.; Qian, Y. 2016. Queuing theory based co-channel interference analysis approach for high-density wireless local area networks, Sensors 16(9): 1-17.

Zhang, J.; Shen, H.; Tan, K.; Chandr, R.; Zhang Y.; Zhang, Q. 2012. Frame Retransmissions Considered Harmful: Improving Spectrum Efficiency using Micro-ACKs, Proc. Mobicom 2012, 22-26 August 2012, 1-12.

Zhang, X.; Lu, L.; Funada, R.; Sum, S.; Harada, H. 2010. Physical layer design and performance analysis on multi-Gbps millimeter-wave WLAN system, Communication Systems (ICCS), 2010 IEEE International Conference on, 2010, 92-96.

Zhong, Z.; Cao, F.; Kulkarni, P.; Fan, Z. 2016. Promise and perils of Dynamic Sensitivity control in IEEE 802.11 ax WLANs, IEEE ISWCS, 2016, 439-444.

Zhong, Z.; Kulkarni, P.; Cao, F.; Fan, Z.; Armour, S. 2015. Issues and challenges in dense WiFi networks, IEEE IWCMC 2015, pp. 947-951, 2015, 947-951. 
Zhou, Wei; Nandagopalan, S; Qiao, D. 2009. A Simulation Study of CSMA/CA Performance in $60 \mathrm{GHz}$ WPANs, Global Telecommunications Conference, 2009. GLOBECOM 2009, 1-6.

Zhu, B.; Kang G.; Zhang, N.; Liu, X.; Li, C.; Liu, Y. 2014. Enhanced block ACK in Distributed Coordination Function for next generation network, Communications and Networking in China (CHINACOM), 2014 9th International Conference on, 2014, 499-503.

Zhu, X.; Doufexi, A.; Kocak, T. 2012. A performance enhancement for $60 \mathrm{GHz}$ wireless indoor applications, Consumer Electronics (ICCE), 2012 IEEE International Conference on, 2012, 209-210.

Zhu X.; Doufexi, A.; Kocak, T. 2011. A performance evaluation of 60 GHz MIMO systems for IEEE 802.11ad WPANs, Personal Indoor and Mobile Radio Communications (PIMRC), 2011 IEEE 22nd International Symposium on, 2011, 950-954. 


\section{List of Publications by the Author on the Topic of the Dissertation}

\section{Papers in the Reviewed Scientific Journals}

Cuzanauskas, T.; Haddad, Y. 2017a. A New Game for Future Wi-Fi Spectrum Sharing, Elektronika ir elektrotechnika 23(3): 89-95, ISSN 1392-1215, (Clarivate Analytics Web of Science).

Cuzanauskas, T.; Anskaitis, A. 2016a. TCP performance in multi-polling game theorybased IEEE 802.11 networks, Electrical, control and communication engineering 11(1): 33-40, ISSN 2255-9140.

Cuzanauskas, T.; Anskaitis, A. 2016b. Innovative wireless medium control algorithm for wireless devices in ISM bands. International Journal of Computer Applications (IJCA) 146(6): 1-5 ISSN 0975-8887.

Cuzanauskas, T.; Anskaitis A. 2015a. Žaidimų teorijos taikymas IEEE 802.11 tinkluose. Mokslas-Lietuvos ateitis : elektronika ir elektrotechnika = Science-future of Lithuania: electronics and electrical engineering 7(3): 345-349, ISSN 2029-2341. 


\section{Papers in Other Editions}

Cuzanauskas, T.; Anskaitis, A. 2016c. IEEE 802.11e capabilities in dense wireless networks. 2016 Open Conference of Electrical, Electronic and Information Sciences (eStream), April 19, 2016, Vilnius, Lithuania / Vilnius Gediminas Technical University. New York: IEEE, 2016, 1-4, ISBN 9781509015160.

Cuzanauskas, T.; Anskaitis, A. 2015b. Multi-polling game for IEEE 802.11 networks. AIEEE 2015. Advances in Information, Electronic and Electrical Engineering (AIEEE) : proceedings of the 2015 IEEE 3rd workshop, November 13-14, 2015 Riga, Latvia. New York: IEEE, 2015. 1-5, ISBN 9781509012015.

Cuzanauskas, T.; Anskaitis, A. 2015c. Game theory for future IEEE 802.11 spectrum sharing. Electrical, Electronic and Information Sciences (eStream) : proceedings of the 2015 Open conference, 21 April, 2015, Vilnius, Lithuania/Vilnius Gediminas Technical University. New York: IEEE, 2015, 1-6, ISBN 9781467374453.

Cuzanauskas T.; Medeišis, A.; Anskaitis, A. 2015c. Activity dependent power allocation algorithm for IEEE 802.11 networks. 11th IEEE International Wireless Communications and Mobile Computing Conference (IEEE IWCMC), 24-28 Aug., 2015, Dubrovnik, Croatia : [proceedings]. New York: IEEE, 2015, 79-83, ISSN 2376-6492.

Medeišis A.; Sydor J.; Cremene, L.; Holland, O.; Anskaitis, A.; Wiecek, D.; Haddad, Y.; Cuzanauskas, T. 2014a. ISM-Advanced: Improved access rules for unlicensed spectrum. 2014 IEEE International symposium on dynamic spectrum access networks (DySPAN 2014), April 1-4 2014, McLean, Washington, USA. Washington: IEEE, 2014, 194-205, ISSN 2334-3125.

Cuzanauskas, T.; Medeišis, A.; Anskaitis, A; Cremene, L; Sydor, J; Holland, O.; Haddad, Y.; Nekovee M. 2014b. Interference-aware power coordination game for ISM bands. 9th International conference on cognitive radio oriented wireless networks and communications (CROWNCOM), 2-4 June, 2014, Oulu, Finland. New York: IEEE, 2014, 389394, ISSN 2166-5370.

Medeišis, A.; Sydor, J.; Cremene, L.; Więckowsk, D.; Haddad, Y.; Holland, O.; Anskaitis, A.; Cuzanauskas, T. 2014c. ISM-advanced band concept. Cognitive radio policy and regulation. Amsterdam: Springer Netherlands, 2014, 357-367, ISBN 9783319040226.

Cuzanauskas, T. 2017b. Hybrid rate control mechanisms in IEEE 802.11 networks. 2017 Open conference of Electrical, Electronic and Information Sciences (eStream) : proceedings of the conference, April 27, 2017, Vilnius, Lithuania / Organized by: Vilnius Gediminas technical university. New York: IEEE, 2017, 1-4, ISBN 9781538639986. 


\section{Summary in Lithuanian}

\section{Ivadas}

\section{Problemos formulavimas}

Bevielès ịrangos IEEE 802.11 paplitimas tapo įmanomas dèl supaprastintų naudojimo taisyklių, mažos kainos ir nelicencijuoto spektro ruožo, kuris išskirtas šiai bevielei technologijų grupei ("Authorization of Spread Spectrum Systems Under Parts 15 and 90 of the FCC Rules and Regulations". Federal Communications Commisions, USA, June 18, 1985). Tai lèmé eksponentini Wi-Fi ịrangos augimą ir platų pritaikymą ịvairiose srityse (Report for the European Commision 2013). Tačiau augantis populiarumas ir lanksčios taisyklès, kurios nenurodo, kaip ịranga turi būti diegiama ar naudojama, lèmè bendrą perdavimo spartos sumažejimą. Didelio tankio Wi-Fi tinklai ir nekontroliuojamas įrangos naudojimas, tapo duomenų perdavimo spartos sumažẻjimo priežastimi.

Standartizuotos IEEE 802.11 radijo terpès prieigos schemos tapo neefektyvios ir nebegali garantuoti stabilumo ir bendros sistemos spartos šiuolaikiniuose Wi-Fi tinkluose, kurie dèl didelio vartotojų skaičiaus tapo dar labiau perkrauti. (Chatzimisios et al. 2004; Baid et al. 2015; Zhang et al. 2016). Viena iš priežasčių, kuri lėmè tokị spartos mažèjimą, tai ribotas nelicencijuotas radijo spektro ruožas ir ịrenginiu tarpusavio koordinavimo funkcijos nebuvimas sukeliantis duomenų perdavimo trukdžius (Lee et al. 2016, Zhang et al. 2016).

Siekiant spręsti Wi-Fi ịrangos našumo problemas buvo atliekami tyrimai akademineje aplinkoje ir privačiose įmonèse, kurios gamina IEEE 802.11 bevielius įrenginius. Kai kurių tyrimų metu našumo problemą buvo bandoma spręsti jau standartizuoto protokolo 
pakeitimais. Vienas iš galimų pakeitimų, tai radijo terpès prieigos funkcijos MAC (angl. Media Access Control) parametru keitimas esant skirtingoms radijo eterio sąlygoms.

Kita populiari tyrimo sritis, tai laikinio eterio dalinimo modelio pritaikymas (angl. Time Division Multiple Access), kuris pakeistų paskirstytaji koordinavimo rěžimą (angl. Distributed Coordination Function). Tačiau, nevisi sprendimai buvo įdiegti ịrenginiuose dèl suderinamumo problemų. Dalis pasiūlytų pakeitimų lèmė dar didesnị spartos sumažèjimą.

Rinkoje pasirodęs naujasis IEEE 802.11ac protokolas turejjo spręsti dalị išvardintų problemų. IEEE $802.11 \mathrm{ac}$ pristate aukštesnès moduliacijos galimybès ir platesnius $80 \mathrm{MHz}$ ir $160 \mathrm{MHz}$ kanalo pločius, kurie leido padidinti perdavimo spartą. Tačiau didesni kanalų pločiai ir beveik nepakitęs radijo terpès prieigos rěžimas sudare sąlygas tolimesniam spartos mažèjimui dèl didèjančios interferencijos (Hanna, Sydor 2012; Lee et al. 2016, Zhang et al. 2016). Todel reikalingas IEEE 802.11 MAC lygmens pakeitimas, kuris leistų efektyviau išnaudoti turimą nelicencijuotą radijo dažnių ruožą ir užtikrintų augančius vartotojų poreikius.

Siekiant išnagrinèti ịvardintą problemą, iškelta hipotezė: IEEE 802.11 MAC pakeitimas gali pagerinti IEEE 802.11 duomenų perdavimo pralaidumą, kai aptarnaujamas didelis kiekis prisijungusių klientų lyginant su IEEE 802.11 EDCA ir IEEE 802.11 PCF sprendimais.

\section{Darbo aktualumas}

IEEE 802.11 bevieliai ịrenginiai dèl savo mažos kainos tapo viena populiariausių technologijų duomenims perduoti. Kas lèmè tolimesnį Wi-Fi ịrangos plètojimą ir naujų standartų atsiradimą. Tačiau esami patobulinimai negarantuoja patikimo Wi-Fi įrangos veikimo esant dideliam vartotoju skaičiui. Šio neefektyvumo priežastis yra ribotas radijo kanalo prieigos valdymo mechanizmas, kuris išliko nepakitęs nuo pirmojo standarto atsiradimo, o laikui bègant buvo sukuriami tiktai papildomi patobulinimai, kurie nepakeite pagrindinio IEEE 802.11 MAC lygmens veikimo. Šiuo metu IEEE 802.11 yra paremtas vienu pagrindiniu kanalo valdymo algoritmu - paskirstytojo koordinavimo réžimu (angl. Distributed Coordination Function) DCF yra vienintelè, kuri buvo sertifikuota Wi-Fi aljanso. Dẻl šios priežasties dauguma Wi-Fi įrenginių naudoja būtent nurodytą įrenginių koordinavimo funkciją. Nors paskirstyta kanalo prieiga yra efektyvi, esant ribotai duomenų perdavimo spartai, kuri užtikrina žemą vèlinimą, tačiau esant didesniam stočị skaičiui tampa neefektyvi. Dèl augančių įrenginių skaičiaus, bendras radijo ryšio kanalo dalinimosi algoritmas tampa neefektyvus ir negali garantuoti pareikalautos duomenų perdavimo pralaidos. Radijo ryšio kanalo efektyvaus panaudojimo problema buvo ypač plačiai aptarta moksliniuose straipsniuose (Chatzimisios et al. 2004; Baid et al. 2016). Tačiau, didžioji dalis šiu tyrimų rezultatų, taip ir liko praktiškai neiggyvendinti dèl suderinamumo problemų su esama technologija bei dèl savo sudètingumo (Jardosh et al. 2005; Chandra et al. 2008, Gast et al. 2013).

Šioje disertacijoje atlikti teoriniai ir eksperimentiniai tyrimai, kurie detaliai išnagrinejo IEEE 802.11 MAC lygmens veikimą bei dabar esamų sprendimų perdavimo spartą. Remiantis gautais rezultatais buvo pasiūlytas ir igyvendintas naujas IEEE 802.11 MAC 
lygmens pakeitimas, kuris užtikrintų didesnę duomenų perdavimo spartą didèjant vartotojų skaičiui. Siekiant tai padaryti, buvo pasiūlytas aktyvių ir neaktyvių klientų srautų valdymo algoritmas.

Siekiant pagerinti IEEE 802.11 MAC lygmens našumą buvo tiriamas ir papildomas žaidimų teorija pagrissto galios valdymo modelis.

Atliekant teorinius ir eksperimentinius tyrimus, buvo sukurtos bevielių tinklų topologijos, kurios leistų ịvairiapusiškai išbandyti pateiktą MAC lygmens modifikaciją.

\section{Tyrimų objektas}

Pagrindinis tyrimų objektas, tai IEEE 802.11 kanalo prieigos gerinimo būdai, kurie leistų padidinti sistemos perdavimo spartą esant dideliam įrenginių skaičiui.

\section{Darbo tikslas}

Disertacijos tikslas - teoriškai ir praktiškai ištirti bei pasiūlyti efektyvią belaidžio ryšio IEEE 802.11 MAC lygmens pakeitimą, kuris leistų padidinti IEEE 802.11 sistemos perdavimo spartą sutankintuose tinkluose.

\section{Darbo uždaviniai}

Darbo tikslui pasiekti sprendžiami šie uždaviniai:

1. Atlikti teorinė ir eksperimentinę IEEE 802.11 kanalo prieigos analizę.

2. Pasiūlyti IEEE 802.11 MAC lygmens pakeitimus, kurie pagerintų sistemos perdavimo spartą, taškas keli taškai tinklo topologijose.

3. Pagristi pateikto IEEE 802.11 MAC lygmens pakeitimo efektyvumą eksperimentiniais matavimais ir palyginamąja analize.

4. Pasiūlyti kelių dažnių sistemos pakeitimą, kuris leistų padidinti IEEE 802.11 ad perdavimo spartą esant aktyviems ir neaktyviems klientų srautams naudojant FST rèžimą.

\section{Tyrimų metodika}

Siekiant ištirti pagrindinị disertacijos tyrimų objektą bei išspręsti užsibrėžtus uždavinius, bus taikoma ši tyrimų metodika:

- Klasifikavimas - atliekama literatūros analizė iš kurios nustatomas tyrimų objektas

- Hipotezè - atliekama teorinè ryšio kanalo prieigos analize

- Statistinè analizè - atliekama teorinè IEEE 802.11 sistemos analizè remiantis literatūra bei atliekant modeliavimus.

- Eksperimentiniai tyrimai - atliekami naudojant IEEE 802.11 ịrangą, skirtinguose scenarijuose siekiant ịvertinti ir pagrịsti siūlomos modifikacijos efektyvumą. 


\section{Darbo mokslinis naujumas}

Atlikus teorinius ir praktinius bandymus buvo gauti šie elektros ir elektronikos inžinerijos mokslui nauji rezultatai:

1. Sukurtas naujas ryšio kanalo paskirstymo algoritmas ir MAC lygmens architektūriniai sprendimai, kurie leidžia padidinti duomenų perdavimo spartą IEEE 802.11 taškas keli taškai bevielio tinklo topologijoje.

2. Igyvendintas naujas siųstuvo galios valdymo algoritmo pritaikymas taikant žaidimų teoriją IEEE 802.11 tinkluose. Rezultatai bei modeliai gali būti pritaikyti tolimesniam funkcionalumo plètojimui.

3. Gauti nauji eksperimentiniai matavimo rezultatai, nustatantys paslèptos stoties ịtaką IEEE 802.11 tinkluose bei nurodantys būdus spręsti įrenginių tarpusavio kolizijas.

4. Gauti nauji eksperimentiniai matavimo rezultatai, nusakantys TDMA sistemos efektyvumą

5. Gauti nauji eksperimentinių matavimų rezultatai naudojant IEEE 802.11ad $60 \mathrm{GHz}$ sistemą, kurie nustato šios sistemos efektyvumą taškas keli taškai bevielio tinklo topologijose.

\section{Darbo rezultatų praktinè reikšmè}

Rengiant disertaciją gauti šie praktiniai rezultatai:

1. MAC lygmens architektūriniai sprendimai, leidžia efektyviau išnaudoti eterio laiką, kelių stočių aptarnavimui lyginant su IEEE 802.11 EDCA ir IEEE 802.11 PCF sprendimais.

2. Kelių dažnių sistemos standarto apibrèžto funkcionalumo praplètimas užtikriną didesnę perdavimo spartą. Pasiūlytas papildomas FST funkcionalumas leidžia efektyviau išnaudoti $5 \mathrm{GHz}$ ir $60 \mathrm{GHz}$ dažnių sistemas.

3. Remiantis disertacijose aprašytais teoriniais ir praktiniais rezultatais, buvo sukurta sistema komerciniuose produktuose, kurie veikia IEEE 802.11 tinkluose ir yra pardavinëjami ịvairiose šalyse.

\section{Darbo rezultatų aprobavimas}

Disertacijos tema paskelbta 12 mokslinių straipsnių. Vienas straipsnis Clarivate Analytics Web of Science duonenų bazejje referuojamuose mokslo žurnaluose su citavio indeksu, 3 straipsniai - kituose recenzuojamuose mokslo leidiniuose, aštuoni - Clarivate Analytics Web of Science duonenų bazėje „Conference Proceedings“. Perskaityti aštuoni pranešimai tarptautinèse konferencijose:

1. Tarptautinè konferencija „Advances in Information, Electronic and Electrical Engineering 2013“. 2013. Riga, Latvia.

2. Tarptautinè konferencija ,IEEE International symposium on dynamic spectrum access networks 2014". 2014. McLean, Washington, USA.

3. Tarptautine konferencija "9th International conference on cognitive radio oriented wireless networks and communications 2014”. 2014. Oulu, Finland. 
4. Tarptautinè konferencija ,Advances in Information, Electronic and Electrical Engineering 2015 “. 2015. Riga, Latvia.

5. Tarptautinè konferencija ,Electrical, Electronic and Information Sciences 2015 “. 2015. Vilnius, Lithuania.

6. Tarptautinè konferencija ,International Wireless Communications and Mobile Computing Conference 2015“. 2015. Dubrovnik, Croatia.

7. Tarptautine konferencija „Electronic and Information Sciences 2016“. 2016, Vilnius, Lithuania.

8. Tarptautinè konferencija „Electronics 2017”. 2017. Palanga, Lithuania.

Disertacijos rezultatai buvo panaudoti pateikiant bendrą Lietuvos ir Lenkijos universitetų „Daina“ projektą, kuris apibrèžia naujas gaires IEEE 802.11 funkcionalumui.

\section{Ginamieji teiginiai}

1. Pasiūlytas ir ịgyvendintas naujas IEEE 802.11 MAC lygmens algoritmas leidžia padidinti duomenų perdavimo spartą atitinkamai: iki $12 \%$, kai aktyvių įrenginių skaičius yra 13 lyginant su IEEE 802.11 PCF (be varžymosi periodo) ir iki $79 \%$ lyginant su IEEE 802.11 EDCA. Kai aktyvių įrenginių skaičius siekia 60, MP protokolas duomenu pralaidumą padidina iki $388 \%$ lyginant su EDCA ir iki 50 \% lyginant su PCF, kai siunčiamas dvikryptis TCP srautas.

2. Naujas IEEE 802.11 MAC lygmens algoritmas leidžia išgauti iki $89 \%$ didesnę spartą siunčiant dvikryptị TCP srautą, lyginant su dabar rinkoje siūlomu TDMA sprendimu, kai yra tiktai vienas aktyvus klientas ir naudojamas lankstus TDMA laiko intervalas

3. Aktyvių ir neaktyvių klientų paskirstymo algoritmas leidžia sumažinti vèlinimą iki 12 kartų lyginant su IEEE 802.11 PCF (be varžymosi periodo) sprendimu, kai prisijungusių vartotojų skaičius yra daugiau negu 60.

4. Aktyvių ir neaktyvių klientų skirstymas $5 \mathrm{GHz}$ ir $60 \mathrm{GHz}$ sistemoje gali padidinti duomenu perdavimo spartą iki $18 \%$ lyginant su ịprastine taškas keli taškai $60 \mathrm{GHz}$ tinklo topologija, kai visi klientai aktyviai siunčia duomenis.

\section{Disertacijos struktūra}

Disertaciją sudaro įvadas ir 3 pagrindiniai skyriai, bendrosios išvados, šaltinių ir literatūros sąrašas, autoriaus mokslinių publikacijų disertacijos tema sąrašas ir santrauka lietuvių kalba. Disertacijos apimtis 162 puslapiai. Disertacijoje pateikta 51 paveikslas, 9 lentelès, 10 numeruotų formulių ir panaudoti 152 literatūros šaltiniai.

\section{IEEE 802.11 iššūkiai ir pažanga bevieliuose tinkluose}

IEEE 802.11 yra viena populiariausių bevielių technologijų duomenims perduoti, kurios populiarumą lèmė nedidelè kaina ir lanksčios naudojimo sąlygos. Tačiau augantis populiarumas ir šios technologijos pritaikymas ịvairiose srityse lèmė ryšio kokybės sutrikimus. 
Kadangi IEEE 802.11 veikia nelicencijuotame dažnyje, jis tapo perpildytas ir dèl šios priežasties krito bendras sistemos pralaidumas. Siekiant spręsti susidariusią problemą, atliekami tyrimai tiek akademinėje aplinkoje tiek ir komercinėse įmonėse. Daugelių tyrimų metu pagrindinis tyrimo tikslas yra efektyvesnis MAC lygmuo, kuris užtikrintų didesnị sistemos pralaidumą. Dalis tyrëjų susikoncentruoja ị specifines sritis, tokias kaip TCP protokolo tyrimas bevieliuose tinkluose (Pavilanskas 2007; Brouer, Hansen 2004). Dali kilusių problemų bandoma spręsti naudojant RTS/CTS (Chou et al. 2013). Sudètingesnès modifikacijos atliekamos visiškai pakeičiant MAC lygmens protokolą, tam kad igyvendinti specifinius poreikius arba tiksliau kontroliuoti klientų ryšio kanalo rezervavimą (Fallah, Alnuweiri 2007; Leffler 2009). Tačiau, dalis šių modifikacijų nèra suderinama su dabar naudojamais ịrenginiais ir tai lemia nekontroliuojamą radijo kanalo prieigos valdymą.

Analizuojant literatūrą nustatytas nepakankamas tyrimas bendrų komponentų visumos efektyvesniam radijo ryšio kanalo prieigos valdymui, kai aptarnaujamas didelis bevielių stočių skaičius, kuris dažnai sutinkamas tarp interneto paslaugų tiekèjų. Dèl augančių vartotojų paklausos, bevielès ryšio ịrangos sprendimai nebeužtikrina ryšio stabilumo ir pralaidos reikalavimų. Tai lemia ne tik neefektyvus radijo kanalo resursų panaudojimas, bet ir siųstuvo galios ribojimas, kuris sumažina ryšio kanalo kokybinius parametrus. Todèl tinkamas siųstuvo galios reguliavimas yra svarbus siekiant užtikrinti interferencijos kontrolę.

Pastebima tendencija, kad ryšių reguliavimo tarnybos siekia išplèsti nelicencijuotų dažnių juostą. Tai leidžia padidinti bendrą IEEE 802.11 aprèptị bei pagerinti kokybinius parametrus. Tačiau, tam kad tinkamai išnaudoti naujus nelicencijuotus dažnius reikalingas efektyvesnis IEEE 802.11 MAC lygmuo, kuris spręstų duomenų srautų valdymo problemą. Siekiant išspręsti išsikeltą IEEE 802.11 MAC lygmens efektyvumo problemą, pagrindinè hipotezė buvo iškelta ir įrodyta:

IEEE 802.11 MAC modifikacija gali pagerinti IEEE 802.11 sistemos pralaidumą, kai aptarnaujamas didelis kiekis prisijungusių klientų lyginant su IEEE 802.11 EDCA ir IEEE 802.11 PCF (be varžymosi periodo) sprendimais.

Yra keletas ryšio perdavimo terpès valdymo protokolų MAC (angl. Media Access Control) rūšių. Labiausiai paplitęs, tai yra EDCA (angl. Enhanced Distributed Channel Access), kuriame naudojama kolektyvioji nešlio jutimo kreiptis be susidūrimų CSMA/CA (angl. Carrier Sense Multiple Access/Collision Avoidance). Tokių būdu siekiama užtikrinti, kad neįvyktų kolizijos tarp kanale veikiančių ịrenginių. Be EDCA, kitas IEEE 802.11 siūlomas valdymo protokolas, tai centralizuoto koordinavimo rèžimas PCF (angl. Point Coordinated Function). Šis radijo resursų valdymo réžimas yra mažai paplitęs ir praktiškai nenaudojamas. Tuo tarpu IEEE 802.112016 metų standartas siekia išmesti ši prieigos protokolą ir naudoti tiktai hibridinị radijo terpès koordinavimo réžimą HCF (angl. Hybrid Coordination Function). HCF yra EDCA ir PCF protokolų kombinacija, tačiau pats protokolas orientuotas ị stotis, kurios perduoda aukšto prioriteto duomenis, tuo tarpu stotys kurios perduoda žemo prioriteto duomenis vis dar veikia pagal EDCA taisykles. Pagrindinis skirtumas tarp PCF ir EDCA yra tai, kad prieigos taškas AP kontroliuoja prisijungusius įrenginius, kurie gali perduoti duomenis tiktai gavus kontrolinị paketą (angl. Token). Tuo tarpu EDCA paskirstytoji DCF funkcija leidžia dalintis fiziniu kanalu naudojant CSMA/CA. 
Kiekviena stotis prieš perduodama duomenis atlieka privalomą perdavimo kanalo pasiklausymą ir nustato ar perdavimo kanalas yra užimtas. Jeigu kanalas užimtas stotis sustabdo duomenų perdavimą ir atsitraukia atsitiktiniam laiko tarpui BEB (angl. Binary Exponential Back-off). Be šių pagrindinių MAC lygmens protokolų egzistuoja optimizuotos IEEE 802.11 standarto versijos, kurios veikia tuose pačiuose dažniuose kaip ir ịprasti Wi-Fi ịrenginiai. Tokie ịrenginiai ryšio kanalo eterị valdo TDMA (angl. Time-Division Multiple Access) pagrịstu protokolu. Šio protokolo esmè, kad kiekviena stotis gauna išskirtą laiko tarpą, kada gali būti perduoti duomenys, o laiko tarpų paskirstymą atlieka prieigos taškas.

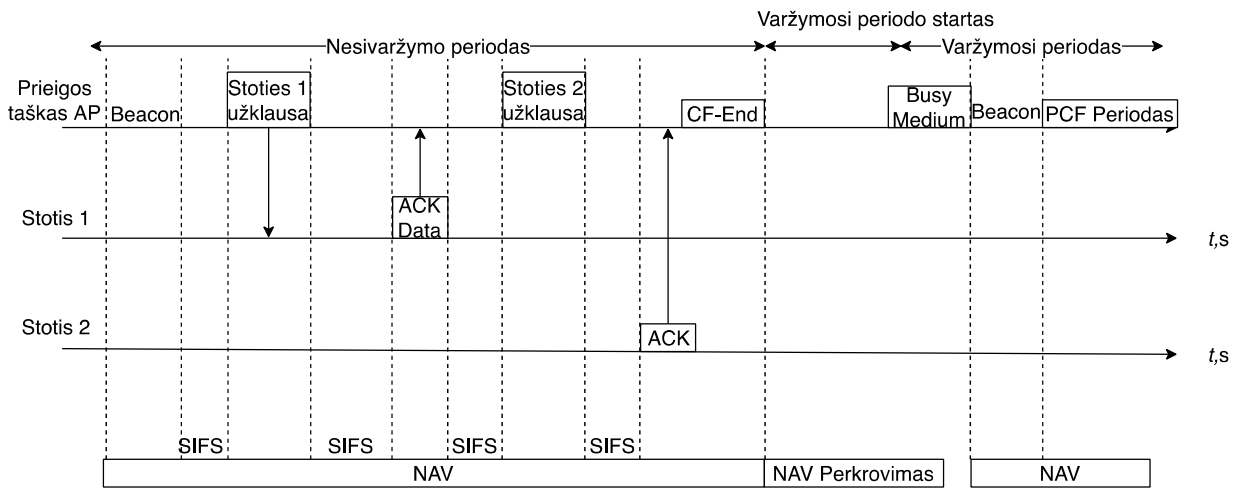

S1.1 pav. IEEE 802.11 centralizuoto koordinavimo rèžimo (angl. Point Coordination Function) paskirstymo schema

S1.1 paveiksle pavaizduotas PCF protokolo veikimas. Šiuo atveju, AP turi du klientinius ịrenginius, kiekviena stotis duomenis gali perduoti tiktai tada, kada gauna kontrolini užklausos paketą. IEEE 802.11 PCF periodas pradedamas su CF-Start (angl. Contention Free Start) paketo išsiuntimu. Visą radijo kanalo kontrolę šiuo atveju atlieka prieigos taškas, kuris sumažiną paslèptos stoties įtaką bendram sistemos našumui. Pagrindinis PCF protokolo trūkumas tas, kad nepriklausomai nuo duomenų kiekio visada vyksta aktyvus prijungtų stočių užklausimas. Tai lemia mažesnę duomenų perdavimo spartą, kai neaktyvių stočių kiekis didèja.

S1.2 paveiksle nurodyta HCCA kanalo valdymo schema, kuri kitaip nei PCF išskiria kelis stočių aptarnavimo intervalus. HCCA atveju perdavimo kanalas skirstomas ị dvi dalis: varžymosi periodą (angl. Contention Period) ir kontroliuojamą periodą (angl. Contention Free Period). Kiekvienas iš prisijungusių įrenginių prie AP yra kontroliuojamas, tam išskiriant nustatytą trukmę duomenims perduoti. Tai vykdoma naudojant CF-Poll (angl. Contention Free Poll) užklausos paketus. Papildomai HCCA rezervuoja eterị, nustatydamas laiko parametrą NAV (angl. Network Allocation Vector) laukelyje, kuris nurodo kiek laiko kanalas bus rezervuojamas duomenų perdavimui. Toks ryšio kanalo valdymo būdas užtikrina, kad kitas įrenginys nebandys perduoti duomenų tuo pačiu metu. Kontroliniai CAP paketai (angl. Controlled Access Period) skirti perimti kanalo duomenų perdavimui, šiuos kontrolinius paketus išskirtinai naudoja AP, kai reikia aptarnauti aukštesnio prioriteto srautus varžymosi periodo metu. 


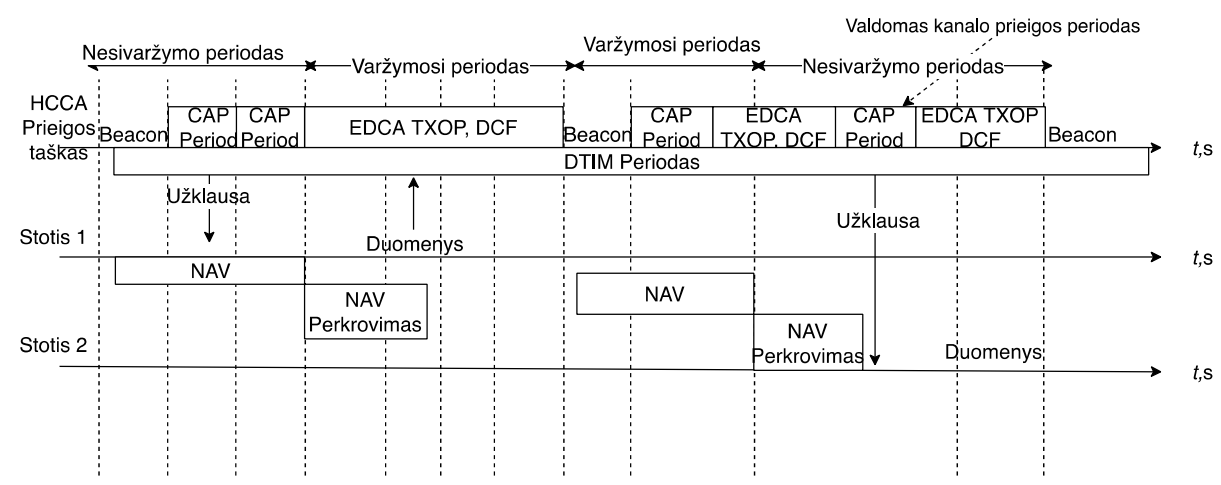

S1.2 pav. IEEE 802.11 hibridinès kanalo prieigos paskirstymo schema

Nors HCCA pasižymi patobulintu eterio valdymo mechanizmu, tačiau jis vis dar turi tokių pat trūkumų kaip ir PCF protokolas. Varžymosi periodo metu, galimi duomenų paketų praradimai tarp bevielių įrenginių, todèl tiek PCF tiek HCCA negarantuoja, jog bus išvengta tarpusavio duomenų paketų praradimų. Dèl sudètingų technologinių sprendimų HCCA netapo komerciškai realizuotu sprendimu, vietoj šio ryšio kanalo dalinimosi algoritmo buvo pasirinktas EDCA sprendimas.

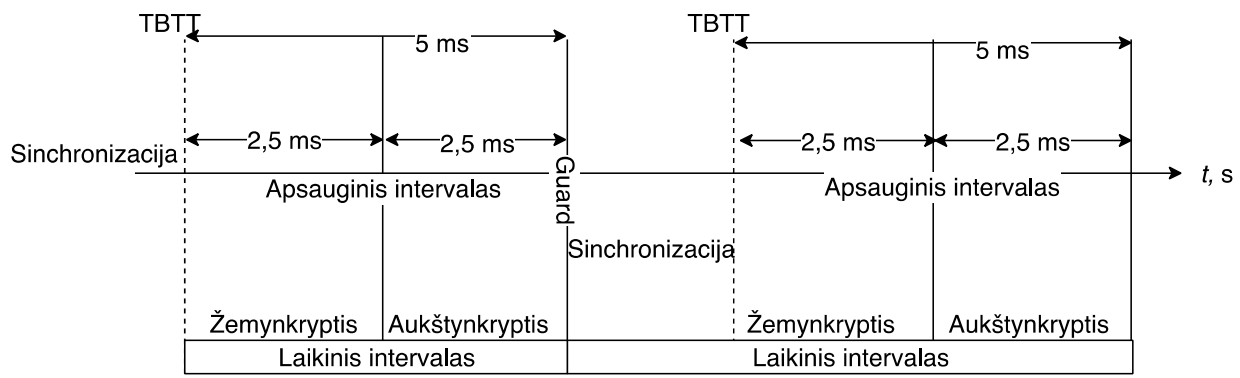

S1.3 pav. Laikinio kanalo prieigos superkadro struktūra

Be EDCA ir HCCA kanalo prieigos skirstymo schemų egzistuoja ir laikinė kanalo prieigos paskirstymo schema. TDMA yra viena populiariausių IEEE 802.11 protokolo schemų. S1.3 paveikslas nurodo bazinę TDMA protokolo kadro struktūrą, kuri apibrèžia žemynkrypčio ir aukštynkrypčio srauto laiko intervalus. Radijo kanalo kontrolè priklausomai nuo prisijungusių klientų aktyvumo gali būti dalinama ị skirtingus laiko intervalus. Kuo bevielis įrenginys siunčia mažiau duomenų, tuo labiau apribojama jo siuntimo laiko trukmè, kas leidžia padidinti kanalo valdymo efektyvumą.

Pagrindinis TDMA trūkumas, kad jis reikalauja sudètingų IEEE 802.11 MAC lygmens pakeitimų, kas lemia suderinamumo problemas su dabar egzistuojančiais ịrenginiais. Kadangi, TDMA griežtai valdo laiko intervalus - nenaudojamas CCA (angl. Clear Channel Assessment) algoritmas, tam kad ịvertinti ar ryšio kanalas neužimtas.

Dèl laikinès kontrolès TDMA išsiskiria būdinga paketų delsa. Priklausomai nuo TDMA schemos igyvendinimo galimi $2,5 \mathrm{~ms}, 5 \mathrm{~ms}$ ir $8 \mathrm{~ms}$ laiko intervalai (Leffler 2009), 
kurie suteikiami stotims duomenis perduoti ir gauti. Dèl duomenu perdavimo laiko apribojimo, TDMA tampa mažiau efektyvus lyginant su IEEE 802.11, kai aptarnaujamų irenginių skaičius siekia iki 5 .

TDMA protokolas leidžia visiškai atsisakyti standartizuoto IEEE 802.11 funkcionalumo, todèl ịdiegiant ši kanalo prieigos būdą, bevielès ịrangos gamintojai išjungia visus ryšio kanalo klausymosi algoritmus. Tuo siekiama pagerinti spektro išnaudojimo efektyvumą. Laikinis dalinimas naudingas dar ir tuo, kad kiekvienas klientas nepriklausomai nuo to kokia jo signalo kokybė yra apribotas laike, o ne paketu kiekyje kaip, IEEE 802.11 EDCA atveju. Tokiu būdu išvengiama sistemos spartos sumažejimo, kai AP aptarnauja klientus, kurie atlieka daug paketų pakartojimų dèl prastos ryšio kokybės.

\section{Teorinè keliụ dažnių bevielès sistemos analizè}

IEEE 802.11 MAC pakeitimai atlikti naudojant Qualcomm programinès įrangos paketą (angl. Software Development Kit). Tokiu būdu buvo sukurta kelių užklausų sistema MP (angl. Multi-Poll).

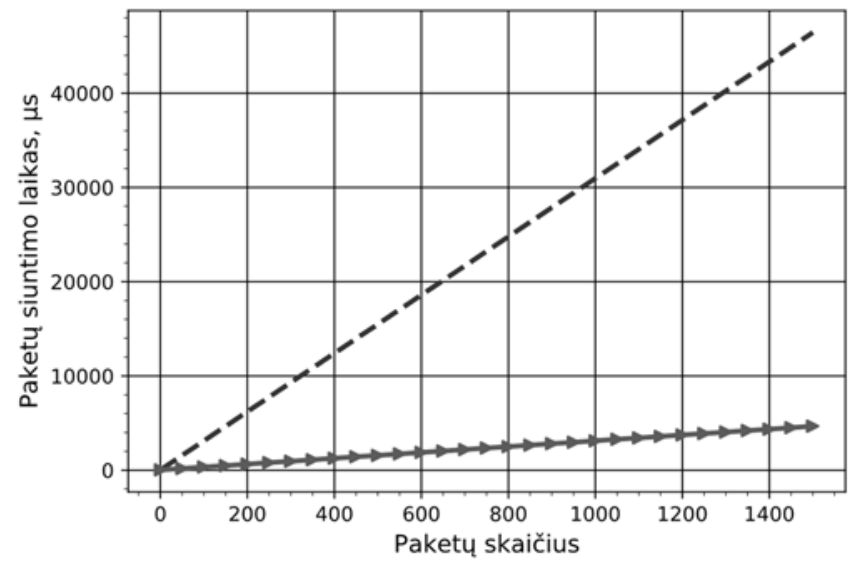

- - IEEE 802.11 GF eterio užimtumas su MCS0

IEEE 802.11 GF eterio užimtumas su MCS15

S2.1 pav. Eterio panaudojimo efektyvumas, esant skirtingai kontrolinių paketų moduliacijai

MP yra pagrịstas aktyvių ir neaktyvių klientų aptarnavimu EDCA CP (angl. Contention Period) periodo metu. Tai leidžia vienu metu aptarnauti aktyvius ir neaktyvius klientus. Kitaip negu PCF, MP (angl. Multi-Poll) nesiunčia papildomų kontrolinių užklausos paketų, jeigu klientas nèra aktyvus. Vietoje šių paketų, bazinè stotis esant žemam aktyvumui naudoja standartinị radijo kanalo prieigos būdą IEEE 802.11 EDCA. Bendra kanalo prieiga yra pagrissta EDCA funkcija, kuri naudojama aktyviame ir neaktyviame klientu srautu skirstyme. Kadangi, kitaip nei PCF ar HCCA MP protokolas kontroliniams paketams naudoja didelį duomenų perdavimo greitị (300 Mbps), kas lemia mažesnes laiko sąnaudas. Tai matyti iš S2.1 paveikslo, kuriame vaizduojama, kaip skiriasi ịprasto PCF kontrolinių paketų perdavimo trukmè, kai kontrolinių paketų skaičius didèja ir Multi-Poll atveju, kai naudojamas didelis kontrolinių paketų perdavimo greitis. 
MP protokolas naudoja nauja radijo stočiu paskirstymo algoritmą. Priklausomai nuo prisijungusio kliento aktyvumo, prieigos taškas AP atlieka aktyvią arba pasyvią radijo stočiu srautų kontrolę (žr. S2.2 pav.). MP atveju visi klientai veikia CP varžymosi periode, kuriame tuo pačiu vyksta ir aktyvių klientų srautų ribojimas, leidžiant jiems perduoti duomenis tiktai gavus užklausą (angl. Token).

Kitaip nei daugelis aptartų protokolų, kurie nagrinèjami straipsniuose, MP naudoja automatinę perdavimo greičio kontrolę, kuri yra kontroliuojama dinamiškai nuo prarastų paketų skaičiaus. Šis faktorius lemia, kad AP nepraranda perdavimo spartos esant nedideliam kolizijų skaičiui (S2.3 pav.).

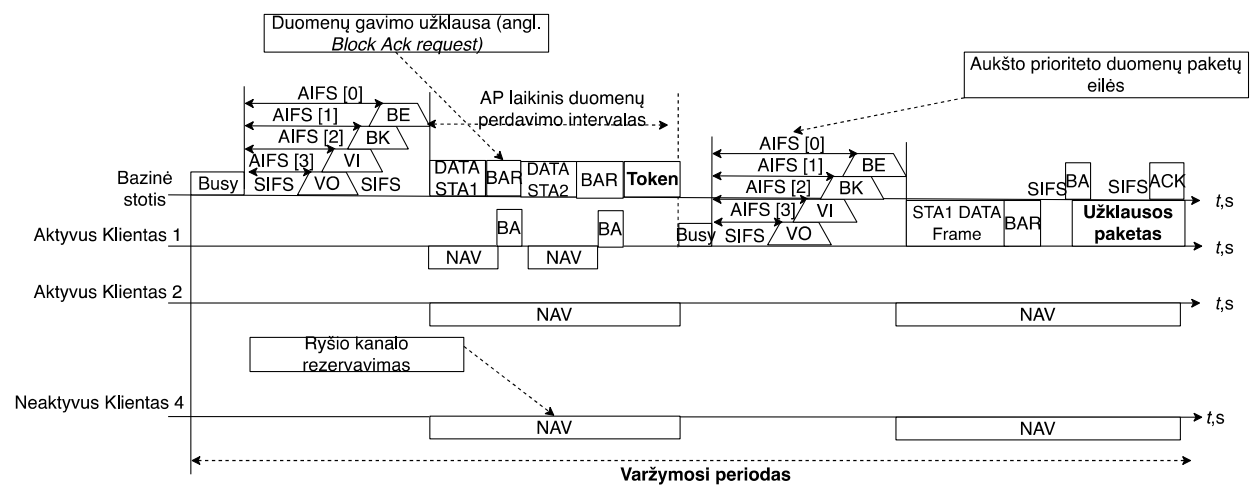

S2.2 pav. Multi-Poll kanalo prieigos schema neaktyviems klientams

Šiuo atveju bandymu metu buvo nustatyta paslèptos stoties srauto ịtaka bendram sistemos pralaidumui. Nustatytas didžiausias AP pralaidumas siekia iki $178 \mathrm{Mbps}$, kai nèra paslèptos stoties srauto. Tačiau atsiradus paslèptos stoties duomenų srautui, kuris viršija $5 \mathrm{Mbps}$, bendras sistemos pralaidumas mažèja. Augant paslëptų stočių skaičiui reikalinga papildoma kontrolè. Todèl, kai prieigos taškas aptarnauja didelị skaičių aktyvių ir neaktyvių klientų skaičių, turi būti atliekama dinaminè aktyvių ir neaktyvių klientų kontrolè. Ši kontrolė nustatoma keičiant klientų srauto ịvertinimo parametrus, tokius kaip perdavimo sparta bei perduotų paketų skaičius.

MP protokolas pasižymi tiek PCF tiek EDCA savybėmis, kai esant žemam srautui užtikrinamas mažas duomenų paketų vèlinimas, o esant dideliam srautui pradedamas aktyvus klientu apklausimas, kuris apriboja kolizijų skaičių ir leidžia išlaikyti sistemos spartą augant aktyvių klientų skaičiui. Kitaip negu TDMA, klientai nėra apriboti laike, todèl bendras kliento perdavimo našumas yra didesnis. Galimas trūkumas prie tam tikrų sąlygų, kad prastas signalo charakteristikas turintys klientai ịtakos bendrą sistemos spartą. Siekiant sukurti efektyvią IEEE 802.11 sistemą reikalingi kelių komponentų pakeitimai. Norint užtikrinti didesnę perdavimo spartą bei tinklo aprèptị reikalinga dinaminė siųstuvo galios kontrolè. Šiuo atveju, remiamasi žaidimų teorijos modeliu, kuris identifikuoja kiekvieną įrenginị, kaip individualų tinklo mazgą, sudarytą iš bazinių stočių. Toks pakeitimas leidžia efektyviau išnaudoti MP galimybes, kai užtikrinama tinkamas siųstuvo perdavimo galios valdymas. 


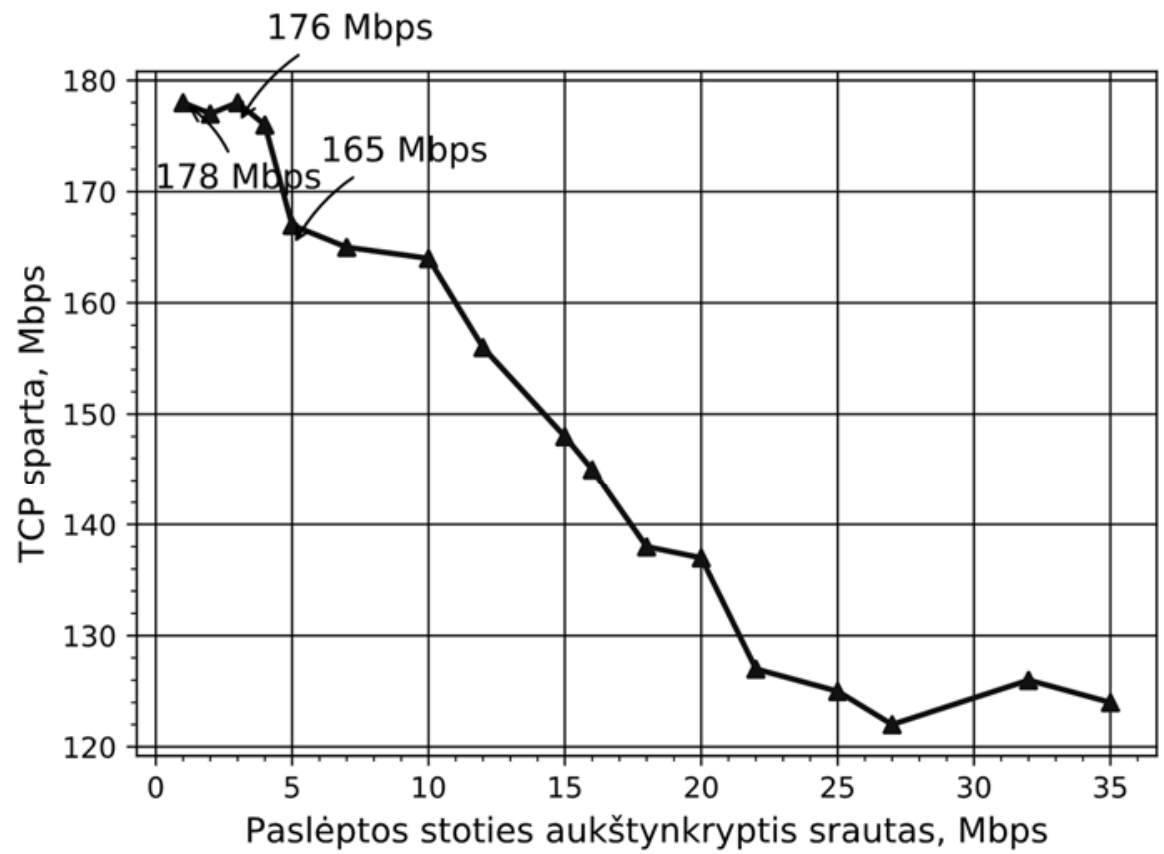

- $5 \mathrm{GHz}$ duomenu perdavimo sparta su paslèpta stotimi

S2.3 pav. Paslèptos stoties perdavimo spartos įtaka bendram sistemos pralaidumui

Žaidimų teorija pagrịsta teorija, jog kiekvieno ịrenginio tikslas pagerinti savo perdavimo spartą. Sparta gali kisti priklausomai nuo aplinkoje kitų ịrenginių siųstuvo perdavimo galios parametrų. Žaidimų teorija šiuo atveju leidžia dinamiškai valdyti įrenginių siųstuvų galią priklausomai nuo reikalaujamos spartos ar aplinkoje vykstančių pokyčių (Felegyhazi, Hubaux 2006; Benmammar, Krief 2014). Literatūroje aptariami keli žaidimų teorijos modeliai, kurie apibūdina paskirstytą galios valdymą (Wang et al. 2010; Kazemi et al. 2010). Kitaip nei ịprasti analitiniai modeliai, žaidimų teorija gali būti pritaikoma aplinkoje, kai sąlygos greitai kinta dèl kitų veikiančių įrenginių ar aplinkos sąlygų.

Norint įvertinti žaidimų teorijos modelį, buvo atlikti modeliavimai, kurie parodo, kad netgi uždaroje patalpoje, kurioje greta išdèstyti keli prieigos taškai, esant tinkamai galios kontrolei su didesne siųstuvo galia galima pasiekti didesnę perdavimo spartą. Tai matyti S2.4 paveiksle, jog pašalinus siųstuvo galios ribojimus pasiekiamas didesnis našumas, tačiau tuo pačiu vertinant vidutinę kiekvieno iš vartotojų perdavimo spartą matomas sumažèjimas, kas atspindi šių dienų Wi-Fi sistemose, kai augant vartotojų skaičiui kiekvieno iš vartotojų vidutinè perdavimo sparta mažèja. 

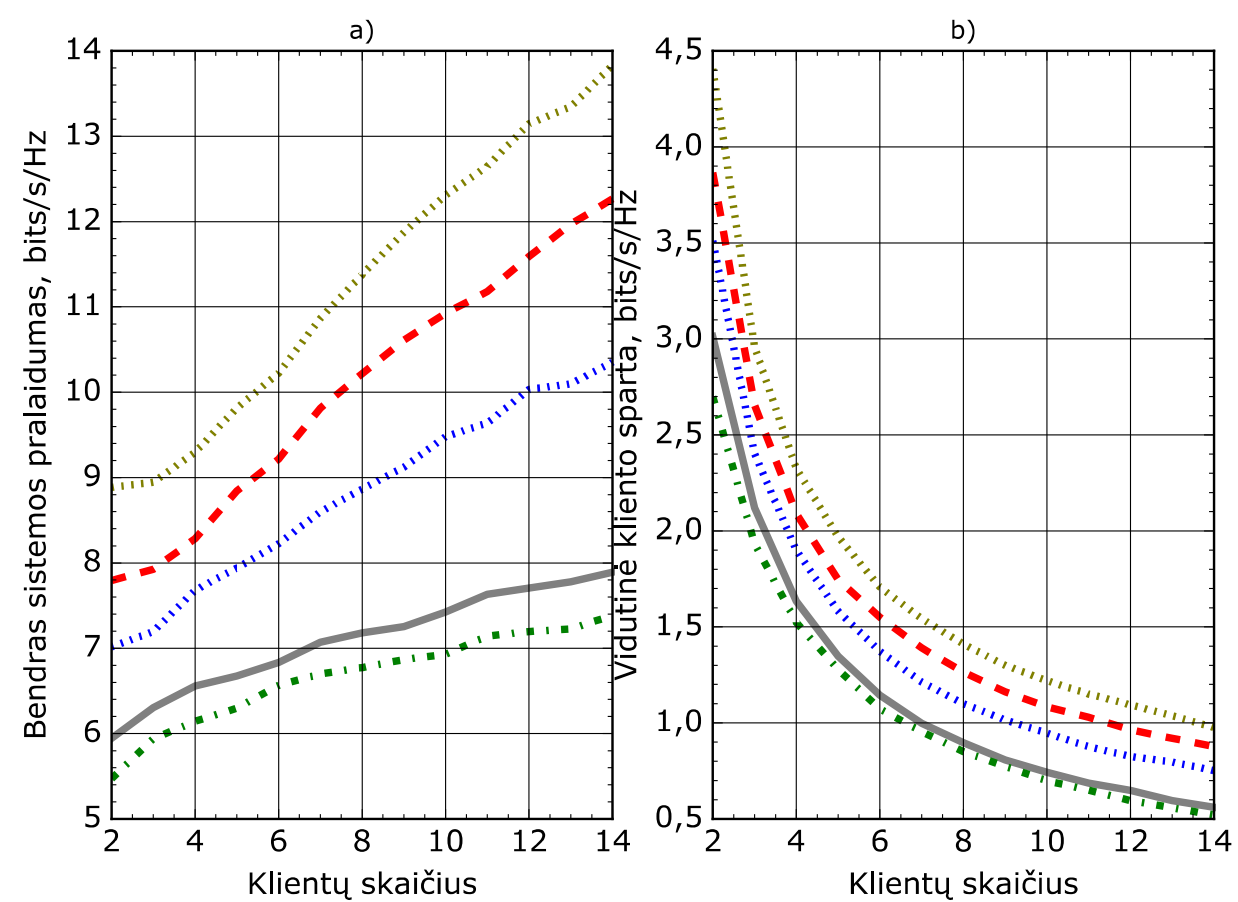

$$
\begin{array}{llll|}
\square & P_{\max }=0.1 \mathrm{~W} & =- & P_{\max }=2 \mathrm{~W} \\
\because \cdots & P_{\max }=0.2 \mathrm{~W} & \ldots \ldots & P_{\max }=4 \mathrm{~W} \\
\cdots \cdots & P_{\max }=1 \mathrm{~W} & & \\
\hline
\end{array}
$$

S2.4 pav. Bendras sistemos pralaidumas, kai naudojamas žaidimų teorija pagrịstas galios valdymo modelis veikiantis $100 \times 100 \mathrm{~m}$ plote a) Bendras sistemos pralaidumas b) Vidutinis vieno vartotojo pralaidumas

Modeliavimai atliekami remiantis (ITU-R Recommendation P.1238 2001), kai triukšmo lygis $n_{0}=10^{-12}$, o siųstuvo galios kaina $c_{i}=1$. Modeliavimo metu, vartotojų skaičius kito nuo 2 iki 14, o bendras visų vartotoju pralaidumas išreikštas kaip bendras perdavimo sistemos našumas. Siųstuvai buvo išdèlioti $100 \times 100 \mathrm{~m}$ patalpose atsitiktiniu būdu. Siekiant užtikrinti modeliavimo patikimumą, buvo atlikti 5000 modeliavimo iteracijų. Ivertinus modeliavimo rezultatus, nustatyta, kad didesné siųstuvo galia leidžia pasiekti didesni sistemos pralaidumą. Kaip papildoma priemonè ịvardijama dinaminè imtuvo jautrumo lygio kontrolè. Kiekvienas Wi-Fi ịrenginys, turi galimybę valdyti imtuvo jautrumą, tokiu būdu galima apriboti jo girdimumo zoną ir taip sumažinti triukšmo ịtaką. Nustačius dinamines ribas, kai imtuvo jautrumas sumažinamas, atsiranda galimybè naudoti didesnę siųstuvų perdavimo galią, kuri užtikrintų didesnę skvarbą ir tinklo aprèptį. 


\section{Eksperimentinis keliụ dažnių bevielès sistemos tyrimas}

Siekiant įvertinti Multi-Poll tinklo galimybes buvo įdiegta grupe įrenginių atviroje erdvëje. Šių testų tikslas ịvertinti, kaip MP sugeba aptarnauti didelị įrenginių skaičių. Testavimo metu naudota iki 83 įrenginių. Bazinès stotys sujungtos pagal pateiktą S3.1 paveiksle, 23 klientai yra instaliuoti $5 \mathrm{~km}$ atstumu, 60 klientų yra instaliuota $200 \mathrm{~m}$ atstumu nuo bazinių stočių.

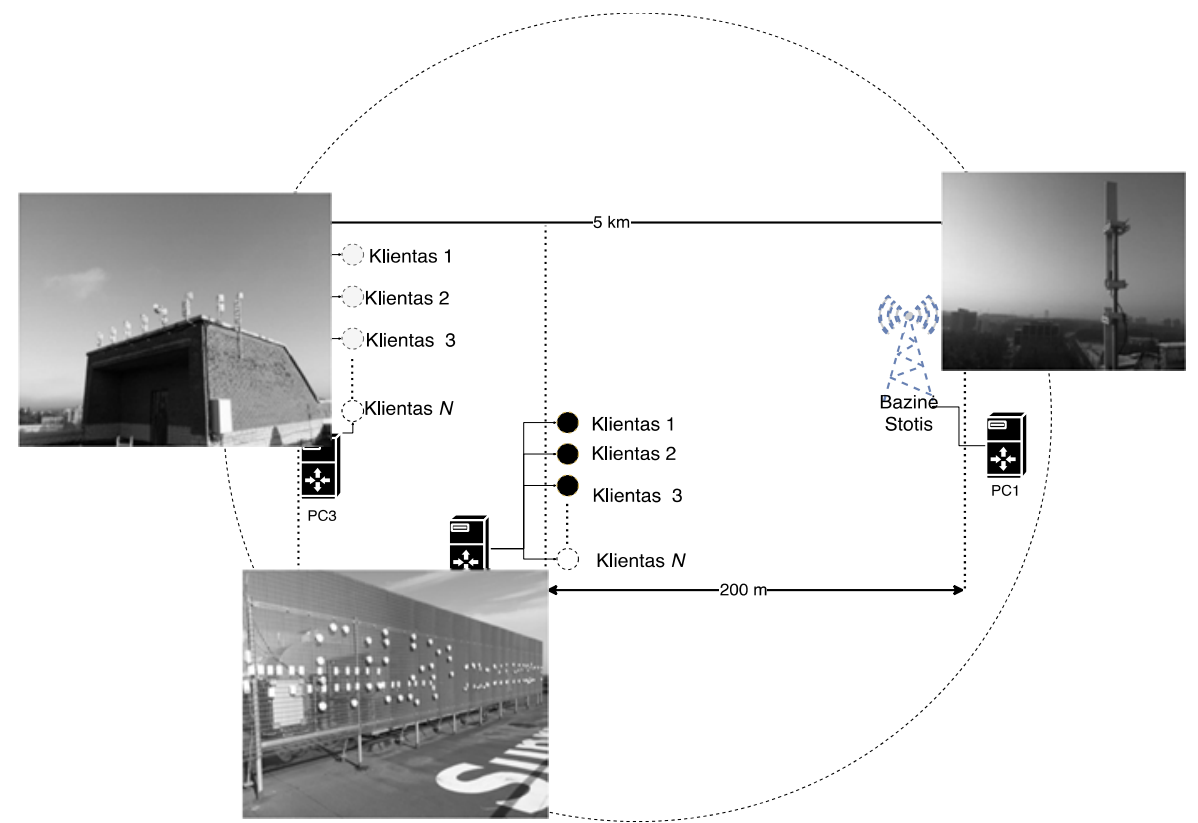

S3.1 pav. Tinklo įrenginio išdèstymo topologija

Sudarytas įrenginių scenarijus, kuris buvo pritaikytas testuoti tiek paslëptos stoties scenarijui, tiek IEEE 802.11 EDCA, PCF ir Multi-Poll protokolų scenarijams. Papildomai buvo atliekami ir siųstuvo perdavimo galios bandymai, kai tame pačiame arba gretimame dažnyje veikia keli sektoriaus tipo ịrenginiai. İrenginiai yra paremti Atheros lustų rinkinių ir naudoja $18 \mathrm{dBi}$ sektorinę anteną. Priklausomai nuo atstumo naudojami įrenginiai: Atheros AR9342 su 15 dBi kryptine antena arba 18 dBi kryptine antena. Maksimali siųstuvo galia siekia iki $29 \mathrm{dBm}$. Pasirinktas testavimo dažnis yra $5 \mathrm{GHz}$ diapazone, $5430 \mathrm{MHz}$. Kanalo plotis $40 \mathrm{MHz}$, signalų lygių ribos nuo $-45 \mathrm{dBm}$ iki $-55 \mathrm{dBm}$ įrenginiams, kurie prijungti $200 \mathrm{~m}$ atstumu ir nuo $-60 \mathrm{iki}-80 \mathrm{dBm}$ įrenginiams prijungtiems $5 \mathrm{~km}$ atstumu. Toks scenarijus leidžia tinkamai įvertinti Multi-Poll ir kitų protokolų dinamines charakteristikas esant skirtingoms moduliacijoms bei kolizijų padarinius. Pirmo testo metu siekta ìvertinti bendrą sistemos pralaidumo pokyti esant 13 aktyvių klientų skaičiui. S3.2 paveiksle matyti, kad esant tiktai 1 aktyviam klientui, MP atveju duomenų perdavimo sparta yra iki $57 \%$ didesnè lyginant su PCF standartu, kai yra 1 aktyvus klientas. Tuo 
tarpu kai visi 13 klientų tampa aktyvūs siuntèjai, pagerèjimas siekia iki $12 \%$ lyginant su PCF ir iki $79 \%$ lyginant su EDCA.

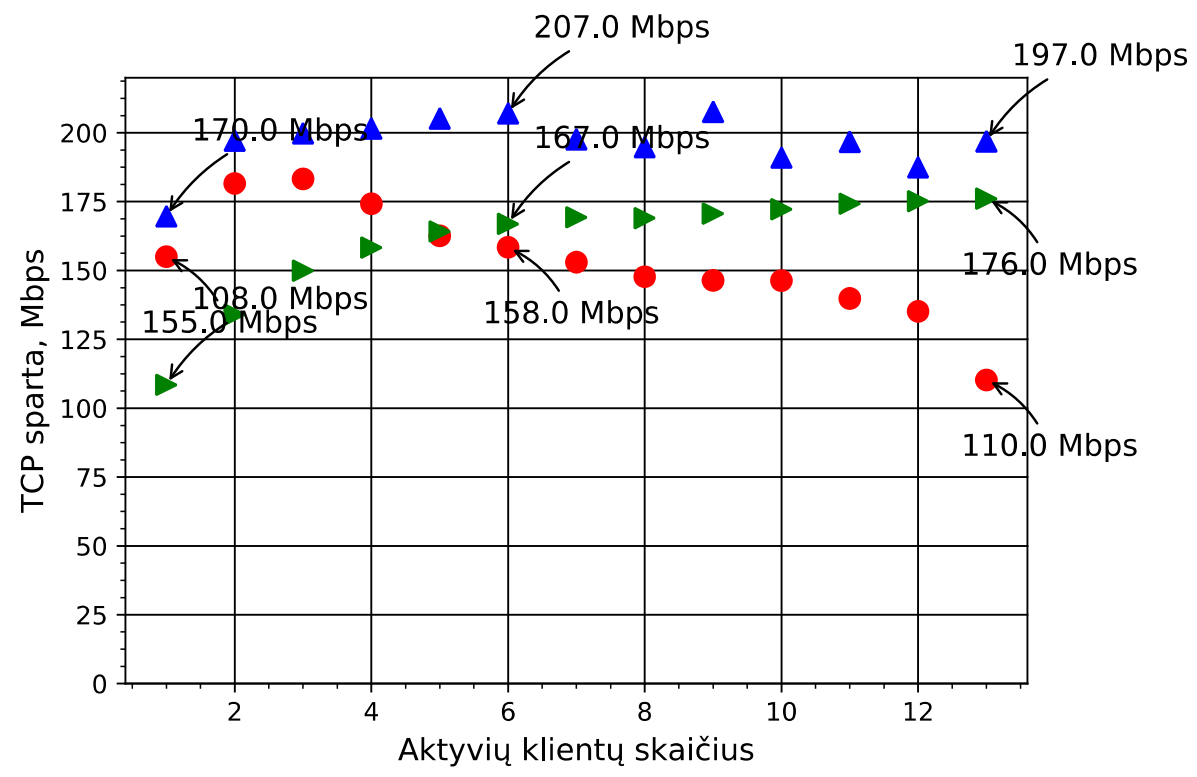

IEEE 802.11 EDCA protokolas

Siūlomas MP protokolas

IEEE 802.11 PCF (be varžymosi periodo)

S3.2 pav. Aktyvių ir neaktyvių klientų spartos paskirstymas esant kintančiam aktyvių klientų skaičiui

Kito testo metu buvo didinamas prisijungusių klientų skaičius iki 60, S3.3 paveikslas. Didejjant aktyvių klientų skaičiui, MP protokolas veikia našiau už PCF (be varžymosi periodo) ir EDCA protokolus. Tai galima paaiškinti tuo, kad PCF (be varžymosi periodo) netgi neesant duomenų siunčia papildomas užklausas likusiems neaktyviems klientams - vyksta aktyvi klientų kontrolè prie eterio prieigos. Todèl, esant 1 aktyviam klientui MP atveju gaunama duomenų perdavimo sparta siekia iki $182 \mathrm{Mbps,}$ EDCA iki $174 \mathrm{Mbps,} \mathrm{o} \mathrm{PCF} \mathrm{(be} \mathrm{varžymosi} \mathrm{lango)} \mathrm{atveju} \mathrm{iki} 10 \mathrm{Mbps}$. Atitinkamai, duomenų perdavimo pralaidumas padidejo iki $4 \%$ lyginant su EDCA perduodamų duomenų sparta.

Kito bandymo metu buvo vertinamas sistemos našumas esant iki 30 aktyvių klientų. Šiuo atveju, paimtas tarpinis variantas, kai dalis klientų buvo aktyviai siunčiantis, kita dalis nesiuntė jokių duomenų. Esant 30 aktyvių klientų, MP protokolas pasiekia iki $203 \mathrm{Mbps}$ perdavimo pralaidumą, tuo tarpu EDCA ir PCF atitinkamai pasiekia, $43 \mathrm{Mbps}$ ir iki $107 \mathrm{Mbps}$ perdavimo pralaidumą. Atlikus matavimą, nustatyta, kad MP atveju duomenų perdavimo pralaidumas padidèja iki 388 \% lyginant su EDCA kai yra 60 aktyviai siunčiančių klientų ir iki 50 \% lyginant su IEEE 802.11 PCF. 


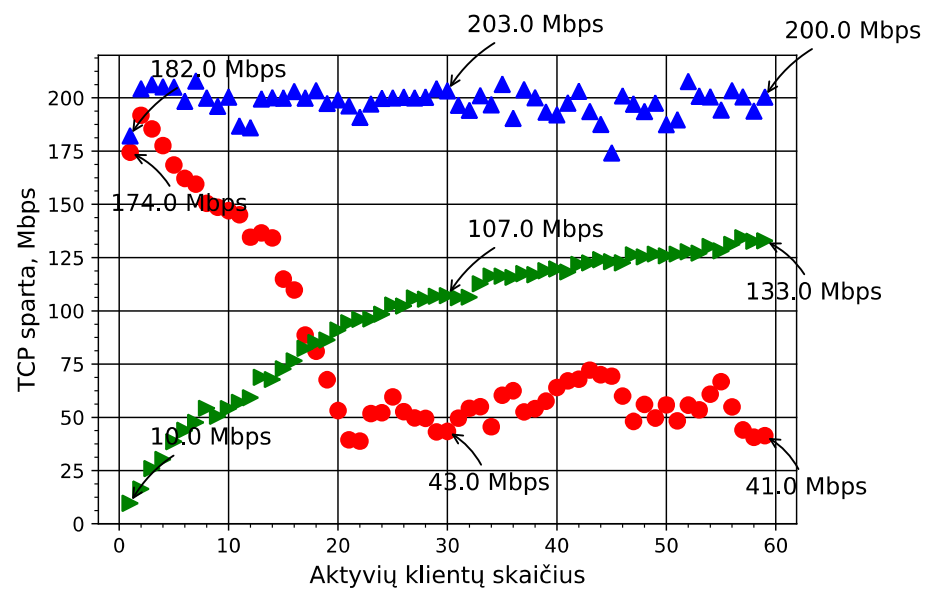

- IEEE 802.11 EDCA protokolas

\ Siūlomas MP protokolas

IEEE 802.11 PCF (be varžymosi periodo)

S3.3 pav. Sistemos pralaidumas esant 60 aktyvių klientų

Siekiant ịvertinti $5 \mathrm{GHz}$ ir $60 \mathrm{GHz}$ sistemos perdavimo spartą, buvo pasirinkta sistema sudaryta iš 3 dažnių posistemių $2.4 \mathrm{GHz}, 5 \mathrm{GHz}$ ir $60 \mathrm{GHz}$. IEEE 802.11ad standarte nurodoma FST (angl. Fast Session Transfer) funkcionalumas, kuris lemia greitą klientų persijungimą tarp skirtingų dažnio diapazonų ịvykus ryšio sutrikimams.

a) Bendras $60 \mathrm{GHz}$ sistemos pralaidumas be aktyvaus klientu perkélimo

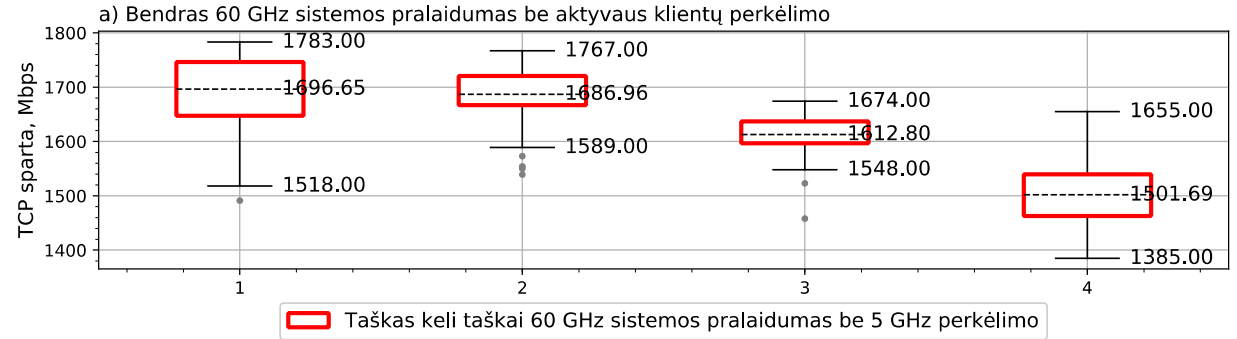

b) Bendras $60 \mathrm{GHz}$ sistemos pralaidumas naudojant klientu perkèlimą tarp $60 \mathrm{GHz}$ ir $5 \mathrm{GHz}$

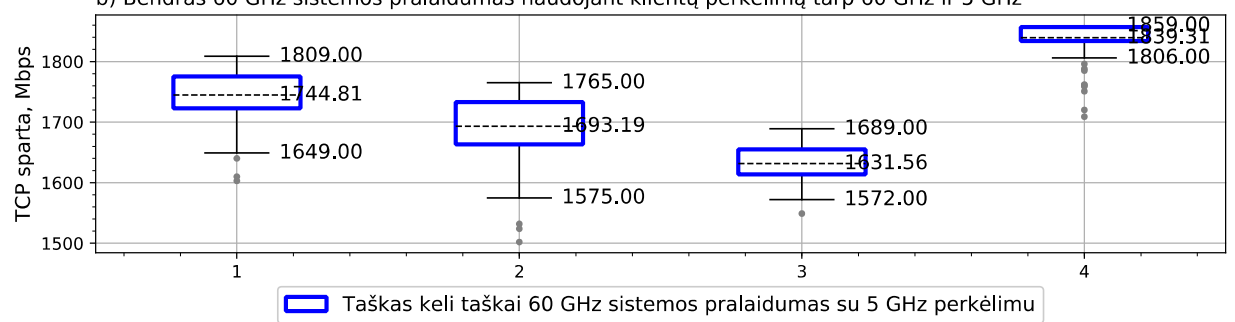

S3.4 pav. Sistemos pralaidumas kai naudojamas aktyvus klientų perkèlimas tarp $60 \mathrm{GHz}$ ir $5 \mathrm{GHz}$ 


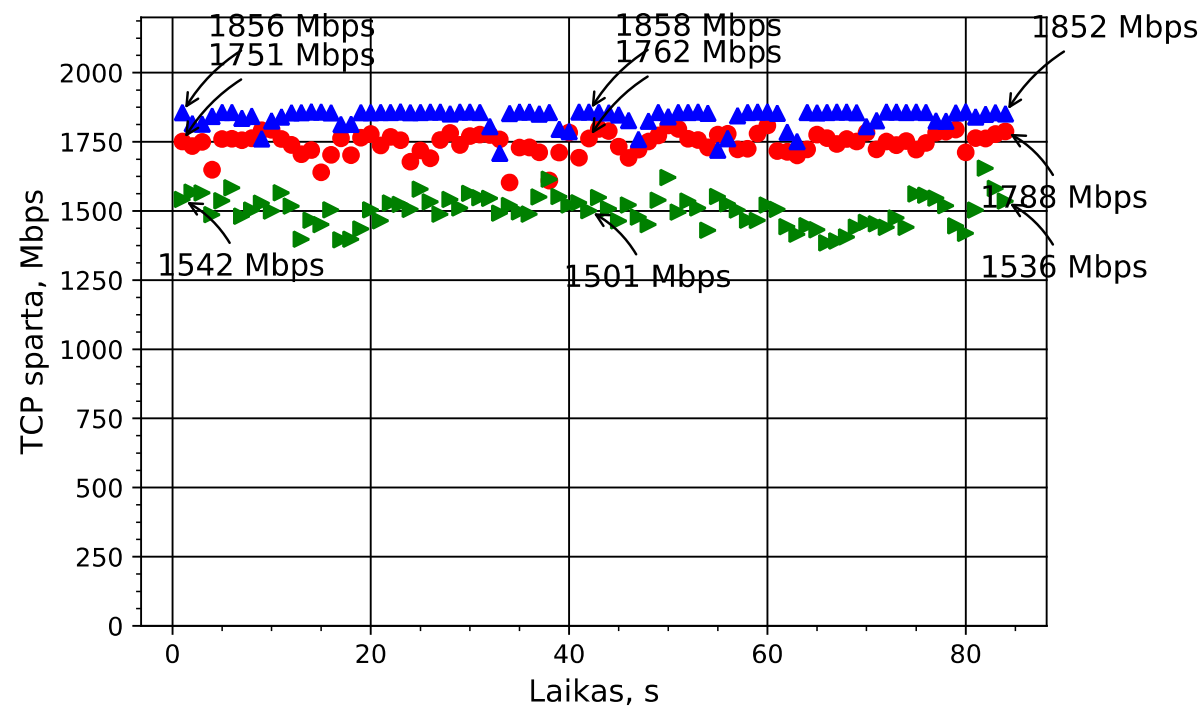

- Taškas-taškas $60 \mathrm{GHz}$ sistemos pralaidumas

- Taškas keli taškai $60 \mathrm{GHz}$ sistemos pralaidumas

\ Taškas keli taškai, kai naudojamas aktyvus klientu perkèlimas tarp $60 \mathrm{GHz}$ ir $5 \mathrm{GHz}$

S3.5 pav. IEEE 802.11ad perdavimo sparta, kai naudojama kelių dažnių sistema

Tačiau naujai pasiūlytas FST algoritmas leistų efektyviau išnaudoti radijo kanalo resursus, kai aktyvūs ir neaktyvūs klientai, būtų nukreipiami tarp $5 \mathrm{GHz}$ ir $60 \mathrm{GHz}$ diapazonų. Tai leistų efektyviau išnaudoti radijo kanalo resursus bei spręsti ryšio sutrikimo problemas, taškas keli taškai tinklo topologijose sudarytose iš $5 \mathrm{GHz}$ ir $60 \mathrm{GHz}$ posistemių.

S3.4 ir S3.5 paveiksle vaizduojama taškas keli taškai matavimo rezultatai, šiuo atveju lyginamas duomenu perdavimo sparta, kai visi klientai dirba tiktai $60 \mathrm{GHz}$ diapazone ir kai vienas iš klientų su žema perdavimo sparta perkeliamas į $5 \mathrm{GHz}$ diapazoną. Kaip ir IEEE 802.11 EDCA, klientai su blogu signalu ịtakoja bendrą sistemos pralaidumą. Todèl pasinaudojus pagerinus FST, galima padidinti bendrą sistemos spartą.

Siekiant ịvertinti šios sistemos našumą, pirmiausiai įvertinamas sistemos pralaidumas, kai yra tiktai vienas aktyvus klientas. Sudarius taškas-taškas sujungimo topologiją buvo išmatuota iki $1696 \mathrm{Mbps}$ TCP duomenų perdavimo sparta. Matavimai atlikti naudojant Nepim srauto generatorių, kuris siunte 1500 baitų paketus nustatytą laiką. Kito matavimo scenarijaus atveju, buvo prijungti 4 klientai ir sudaryta taškas keli taškai tinklo topologija. Matavimo metu nustatytas bendras sistemos pralaidumas siekiantis iki $1501 \mathrm{Mbps}$ TCP. Matavimo metu vienas iš klientų dèl apribotos siųstuvo galios turi žemą duomenų perdavimo spartą. Perkèlus ši klientą naudojant pasiūlytą aktyvų FST sprendimą i $5 \mathrm{GHz}$ dažnių juostą, bendras sistemos pralaidumas siekia iki 1839 Mbps. Tokiu būdu naudojant $5 \mathrm{GHz}$ ir $60 \mathrm{GHz}$ dažnių sistemą bei dinamiškai migruojant klientus pagal jų kokybinius signalų parametrus galima pasiekti iki $18 \%$ didesnį sistemos pralaidumą. 


\section{Bendrosios išvados}

1. Teorinių tyrimų metu išanalizuoti kanalo prieigos rèžimai. Nustatyta, kad IEEE 802.11n EDCA sprendimas, gali užtikrinti pakankamą duomenų perdavimo spartos, kai yra iki 10 aktyvių klientų skaičius, augant aktyvių klientų skaičiui perduodama duomenų sparta nebeužtikrinama.

2. Teorinių tyrimų metu įvertinta ir TDMA sistemos įtaka dabar egzistuojantiems EDCA tinklo įrenginiams, jos metu nustatyta, kad NAV parametras, nèra tinkamai apibrèžtas IEEE 802.11 standarte, tai leidžia TDMA sistemai sutrikdyti ịprastų ịrenginių veikimą tame pačiame ryšio kanale.

3. Sukurtas naujas MP IEEE 802.11 MAC lygmens protokolas padeda efektyviau kontroliuoti kanalo prieigą. Tyrimo metu nustatyta, kad šis protokolas yra efektyvus ir tinkamas naudoti taškas keli taškai sujungimuose lyginant su IEEE 802.11 EDCA ir IEEE 802.11 PCF (be varžymosi periodo).

4. Eksperimentinių tyrimų metu nustatyta, kad MP protokolas iki $79 \%$ padidina duomenų perdavimo spartą lyginant su EDCA ir iki $12 \%$ lyginant su PCF (be varžymosi periodo), kai yra 13 aktyvių klientų. Kai aktyvių klientų skaičius yra 60, MP protokolas pasiekia iki $50 \%$ didesnę duomenų perdavimo spartą lyginant su PCF (be varžymosi lango) ir iki 388 \% didesnę duomenų perdavimo spartą lyginant su EDCA protokolu.

5. Siekiant ịvertinti, kaip siųstuvo perdavimo galia įtakoja duomenų perdavimo spartą buvo atliekami siųstuvo galios bandymai. Nustatyta, jog ịrenginiai naudojantys žaidimų teorijos galios valdymą gali pasiekti iki $52 \%$ didesnę duomenų perdavimo spartą, kai įrenginiai dirba tame pačiame kanale ir iki $7 \%$ didesnę perdavimo spartą kai dirbama gretimuose kanaluose. Integracija su MP protokolu leistų dar efektyviau išnaudoti turimus radijo kanalo resursus.

6. Siekiant nustatyti $5 \mathrm{GHz}$ ir $60 \mathrm{GHz}$ radijo sistemų sąveiką buvo išbandytas naujas FST pakeitimas, kuris atlieka aktyvių ir neaktyvių klientų skirstymą tarp $5 \mathrm{GHz}$ ir $60 \mathrm{GHz}$ dažnio juostų. Pasiūlius ši algoritmą, galima pasiekti iki $18 \%$ didesnị sistemos pralaidumą taškas keli taškai tinkle topologijoje, kai egzistuoja klientas su prasta signalo kokybe.

7. Praktinių tyrimų metu nustatyta MP ir IEEE 802.11 EDCA protokolų sąveika, kai naudojamas bendras duomenų perdavimo kanalas. MP ir EDCA protokolai yra suderinami dirbti tame pačiame radijo kanale. 



\section{Annexes $^{1}$}

Annex A. Declaration of academic integrity

Annex B. The coauthors agreements to present publications for the dissertation defense

Annex C. Copies of scientific publications by the author on the topic of the dissertation

${ }^{1}$ The annexes are supplied in the enclosed compact disc 
Tomas CUZANAUSKAS

INVESTIGATION OF MEDIA ACCESS

CONTROL IN WIRELESS NETWORKS

Doctoral Dissertation

Technological Sciences,

Electrical and Electronic Engineering (01T)

Tomas CUZANAUSKAS

BELAIDŽIO RYŠIO TINKLỤ TERPĖS

PRIEIGOS VALDYMO TYRIMAS

Daktaro disertacija

Technologijos mokslai,

Elektros ir elektronikos inžinerija (01T)

201807 13. 14,5 sp. I. Tiražas 20 egz.

Vilniaus Gedimino technikos universiteto

leidykla "Technika“,

Saulètekio al. 11, 10223 Vilnius,

http://leidykla.vgtu./t

Spausdino UAB "Baltijos kopija“,

Kareivių g. 13B, 09109 Vilnius 